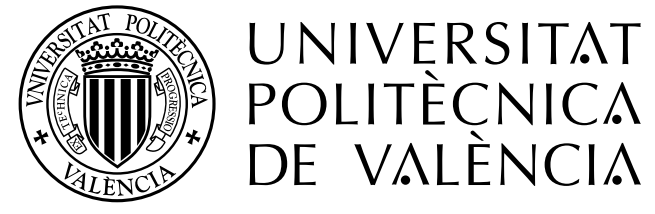

Ph.D. disseration

\title{
Design of Acoustic Lenses For ULTRASOUND FOCUSING APPLICATIONS
}

November 2021

\author{
Sergio Pérez-López \\ Centro de Tecnologías Físicas \\ Universitat Politècnica de València
}

\section{Supervisors:}

Dr. José Miguel Fuster

Dr. Pilar Candelas 



\section{Acknowledgements}

From these lines, I would like to thank everyone who helped me during this journey that started four years ago, in 2018. First of all, I deeply appreciate the support of my supervisors Dr. Pilar Candelas and Dr. José Miguel Fuster. Without their time, help, and guidance, this thesis would not have been possible. Thanks to all my research colleagues at the Centro de Tecnologías Físicas of the Universitat Politècnica de València. To Dr. Constanza Rubio, Prof. Antonio Uris, and Dr. Sergio Castiñeira-lbáñez, for the interesting scientific discussions and their continuous support. To my team colleague and friend Dr. Daniel Tarrazó-Serrano, with whom I spent countless hours in the laboratory and the office discussing all sorts of things about science and life, and who helped me a lot during my first years at the group. I also appreciate the support and help of Dr. Francisco Belmar, who introduced me to the group back in 2017, when I started working with a collaboration grant while I was finishing my Bachelor's degree.

I would also like to thank everyone at the microscopy team of the MultiScale Functional \& Molecular Imaging Laboratory at ETH Zürich for their warm welcome during my research stay. Especially, thanks to Prof. Daniel Razansky and Dr. Héctor Estrada for making the stay possible and for their supervision, and to Urs Hofmann for all his help and interesting scientific discussions.

Gracias también a mis amigos por todos los buenos momentos compartidos, ya que sin su compañía no habría podido llegar hasta aquí. Especialmente, gracias a Raúl, Sebas, Junior, Luis, Paco, Lydia y Víctor; y a Jen y Saul, que aunque ahora vivís en Noruega, sé que siempre puedo contar con vosotros. Y por último, a mis padres Paco y Lucía, y a mi hermano Alex, ya que sin su apoyo nada de esto habría sido posible. Gracias por vuestra infinita paciencia y vuestro apoyo constante. Os quiero. 



\section{Abstract}

Ultrasound focusing has many applications in a wide range of fields. Focused ultrasound is one of the main tools used by doctors all over the world to obtain biomedical images of different kind of tissues non-invasively. In the past decades, high intensity focused ultrasound (HIFU) appeared as one of the fundamental techniques for cancer treatment through non-invasive thermal tumor ablation. In addition, focused ultrasonic waves are recently emerging as one of the main tools to treat brain diseases, with novel disruptive techniques such as blood-brain barrier opening or neuromodulation. In industrial environments, ultrasonic waves are widely employed as one of the primary methods for the non-destructive evaluation (NDE) of materials and structures, as acoustic waves are able to penetrate deep into objects otherwise opaque using optical techniques. In this sense, designing structures capable of focusing ultrasonic waves is of great interest and relevance for the scientific, the industrial, and the biomedical sectors.

This thesis devises new designs of acoustic lenses capable of controlling the main parameters of the focused ultrasound beam, achieving different kinds of focusing profiles suitable for a wide variety of scenarios. In particular, Fresnel Zone Plates (FZPs), commonly used in optics, are designed and adapted to the ultrasound domain. A novel spatio-temporal modulation technique capable of controlling the ultrasound focus location in both time and space is presented, increasing the versatility of this kind of devices. New design techniques based on applying a binary sequence to FZPs are also demonstrated, such as Cantor fractal sequences or generalized M-bonacci sequences, which modify the focusing properties of the lens, including the number, location, and shape of the different acoustic foci. In addition, acoustic jets generated by liquid-filled spherical lenses are devised for near-field high resolution imaging, demonstrating their applicability in the ultrasound domain. It is demonstrated that, by changing the inner liquid of the spherical lens or by tuning the mixing ratio between two liquids, the main focal parameters of the ultrasonic jet can be accurately controlled. The proposed designs are validated using both numerical simulations and experimental measure- 
ments, paving the way for the use of these kind of structures in focused ultrasound applications. 


\section{Resumen}

La focalización de ultrasonidos tiene muchas aplicaciones en una gran variedad de áreas tanto científicas como industriales. Los ultrasonidos focalizados son una de las herramientas principales usada por médicos en todo el mundo para obtener imágenes biomédicas de diferentes tipos de tejidos y órganos de manera no invasiva. En las últimas décadas, el uso de ultrasonidos focalizados de alta intensidad (HIFU, por sus siglas en inglés) ha surgido como una de las técnicas principales para el tratamiento de cáncer mediante la ablación térmica de tumores de manera no invasiva. Además, los ultrasonidos focalizados están emergiendo en los últimos años como uno de los métodos más prometedores para el tratamiento de las enfermedades cerebrales, con la aparición de nuevas técnicas disruptivas como la apertura reversible de la barrera hematoencefálica o la neuromodulación. En entornos industriales, los ultrasonidos son ampliamente utilizados como uno de los métodos principales para la evaluación no destructiva de materiales y estructuras, debido a que las ondas acústicas pueden penetrar en los objetos a distancias donde la luz no puede debido a la elevada absorción y dispersión. En este sentido, diseñar estructuras capaces de focalizar ultrasonidos es de una gran relevancia tanto para la comunidad científica como para los sectores médicos e industriales.

Esta tesis presenta nuevos diseños de lentes acústicas capaces de controlar los parámetros principales del haz de ultrasonidos focalizados, proporcionando diferentes tipos de perfiles de focalización adecuados para una gran variedad de aplicaciones y escenarios. En particular, se han diseñado y adaptado al campo de los ultrasonidos las lentes de Fresnel (Fresnel Zone Plates, FZPs), ampliamente utilizadas en el campo de la óptica. Se ha presentado una nueva técnica de modulación espacio-temporal capaz de controlar la posición del foco de ultrasonidos tanto en espacio como en tiempo, aumentando así la versatilidad de este tipo de dispositivos. También se ha demostrado el funcionamiento en el campo de la acústica de nuevos diseños basados en aplicar secuencias binarias a una lente de Fresnel convencional, como las secuencias fractales de Cantor o las secuencias de M-bonacci generalizadas, capaces de modificar las propiedades de focalización de 
las lentes, incluyendo el número, posición y forma de los focos acústicos. Además, se introduce un nuevo diseño de lentes esféricas rellenas de líquido capaces de generar jets ultrasónicos, con mucho potencial en aplicaciones de imagen de alta resolución en campo cercano. Se ha demostrado que, cambiando el líquido interno de la lente o ajustando el ratio de mezcla entre dos líquidos, se pueden controlar los parámetros principales del jet. Los diseños propuestos en la tesis han sido validados tanto empleando simulaciones numéricas como realizando medidas experimentales, allanando el camino para el uso de este tipo de estructuras en aplicaciones de focalización de ultrasonidos. 


\section{Resum}

La focalització d'ultrasons té moltes aplicacions en moltes àrees científiques i industrials. Els ultrasons focalitzats són una de les eines principals utilitzada per metges a tot el món per obtenir imatges biomèdiques de diferents tipus de teixits i òrgans de manera no invasiva. En les últimes dècades, els ultrasons focalitzats d'alta intensitat (HIFU, per les seues sigles en anglès) han aparegut com una de les tècniques principals per al tractament de càncer mitjançant l'ablació de tumors de manera no invasiva. A més, els ultrasons focalitzats estan emergint en els últims anys com un dels mètodes més prometedors per al tractament de les malalties cerebrals, amb l'aparició de noves tècniques disruptives com l'obertura reversible de la barrera hematoencefàlica o la neuromodulació. En entorns industrials, els ultrasons són àmpliament utilitzats com un dels mètodes principals per a l'avaluació no destructiva de materials $\mathrm{i}$ estructures, pel fet que les ones acústiques poden penetrar en els objectes a distàncies on la llum no pot a causa de l'elevada absorció i dispersió. En aquest sentit, dissenyar estructures capaces de focalitzar ultrasons és d'una gran rellevància tant per a la comunitat científica com per als sectors mèdics $i$ industrials.

Aquesta tesi presenta nous dissenys de lents acústiques capaços de controlar els paràmetres principals del feix d'ultrasons focalitzats, proporcionant diferents tipus de perfils de focalització adequats per a una gran varietat d'aplicacions i escenaris. En particular, s'han dissenyat $\mathrm{i}$ adaptat al camp dels ultrasons les lents de Fresnel (Fresnel Zone Plates, FZPs), àmpliament utilitzades en el camp de l'òptica. S'ha presentat una nova tècnica de modulació espai-temporal capaç de controlar la posició del focus d'ultrasons tant en espai com en temps, augmentant així la versatilitat d'aquest tipus de dispositius. També s'ha demostrat el funcionament en el camp de l'acústica de nous dissenys basats en aplicar seqüències binàries a una lent de Fresnel convencional, com les seqüències fractals de Cantor o les seqüències de M-bonacci generalitzades, capaces de modificar les propietats de focalització de les lents, incloent el nombre, posició i forma dels focus acústics. A més, s'introdueix un nou disseny de lents esfèriques plenes de líquid capaces de 
generar jets ultrasònics, amb molt potencial en aplicacions d'imatge d'alta resolució en camp proper. S'ha demostrat que, canviant el líquid intern de la lent o ajustant la ràtio de barreja entre dos líquids, es poden controlar els paràmetres principals del jet. Els dissenys proposats en la tesi han estat validats tant emprant simulacions numèriques com realitzant mesures experimentals, aplanant el camí per a l'ús d'aquest tipus d'estructures en aplicacions de focalització d'ultrasons. 


\section{Contents}

1 Introduction $\quad 1$

1.1 Focused Ultrasound Applications ................ 2

1.2 Acoustic Lenses . . . . . . . . . . . . . . . . . . . . . . 4

1.3 Motivation and Objectives .............. . . 7

1.4 Structure of the Thesis . . . . . . . . . . . . . . . 8

References ........................... . . 8

2 Fundamentals of Acoustic Wave Diffraction and Propagation $\quad 19$

2.1 Diffraction . . . . . . . . . . . . . . . . . . . . 19

2.2 Propagation ......................... 23

2.3 Pressure Radiation from a Circular Planar Transducer . . . . . . . 24

2.4 Numerical Simulations . . . . . . . . . . . . . . . . . . . 26

2.5 Experimental Measurements . . . . . . . . . . . . . . . 27

References ........................ . . . 29

3 On the use of Phase Correction Rings on Fresnel Zone Plates with $\begin{array}{ll}\text { Ultrasound Piston Emitters } & 31\end{array}$

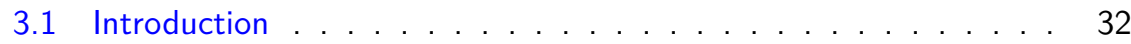


3.2 Results . . . . . . . . . . . . . . . . . . 32

3.3 Conclusions ......................... . . 42

References . . . . . . . . . . . . . . . . . . 42

4 Bifocal Ultrasound Focusing using Bi-Fresnel Zone Plate Lenses 45

4.1 Introduction . . . . . . . . . . . . . . . . . 46

4.2 BiFZP Lens Design . . . . . . . . . . . . . . . . . . . 47

4.3 Experimental Results and Discussion . . . . . . . . . . . . 52

4.4 Conclusions ........................ 54

References .......................... . . . 55

5 Transient Analysis of Fresnel Zone Plates for Ultrasound Focusing Applications $\quad 59$

5.1 Introduction ........................ 60

5.2 Results .......................... 61

5.2.1 FZP Transient Duration . . . . . . . . . . . . . . . 61

5.2.2 FZP Transient Response . . . . . . . . . . . . . . 62

5.3 Methods ........................ 69

5.3.1 Rayleigh-Sommerfeld Spectrum . . . . . . . . . . . . 69

5.3.2 Numerical Model . . . . . . . . . . . . . . . . 70

5.4 Conclusion . . . . . . . . . . . . . . . . . . 70

References ....................... . . 71

6 Spatio-temporal Ultrasound Beam Modulation to Sequentially achieve $\begin{array}{ll}\text { Multiple Foci with a Single Planar Monofocal Lens } & 73\end{array}$

6.1 Introduction .......................... 74 
6.2 Results . . . . . . . . . . . . . . . . . . . . 75

6.3 Discussion ........................ . . . 81

6.4 Materials and Methods . . . . . . . . . . . . . . . . 82

6.4 .1 Experimental Set-up . . . . . . . . . . . . . . . 82

6.4.2 Axial Spectrum Simulation .............. . 83

References ....................... . . 83

Supplementary Information . . . . . . . . . . . . . . . . . 86

7 Fractal Lenses based on Cantor Binary Sequences for Ultrasound Focusing Applications $\quad 93$

7.1 Introduction . . . . . . . . . . . . . . . . . . . . . 94

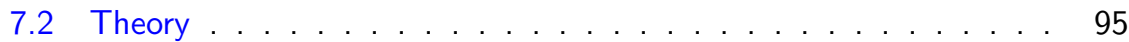

7.3 Experimental Results . . . . . . . . . . . . . . . . . . 100

7.4 Conclusion . . . . . . . . . . . . . . . . . . 104

References . . . . . . . . . . . . . . . . . . 104

8 M-bonacci Zone Plates for Ultrasound Focusing 107

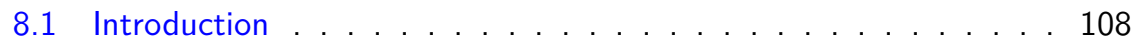

8.2 M-bonacci Zone Plates . . . . . . . . . . . . . . . . . . . 109

8.2.1 Design and Focusing Properties . . . . . . . . . . . . 109

8.2.2 $\gamma$-Parameter .................... 115

8.3 Experimental Results and Discussion . . . . . . . . . . . . . . . 119

8.4 Conclusion . . . . . . . . . . . . . . . . . . . . . . 121

References .......................... . . . 121 
9 Liquid-liquid Core-shell Configurable Mesoscale Spherical Acoustic Lens with Subwavelength Focusing

9.1 Introduction . . . . . . . . . . . . . . . . 126

9.2 Results . . . . . . . . . . . . . . . . . . . . 127

9.3 Discussion . . . . . . . . . . . . . . . . . . . . 134

References . . . . . . . . . . . . . . . . . . 135

10 Tunable Subwavelength Ultrasound Focusing in Mesoscale Spherical

$\begin{array}{lr}\text { Lenses using Liquid Mixtures } & 139\end{array}$

10.1 Introduction . . . . . . . . . . . . . . . . . . 140

10.2 Results . . . . . . . . . . . . . . . . . . . 142

10.3 Discussion . . . . . . . . . . . . . . . . . . 147

10.4 Methods . . . . . . . . . . . . . . . . . . . 148

10.4.1 Numerical Model . . . . . . . . . . . . . . . . . . 148

10.4.2 Experimental Set-up. . . . . . . . . . . . . 149

References . . . . . . . . . . . . . . . . . . . 149

11 Conclusions $\quad 153$

11.1 Summary . . . . . . . . . . . . . . . . . . . 153

11.2 Main Conclusions . . . . . . . . . . . . . . . . . 156 


\section{Chapter 1}

\section{Introduction}

The term ultrasound refers to acoustic waves propagating in matter, either in solid, liquid or gaseous state. In this sense, acoustics can be defined as the the science of sound [1], including its generation, propagation, and reception, where sound does not only refer to phenomena in the audible regime, but also in lower (infrasound) and higher (ultrasound) frequencies. A wave, on the other hand, is the vibrational movement (at a finite speed) of a disturbance from one point to another in matter [2]. This vibrational movement represents a transmission of mechanical energy through matter.

The audible regime of an average person ranges from $20 \mathrm{~Hz}$ to $20 \mathrm{kHz}$ [3], and therefore, ultrasound includes the study of acoustic waves at frequencies higher than $20 \mathrm{kHz}$. Depending on how the disturbance moves respect to the propagation direction, two kinds of acoustic waves can be distinguished. If the disturbance moves along the propagation direction, the acoustic wave is longitudinal, whereas if the disturbance moves perpendicular to the propagation direction, the acoustic wave is transverse. Ultrasound has many and diverse applications in a large amount of scientific and professional areas. Next section covers, in particular, the applications of focused ultrasound, as it is the key application scenario of the work developed in this thesis. 


\subsection{Focused Ultrasound Applications}

Focused ultrasound (FUS) is a term employed to denote ultrasonic waves that direct the mechanical energy, and therefore the amplitude of the vibrational displacement, into a single or a specific set of spatial points, known as focus or foci. FUS has a huge range of applications in many areas, mainly due to its non-ionizing mechanical nature and its cost effective performance. Ultrasonic waves can penetrate deep into objects or tissues where optical waves cannot due to absorption and scattering. Moreover, ultrasound transducers are generally cheaper and easier to design compared to other imaging modalities such as MRI, X-Ray, or optical systems [4]. Nowadays, the two main FUS application scenarios are the biomedical and the industrial fields.

Mainly, industrial applications of FUS are based on non-destructive testing (NDT) of materials and structures to detect flaws or deformations [5-10]. In addition, high-power FUS is also employed for industrial cleaning, welding, and cutting of materials [11]. Other interesting industrial application of FUS is sonochemistry. Sonochemistry uses bubble cavitation produced by the ultrasonic waves in liquids to control and activate certain chemical reactions [12, 13]. Ultrasound has applications in the food industry too. For instance, low-power ultrasound is currently used to monitor the composition and physicochemical properties of food, while high-power ultrasound is employed in various steps of food processing chains $[14,15]$.

Nowadays, the main and most widely extended application of FUS in medicine is biomedical imaging of soft tissues [4]. This kind of conventional pulseecho techniques allow to measure the sizes and distances of the different organs, which can be extremely useful to diagnose certain kind of pathologies. Moreover, ultrasonic imaging has been used since decades ago for pregnancy monitoring and assessment of the development of the baby.

One of the most growing FUS medical applications is high intensity focused ultrasound (HIFU). HIFU can be used to treat different types of tumors using high intensity ultrasonic waves directed towards the targeted cancerous tissues, which results in thermal ablation of the cancerous cells [16-18]. One important advancement in this field is the combination of HIFU with magnetic resonance imaging (MRI) techniques $[19,20]$, which allows to control the temperature of the targeted cancerous tissue and its surroundings in real time, therefore enhancing the efficacy of the treatment and reducing the ablation of healthy tissues.

In the past years, novel plane wave illumination techniques allowed for real-time ultrasound imaging at thousands of frames per second [21, 22]. Plane 
wave imaging uses plane waves to simultaneously illuminate all the tissue, instead of performing beam steering to scan each angle of the tissue as conventional phased-array configurations do. Here, it is worth noting that the focusing process is not performed in transmission nor in reception of the acoustic wavefront, but focusing is actually achieved applying signal processing methods to the received ultrasonic signals. This new technique paved the way for a wide range of new biomedical applications. For instance, performing ultrasonic imaging at thousands of frames per second allowed researchers to perform doppler imaging of small blood vessels and to monitor blood flow in different tissues [23-25]. Ultrafast ultrasound imaging has also had a great impact on neuroscience with the appearance of new functional ultrasound techniques (fUS) $[26,27]$, which are used to visualize and track hemodynamic changes in brain vasculature. This allows to track neural activation and behaviour patterns, which is a key aspect necessary to reach a better understanding of how the brain works. Another key advantage of imaging ultrasonic waves at thousands of frames per second is that the system is able to analyze acoustic waves with very slow speeds of sounds, of approximately $1-10$ $\mathrm{m} / \mathrm{s}$. Interestingly, the shear (or transverse) speed of sound of soft biological tissues is in that order of magnitude, which has allowed the appearance of a new ultrasonic imaging modality: shear wave elastography [28-31]. This technique is able to estimate the elastic parameters of tissues (i.e. Young's modulus and shear modulus), which provides an indication of the stiffness of the tissue and can be very helpful in the diagnosis of tumors and malformations. However, it is important to keep in mind that this kind of fUS techniques do not employ acoustic lenses and they always rely on phased-arrays to perform plane wave imaging, so this technique is outside the application scope of this thesis.

One of the main obstacles to treat diseases affecting the central nervous system (CNS), is the blood-brain barrier (BBB), which is a thin membrane made of endothelial cells surrounding the blood vessels of the brain that prevents drugs from penetrating into the CNS. In this sense, in the past decades FUS emerged as one of the most powerful tools to safely and non-invasively open the BBB in specific target areas [32-34]. Using microbubbles injected directly into the blood stream, FUS can make the microbubbles vibrate and temporarily open the junctions of the BBB, therefore allowing drugs to penetrate the CNS and treat a wide range of brain diseases.

Another big application of FUS in neuroscience is ultrasound neuromodulation (UNM). FUS can deliver mechanical energy to neurons deep into the brain, and trigger neural activation in specific regions of the brain (those located inside the ultrasound focus) [35-37]. This allows scientist to expand their knowledge of the brain behaviour, non-invasively. 


\subsection{Acoustic Lenses}

FUS can be generated with either passive structures that transform unfocused acoustic energy into focused energy or with active transducer phased-arrays. The passive structures capable of focusing the incident ultrasonic energy into one or various focal spots are named acoustic lenses, and their working principle is based on modifying the phase and/or amplitude of the incident field so that the wavefront leaving the lens converges constructively at the focus, and therefore the acoustic energy is maximized at that particular spatial point [38]. Alternatively, phased-arrays consist of a series of ultrasound transducers, which input excitation amplitude and phase can be individually controlled [39-41]. This allows to actively design an ultrasonic wavefront with the desired number and location of foci. The main advantage of phased-arrays compared to acoustic lenses is, therefore, that the generated wavefront, and thus the focusing pattern, can be actively reconfigured, which enables interesting applications such as acoustic tweezers and particle trapping and levitation [42-44]. In contrast, once and acoustic lens is manufactured, its focusing pattern is constrained and cannot be controlled with the same degree of freedom as that of a phased-array. However, despite this important constrain, employing acoustic lenses instead of phased-arrays is becoming more and more popular among scientist, due to two key advantages. First of all, they are orders of magnitude cheaper than phased-arrays, and considerably easier and faster to manufacture. Second, the advance in 3D printing and material science techniques allowed scientific teams to design and manufacture custom acoustic lenses within a few hours, or even minutes, using commercially available 3D printers. This fact, combined with the advance in ultrasound holography research, which enabled scientists to tailor complex 3D ultrasonic focal patterns, resulted in a big growth of interest in acoustic lenses in the past years. Therefore, acoustic lenses are becoming more and more appealing to researchers, as they provide a faster and simpler way to achieve acoustic focusing, with reduced size and cost compared to phased-arrays.

Classical acoustic lenses consist mainly of concave spherically-shaped passive structures directly coupled to the transducer, with a fixed single focal distance $[38,45,46]$, although focusing can also be achieved by shaping the piezoelectric material $[47,48]$. In the last decades, researchers have devised new ways to achieve acoustic focusing. From all these novel methods, two stand out as the most widely analyzed and investigated in the literature: acoustic metasurfaces and holograms.

The concept of metasurface was first introduced and demonstrated in the fields of optics and microwaves, where the option of designing materials and surfaces with negative magnetic permeability and electric permittivity attracted closed attention due to their multiple applications, including wave focusing [49- 
54]. Later, metasurfaces were also investigated and demonstrated in acoustics [55], where they have proven to be a very appealing option to design ultrasonic lenses. Acoustic metasurfaces are made of a group of unit cells, smaller in size than the operating wavelength. Each unit cell is designed to provide a specific amplitude and phase at its output plane, so that the combination of the different contributions of the unit cells generates an acoustic wavefront capable of achieving the desired focusing pattern. Several unit cell designs have been presented in recent years. Among all the possibilities, three stand out as the most widely employed unit cell design techniques: Helmholtz resonators, coiling-up space structures, and subwavelength slits. Acoustic metasurfaces made of Helmholtz resonators are based on tuning individual subwavelength Helmholtz resonators to provide the desired complex transmission coefficient of the unit cell [56-58]. Coiling-up unit cells employ subwavelength labyrinthine paths with variable lengths and widths, so that at the output of the labyrinth the acoustic wave has the desired phase [59-63]. The latter option consists of using an array of deep subwavelength slits, tuned to provide the desired pressure distribution at its output plane $[64,65]$.

Nowadays, the other main paradigm to design acoustic lenses is based on acoustic holograms. Acoustic holograms are single elements that produce a pressure field at its output plane (known as the holographic plane) capable of achieving complex 3D pressure patterns. The design method consists of backpropagating this $3 \mathrm{D}$ pressure target pattern to the holographic plane, and then obtaining a lens that can provide the complex conjugate of the backpropagated field. Obtaining the final lens design requires an iterative forward and backward propagation algorithm to achieve a lens height profile capable of generating the desired pressure distribution at the target plane. Early research was developed in the $90 \mathrm{~s}$, demonstrating that an holographic lens directly coupled to a single ultrasound transducer is capable of achieving high resolution focusing at the desired spatial locations $[66,67]$. In the past decade, with the fast advances in 3D printers and $3 \mathrm{D}$ printing technologies and materials, 3D printed holographic lenses appeared as a very promising alternative to achieve complex ultrasound focusing patterns [68]. Since then, 3D printed holograms have become one of the most versatile methods to achieve FUS, with very short manufacturing cost and time [69-76].

Despite their great potential and versatility, both metasurfaces and acoustic holograms have important drawbacks. On the one hand, acoustic metasurfaces need a complete 3D design and optimisation of each one of their unit cells, which can be difficult to achieve. Moreover, the manufacturing process of the subwavelength structures can be challenging for high frequency ultrasound. On the other hand, holographic lenses usually have complex geometries and height profiles that require high manufacturing precision, which can be limiting in some application scenarios. In addition, when the acoustic wavefront propagates through heterogeneous media, such as biomedical applications involving transcranial propagation, 
performing iterative 3D full-wave simulations can be challenging, resulting in very time-consuming simulations with heavy computational burden. However, this is not a specific drawback associated to this kind of devices, as whenever heterogeneities are present in the acoustic medium, full-wave simulations are required to properly characterize the acoustic focus and its aberrations.

In this regard, Fresnel Zone Plates (FZPs) are a very appealing alternative for researchers working with FUS. FZPs are a simpler yet powerful and versatile choice to achieve ultrasound focusing. This type of devices has been used since decades ago in many fields, such as optics [77-79], X-rays [80, 81], and microwaves [82, 83]. FZPs are planar monofocal lenses made of a series of concentric rings, with decreasing width. The position of each ring depends on the focusing parameters, such as the focal distance and the operating wavelength. The resolution of the lens can be controlled by adjusting the number of rings, and therefore the size of the lens. Moreover, the planar geometry of FZPs can be a big advantage over holographic lenses and acoustic metasurfaces, resulting in an easier and faster manufacturing process, although FZPs offer less flexibility in terms of focusing pattern configuration. Interestingly, in the past years, novel designs based on FZPs were introduced for optical applications. These designs are based on applying a binary sequence to the different rings of the lens. This binary sequence controls the activation or deactivation of each ring, and depending on the type of binary sequence, different focusing patterns are obtained. Two main types of binary sequences stand out for their interesting influence on the focusing profile: Fibonacci and Cantor sequences. Cantor fractal binary sequences provide focusing profiles with a main focus and multiple secondary foci, with interesting self-similarity properties $[84,85]$, while Fibonacci binary sequences applied on the FZP rings result in bifocal intensity patterns $[86,87]$.

In the ultrasound field, early research was carried out in the $70 \mathrm{~s}$, when researchers designed and manufactured FZPs by shaping the piezoelectric electrodes as FZP rings [88-90], demonstrating the use of this kind of devices in the ultrasound domain. Later, in the $80 \mathrm{~s}$ and $90 \mathrm{~s}$, researchers implemented FZPs by directly etching the piezoelectric material with the shape of an FZP, increasing the efficiency of this devices [91, 92]. In the past decade, new ultrasonic FZP designs were introduced, separating the lens from the transducer or directly coupling an interchangeable lens to the surface of the transducer, therefore increasing their versatility and application scenario [93]. Subsequently, novel designs based on FZPs were presented, such as lenses capable of creating an ultrasonic vortex $[94,95], 3 D$ printed FZPs $[96,97]$, and stretchable FZPs that provide a tunable focal spot [98].

In the optical domain, another interesting design technique was introduced in the last years, capable of generating optical jets by using dielectric spheres. 
These optical jets, which received the name of photonic nanojets, are generated at the near-field shadow side of the sphere when the sphere is illuminated by a plane wave, and its lateral resolution can overcome the diffraction limit [99-101]. The jet is generated by the scattered modes at the sphere, which results in evanescent waves that provide the lateral information required to achieve a focal spot narrower than $\lambda / 2$. Recently, in 2017 researchers I.V. Minin and O.V. Minin proposed an acoustic analogue to the photonic nanojet in the acoustic domain [102], and later in the same year they experimentally demonstrated with collaborators J.H. Lopes et al. the generation of an ultrasonic jet using a solid Rexolite sphere immersed in water $[103,104]$, achieving a lateral resolution of $\lambda / 2$ with a sphere radius of just $4.14 \lambda$. Further research proved that ultrasonic jets can exceed $\lambda / 2$ resolutions $[105,106]$.

\subsection{Motivation and Objectives}

As shown in the previous sections, FUS has many applications in the industrial and biomedical fields, and therefore devising new ways to achieve ultrasound focusing and improving current techniques is of great scientific significance. This thesis aims to further develop the use of FZPs in the ultrasound domain, extending their capabilities by using novel design techniques introduced in the optical domain based on binary sequences, and devising novel beam modulation methods capable of controlling the ultrasound focus. Moreover, as a result of a collaboration with Prof. Igor V. Minin and Prof. Oleg V. Minin from Tomsk Polytechnic University, the application of spherical liquid-filled containers to generate ultrasonic jets is also investigated. Ultrasonic jets can break the diffraction limit, and therefore can potentially become a very appealing alternative for near-field ultrasound imaging applications. Thus, by increasing the versatility of acoustic FZPs and ultrasonic jets, this thesis wants to expand the use and capabilities of this kind of devices, as they are simple structures that can provide good focusing performance.

The main objectives of this thesis can be listed as:

1. Design and adapt FZP lenses for the ultrasound domain.

2. Implement beam modulation techniques that allow for an accurate control of the ultrasound beam.

3. Design acoustic lenses based on binary sequences to increase the versatility and application scenario of conventional FZPs.

4. Investigate spherical lenses capable of generating ultrasonic jets with tunable focusing parameters. 


\subsection{Structure of the Thesis}

This thesis is arranged as a collection of published journal articles. A total of 8 journal papers are included, related to the different objectives established in the thesis. The dissertation is structured as follows:

- Chapter 1: state of the art review of ultrasonic lenses and the main applications of focused ultrasound. Main objectives and structure of this dissertation.

- Chapter 2: fundamental theory of acoustic wave diffraction and propagation. Summary of the employed numerical methods and their implementation, and overview of the experimental set-up and the measurement process.

- Chapters 3-10: collection of published journal papers. By topic, the chapters are arranged as follows:

- Chapters 3-6: design of FZP lenses in the ultrasound domain and beam modulation techniques. A method to compensate the distortion introduced by piston transducers in the focusing profiles of ultrasonic FZPs is devised. Bifocal focusing profiles are achieved by combining two conventional FZPs with different focal distances. The transient response of FZPs is analyzed, and a new beam modulation technique capable of controlling the ultrasound focus in space and time is presented. These chapters correspond to objectives 1 and 2 of this thesis.

- Chapters 7-8: application of binary sequences to modify the focusing properties of conventional FZPs. Cantor and M-bonacci binary sequences are analyzed and experimentally demonstrated in the ultrasound domain, modifying the focusing profiles of FZP lenses with bifocal intensity distributions and fractal multifocal profiles. These chapters are related to objective number 3 of this thesis.

- Chapters 9-10: investigation of spherical lenses to generate ultrasonic jets. New designs based on spherical liquid-filled lenses are presented, providing a way of achieving ultrasonic jets with tunable focal parameters. These chapters are related to objective 4 of this thesis.

- Chapter 11: discussion and final conclusions of this thesis.

\section{References}

[1] A. D. Pierce, Acoustics. Springer International Publishing, 3rd ed., 2019. 
[2] D. T. Blackstock, Fundamentals of Physical Acoustics. John Wiley \& Sons, Inc., 1st ed., 2000.

[3] L. E. Kinsler, A. R. Frey, A. B. Coppens, and J. V. Sanders, Fundamentals of Acoustics. John Wiley \& Sons, Inc., 4th ed., 2000.

[4] H. Azhari, Basics of Biomedical Ultrasound for Engineers. John Wiley \& Sons, Inc., 1st ed., feb 2010.

[5] L. W. Schmerr, Fundamentals of Ultrasonic Nondestructive Evaluation. Springer Series in Measurement Science and Technology, Springer International Publishing, 2nd ed., 2016.

[6] B. Lee and E. Furgason, "An Evaluation of Ultrasound NDE Correlation Flaw Detection Systems," IEEE Transactions on Sonics and Ultrasonics, vol. 29, pp. 359-369, nov 1982.

[7] J. Peters, "Non-Contact Inspection of Composites Using Air-Coupled Ultrasound," in AIP Conference Proceedings, vol. 657, pp. 973-980, AIP, 2003.

[8] S. A. Titov, R. G. Maev, and A. N. Bogachenkov, "Pulse-echo NDT of adhesively bonded joints in automotive assemblies," Ultrasonics, vol. 48, pp. 537-546, nov 2008.

[9] V. Janapati, F. Kopsaftopoulos, F. Li, S. J. Lee, and F.-K. Chang, "Damage detection sensitivity characterization of acousto-ultrasound-based structural health monitoring techniques," Structural Health Monitoring, vol. 15, pp. 143-161, mar 2016.

[10] D. O. Dolmatov, D. Tarrazó-Serrano, G. A. Filippov, I. V. Minin, O. V. Minin, and D. A. Sednev, "Application of Phase-Reversal Fresnel Zone Plates for Improving The Elevation Resolution in Ultrasonic Testing with Phased Arrays," Sensors, vol. 19, p. 5080, nov 2019.

[11] G. Harvey, A. Gachagan, and T. Mutasa, "Review of high-power ultrasoundindustrial applications and measurement methods," IEEE Transactions on Ultrasonics, Ferroelectrics, and Frequency Control, vol. 61, pp. 481-495, mar 2014.

[12] N. Pokhrel, P. K. Vabbina, and N. Pala, "Sonochemistry: Science and Engineering," Ultrasonics Sonochemistry, vol. 29, pp. 104-128, mar 2016.

[13] L. Vernès, M. Abert-Vian, M. El Maâtaoui, Y. Tao, I. Bornard, and F. Chemat, "Application of ultrasound for green extraction of proteins from spirulina. Mechanism, optimization, modeling, and industrial prospects," Ultrasonics Sonochemistry, vol. 54, pp. 48-60, jun 2019. 
[14] T. Awad, H. Moharram, O. Shaltout, D. Asker, and M. Youssef, "Applications of ultrasound in analysis, processing and quality control of food: A review," Food Research International, vol. 48, pp. 410-427, oct 2012.

[15] F. Chemat, N. Rombaut, A.-G. Sicaire, A. Meullemiestre, A.-S. FabianoTixier, and M. Abert-Vian, "Ultrasound assisted extraction of food and natural products. Mechanisms, techniques, combinations, protocols and applications. A review," Ultrasonics Sonochemistry, vol. 34, pp. 540-560, jan 2017.

[16] J. E. Kennedy, G. R. ter Haar, and D. Cranston, "High intensity focused ultrasound: surgery of the future?," The British Journal of Radiology, vol. 76, pp. 590-599, sep 2003.

[17] J. Kennedy, F. Wu, G. ter Haar, F. Gleeson, R. Phillips, M. Middleton, and D. Cranston, "High-intensity focused ultrasound for the treatment of liver tumours," Ultrasonics, vol. 42, pp. 931-935, apr 2004.

[18] E. Maloney and J. H. Hwang, "Emerging HIFU applications in cancer therapy," International Journal of Hyperthermia, vol. 31, pp. 302-309, apr 2015.

[19] Y.-s. Kim, B. Keserci, A. Partanen, H. Rhim, H. K. Lim, M. J. Park, and M. O. Köhler, "Volumetric MR-HIFU ablation of uterine fibroids: Role of treatment cell size in the improvement of energy efficiency," European Journal of Radiology, vol. 81, pp. 3652-3659, nov 2012.

[20] M. Huisman, M. K. Lam, L. W. Bartels, R. J. Nijenhuis, C. T. Moonen, F. M. Knuttel, H. M. Verkooijen, M. van Vulpen, and M. A. van den Bosch, "Feasibility of volumetric MRI-guided high intensity focused ultrasound (MRHIFU) for painful bone metastases," Journal of Therapeutic Ultrasound, vol. 2, no. 1, p. 16, 2014.

[21] L. Sandrin, S. Catheline, M. Tanter, X. Hennequin, and M. Fink, "TimeResolved Pulsed Elastography with Ultrafast Ultrasonic Imaging," Ultrasonic Imaging, vol. 21, pp. 259-272, oct 1999.

[22] M. Tanter and M. Fink, "Ultrafast imaging in biomedical ultrasound," IEEE Transactions on Ultrasonics, Ferroelectrics, and Frequency Control, vol. 61, pp. 102-119, jan 2014.

[23] J. Bercoff, G. Montaldo, T. Loupas, D. Savery, F. Mézière, M. Fink, and M. Tanter, "Ultrafast compound doppler imaging: providing full blood flow characterization," IEEE Transactions on Ultrasonics, Ferroelectrics and Frequency Control, vol. 58, pp. 134-147, jan 2011. 
[24] J. Provost, C. Papadacci, J. E. Arango, M. Imbault, M. Fink, J.-L. Gennisson, M. Tanter, and M. Pernot, "3D ultrafast ultrasound imaging in vivo," Physics in Medicine and Biology, vol. 59, pp. L1-L13, oct 2014.

[25] C. Demene, T. Deffieux, M. Pernot, B.-F. Osmanski, V. Biran, J.-L. Gennisson, L.-A. Sieu, A. Bergel, S. Franqui, J.-M. Correas, I. Cohen, O. Baud, and M. Tanter, "Spatiotemporal Clutter Filtering of Ultrafast Ultrasound Data Highly Increases Doppler and fUltrasound Sensitivity," IEEE Transactions on Medical Imaging, vol. 34, pp. 2271-2285, nov 2015.

[26] S. L. Norman, D. Maresca, V. N. Christopoulos, W. S. Griggs, C. Demene, M. Tanter, M. G. Shapiro, and R. A. Andersen, "Single-trial decoding of movement intentions using functional ultrasound neuroimaging," Neuron, vol. 109, pp. 1554-1566.e4, may 2021.

[27] E. Macé, G. Montaldo, I. Cohen, M. Baulac, M. Fink, and M. Tanter, "Functional ultrasound imaging of the brain," Nature Methods, vol. 8, pp. 662664, aug 2011.

[28] J. Bercoff, M. Tanter, and M. Fink, "Supersonic shear imaging: a new technique for soft tissue elasticity mapping," IEEE Transactions on Ultrasonics, Ferroelectrics and Frequency Control, vol. 51, pp. 396-409, apr 2004.

[29] G. Montaldo, M. Tanter, J. Bercoff, N. Benech, and M. Fink, "Coherent plane-wave compounding for very high frame rate ultrasonography and transient elastography," IEEE Transactions on Ultrasonics, Ferroelectrics and Frequency Control, vol. 56, pp. 489-506, mar 2009.

[30] J.-L. Gennisson, T. Deffieux, M. Fink, and M. Tanter, "Ultrasound elastography: Principles and techniques," Diagnostic and Interventional Imaging, vol. 94, pp. 487-495, may 2013.

[31] R. M. Sigrist, J. Liau, A. E. Kaffas, M. C. Chammas, and J. K. Willmann, "Ultrasound Elastography: Review of Techniques and Clinical Applications," Theranostics, vol. 7, no. 5, pp. 1303-1329, 2017.

[32] R. M. Jones, L. Deng, K. Leung, D. McMahon, M. A. O'Reilly, and K. Hynynen, "Three-dimensional transcranial microbubble imaging for guiding volumetric ultrasound-mediated blood-brain barrier opening," Theranostics, vol. 8, no. 11, pp. 2909-2926, 2018.

[33] T. Mainprize, N. Lipsman, Y. Huang, Y. Meng, A. Bethune, S. Ironside, C. Heyn, R. Alkins, M. Trudeau, A. Sahgal, J. Perry, and K. Hynynen, "Blood-Brain Barrier Opening in Primary Brain Tumors with Non-invasive MR-Guided Focused Ultrasound: A Clinical Safety and Feasibility Study," Scientific Reports, vol. 9, p. 321, dec 2019. 
[34] A. Abrahao, Y. Meng, M. Llinas, Y. Huang, C. Hamani, T. Mainprize, I. Aubert, C. Heyn, S. E. Black, K. Hynynen, N. Lipsman, and L. Zinman, "First-in-human trial of blood-brain barrier opening in amyotrophic lateral sclerosis using MR-guided focused ultrasound," Nature Communications, vol. 10, p. 4373, dec 2019.

[35] J. Blackmore, S. Shrivastava, J. Sallet, C. R. Butler, and R. O. Cleveland, "Ultrasound Neuromodulation: A Review of Results, Mechanisms and Safety," Ultrasound in Medicine \& Biology, vol. 45, pp. 1509-1536, jul 2019.

[36] C. Rabut, S. Yoo, R. C. Hurt, Z. Jin, H. Li, H. Guo, B. Ling, and M. G. Shapiro, "Ultrasound Technologies for Imaging and Modulating Neural Activity," Neuron, vol. 108, pp. 93-110, oct 2020.

[37] H. A. S. Kamimura, A. Conti, N. Toschi, and E. E. Konofagou, "Ultrasound Neuromodulation: Mechanisms and the Potential of Multimodal Stimulation for Neuronal Function Assessment," Frontiers in Physics, vol. 8, may 2020.

[38] T. Tarnoczy, "Sound focussing lenses and waveguides," Ultrasonics, vol. 3, pp. 115-127, jul 1965.

[39] E. Ebbini and C. Cain, "Multiple-focus ultrasound phased-array pattern synthesis: optimal driving-signal distributions for hyperthermia," IEEE Transactions on Ultrasonics, Ferroelectrics and Frequency Control, vol. 36, pp. 540548, sep 1989.

[40] A. Casper, Dalong Liu, and E. S. Ebbini, "Realtime Control of Multiple-focus Phased Array Heating Patterns Based on Noninvasive Ultrasound Thermography," IEEE Transactions on Biomedical Engineering, vol. 59, pp. 95-105, jan 2012.

[41] A. Ilovitsh, T. Ilovitsh, J. Foiret, D. N. Stephens, and K. W. Ferrara, "Simultaneous Axial Multifocal Imaging Using a Single Acoustical Transmission: A Practical Implementation," IEEE Transactions on Ultrasonics, Ferroelectrics, and Frequency Control, vol. 66, pp. 273-284, feb 2019.

[42] C. R. P. Courtney, C. E. M. Demore, H. Wu, A. Grinenko, P. D. Wilcox, S. Cochran, and B. W. Drinkwater, "Independent trapping and manipulation of microparticles using dexterous acoustic tweezers," Applied Physics Letters, vol. 104, p. 154103, apr 2014.

[43] A. Marzo, S. A. Seah, B. W. Drinkwater, D. R. Sahoo, B. Long, and S. Subramanian, "Holographic acoustic elements for manipulation of levitated objects," Nature Communications, vol. 6, p. 8661, dec 2015. 
[44] A. Marzo and B. W. Drinkwater, "Holographic acoustic tweezers," Proceedings of the National Academy of Sciences, vol. 116, pp. 84-89, jan 2019.

[45] J. Li, C. R. Friedrich, and R. S. Keynton, "Design and fabrication of a miniaturized, integrated, high-frequency acoustical lens-transducer system," Journal of Micromechanics and Microengineering, vol. 12, pp. 219-228, may 2002.

[46] J. Jang and J. Chang, "Design and Fabrication of Double-Focused Ultrasound Transducers to Achieve Tight Focusing," Sensors, vol. 16, p. 1248, aug 2016.

[47] G. Lockwood, D. Turnbull, and F. Foster, "Fabrication of high frequency spherically shaped ceramic transducers," IEEE Transactions on Ultrasonics, Ferroelectrics and Frequency Control, vol. 41, pp. 231-235, mar 1994.

[48] J. Cannata, T. Ritter, Wo-Hsing Chen, R. Silverman, and K. Shung, “Design of efficient, broadband single-element (20-80 MHz) ultrasonic transducers for medical imaging applications," IEEE Transactions on Ultrasonics, Ferroelectrics and Frequency Control, vol. 50, pp. 1548-1557, nov 2003.

[49] V. G. Veselago, "THE ELECTRODYNAMICS OF SUBSTANCES WITH SIMULTANEOUSLY NEGATIVE VALUES OF $\varepsilon$ AND $\mu$," Soviet Physics Uspekhi, vol. 10, pp. 509-514, apr 1968.

[50] N. Yu, P. Genevet, M. A. Kats, F. Aieta, J.-P. Tetienne, F. Capasso, and Z. Gaburro, "Light Propagation with Phase Discontinuities: Generalized Laws of Reflection and Refraction," Science, vol. 334, pp. 333-337, oct 2011.

[51] S. Maci, G. Minatti, M. Casaletti, and M. Bosiljevac, "Metasurfing: Addressing Waves on Impenetrable Metasurfaces," IEEE Antennas and Wireless Propagation Letters, vol. 10, pp. 1499-1502, 2011.

[52] C. L. Holloway, E. F. Kuester, J. A. Gordon, J. O'Hara, J. Booth, and D. R. Smith, "An Overview of the Theory and Applications of Metasurfaces: The Two-Dimensional Equivalents of Metamaterials," IEEE Antennas and Propagation Magazine, vol. 54, pp. 10-35, apr 2012.

[53] M. Khorasaninejad, F. Aieta, P. Kanhaiya, M. A. Kats, P. Genevet, D. Rousso, and F. Capasso, "Achromatic Metasurface Lens at Telecommunication Wavelengths," Nano Letters, vol. 15, pp. 5358-5362, aug 2015.

[54] A. A. High, R. C. Devlin, A. Dibos, M. Polking, D. S. Wild, J. Perczel, N. P. de Leon, M. D. Lukin, and H. Park, "Visible-frequency hyperbolic metasurface," Nature, vol. 522, pp. 192-196, jun 2015. 
[55] B. Assouar, B. Liang, Y. Wu, Y. Li, J.-C. Cheng, and Y. Jing, "Acoustic metasurfaces," Nature Reviews Materials, vol. 3, pp. 460-472, dec 2018.

[56] J. Lan, Y. Li, Y. Xu, and X. Liu, "Manipulation of acoustic wavefront by gradient metasurface based on Helmholtz Resonators," Scientific Reports, vol. 7, p. 10587, dec 2017.

[57] S. Qi and B. Assouar, "Ultrathin acoustic metasurfaces for reflective wave focusing," Journal of Applied Physics, vol. 123, p. 234501, jun 2018.

[58] J.-p. Xia, X.-t. Zhang, H.-x. Sun, S.-q. Yuan, J. Qian, and Y. Ge, “Broadband Tunable Acoustic Asymmetric Focusing Lens from Dual-Layer Metasurfaces," Physical Review Applied, vol. 10, p. 014016, jul 2018.

[59] Y. Li, B. Liang, X. Tao, X.-f. Zhu, X.-y. Zou, and J.-c. Cheng, "Acoustic focusing by coiling up space," Applied Physics Letters, vol. 101, p. 233508, dec 2012.

[60] Y. Xie, W. Wang, H. Chen, A. Konneker, B.-I. Popa, and S. A. Cummer, "Wavefront modulation and subwavelength diffractive acoustics with an acoustic metasurface," Nature Communications, vol. 5, p. 5553, dec 2014.

[61] Y. Li, G. Yu, B. Liang, X. Zou, G. Li, S. Cheng, and J. Cheng, "Threedimensional Ultrathin Planar Lenses by Acoustic Metamaterials," Scientific Reports, vol. 4, p. 6830, may 2015.

[62] J. Qian, J.-p. Xia, H.-x. Sun, S.-q. Yuan, Y. Ge, and X.-z. Yu, "Broadband acoustic focusing by cavity structures with phase manipulations," Journal of Applied Physics, vol. 122, p. 244501, dec 2017.

[63] D.-C. Chen, X.-F. Zhu, Q. Wei, D.-J. Wu, and X.-J. Liu, "Broadband acoustic focusing by Airy-like beams based on acoustic metasurfaces," Journal of Applied Physics, vol. 123, p. 044503, jan 2018.

[64] J. Chen, J. Xiao, D. Lisevych, A. Shakouri, and Z. Fan, “Deepsubwavelength control of acoustic waves in an ultra-compact metasurface lens," Nature Communications, vol. 9, p. 4920, dec 2018.

[65] J. Chen, J. Rao, D. Lisevych, and Z. Fan, "Broadband ultrasonic focusing in water with an ultra-compact metasurface lens," Applied Physics Letters, vol. 114, p. 104101, mar 2019.

[66] R. Lalonde, A. Worthington, and J. Hunt, "Field conjugate acoustic lenses for ultrasound hyperthermia," IEEE Transactions on Ultrasonics, Ferroelectrics and Frequency Control, vol. 40, pp. 592-602, sep 1993. 
[67] R. Lalonde and J. Hunt, "Variable frequency field conjugate lenses for ultrasound hyperthermia," IEEE Transactions on Ultrasonics, Ferroelectrics and Frequency Control, vol. 42, pp. 825-831, sep 1995.

[68] K. Melde, A. G. Mark, T. Qiu, and P. Fischer, "Holograms for acoustics," Nature, vol. 537, pp. 518-522, sep 2016.

[69] M. D. Brown, T. J. Allen, B. T. Cox, and B. E. Treeby, "Control of optically generated ultrasound fields using binary amplitude holograms," in 2014 IEEE International Ultrasonics Symposium, pp. 1037-1040, IEEE, sep 2014.

[70] M. D. Brown, B. T. Cox, and B. E. Treeby, "Design of multi-frequency acoustic kinoforms," Applied Physics Letters, vol. 111, p. 244101, dec 2017.

[71] S. Jiménez-Gambín, N. Jiménez, J. M. Benlloch, and F. Camarena, "Holograms to Focus Arbitrary Ultrasonic Fields through the Skull," Physical Review Applied, vol. 12, p. 014016, jul 2019.

[72] M. D. Brown, "Phase and amplitude modulation with acoustic holograms," Applied Physics Letters, vol. 115, p. 053701, jul 2019.

[73] S. Jiménez-Gambín, N. Jiménez, and F. Camarena, "Transcranial Focusing of Ultrasonic Vortices by Acoustic Holograms," Physical Review Applied, vol. 14, p. 054070, nov 2020.

[74] Z. Ma, A. W. Holle, K. Melde, T. Qiu, K. Poeppel, V. M. Kadiri, and P. Fischer, "Acoustic Holographic Cell Patterning in a Biocompatible Hydrogel," Advanced Materials, vol. 32, p. 1904181, jan 2020.

[75] Z. Ma, K. Melde, A. G. Athanassiadis, M. Schau, H. Richter, T. Qiu, and P. Fischer, "Spatial ultrasound modulation by digitally controlling microbubble arrays," Nature Communications, vol. 11, p. 4537, dec 2020.

[76] M. D. Brown, B. T. Cox, and B. E. Treeby, "Stackable acoustic holograms," Applied Physics Letters, vol. 116, p. 261901, jun 2020.

[77] M. Young, "Zone Plates and Their Aberrations," Journal of the Optical Society of America, vol. 62, p. 972, aug 1972.

[78] R. S. Rodrigues Ribeiro, P. Dahal, A. Guerreiro, P. A. S. Jorge, and J. Viegas, "Fabrication of Fresnel plates on optical fibres by FIB milling for optical trapping, manipulation and detection of single cells," Scientific Reports, vol. 7, p. 4485, dec 2017.

[79] H. Kim, J. Kim, H. An, Y. Lee, G.-y. Lee, J. Na, K. Park, S. Lee, S.-Y. Lee, B. Lee, and Y. Jeong, "Metallic Fresnel zone plate implemented on an optical fiber facet for super-variable focusing of light," Optics Express, vol. 25, p. 30290, nov 2017. 
[80] J. Kirz, "Phase zone plates for x rays and the extreme uv," Journal of the Optical Society of America, vol. 64, p. 301, mar 1974.

[81] W. Yashiro, Y. Takeda, A. Takeuchi, Y. Suzuki, and A. Momose, "Hard-XRay Phase-Difference Microscopy Using a Fresnel Zone Plate and a Transmission Grating," Physical Review Letters, vol. 103, p. 180801, oct 2009.

[82] H. Hristov and M. Herben, "Millimeter-wave Fresnel-zone plate lens and antenna," IEEE Transactions on Microwave Theory and Techniques, vol. 43, no. 12 , pp. 2779-2785, 1995.

[83] H. D. Hristov and J. M. Rodriguez, "Design Equation for Multidielectric Fresnel Zone Plate Lens," IEEE Microwave and Wireless Components Letters, vol. 22, pp. 574-576, nov 2012.

[84] G. Saavedra, W. D. Furlan, and J. A. Monsoriu, "Fractal zone plates," Optics Letters, vol. 28, p. 971, jun 2003.

[85] O. Mendoza-Yero, M. Fernández-Alonso, G. Mínguez-Vega, J. Lancis, V. Climent, and J. A. Monsoriu, "Fractal generalized zone plates," Journal of the Optical Society of America A, vol. 26, p. 1161, may 2009.

[86] J. A. Monsoriu, A. Calatayud, L. Remon, W. D. Furlan, G. Saavedra, and P. Andres, "Bifocal Fibonacci Diffractive Lenses," IEEE Photonics Journal, vol. 5, pp. 3400106-3400106, jun 2013.

[87] F. Machado, V. Ferrando, W. D. Furlan, and J. A. Monsoriu, "Diffractive m-bonacci lenses," Optics Express, vol. 25, p. 8267, apr 2017.

[88] G. Chao, B. Auld, and D. Winslow, "Focusing and Scanning of Acoustic Beams with Fresnel Zone Plates," in 1972 Ultrasonics Symposium, pp. 140143, IEEE, 1972.

[89] S. A. Farnow and B. A. Auld, "Acoustic Fresnel zone plate transducers," Applied Physics Letters, vol. 25, pp. 681-682, dec 1974.

[90] S. A. Farnow and B. A. Auld, "An Acoustic Phase Plate Imaging Device," in Acoustical Holography, pp. 259-273, Boston, MA: Springer US, 1975.

[91] K. Yamada and H. Shimizu, "Planar-Structure Focusing Lens for Acoustic Microscope," in IEEE 1985 Ultrasonics Symposium, pp. 755-758, IEEE, 1985.

[92] B. Hadimioglu, E. Rawson, R. Lujan, M. Lim, J. Zesch, B. Khuri-Yakub, and C. Quate, "High-efficiency Fresnel acoustic lenses," in 1993 Proceedings IEEE Ultrasonics Symposium, pp. 579-582 vol.1, IEEE, 1993. 
[93] D. C. Calvo, A. L. Thangawng, M. Nicholas, and C. N. Layman, "Thin Fresnel zone plate lenses for focusing underwater sound," Applied Physics Letters, vol. 107, p. 014103, jul 2015.

[94] N. Jiménez, V. Romero-García, L. M. García-Raffi, F. Camarena, and K. Staliunas, "Sharp acoustic vortex focusing by Fresnel-spiral zone plates," Applied Physics Letters, vol. 112, p. 204101, may 2018.

[95] R. D. Muelas-Hurtado, J. L. Ealo, and K. Volke-Sepúlveda, “Active-spiral Fresnel zone plate with tunable focal length for airborne generation of focused acoustic vortices," Applied Physics Letters, vol. 116, p. 114101, mar 2020 .

[96] R. Lirette and J. Mobley, "Focal zone characteristics of stepped Fresnel and axicon acoustic lenses," in Proceedings of Meetings on Acoustics, vol. 31, p. 045001, 2017.

[97] D. Tarrazó-Serrano, S. Pérez-López, P. Candelas, A. Uris, and C. Rubio, "Acoustic Focusing Enhancement In Fresnel Zone Plate Lenses," Scientific Reports, vol. 9, p. 7067, dec 2019.

[98] X. Xia, Y. Li, F. Cai, H. Zhou, T. Ma, and H. Zheng, "Ultrasonic tunable focusing by a stretchable phase-reversal Fresnel zone plate," Applied Physics Letters, vol. 117, p. 021904, jul 2020.

[99] P. Ferrand, J. Wenger, A. Devilez, M. Pianta, B. Stout, N. Bonod, E. Popov, and H. Rigneault, "Direct imaging of photonic nanojets," Optics Express, vol. 16, p. 6930 , may 2008

[100] A. Heifetz, S.-C. Kong, A. V. Sahakian, A. Taflove, and V. Backman, "Photonic Nanojets," Journal of Computational and Theoretical Nanoscience, vol. 6, pp. 1979-1992, sep 2009.

[101] B. S. Luk'yanchuk, R. Paniagua-Domínguez, I. Minin, O. Minin, and Z. Wang, "Refractive index less than two: photonic nanojets yesterday, today and tomorrow [Invited]," Optical Materials Express, vol. 7, p. 1820, jun 2017.

[102] O. V. Minin and I. V. Minin, "Acoustojet: acoustic analogue of photonic jet phenomenon based on penetrable 3D particle," Optical and Quantum Electronics, vol. 49, p. 54, feb 2017.

[103] J. H. Lopes, M. A. B. Andrade, J. P. Leão-Neto, J. C. Adamowski, I. V. Minin, and G. T. Silva, "Focusing Acoustic Beams with a Ball-Shaped Lens beyond the Diffraction Limit," Physical Review Applied, vol. 8, p. 024013, aug 2017. 
[104] J. H. Lopes, M. A. B. Andrade, J. C. Adamowski, J. P. Leao-Neto, and G. T. Silva, "Focusing beyond the diffraction limit with acoustic jets," in 2017 IEEE International Ultrasonics Symposium (IUS), pp. 1-4, IEEE, sep 2017.

[105] T. Kekkonen, J. Makinen, J. Hyvonen, A. Kuronen, T. Kotiaho, A. Salmi, and E. Haggstrom, "3D acoustic jet," in 2019 IEEE International Ultrasonics Symposium (IUS), pp. 586-589, IEEE, oct 2019.

[106] D. Veira Canle, T. Kekkonen, J. Mäkinen, T. Puranen, H. J. Nieminen, A. Kuronen, S. Franssila, T. Kotiaho, A. Salmi, and E. Hæggström, "Practical realization of a sub- $\lambda / 2$ acoustic jet," Scientific Reports, vol. 9, p. 5189, dec 2019. 


\section{Chapter 2}

\section{Fundamentals of Acoustic Wave Diffraction and Propagation}

The main objective of this chapter is to offer an introduction to the fundamental theory and equations of acoustic wave diffraction and propagation, as well as the main numerical methods employed in this thesis to compute the simulations presented in next chapters. In addition, the experimental set-up and measurement process is also described.

\subsection{Diffraction}

When a pressure wavefront encounters an abrupt edge or corner, as happens when an incident acoustic field reaches the different rings of an FZP lens, the wavefront is diffracted, generating an interference pattern. Given a pressure field at a point $\boldsymbol{r}^{\prime}=\left(x^{\prime}, y^{\prime}, 0\right)$ incident on an aperture located at $z=0, p_{0}\left(\boldsymbol{r}^{\prime}\right)$, the diffracted pressure at the point $\boldsymbol{r}=(x, y, z)$ can be calculated by using the Rayleigh-Sommerfeld integral [1, 2],

$$
p(\boldsymbol{r})=\iint_{S} p_{0}\left(\boldsymbol{r}^{\prime}\right) t\left(\boldsymbol{r}^{\prime}\right) \frac{e^{j k\left\|\boldsymbol{r}-\boldsymbol{r}^{\prime}\right\|}}{2 \pi\left\|\boldsymbol{r}-\boldsymbol{r}^{\prime}\right\|}\left(\frac{1}{\left\|\boldsymbol{r}-\boldsymbol{r}^{\prime}\right\|}-j k\right) \frac{z}{\left\|\boldsymbol{r}-\boldsymbol{r}^{\prime}\right\|} d S,
$$




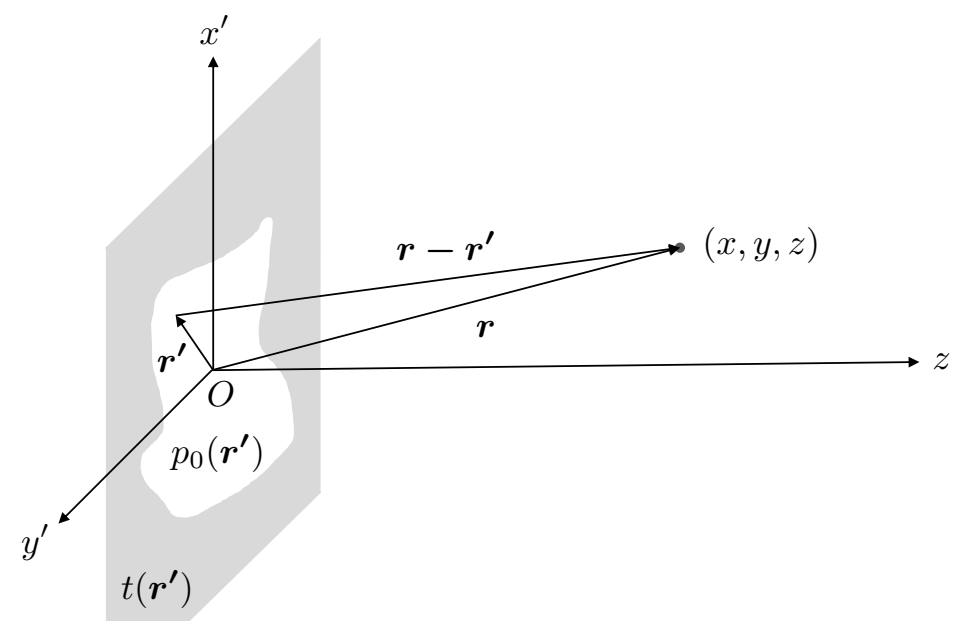

Figure 2.1: Schematic view of an aperture with an incident pressure field $p_{0}\left(\boldsymbol{r}^{\prime}\right)$. Gray areas indicate pressure opaque areas $\left(t\left(\boldsymbol{r}^{\prime}\right)=0\right)$, while withe regions represent transparent zones $\left(t\left(\boldsymbol{r}^{\prime}\right)=1\right)$.

where $k=\omega / c=2 \pi / \lambda$ is the wavenumber, being $\lambda$ the wavelength and $c$ the speed of sound, and $S$ is the surface of the aperture. The transmission function of the aperture, $t\left(\boldsymbol{r}^{\prime}\right)$, is 1 at the transparent areas of the aperture, and 0 at the opaque regions. The distance between a point $\boldsymbol{r}^{\prime}$ at the aperture and a given point $\boldsymbol{r}$ beyond the aperture, can be expressed in cartesian coordinates as

$$
\left\|\boldsymbol{r}-\boldsymbol{r}^{\prime}\right\|=\sqrt{\left(x-x^{\prime}\right)^{2}+\left(y-y^{\prime}\right)^{2}+z^{2}}
$$

Equation 2.1 can also be expressed as a convolution of the pressure field at the aperture with the so-called propagator kernel [1], $h(r)$, given by

$$
h(r)=\frac{e^{j k r}}{2 \pi r}\left(\frac{1}{r}-j k\right) \frac{z}{r},
$$

which results in 


$$
p(\boldsymbol{r})=\left[p_{0}(\boldsymbol{r}) t(\boldsymbol{r})\right] * h(\boldsymbol{r})=\iint_{\boldsymbol{r}^{\prime}} p_{0}\left(\boldsymbol{r}^{\prime}\right) t\left(\boldsymbol{r}^{\prime}\right) h\left(\boldsymbol{r}-\boldsymbol{r}^{\prime}\right) d \boldsymbol{r}^{\prime}
$$

where $(*)$ represents the convolution operator in the $x^{\prime} y^{\prime}$ plane.

Generally, the distance $\left\|\boldsymbol{r}-\boldsymbol{r}^{\prime}\right\|$ is much larger than the wavelength, so the term in the parentheses of equation 2.1 is usually approximated as $\left(1 /\left\|\boldsymbol{r}-\boldsymbol{r}^{\prime}\right\|-j k\right) \approx-j k[1]$, which yields to the simplified expression

$$
p(\boldsymbol{r})=\frac{-j k}{2 \pi} \iint_{S} p_{0}\left(\boldsymbol{r}^{\prime}\right) t\left(\boldsymbol{r}^{\prime}\right) \frac{e^{j k\left\|\boldsymbol{r}-\boldsymbol{r}^{\prime}\right\|}}{\left\|\boldsymbol{r}-\boldsymbol{r}^{\prime}\right\|} \frac{z}{\left\|\boldsymbol{r}-\boldsymbol{r}^{\prime}\right\|} d S,
$$

which is widely employed in the next chapters of this thesis to compute $2 \mathrm{D}$ pressure profiles. The term $z /\left\|\boldsymbol{r}-\boldsymbol{r}^{\prime}\right\|$ is usually called the obliquity factor, as it represents the cosine of the angle between the normal direction at the aperture surface and the vector $\boldsymbol{r}-\boldsymbol{r}^{\prime}$, and it is denoted by $\cos \theta$.

For axisymmetric apertures or lenses, such as Fresnel Zone Plates (FZPs), it is convenient to express the pressure fields in polar coordinates, that is, $\boldsymbol{r}^{\prime}=$ $\left(\rho^{\prime}, \varphi^{\prime}, 0\right)$ and $\boldsymbol{r}=(\rho, \varphi, z)$. In this situation, the distance $\left\|\boldsymbol{r}-\boldsymbol{r}^{\prime}\right\|$ becomes

$$
\left\|\boldsymbol{r}-\boldsymbol{r}^{\prime}\right\|=\sqrt{z^{2}+\rho^{2}+\rho^{\prime 2}-2 \rho \rho^{\prime} \cos \left(\varphi-\varphi^{\prime}\right)}
$$

and the incident pressure and transmission function can be simplified to $p_{0}\left(\boldsymbol{r}^{\prime}\right)=$ $p_{0}\left(\rho^{\prime}\right)$ and $t\left(\boldsymbol{r}^{\prime}\right)=t\left(\rho^{\prime}\right)$, as they do not present $\varphi^{\prime}$ dependence.

One of the most important characteristics of FZP lenses is their focusing profile, that is, the pressure distribution along the central axis of the lens, $\boldsymbol{r}=$ $(\rho=0, \varphi=0, z)$. In this case, keeping in mind that $d S=\rho^{\prime} d \rho^{\prime} d \varphi^{\prime}$, equation 2.5 can be expressed as

$$
p(z)=\frac{-j k z}{2 \pi} \int_{0}^{2 \pi} \int_{0}^{a} p_{0}\left(\rho^{\prime}\right) t\left(\rho^{\prime}\right) \frac{e^{j k \sqrt{z^{2}+\rho^{\prime 2}}}}{z^{2}+\rho^{\prime 2}} \rho^{\prime} d \rho^{\prime} d \varphi^{\prime}
$$

where $a$ is the maximum radius of the lens or the aperture.

Integrating the $d \varphi^{\prime}$ term in the above equation, finally yields to the simplified expression to compute the focusing profile of an axisymmetric lens: 


$$
p(z)=-j k z \int_{0}^{a} p_{0}\left(\rho^{\prime}\right) t\left(\rho^{\prime}\right) \frac{e^{j k \sqrt{z^{2}+\rho^{\prime 2}}}}{z^{2}+\rho^{\prime 2}} \rho^{\prime} d \rho^{\prime} .
$$

Further approximations can be made to the above expression if certain conditions are fulfilled. First, if the focal distance of the lens (or the computing points of the $z$-axis) is considerably longer than the size of the lens, the obliquity factor can be approximated as 1 , as the angle $\theta$ is very small. This allows to approximate the term $z / \sqrt{z^{2}+\rho^{\prime 2}} \approx 1$ in the above equation. Second, we can simplify the term $\sqrt{z^{2}+\rho^{\prime 2}}$ by considering the Taylor expansion of $\sqrt{1+x}$ [3], which is given by

$$
\sqrt{1+x}=1+\frac{x}{2}-\frac{x^{2}}{8}+\cdots
$$

Therefore, the term $\sqrt{z^{2}+\rho^{\prime 2}}$ can be approximated as

$$
\sqrt{z^{2}+\rho^{\prime 2}}=z \sqrt{1+\left(\frac{\rho^{\prime}}{z}\right)^{2}} \approx z+\frac{\rho^{\prime 2}}{2 z}-\frac{\rho^{\prime 4}}{8 z^{3}}
$$

The first two terms of the Taylor expansion provide an excellent approximation when $z^{3}>>a^{4} /(8 \lambda)$, which is a common scenario in most optical applications. The question here is whether to use only the first term or to consider both terms of the expansion. When $\sqrt{z^{2}+\rho^{\prime 2}}$ appears in the denominator as an amplitude term, using only the first term of the expansion provides a very good approximation. However, when $\sqrt{z^{2}+\rho^{\prime 2}}$ affects the phase of the complex exponential function, the first two terms provide a better approximation [3]. Keeping these two approximations in mind, equation 2.8 can be written as

$$
p(z)=-j k \frac{e^{j k z}}{z} \int_{0}^{a} p_{0}\left(\rho^{\prime}\right) t\left(\rho^{\prime}\right) e^{j k \frac{\rho^{\prime 2}}{2 z}} \rho^{\prime} d \rho^{\prime},
$$

which is known as the Fresnel diffraction expression [3].

These approximations are usually valid in optics, but in most ultrasound scenarios the approximations provide inaccurate results, and therefore equations 2.5 and 2.8 should be employed to compute the pressure of ultrasonic lenses. Nevertheless, discussing these approximations and their implications is very important, as it will be seen in the next chapters of this dissertation. Finally, we can perform 
a change of variable $\left(\rho^{\prime}, z\right) \rightarrow(\xi, u)$, where $u=\frac{a^{2}}{2 \lambda z}$ is the normalized axial coordinate and $\xi=\left(\rho^{\prime} / a\right)^{2}$ is the normalized radial coordinate. With this change, equation 2.11 becomes

$$
p(u)=-j 2 \pi u e^{j k \frac{a^{2}}{2 \lambda u}} \int_{0}^{1} p_{0}(\xi) t(\xi) e^{j 2 \pi \xi u} d \xi
$$

meaning that the focusing profile of the lens, in the normalized coordinates, can be obtained as a Fourier transform of the input pressure radial distribution.

Here, it is worth noting that, generally, focusing profiles and 2D maps are shown in power-related units. Therefore, all the results are usually given as a function of the acoustic intensity, which can be directly calculated from the pressure as

$$
I(\boldsymbol{r})=\frac{\|p(\boldsymbol{r})\|^{2}}{2 \rho_{0} c_{0}},
$$

being $c_{0}$ and $\rho_{0}$ the speed of sound and the density in the medium, respectively. The acoustic intensity is measured in $\mathrm{W} / \mathrm{m}^{2}$.

\subsection{Propagation}

If the $2 \mathrm{D}$ pressure field is known at a distance $z_{0}, p\left(x, y, z_{0}\right)$, the field can be propagated to any given $z$-axis distance by directly using the Rayleigh-Sommerfeld integral described in the previous section (equation 2.1). However, performing the 2D integral can be computationally expensive for large domains or for a big number of $z$-axis positions. In this sense, the angular spectrum approach (ASA) becomes a very useful tool to propagate pressure fields [1, 3], as it is based on performing fast Fourier transforms (FFTs). A convolution can be expressed in the frequency domain as a conventional product, and therefore equation 2.4 can be expressed as

$$
P\left(f_{x}, f_{y}, z\right)=P\left(f_{x}, f_{y}, z_{0}\right) H\left(f_{x}, f_{y}, \Delta z\right)
$$

where $P\left(f_{x}, f_{y}, z_{0}\right)$ represents the angular spectrum of the pressure field, which is a decomposition of the pressure wavefront into a combination of plane waves with different angles, and $H\left(f_{x}, f_{y}, \Delta z\right)$ represents the propagator kernel from $z_{0}$ to $z$ 
in the spatial frequency domain. The angular spectrum is given as a 2D Fourier transform in the $x y$ plane, that is,

$$
P\left(f_{x}, f_{y}, z_{0}\right)=\int_{-\infty}^{+\infty} \int_{-\infty}^{+\infty} p\left(x, y, z_{0}\right) e^{-j 2 \pi f_{x} x} e^{-j 2 \pi f_{y} y} d x d y
$$

The propagation kernel is given by

$$
H\left(f_{x}, f_{y}, \Delta z\right)= \begin{cases}e^{j\left(z-z_{0}\right) \sqrt{k^{2}-\left(k_{x}^{2}+k_{y}^{2}\right)}}, & k_{x}^{2}+k_{y}^{2} \leq k^{2} \\ e^{-\left(z-z_{0}\right) \sqrt{\left(k_{x}^{2}+k_{y}^{2}\right)-k^{2}}}, & k_{x}^{2}+k_{y}^{2}>k^{2}\end{cases}
$$

being $k_{x}=2 \pi f_{x}$ and $k_{y}=2 \pi f_{y}$. In the region $k_{x}^{2}+k_{y}^{2}>k^{2}$, the propagator kernel decays exponentially, which is associated with evanescent waves.

Once $P\left(f_{x}, f_{y}, z\right)$ is obtained, the pressure distribution can be computed as an inverse Fourier transform:

$$
p(x, y, z)=\int_{-\infty}^{+\infty} \int_{-\infty}^{+\infty} P\left(f_{x}, f_{y}, z\right) e^{j 2 \pi f_{x} x} e^{j 2 \pi f_{y} y} d f_{x} d f_{y} .
$$

Both Rayleigh-Sommerfeld and ASA techniques provide identical results, but computation times are considerably lower for the angular spectrum method when propagating or backpropagating pressure fields to a big number of $z$-axis positions.

\subsection{Pressure Radiation from a Circular Planar Trans- ducer}

Planar ultrasound transducers with circular geometry, also known as piston transducers, are one of the most common devices used to generate pressure fields in acoustics. As piston sources are employed throughout this thesis during experimental measurements, it is worth analyzing the main theoretical expressions that can model the pressure field generated by this kind of transducers. The pressure 
field generated by a circular piston source of radius $a$ at a point $\boldsymbol{r}=(x, y, z)$ in space, vibrating with a time-harmonic normal velocity of $u(t)=u_{0} e^{j \omega t}$, and mounted on a rigid baffle, can be analyzed using the Rayleigh integral $[2,4]$,

$$
p(\boldsymbol{r}, t)=\frac{j k \rho_{0} c_{0} u_{0} e^{j \omega t}}{2 \pi} \iint_{S^{\prime}} \frac{e^{-j k\left\|\boldsymbol{r}-\boldsymbol{r}^{\prime}\right\|}}{\left\|\boldsymbol{r}-\boldsymbol{r}^{\prime}\right\|} d S^{\prime}
$$

where $k=\omega / c_{0}$ is the wavenumber, $\rho_{0}$ and $c_{0}$ are the density and speed of sound in the medium, and $\boldsymbol{r}^{\prime}=\left(x^{\prime}, y^{\prime}, 0\right)$ is a point inside the piston surface $S^{\prime}$. We can omit the harmonic term $e^{j \omega t}$ for simplicity, as $p(\boldsymbol{r}, t)=p(\boldsymbol{r}) e^{j \omega t}$, and focus on the term $p(\boldsymbol{r})$. Keeping in mind that $Z_{0}=\rho_{0} c_{0}$ is the acoustic impedance of the medium, we can simplify the term in the numerator $\rho_{0} c_{0} u_{0}=Z_{0} u_{0}=p_{0}$, where $p_{0}$ represents the normal pressure at the piston surface.

In the far-field distance, where $r>>a$, the Rayleigh integral of equation 2.18 can be calculated as $[2,4]$

$$
p(r, \theta)=\frac{j p_{0} a}{r} \frac{J_{1}(k a \sin \theta)}{\sin \theta} e^{-j k r}
$$

being $r=\|\boldsymbol{r}\|, J_{1}(\cdot)$ the first kind and first order Bessel function, and $\theta$ the angle measured from the normal direction of the piston surface. One important parameter of any transducer is its directivity pattern, which indicates how the transducer distributes the radiated energy depending on the radiation angle $\theta$. The directivity pattern, $D(\theta)$, can be calculated as the ratio between the pressure radiated at any given angle and the pressure radiated at $\theta=0$ (normal direction), that is,

$$
D(\theta)=\frac{p(r, \theta)}{p(r, 0)}
$$

which in this case results in

$$
D(\theta)=\frac{2 J_{1}(k a \sin \theta)}{k a \sin \theta}
$$

Higher $k a=2 \pi a / \lambda$ values (i.e. transducers with a high aperture in terms of the wavelength) result in narrower directivity patterns, which means that the radiated energy from the piston is more spatially concentrated around the normal 
direction to the transducer surface. On the other hand, transducers with low $k a$ generate wider radiation patterns and distribute the radiated energy more evenly across every angular direction.

Finally, the radiated pressure from a piston transducer can be expressed in the far-field as

$$
p(r, \theta)=\frac{j k p_{0} a^{2}}{2 r} D(\theta) e^{-j k r}
$$

\subsection{Numerical Simulations}

Diffraction and propagation simulations of pressure fields generated by FZPs were carried out by numerically computing the Rayleigh-Sommerfeld integral and the angular spectrum method, respectively. As the manufactured FZPs are thin enough in terms of the wavelength, the transmission function can be approximated as a 2D transmission function, and both Rayleigh-Sommerfeld and ASA techniques provide results in excellent agreement with experimental measurements. When the Rayleigh-Sommerfeld method is employed, equations 2.5 and 2.8 are numerically integrated in MATLAB (The MathWorks, Inc.), using the trapezoidal technique. The integration grid size is set to $\lambda / 20$ for the $1 \mathrm{D}$ case, and $\lambda / 10$ for the $2 \mathrm{D}$ case. Computing times range from a few seconds for the 1D case, to a few minutes for the $2 \mathrm{D}$ case. Propagation simulations using ASA are based on performing FFTs and IFFTs, and computation times are in the order of a few seconds.

On the other hand, simulations of more complex structures, including wave propagation in solids and simulations of liquid-filled spherical lenses, were carried out using the finite element method (FEM) in COMSOL Multiphysics (COMSOL, Inc.). Acoustic-structure multiphysics interfaces are used to account for the wave coupling between liquid and solid media. A triangular mesh is employed for 2D and 2D-axisymmetric models, while a tetrahedral mesh is employed for $3 \mathrm{D}$ simulations. The maximum mesh size is set to $\lambda / 8$, being $\lambda$ the wavelength at each medium, which ensures a good convergence of the solution. In the case of spherical liquid-filled lenses, as the shell is very thin compared to the wavelength, the mesh size is set to $t_{h} / 10$ at the shell domain (where $t_{h}$ represents the thickness of the shell), to ensure at least 10 simulation points in that region. A pressure condition is employed to model the ultrasound transducer, while plane wave radiation conditions or perfectly matched layers (PMLs) are used to avoid reflections inside the computation domain. Computation times range from a few minutes for simple models, to hours for more complex simulations. 

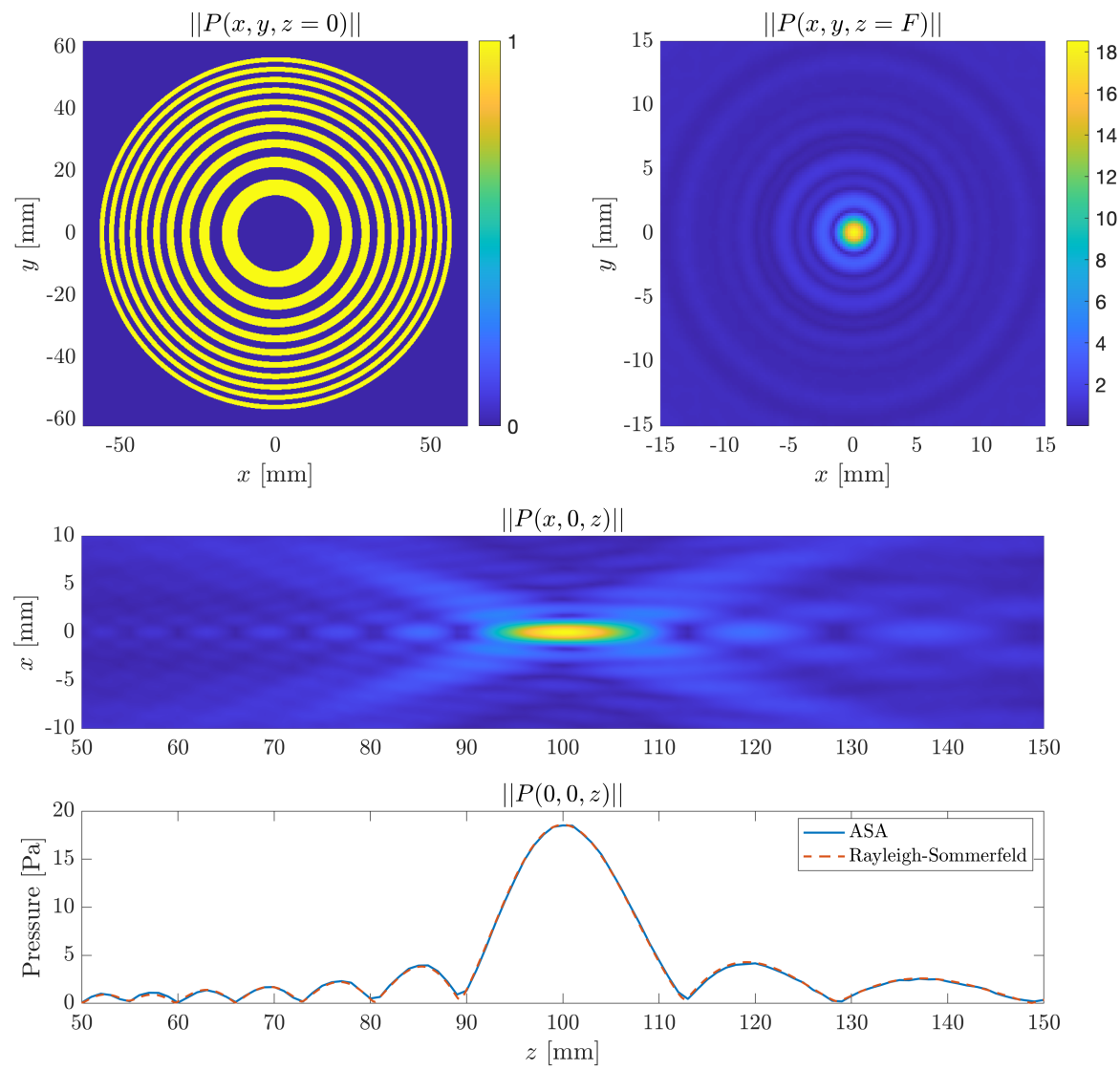

Figure 2.2: Example of a simulation computed with ASA and Rayleigh-Sommerfeld techniques. Pressure field at the apertures of an FZP lens (top left) and propagated pressure field at its focal distance using ASA (top right). Computed 2D pressure map with ASA (middle) and axial pressure distribution along the central axis of the lens (bottom). As can be observed from the bottom plot, both RayleighSommerfeld and ASA computations are in excellent agreement.

\subsection{Experimental Measurements}

All experimental measurements carried out in this thesis were performed using the same experimental set-up. The set-up consists of a custom made 3-axis robotic system with a spatial scanning resolution of $1 \times 1 \times 1 \mathrm{~mm}^{3}$ (Artitecnic S.L.). The measurement process is controlled using a National Instruments NI-7330 controller 


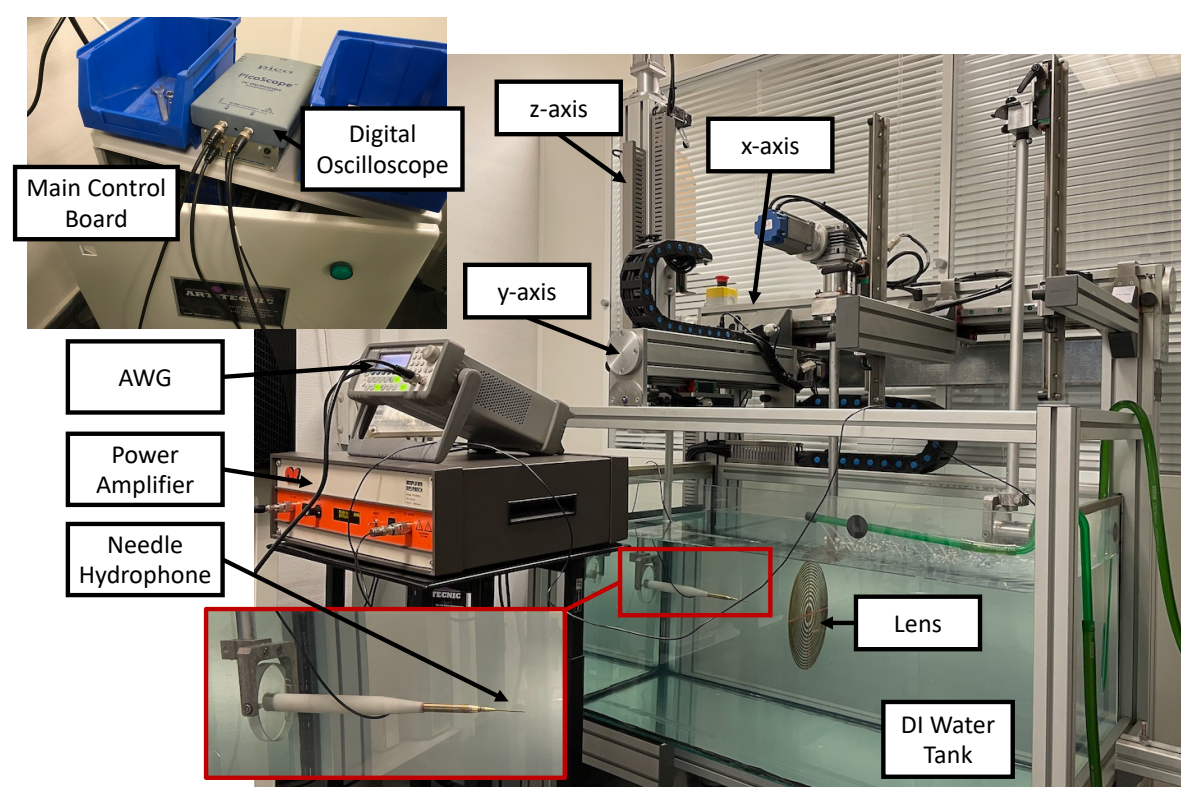

Figure 2.3: Picture of the experimental set-up with an FZP lens.

card and a custom LabView software developed by CPOH-UPV, which performs the spatial scanning with the robotic arm and the acoustic waveform acquisition at each point. The water tank dimensions are $0.5 \times 0.5 \times 1 \mathrm{~m}^{3}$, and the tank is filled with deionized (DI) water.

The excitation signal of the transducers is generated using two different configurations, depending on the requirements of each experiment. The first configuration consists of using an Olympus 5077PR pulser, with a negative square waveform of up to $-400 \mathrm{~V}$ peak voltage, and a pulse repetition frequency that ranges from $100 \mathrm{~Hz}$ to $5 \mathrm{kHz}$. The second configuration consists of an arbitrary waveform generator (AWG) connected to a solid-state $75 \mathrm{~W}$ RF power amplifier (model 75A250A, Amplifier Research), with a working bandwidth from $10 \mathrm{kHz}$ to $250 \mathrm{MHz}$. The AWG is an Agilent 33220A, with a maximum frequency of 20 $\mathrm{MHz}$, capable of generating waveforms with up to 64000 points. The AWG is controlled with a MATLAB interface via USB, which generates and uploads the custom waveform to the AWG.

Three transducers have been employed throughout this thesis, with different central frequencies and active diameters. For acoustics lenses in the 250 $\mathrm{kHz}$ range, a $250 \mathrm{KHz}$ Imasonic piston transducer is used, with a $30 \mathrm{~mm}$ active 
diameter and a $180 \mathrm{kHz}$ bandwidth. For acoustic lenses in the $500 \mathrm{kHz}$, a 500 $\mathrm{kHz}$ Olympus transducer is employed, with a $360 \mathrm{kHz}$ bandwidth and an active diameter of $38 \mathrm{~mm}$. Finally, acoustic lenses in the $1 \mathrm{MHz}$ range are characterized using a $1 \mathrm{MHz}$ Olympus transducer, with an active diameter of $13 \mathrm{~mm}$.

The ultrasound waves are measured using a PVDF needle hydrophone from Precision Acoustics, with a physical diameter of $1.5 \mathrm{~mm}$, an active sensing diameter of $1 \mathrm{~mm}$, and a working frequency range from $200 \mathrm{kHz}$ to $15 \mathrm{MHz}$. The needle hydrophone is connected to a low-noise preamplifier, and then to a DC coupler. The system has an average sensitivity of $850 \mathrm{nV} / \mathrm{Pa}$. The signals are digitized using a PicoScope 3224 digital oscilloscope, connected via USB to the main computer. The oscilloscope can digitize up to 2 channels with a vertical resolution of 12 bits and a $72 \mathrm{~dB}$ dynamic range, with a maximum frequency of $10 \mathrm{MHz}$. Once the measurement process has finished, additional processing and post-processing tasks are performed using MATLAB. Conventional processing tasks include performing bandpass filtering for noise reduction, using the Hilbert transform for envelope detection, and applying the Fourier transform to analyze the lens response at each frequency.

\section{References}

[1] Dong-Lai Liu and R. Waag, "Propagation and backpropagation for ultrasonic wavefront design," IEEE Transactions on Ultrasonics, Ferroelectrics and Frequency Control, vol. 44, pp. 1-13, jan 1997.

[2] D. T. Blackstock, Fundamentals of Physical Acoustics. John Wiley \& Sons, Inc., 1st ed., 2000.

[3] J. W. Goodman, Introduction to Fourier Optics. The McGraw-Hill Companies, Inc., 4th ed., 2017.

[4] L. E. Kinsler, A. R. Frey, A. B. Coppens, and J. V. Sanders, Fundamentals of Acoustics. John Wiley \& Sons, Inc., 4th ed., 2000. 


\title{
Chapter 3
}

\section{On the use of Phase Correction Rings on Fresnel Zone Plates with Ultrasound Piston Emitters}

\author{
S. Pérez-López, J. M. Fuster, P. Candelas, C. Rubio, and F. Bel- \\ mar, "On the use of phase correction rings on Fresnel zone plates \\ with ultrasound piston emitters," Applied Physics Letters, vol. \\ 112, no. 26, p. 264102, Jun. 2018. \\ https://doi.org/10.1063/1.5036712
}

\section{Abstract}

In this work, the distortion of the Fresnel zone plate (FZP) focusing profile generated by a piston emitter in ultrasound applications is significantly reduced through the use of phase correction rings, which compensate the effect of the piston emitter radiation diagram. Both simulations and experimental results successfully demonstrate the improvement achieved with this design method over the conventional case. 


\subsection{Introduction}

Wave focusing is a hot topic of great interest in many areas of physics, including optics, X-rays, microwaves or ultrasounds [1-4]. Fresnel Zone Plates (FZPs) have been proposed for implementing this application in situations where conventional lenses are difficult to implement or planar fabrication is advantageous [5].

Conventional FZPs are capable of focusing a plane wave into a certain location with a single focus. The structure of the FZP is a set of concentric rings with increasing radius, each ring being a Fresnel region. The radius and width of each Fresnel ring depends on certain design parameters such as the desired focal length and the signal wavelength.

The use of FZPs in the ultrasound domain is very appealing, because it provides a high flexibility in certain medical applications where fast and accurate focus shifting is critical [6], such as tumour ablation [7-9]. Lenses based on passive focusing with acoustic metamaterials have also been addressed [10], providing new applications, such as acoustic cloacking. However, FZPs provide an easier to build and cheaper solution than lenses based on metametrials for conventional focusing applications.

Other approaches, such as multi-array devices [11, 12], have also been proposed, but they are complex and expensive systems that require Magnetic Resonance Imaging (MRI) real-time monitoring to adjust the amplitudes and phases of the emitting elements in order to focus at the intended treatment area. Therefore, the use of single-element focusing devices, such as FZPs, is a more attractive alternative, as they are cheaper and simpler to use.

Plane and spherical wave incidence have been extensively studied [4], and FZPs can be easily designed for such excitations. However, ultrasonic FZPs require the use of piston emitters [13-15], which in certain situations introduces a significant distortion on the focusing profile. This work demonstrates that an accurate focusing profile is achieved when the piston emitter parameters are included in the design process of ultrasonic FZPs.

\subsection{Results}

The governing equation for designing FZPs for point source excitation is given by, 


$$
d+F+n \frac{\lambda}{2}=\sqrt{d^{2}+r_{n}^{2}}+\sqrt{F^{2}+r_{n}^{2}}
$$

with $n=1,2, \ldots, N$, being $N$ the number of Fresnel regions, $F$ the FZP focal distance, $\lambda$ the operating wavelength, $d$ the separation between the point source and the FZP, and $r_{n}$ the radius of each Fresnel region. Using equation 3.1, the $r_{n}$ values are obtained from the FZP design parameters $(F, N, \lambda$ and $d)$.

Figure 3.1 shows a setup with an FZP placed at a distance $d$ of a piston emitter in the ultrasound domain, with $F$ being the FZP focal distance. In this situation, in the far field case, the piston can be modelled as a point source emitter with a specific directivity pattern, given by,

$$
D(\theta)=\frac{2 J_{1}(k a \sin (\theta))}{k a \sin (\theta)}
$$

with $k$ being the wavenumber, $a$ the piston active radius, $\theta$ the angle referenced to the normal direction of the piston surface, and $J_{1}$ the first kind and first order Bessel function.

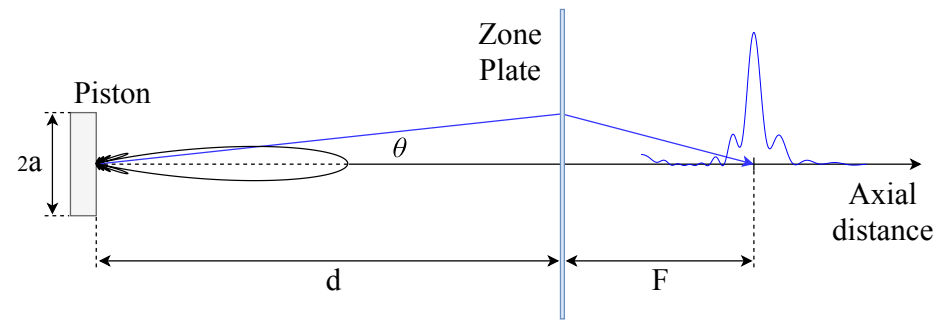

Figure 3.1: Setup of an FZP with a piston emitter in ultrasound applications.

Figure 3.2 shows the directivity of a piston with a combined $k a$ product of 16.96. As can be observed, the piston introduces two drawbacks over the ideal point source emitter: the non-omnidirectional behaviour and the $\pi$-phase change at some secondary lobes. This second drawback is more significant, because the additional $\pi$-phase shift introduced in the secondary lobes causes that their contributions destructively interfere those of the main lobe, which results in a significant distortion at the focusing profile. Thus, the maximum angle $\theta_{\max }$ beyond which there would be a phase error due to the piston directivity diagram can be calculated from the first zero of the $J_{1}(x)$ function, which is located at $x=3.83$, and is given by, 


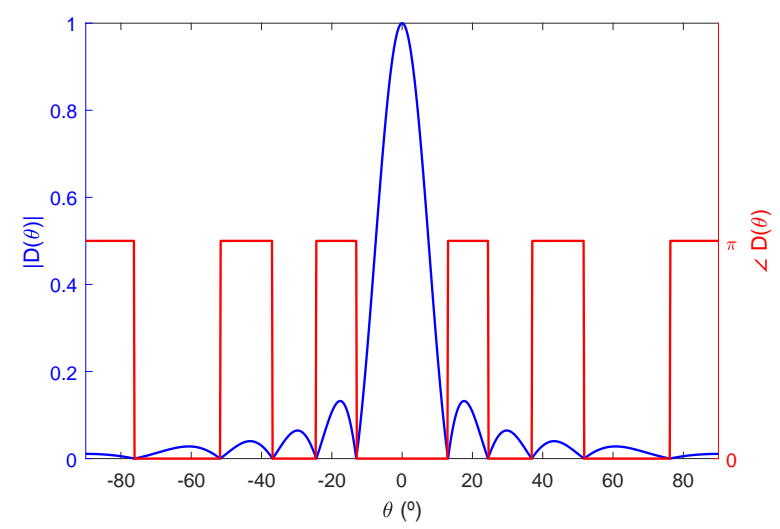

Figure 3.2: Directivity of a piston with $k a=16.96$; module (blue) and phase (red).

$$
\theta_{\max }=\arcsin \left(\frac{3.83}{k a}\right)
$$

Using the $\theta_{\max }$ obtained from equation 3.3 , it is possible to calculate the maximum FZP radius $\left(R_{\max }\right)$ as,

$$
R_{\max }=d \tan \left(\theta_{\max }\right)
$$

Equations 3.1 and 3.4 yield to a maximum number of radii for the FZP in order to avoid phase distortion:

$$
N_{\max }=\left\lfloor\frac{2}{\lambda}\left(\sqrt{d^{2}+R_{\max }^{2}}+\sqrt{F^{2}+R_{\max }^{2}}-d-F\right)\right\rfloor,
$$

with $\lfloor\cdot\rfloor$ being the floor operator.

If $N>N_{\max }$ the $\pi$-phase error introduced by the piston first secondary lobe results in a destructive interference at the focal distance, as mentioned before. Figure 3.3 shows the computed axial intensity of the same FZP with $N_{\max }=14$ for both an ideal point source emitter (blue line) and a piston emitter with $k a=16.96$ (red line). As can be observed from the figure, the phase error generates a very 
noticeable distortion on the focusing profile, which can yield into a malfunctioning of the FZP. It is worth noting that at the focal distance, the acoustic intensity shifts from a maximum value in the point emitter case to a local minimum in the piston case.

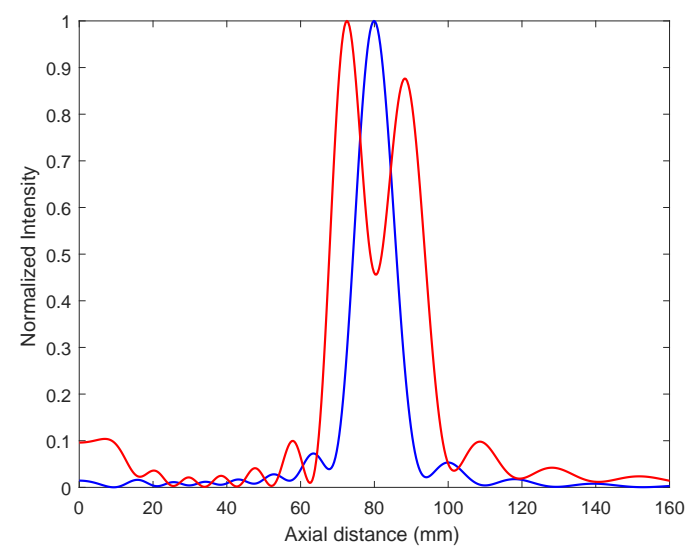

Figure 3.3: Normalized axial intensity of a FZP with $N=31, F=80 \mathrm{~mm}$ and $\lambda=5.55 \mathrm{~mm}$. Ideal point source emitter (blue line) and piston emitter with $k a=16.96$ and $d=340 \mathrm{~mm}$ (red line).

The interference behaviour of each Fresnel zone can be analysed by plotting the directivity phase and the FZP radii in the same graph, as shown in Figure 3.4. Thus, those Fresnel zones in which the piston phase is $\pi$ generate a destructive interference, whereas those radii in which the piston phase is 0 generate a constructive interference.

In order to correct the phase error, a phase correction ring (PCR) must be added to the FZP when $N>N_{\max }$ so the $\pi$-phase change is compensated. The PCR consists of skipping one Fresnel radius when the $n>N_{\max }$ condition is reached in equation 3.1, therefore introducing an additional phase shift of $\pi$ that compensates the piston phase error. This PCR should be added every time the FZP radius changes from a piston directivity lobe to the next one.

Figure 3.5 shows two different FZPs using piston emitters, with the same number of Fresnel regions $(N=31)$, and their corresponding focusing profiles. Figures 3.5(a) and 3.5(c) correspond to a traditional FZP, while 3.5(b) and 3.5(d) are associated with the new FZP design with one PCR. In both simulations $k a=16.96$ and the distance between the piston and the FZP is $340 \mathrm{~mm}$. As can be observed form the comparison of Figures 3.5(c) and 3.5(d), the PCR suc- 


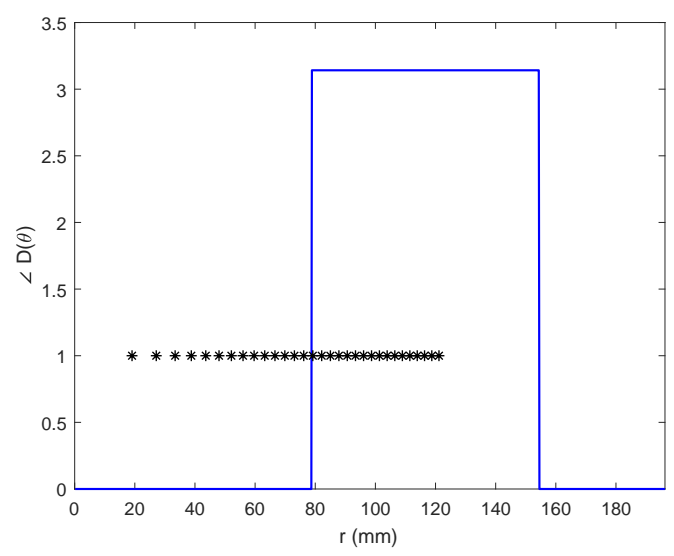

Figure 3.4: FZP radii (black) and directivity phase for a piston with $k a=16.96$ and $d=340 \mathrm{~mm}$ (blue), $N=31, F=80 \mathrm{~mm}$ and $\lambda=5.55 \mathrm{~mm}$.

cessfully removes the phase error introduced by the piston and achieves a focal distance of $80 \mathrm{~mm}$, which corresponds to the theoretically designed focal distance. However, there is still some remaining distortion introduced by the piston due to the amplitude of its directivity pattern, that cannot be compensated and increases the level of the side lobes adjacent to the main focus.

Figure 3.6 shows additional simulation results for two different focal distances, $F=60 \mathrm{~mm}$ (Figure 3.6(a) and Figure 3.6(b)) and $F=100 \mathrm{~mm}$ (Figure 3.6(c) and Figure 3.6(d)). Comparing the focusing profiles corresponding to the three different focal distances shown in Figures 3.5 and 3.6, it can be deduced that the distortion increases with the focal distance. In the conventional FZP case, the increase in distortion can be observed in the destructive interference at the focal distance, which becomes more significant as the focal length increases. In the FZP-PCR case, when the focal length augments, the side lobes become higher. In this case, the overall distortion is much lower, because phase distortion has been removed with the PCR. However, the remaining amplitude distortion augments with the focal distance, increasing the level of the side lobes adjacent to the main focus.

In order to evaluate the distortion introduced by the piston, Figure 3.7 shows the computed acoustic intensity at the focal distance as a function of $N$ for three different FZPs: a conventional FZP (red line), a FZP with one PCR (blue line) and a FZP with two PCRs (black line). The boundary between the main lobe and the first secondary lobe is located at $N_{\max }^{1}=14$ and the boundary between 
(a)

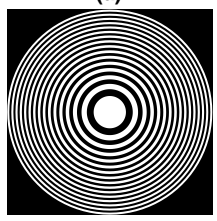

(c)

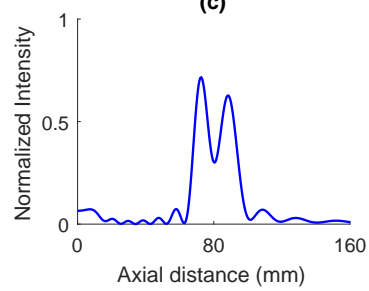

(b)

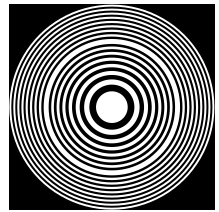

(d)

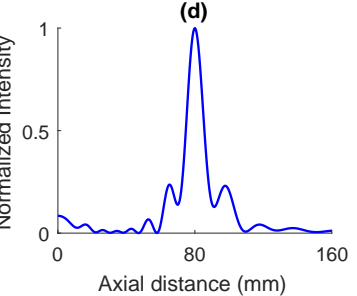

Figure 3.5: (a) Conventional FZP layout and (c) its normalized axial intensity. (b) FZP with PCR and (d) its normalized axial intensity; $N=31, F=80 \mathrm{~mm}$, $\lambda=5.55 \mathrm{~mm}, k a=16.96$ and $d=340 \mathrm{~mm}$.

(a)

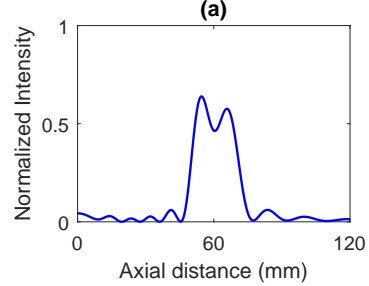

(c)

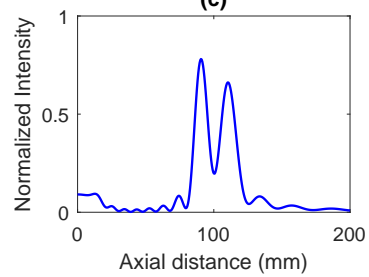

(b)

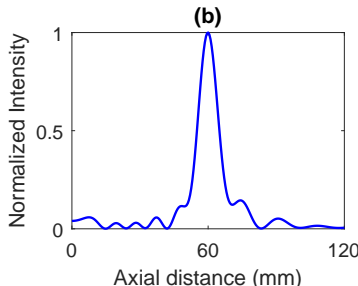

(d)

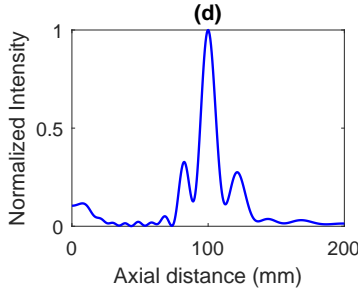

Figure 3.6: Axial intensity profiles: (a) conventional FZP with $F=60 \mathrm{~mm}$, (b) FZP with PCR and $F=60 \mathrm{~mm}$, (c) conventional FZP with $F=100 \mathrm{~mm}$, (d) FZP with PCR and $F=100 \mathrm{~mm} ; N=31, \lambda=5.55 \mathrm{~mm}, k a=16.96$ and $d=340 \mathrm{~mm}$.

the first secondary lobe and the next one is located at $N_{\max }^{2}=45$. As can be 


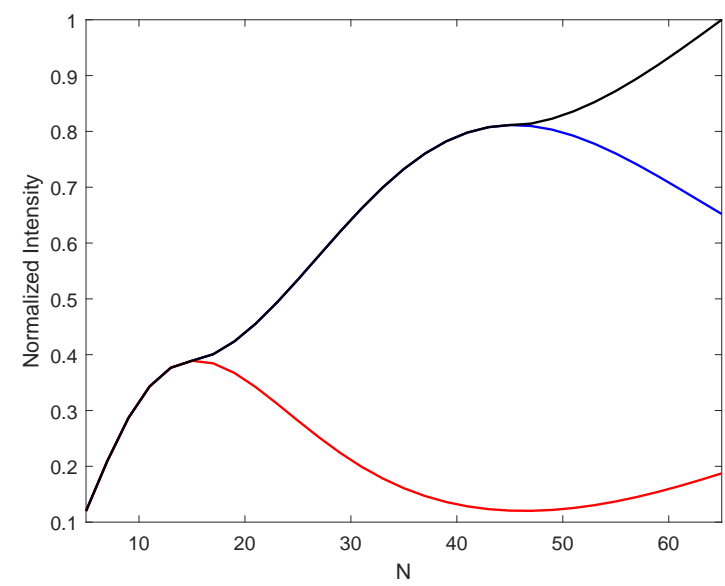

Figure 3.7: Normalized intensity at focal distance: Conventional FZP (red), FZP with one PCR (blue) and FZP with two PCRs (black). $k a=16.96, d=340 \mathrm{~mm}$, $N_{\max }^{1}=14$ and $N_{\max }^{2}=45$.

observed from Figure 3.7, when $N>14$ the focal intensity of the conventional FZP does not increase when $\mathrm{N}$ increases as expected, but instead it begins to decrease due to the destructive interferences generated by the $N_{\max }^{1}<N<N_{\max }^{2}$ Fresnel zones. The FZP with one PCR compensates the phase error up to $N=N_{\max }^{2}$. As this FZP does not have the second PCR, when $N>45$ the intensity at the focal distance begins to decrease, while it starts to increase again for the conventional case. This phenomena is due to the fact that for $N>45$ the phase of the piston directivity pattern is not $\pi$ but 0 again, due to the change to the next secondary lobe. Finally, the last FZP includes two PCRs for compensating the phase error due to both transitions in the piston phase diagram and presents a proper behaviour. In this last case, the intensity at the focus is continuously increasing because all the contributions are constructive. This mechanism can be extended, adding additional PCRs when required, if the number of Fresnel zones increases and more piston side lobes contribute to the FZP focusing profile.

The simulation results have been validated through experimental measurements. Figure 3.8 shows the experimental set-up. An underwater 3D automated positioning system with a spatial resolution of $1 \times 1 \times 1 \mathrm{~mm}^{3}$ is used inside a water tank. An Imasonic piston transducer with a $30 \mathrm{~mm}$ active diameter, a central frequency of $260.88 \mathrm{kHz}$ and a $-6 \mathrm{~dB}$ bandwidth of $180.66 \mathrm{kHz}$ is used as emitter. A needle hydrophone from Precision Acoustics Ltd. with a diameter of $1.5 \mathrm{~mm}$ and a $-4 \mathrm{~dB}$ bandwidth that goes from $200 \mathrm{kHz}$ to $25 \mathrm{MHz}$ is used as receiver. 
The transmitted signal is generated using a Panametrics 5077PR pulser, whereas the received signal is acquired and sampled using a digital oscilloscope from Pico Technology with 12-bit resolution and $72 \mathrm{~dB}$ dynamic range.

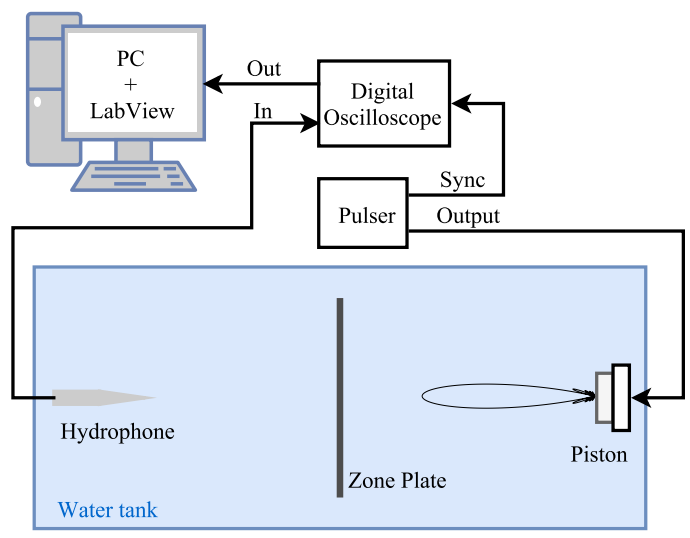

Figure 3.8: Experimental set-up.

Two Soret FZPs have been measured in the experiment, a conventional FZP (Figure 3.9(a)) and the novel FZP with a PCR (Figure 3.9(b)). The FZP external radii are $R_{L}=122.11 \mathrm{~mm}$ and $R_{L}=123.51 \mathrm{~mm}$, respectively. Both lenses are very similar in size, as they differ in a single Fresnel zone. The FZPs are $1 \mathrm{~mm}$ thick and made of brass. They have been designed for operating at 270 $\mathrm{kHz}$ at a focal distance of $80 \mathrm{~mm}$. The number of Fresnel zones is 31 and the separation between the piston and the zone plate is $340 \mathrm{~mm}$. The water sound speed is $c_{w}=1500 \mathrm{~m} / \mathrm{s}$.

(a)

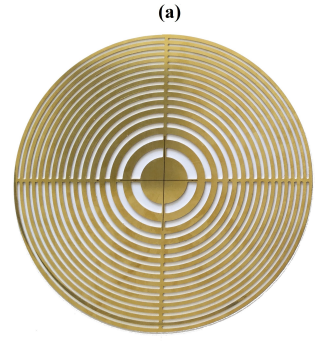

(b)

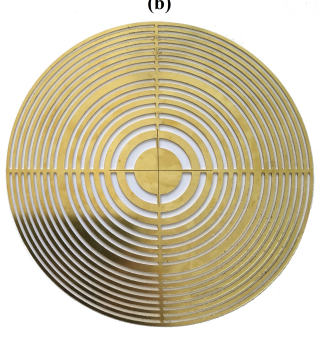

Figure 3.9: (a) Conventional FZP with $R_{L}=122.11 \mathrm{~mm}$ (b) FZP-PCR with $R_{L}=123.51 \mathrm{~mm}$.

Figure 3.10 shows the acoustic intensity maps for both the conventional FZP $(3.10(a))$ and the novel FZP with one PCR (3.10(b)). Both maps have been 
normalized to the same reference, the maximum value in the FZP-PCR case. As can be observed from the figure, the focal distance of the FZP with one PCR agrees accurately with the design value of $80 \mathrm{~mm}$, while the focusing profile of the conventional FZP is severely distorted, and its main focus is shifted $10 \mathrm{~mm}$ towards the lens plane.
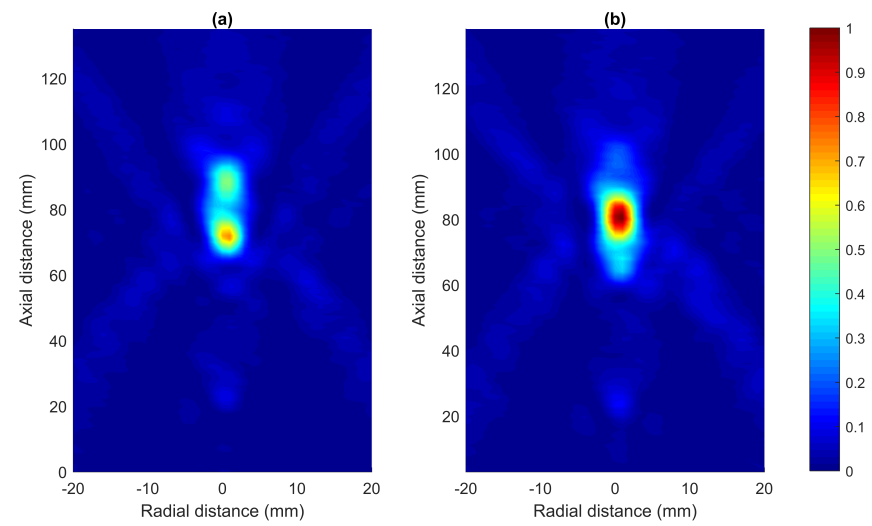

Figure 3.10: Experimental acoustic intensity maps: conventional FZP (a) and FZP with PCR (b).

Figure 3.11 shows the measured focusing profiles along the axial direction for both lenses; conventional FZP (blue) and FZP with one PCR (red). The corresponding simulation results are also depicted as dashed lines. As can be observed from Figure 3.11, experimental results do agree very well with simulations, and the FZP with the correction ring achieves its theoretical focal distance of $80 \mathrm{~mm}$, whereas the standard FZP presents destructive interference at that same focal distance.

Figure 3.12 shows the measured focusing profiles along the transverse direction for both lenses; conventional FZP (blue) and FZP with one PCR (red). The corresponding simulation results are also depicted as dashed lines. As can be observed from Figure 3.11, experimental results do agree very well with simulations, and the FZP with one PCR presents a narrower beam waist ( $\omega_{0}=3.7$ $\mathrm{mm})$ than the conventional FZP $\left(\omega_{0}=5.6 \mathrm{~mm}\right)$. Both values are higher than the theoretical minimum beam waist value [16], which in this case corresponds to $\omega_{0}=2.3 \mathrm{~mm}$.

Although experimental and simulation results agree very well in Figures 3.11 and 3.12, there are small differences that can be produced by the cross-shaped mechanical support that can be observed in Figure 3.9, which maintains the FZP 
brass rings fixed in place. This cross-shaped structure has not been modeled in the simulation.

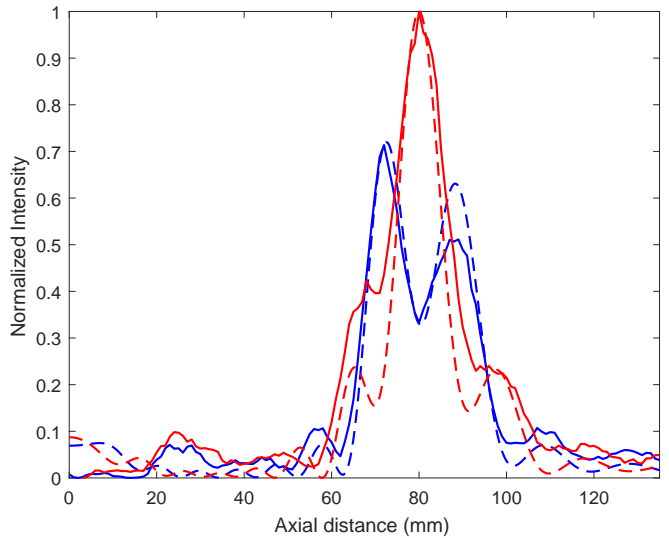

Figure 3.11: Axial focusing profiles for conventional FZP: measurements (blue solid line) and simulation (blue dashed line) and FZP with PCR: measurements (red solid line) and simulation (red dashed line).

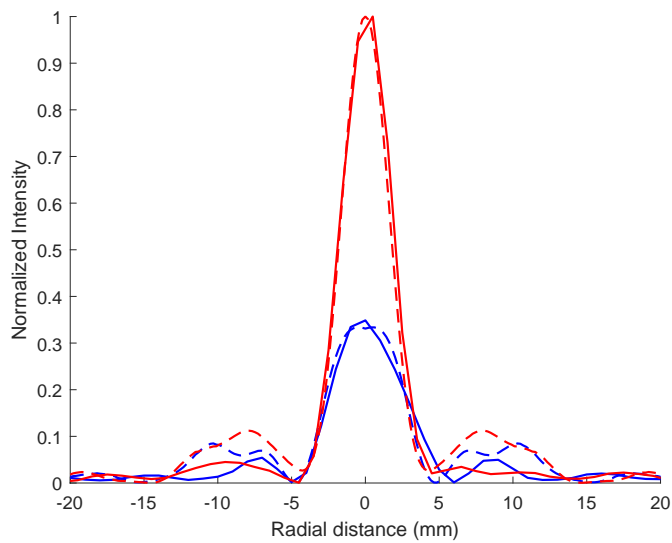

Figure 3.12: Radial focusing profiles for conventional FZP: measurements (blue solid line) and simulation (blue dashed line) and FZP with PCR: measurements (red solid line) and simulation (red dashed line). 


\subsection{Conclusions}

In this paper, a new method to design FZPs with piston emitters, which are typical for underwater ultrasound transmission, has been proposed. A theoretical analysis on the error introduced by the piston has been developed, and a method to eliminate the piston phase error based on PCRs has been proposed. Simulation results have been obtained to validate the theoretical model. Moreover, the performance of these new FZPs with PCRs has been experimentally demonstrated, showing an improved performance over conventional FZPs and removing the distortion on the focusing profile caused by the phase error introduce by piston emitters.

\section{References}

[1] S. Tamura, M. Yasumoto, N. Kamijo, A. Takeuchi, K. Uesugi, Y. Terada, and Y. Suzuki, "Quasi-blazed type multilayer zone plate for X-rays," Vacuum, vol. 84, no. 5, pp. 578-580, 2009.

[2] H. D. Hristov and J. M. Rodriguez, "Design equation for multidielectric fresnel zone plate lens," IEEE Microwave and Wireless Components Letters, vol. 22, no. 11, pp. 574-576, 2012.

[3] R. Yang, W. Tang, and Y. Hao, "A broadband zone plate lens from transformation optics," Optics Express, vol. 19, p. 12348, jun 2011.

[4] D. C. Calvo, A. L. Thangawng, M. Nicholas, and C. N. Layman, "Thin Fresnel zone plate lenses for focusing underwater sound," Applied Physics Letters, vol. 107, p. 014103, jul 2015.

[5] S. M. Stout-Grandy, A. Petosa, I. V. Minin, O. V. Minin, and J. Wight, "A Systematic Study of Varying Reference Phase in the Design of Circular Fresnel Zone Plate Antennas," IEEE Transactions on Antennas and Propagation, vol. 54, pp. 3629-3637, dec 2006.

[6] J. Fuster, P. Candelas, S. Castiñeira-lbáñez, S. Pérez-López, and C. Rubio, "Analysis of Fresnel Zone Plates Focusing Dependence on Operating Frequency," Sensors, vol. 17, p. 2809, dec 2017.

[7] J. Kennedy, F. Wu, G. ter Haar, F. Gleeson, R. Phillips, M. Middleton, and D. Cranston, "High-intensity focused ultrasound for the treatment of liver tumours," Ultrasonics, vol. 42, pp. 931-935, apr 2004.

[8] D. Suo, Z. Jin, X. Jiang, P. A. Dayton, and Y. Jing, "Microbubble mediated dual-frequency high intensity focused ultrasound thrombolysis: An In vitro study," Applied Physics Letters, vol. 110, p. 023703, jan 2017. 
[9] D. Suo, S. Guo, W. Lin, X. Jiang, and Y. Jing, "Thrombolysis using multifrequency high intensity focused ultrasound at $\mathrm{MHz}$ range: an in vitro study," Physics in Medicine and Biology, vol. 60, pp. 7403-7418, sep 2015.

[10] C. Shen, J. Xu, N. X. Fang, and Y. Jing, "Anisotropic complementary acoustic metamaterial for canceling out aberrating layers," Physical Review $X$, vol. 4, no. 4, 2014.

[11] A. Carovac, F. Smajlovic, and D. Junuzovic, "Application of Ultrasound in Medicine," Acta Informatica Medica, vol. 19, no. 3, p. 168, 2011.

[12] Y. Xie, C. Shen, W. Wang, J. Li, D. Suo, B.-I. Popa, Y. Jing, and S. A. Cummer, "Acoustic Holographic Rendering with Two-dimensional Metamaterialbased Passive Phased Array," Scientific Reports, vol. 6, p. 35437, dec 2016.

[13] H. Estrada, A. Uris, and F. Meseguer, "Acoustic radiation efficiency of a periodically corrugated rigid piston," Applied Physics Letters, vol. 101, p. 104103, sep 2012.

[14] M. Martins, V. Correia, J. Cabral, S. Lanceros-Mendez, and J. Rocha, "Optimization of piezoelectric ultrasound emitter transducers for underwater communications," Sensors and Actuators A: Physical, vol. 184, pp. 141-148, sep 2012.

[15] T.-D. Luong, T. Hies, and C.-D. Ohl, "A compact time reversal emitterreceiver based on a leaky random cavity," Scientific Reports, vol. 6, p. 36096, dec 2016.

[16] D. R. Smith, V. R. Gowda, O. Yurduseven, S. Larouche, G. Lipworth, Y. Urzhumov, and M. S. Reynolds, "An analysis of beamed wireless power transfer in the Fresnel zone using a dynamic, metasurface aperture," Journal of Applied Physics, vol. 121, p. 014901, jan 2017. 
Chapter 3. On the use of Phase Correction Rings on Fresnel Zone Plates with Ultrasound Piston Emitters 


\section{Chapter 4}

\section{Bifocal Ultrasound Focusing using Bi-Fresnel Zone Plate Lenses}

S. Pérez-López, J. M. Fuster, P. Candelas, D. Tarrazó-Serrano,

S. Castiñeira-lbáñez, and C. Rubio, "Bifocal Ultrasound Focusing Using Bi-Fresnel Zone Plate Lenses," Sensors, vol. 20, no. 3, p. 705, Jan. 2020.

https://doi.org/10.3390/s20030705

\section{Abstract}

In this work, we present a bifocal Fresnel zone plate (BiFZP) capable of generating focusing profiles with two different foci. The performance of the BiFZP is demonstrated in the ultrasound domain, with very good agreement between experimental measurements and FEM simulations. This lens becomes an appealing alternative to other dual-focusing lenses, in which the foci location can only be set to a limited range of positions, such as M-bonacci zone plates. Moreover, the variation of the operating frequency has also been analyzed, providing an additional dynamic control parameter in this type of lenses. 


\subsection{Introduction}

Acoustic focusing is a hot topic among the scientific community due to its multiple applications in both industrial and healthcare sectors. In the past years, several techniques to focus acoustic waves have been reported, such as time-reversal mirrors, metasurfaces or holographic lenses. Time-reversal mirrors (TRMs) take advantage of the time-reversal invariance of the wave equation to produce a focal spot at the target over an inhomogeneous acoustic medium [1-4]. On the other hand, acoustic metasurfaces employ 3D unit cells to generate a phase distribution at the output of the lens that ensures constructive interference at the desired focal length [5-8]. Another interesting approach to focus ultrasonic beams are holographic lenses. Holographic lenses employ back-propagation techniques and iterative algorithms to produce an arbitrarily-shaped focused beam over a specific focal area [9-12]. However, all these techniques have some drawbacks which may limit their application or their design. For instance, TRMs often require expensive phase-arrays to produce a narrow focal spot, acoustic metasurfaces need a complete 3D design of each one of their unit cells, and holographic lenses require a high computation burden to design via an iterative back-propagation process the 3D height profile of the lens. In this sense, Fresnel Zone Plates (FZPs) are a more conventional yet effective approach to achieve ultrasound focusing with high resolution.

FZPs are monofocal planar lenses formed by concentric rings of decreasing width known as Fresnel regions. These devices have been widely used in various fields of physics, such as X-rays [13, 14], optics [15-17], microwaves [18, 19] or acoustics [20-22]. In [23], J. Kim et al. presented a method to achieve multifocal focusing profiles based on the phase conjugation principle and the phase-opposition blocking criterion used in conventional FZPs. Recently, novel lenses based on traditional FZPs have been introduced in optics [24-27] and later demonstrated in acoustics [28-30], which increases the versatility of these devices. Among these novel designs, M-bonacci Zone Plates (MbZPs) stand out for their interesting bifocal focusing profiles [26, 27], and can produce two foci with equal acoustic intensities. However, MbZPs are only capable of generating foci at specific focal distances related with the M-bonacci sequence ratio, which significantly limits their flexibility [27]. The ratio between both foci in a MbZP remains constant when the order of the M-bonacci sequence is selected. As an example, in a Fibonacci ZP (2-order MbZP), the foci ratio is 1.618 and cannot be modified. Similarly, foci ratios of 1.192 and 1.078 are obtained when using MbZPs of order 3 and 4, respectively. This work demonstrates a composite bifocal FZP, hereafter referred to as BiFZP, capable of producing a focusing profile with two foci. This BiFZP can be used in scenarios where MbZPs cannot achieve the required focal distances, and therefore it represents an interesting and flexible alternative to this kind of 
lenses.

\subsection{BiFZP Lens Design}

For plane wave incidence, the radii of the different Fresnel regions that form a FZP can be calculated as

$$
r_{n}=\sqrt{n \lambda F+\left(\frac{n \lambda}{2}\right)^{2}}
$$

where $F$ is the focal length, $\lambda$ is the working wavelength and $n=1,2, \ldots, N$, being $N$ the total number of Fresnel regions.

Once the FZP radii are calculated, the focusing profile of the lens is obtained by numerically computing the Rayleigh-Sommerfeld diffraction integral,

$$
I(z)=\frac{4 \pi^{2}}{\lambda^{2}}\left|\int_{0}^{r_{N}} p_{i}\left(r^{\prime}\right) t\left(r^{\prime}\right) \frac{e^{-j k R}}{R} \cos (n, R) r^{\prime} d r^{\prime}\right|^{2},
$$

where $r_{N}$ is the maximum radius of the lens, $r^{\prime}$ is the radial coordinate of the lens, $k=2 \pi / \lambda$ is the wavenumber, $p_{i}\left(r^{\prime}\right)$ is the incident pressure distribution, $t\left(r^{\prime}\right)$ is the ZP transmittance function, $R=\sqrt{\left(r^{\prime}\right)^{2}+z^{2}}, z$ is the axial coordinate and $\cos (n, R)=z / R$, being $n$ the normal direction to the lens surface. For a Soret $\mathrm{ZP}$, the transmittance function, also known as pupil function, is 0 at the pressure blocking regions and 1 at the transparent regions. 
(a)
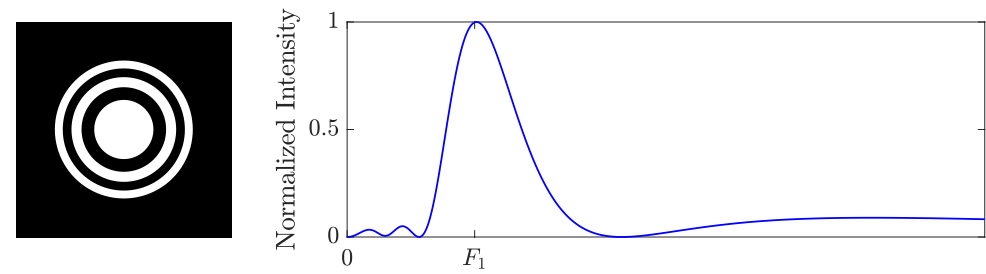

(b)
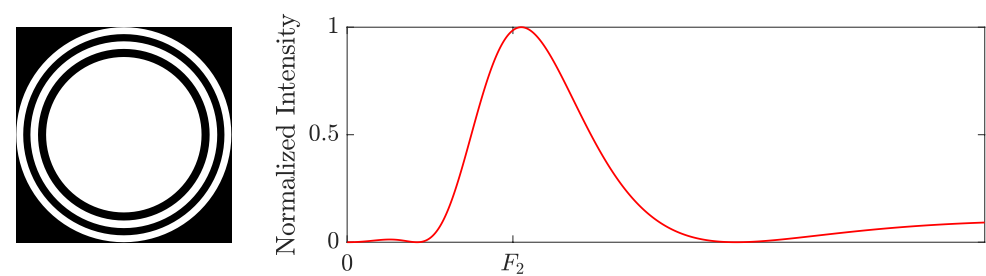

(c)

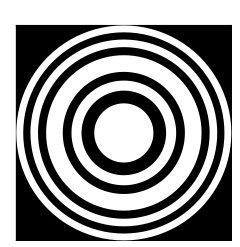

$r^{\prime}(\mathrm{m})$

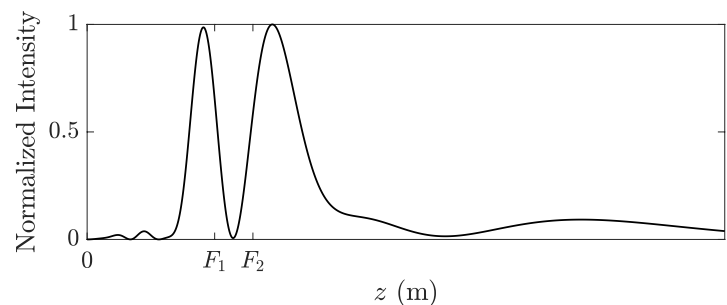

Figure 4.1: FZP layouts and their corresponding normalized focusing profiles: (a) Inner FZP, (b) outer FZP, and (c) BiFZP.

The BiFZP lens is obtained by combining Fresnel regions of two conventional FZPs. The inner regions are designed to focus at a focal length $F_{1}$, while the outer regions are designed to focus at a different focal length $F_{2}$. In this process, $F_{1}$ is selected to be closer to the lens, that is, $F_{1}<F_{2}$, which provides a better focusing performance. Figure 4.1 shows a schematic example of the lens design steps. Figure 4.1(a) depicts the layout of the inner Fresnel regions that have been designed using Equation 4.1 to focus at $F_{1}$ (left), and its corresponding focusing profile (right); while Figure 4.1(b) depicts the layout of the outer regions that focus at $F_{2}$ (left) and its corresponding focusing profile (right). Here, each focusing profile is normalized to its maximum value. As can be observed from the figure, the focal length in this focusing profile, which theoretically corresponds to $F_{2}$, presents a slight shift due to the pupil effect of the layout depicted in Figure 4.1(b) (right), which has already been analyzed in [31].

Figure 4.1(c) (left) depicts the BiFZP layout, obtained by combining the inner and outer regions from the layouts of Figure 4.1(a) and 4.1(b). As can be 
observed from Figure 4.1(c) (right), the focusing profile of the BiFZP achieves two isolated foci, which are encountered at the vicinity of their expected locations $\left(F_{1}\right.$ and $F_{2}$ ). Both BiFZP foci present higher longitudinal resolution than those from their inner and outer FZP counterparts. Moreover, the focal lengths of the BiFZP foci are slightly shifted from their theoretical values $F_{1}$ and $F_{2}$. This fact is a consequence of the interference between the phase profiles of the inner and outer Fresnel regions that form the lens. In order to showcase this phenomenon, Figure 4.2 depicts the normalized focusing profile of a BiFZP alongside with an inset of the phase profiles of the inner (blue color) and outer (red color) regions. The lens has been designed for focal distances $F_{1}=50 \mathrm{~mm}, F_{2}=65 \mathrm{~mm}$, frequency $f=337.5 \mathrm{kHz}$ and a total number of Fresnel regions of $N=9$. The focusing profile has been computed using the Rayleigh-Sommerfeld diffraction integral. As can be observed from the figure, both foci are shifted towards the areas where the inner and outer phase profiles are in-phase $\left(F_{1}^{\prime}=45.6 \mathrm{~mm}\right.$ and $\left.F_{2}^{\prime}=72.6 \mathrm{~mm}\right)$, and the zero intensity value at $z \cong 57 \mathrm{~mm}$ is achieved because both profiles are in phase opposition. Therefore, the BiFZP has to be designed considering the phase profiles of the inner and outer regions of the lens, as they play a very important role on the final focusing performance of the device.

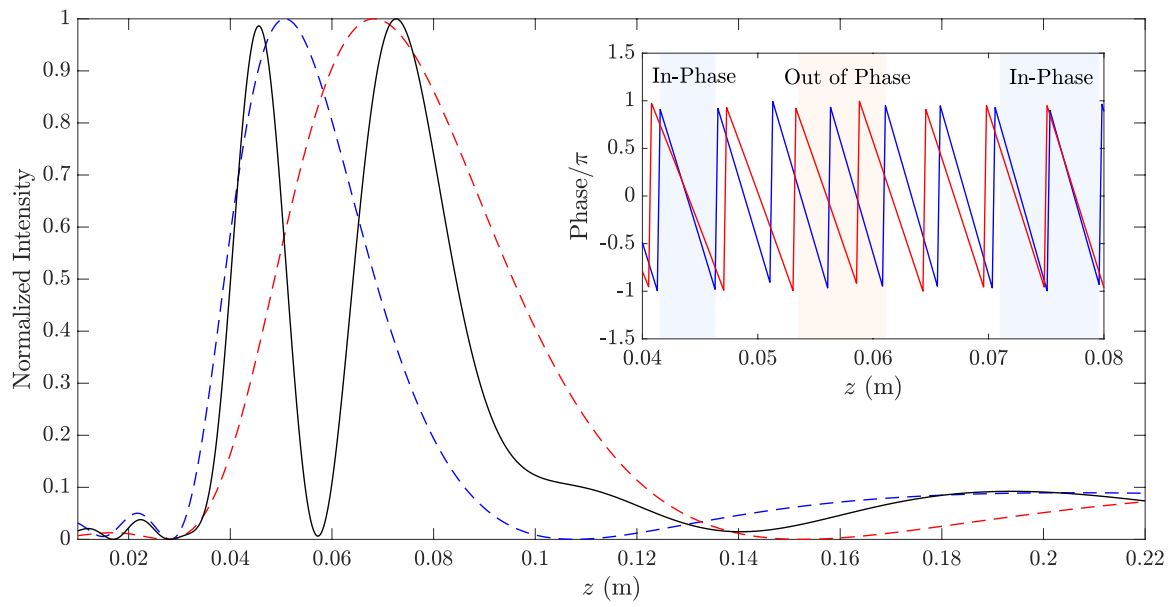

Figure 4.2: Bi-Fresnel Zone Plate focusing profile (black) compared to its inner (blue) and outer (red) focusing profiles. The phase of the inner (blue) and outer (red) profiles are shown at the inset.

In order to showcase the flexibility of the proposed design method, Figure 4.3 depicts two additional focusing profiles obtained using two different BiFZPs at $250 \mathrm{kHz}$. The BiFZP focusing profile depicted in Figure 4.3(a) (black line) is designed to focus at $F_{1}^{\prime}=40 \mathrm{~mm}$ and $F_{2}^{\prime}=68 \mathrm{~mm}$. The monofocal FZPs 
that have been combined to build this BiFZP focus at $F_{1}=44.4 \mathrm{~mm}$ (blue line) and $F_{2}=59.5 \mathrm{~mm}$ (red line), respectively. Using a different BiFZP, Figure 4.3(b) shows a focusing profile (black line) with two foci located at $F_{1}^{\prime}=56 \mathrm{~mm}$ and $F_{2}^{\prime}=90 \mathrm{~mm}$. The two corresponding monofocal FZPs in this case focus at $F_{1}=60.2 \mathrm{~mm}$ (blue line) and $F_{2}=80.1 \mathrm{~mm}$ (red line), respectively. As can be observed from Figure 4.3, both lenses achieve their expected design focal distances, showing, as a consequence of the phase profile interference, two welldefined foci with similar intensities and a sharp minimum very close to zero which clearly isolates both foci.

(a)

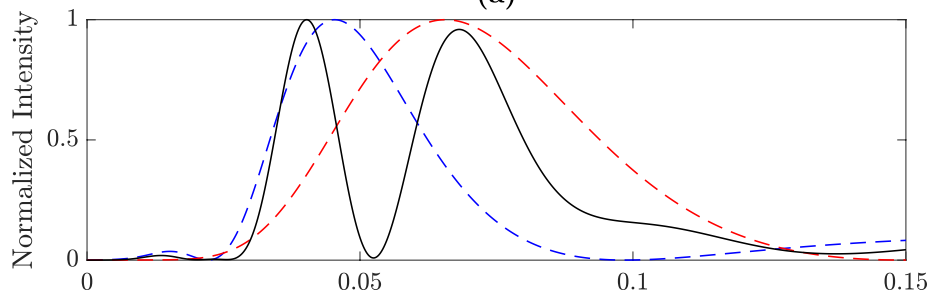

(b)

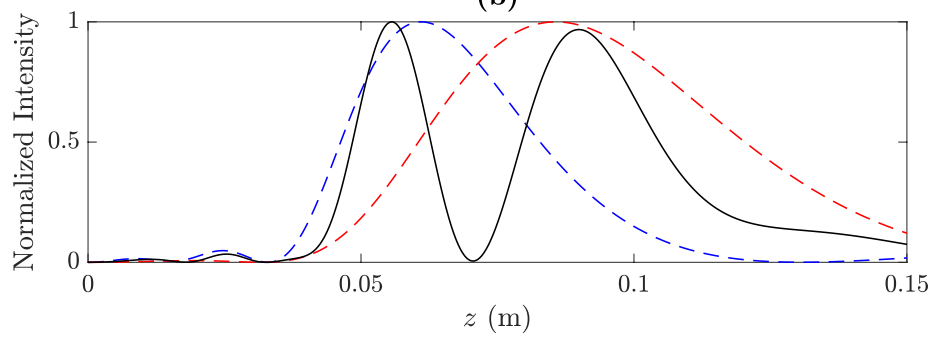

Figure 4.3: (a) BiFZP focusing profile designed for $F_{1}^{\prime}=40 \mathrm{~mm}$ and $F_{2}^{\prime}=68$ $\mathrm{mm}$, and (b) BiFZP focusing profile designed for $F_{1}^{\prime}=56 \mathrm{~mm}$ and $F_{2}^{\prime}=90 \mathrm{~mm}$. In both cases, $f_{0}=250 \mathrm{kHz}$.

In order to validate the design method, experimental measurements with a brass BiFZP have been carried out. As in the experimental set-up the distance between transducer and lens is not large enough to consider plane wave incidence, the Fresnel radii have been calculated considering spherical wave incidence [32], that is,

$$
d+F+\frac{n \lambda}{2}=\sqrt{d^{2}+r_{n}^{2}}+\sqrt{F^{2}+r_{n}^{2}}
$$

where $d$ is the distance between transducer and lens. 
The final design of the lens is obtained using a two-step process. At the first step, ideal simulations are carried out using MATLAB to numerically calculate the Rayleigh-Sommerfeld integral (Equation 4.2), in order to obtain the focal lengths so that the resulting focusing profile exhibits two well-defined foci with an intermediate zero value. Once $F_{1}$ and $F_{2}$ are obtained for the design frequency, iterative Finite Element Method (FEM) simulations are carried out using COMSOL Multiphysics, in order to optimize the number of inner and outer Fresnel regions that provide a focusing profile with balanced intensities at both foci. FEM simulations solve the wave equation in each medium (water and brass), taking into account acoustic solid-liquid interactions, and therefore providing a better characterization of the lens performance. It is worth noting that the number of inner and outer Fresnel regions can only be increased or decreased in discrete steps, which means that the focal intensities will also increase or decrease in discrete levels.

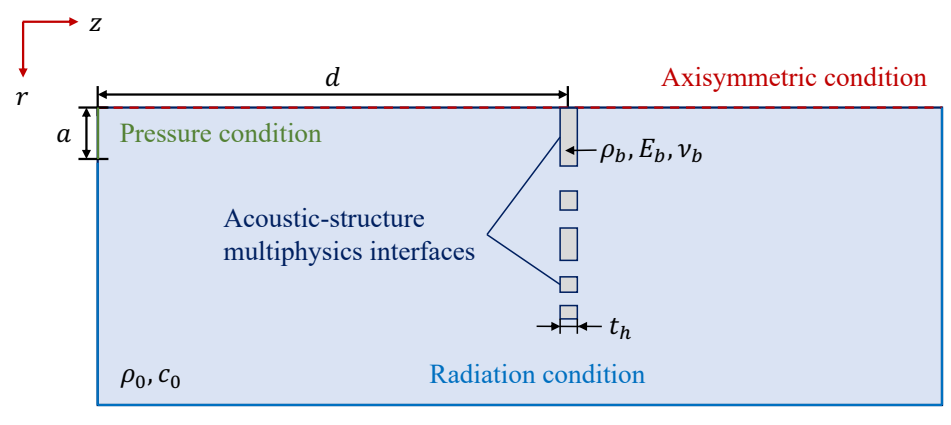

Figure 4.4: Scheme of the Finite Element Method (FEM) model.

Figure 4.4 shows a scheme of the axisymmetric FEM model employed in the simulation. The lens is modelled as a linear elastic material immersed in water, with $t_{h}$ being the thickness of the lens. An acoustic-structure multiphysics interface is established at the lens boundaries in order to account for the solidliquid wave interactions. The transducer is modelled as a pressure condition of length $a$, being $a$ the active radius of the transducer, separated a distance $d$ from the lens, and a radiation condition is set at the outer boundaries in order to avoid reflections. Table 4.1 shows the acoustic properties of the materials used in the simulations. In this table, $\rho$ represents the material density, $E$ the Young modulus, $\nu$ the Poisson's ratio, and $c$ the speed of sound. 


\begin{tabular}{ll}
\hline Material & Property \\
\hline \multirow{3}{*}{ Brass } & $\rho_{b}=8500 \mathrm{~kg} / \mathrm{m}^{3}$ \\
& $E_{b}=104 \mathrm{GPa}$ \\
& $\nu_{b}=0.37$ \\
\hline \multirow{2}{*}{ Water } & $\rho_{0}=1000 \mathrm{~kg} / \mathrm{m}^{3}$ \\
& $c_{0}=1500 \mathrm{~m} / \mathrm{s}$ \\
\hline
\end{tabular}

Table 4.1: Acoustic properties used in FEM simulations.

\subsection{Experimental Results and Discussion}

The experimental set-up consists of a 3D underwater automated positioning system with a spatial resolution of $1 \times 1 \times 1 \mathrm{~mm}^{3}$. An Imasonic piston transducer with $30 \mathrm{~mm}$ of active diameter and a central frequency of $250 \mathrm{kHz}$ is used as emitter. The feeding signal of the transducer is generated using a Panametrics 5077PR pulser.

(a)

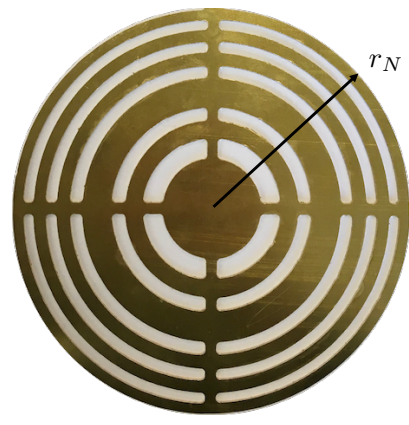

(b)

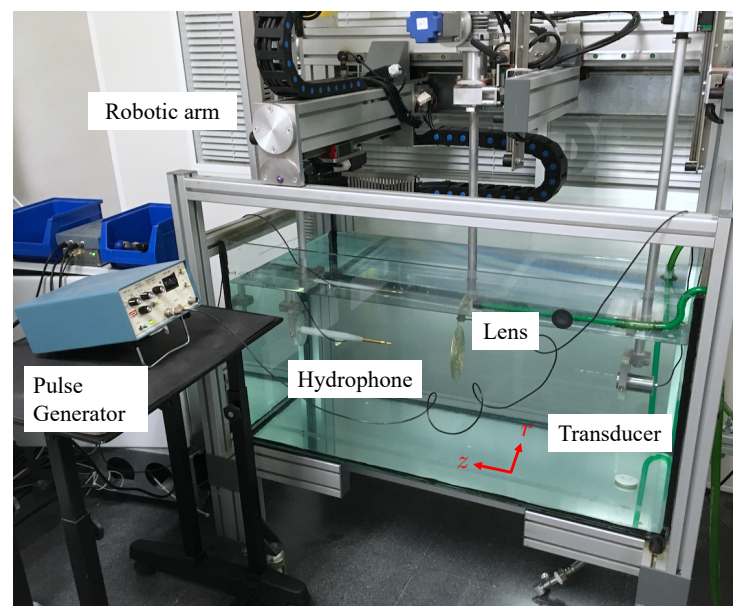

Figure 4.5: (a) Manufactured BiFZP lens made of brass and (b) experimental set-up with the lens.

The receiver consists of a needle hydrophone from Precision Acoustics Ltd. with $1 \mathrm{~mm}$ of active diameter and a $-4 \mathrm{~dB}$ bandwidth ranging from $200 \mathrm{kHz}$ to 25 $\mathrm{MHz}$. The needle hydrophone is connected to a low noise preamplifier and then to a digital oscilloscope from Pico Technology. The manufactured BiFZP is made of brass with a thickness of $t_{h}=1 \mathrm{~mm}$ and is located at a distance $d=350$ 
$\mathrm{mm}$ from the piston transducer. The lens has been designed for focal lengths $F_{1}=50 \mathrm{~mm}$ and $F_{2}=70 \mathrm{~mm}$, and a design frequency of $250 \mathrm{kHz}$. The BiFZP outermost radius is $r_{N}=66.71 \mathrm{~mm}$. Figures $4.5(\mathrm{a})$ and $4.5(\mathrm{~b})$ show a picture of the manufactured brass BiFZP and the experimental set-up, respectively.

Figures 4.6(a) and 4.6(b) depict the simulated and measured acoustic intensity maps, respectively. Each map is normalized to its maximum value. As can be observed, FEM and experimental results are in good agreement, and the manufactured BiFZP provides a bifocal focusing profile with two well-defined foci.
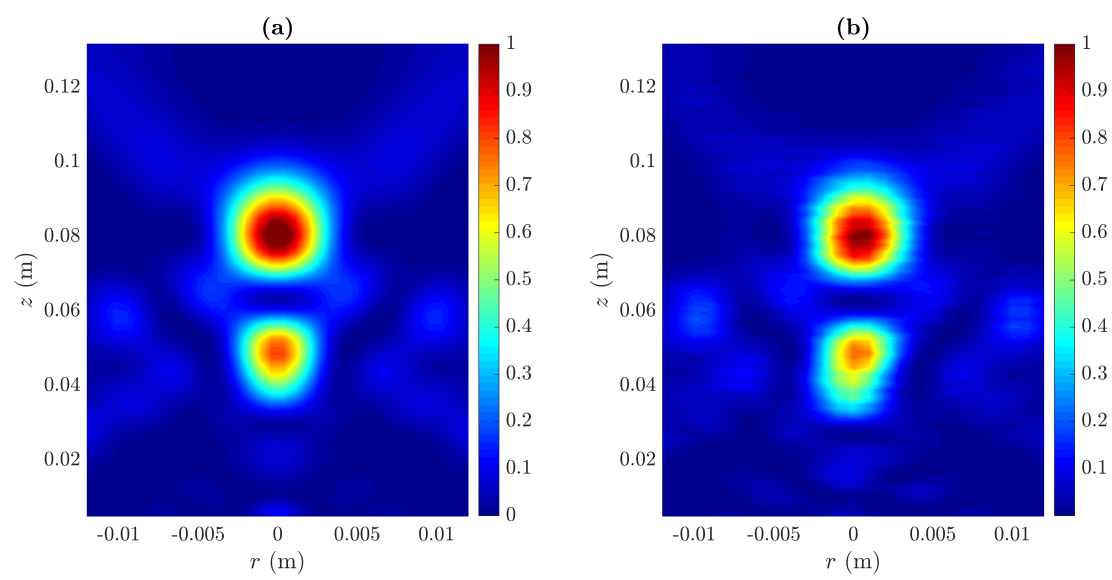

Figure 4.6: (a) Simulated and (b) measured normalized acoustic intensity maps for the manufactured BiFZP lens.

Figure 4.7 shows the longitudinal and transversal cuts of the acoustic intensity maps shown in Figure 4.6. Experimental measurements are represented using black squares, whereas simulated results are depicted as solid lines in both longitudinal (blue) and transversal (red) cuts.

The longitudinal cut shown in Figure 4.7(a) corresponds to the BiFZP focusing profile at $r=0$. As expected, the focal lengths are shifted from the theoretical values $\left(F_{1}=50 \mathrm{~mm}\right.$ and $\left.F_{2}=70 \mathrm{~mm}\right)$ to $F_{1}^{\prime}=47.8 \mathrm{~mm}$ and $F_{2}^{\prime}=79.8 \mathrm{~mm}$, as a consequence of the interference between the inner and outer phase profiles. As can be observed from the figure, both foci are clearly isolated and a very well-defined null can be found at $z=62.8 \mathrm{~mm}$.

The transversal cuts corresponding to $F_{1}^{\prime}$ and $F_{2}^{\prime}$ are shown in Figures 4.7(b) and 4.7(c), respectively. As can be observed, there is a slight decrease in lateral resolution at the second focus $\left(F_{2}\right)$, due to the fact that it is located further apart from the BiFZP. This effect becomes more noticeable when the 
(a)

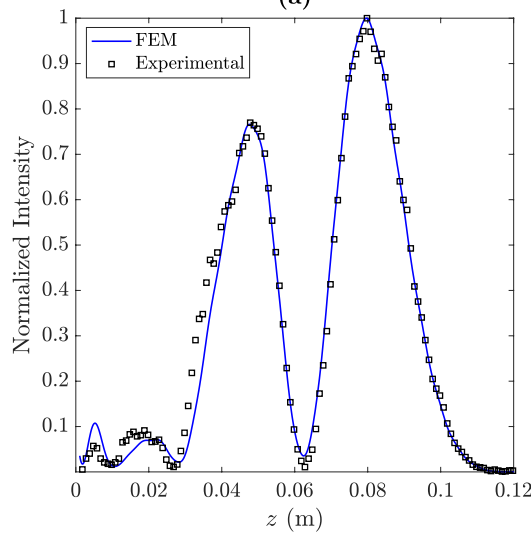

(b)

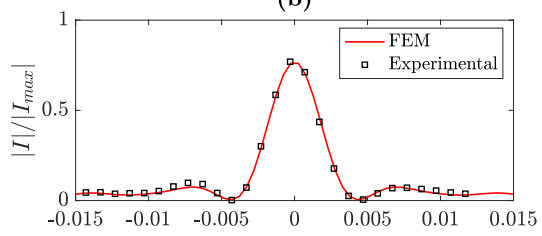

(c)

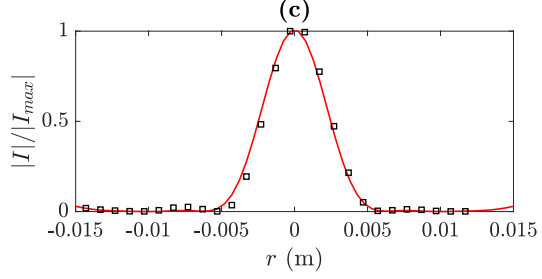

Figure 4.7: (a) Measured (black squares) and simulated (blue line) focusing profiles (longitudinal cuts) for the manufactured BiFZP lens. (b,c) Measured (black squares) and simulated (red lines) transversal cuts at $z=F_{1}^{\prime}$ (b) and $z=F_{2}^{\prime}$ (c).

distance between the two foci of the BiFZP lens is further increased. As can be observed from Figure 4.7, experimental measurements are in good agreement with simulation results.

Figure 4.8(a) shows the focal length dependence on the operating frequency. As can be observed from the figure, both focal lengths shift linearly with frequency, which agrees with the results reported in previous works [33, 29]. Moreover, Figure 4.8(b) depicts the simulated and measured focusing profiles for three different frequencies: $220 \mathrm{kHz}$ (top), $250 \mathrm{kHz}$ (middle), and $280 \mathrm{kHz}$ (bottom). Thus, when the operating frequency is increased both foci are shifted away from the lens, and therefore the operating frequency becomes an interesting parameter to dynamically control the focusing profile of the lens.

\subsection{Conclusions}

In this work, a design method to obtain bifocal focusing profiles by combining two conventional monofocal FZPs is reported. Experimental results are presented, showing good agreement with numerical simulations and demonstrating that BiFZPs can become an appealing alternative to MbZPs in scenarios where MbZPs cannot achieve the required focal lengths. In addition, BiFZPs present a linear dependence on the operating frequency, which can become an interesting 
(a)

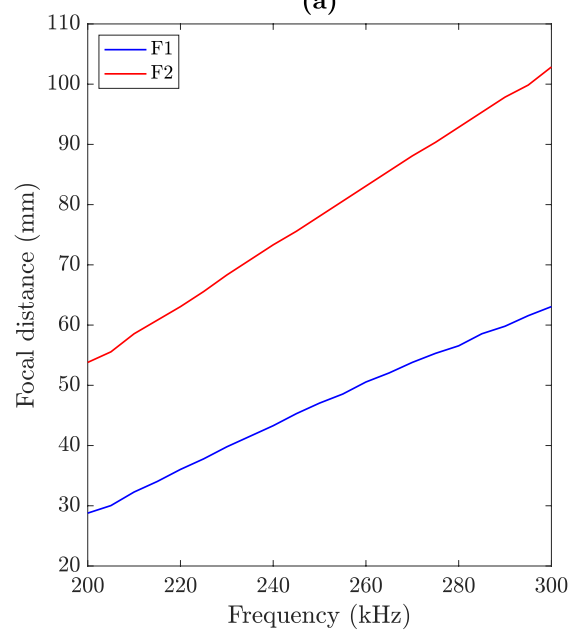

(b)
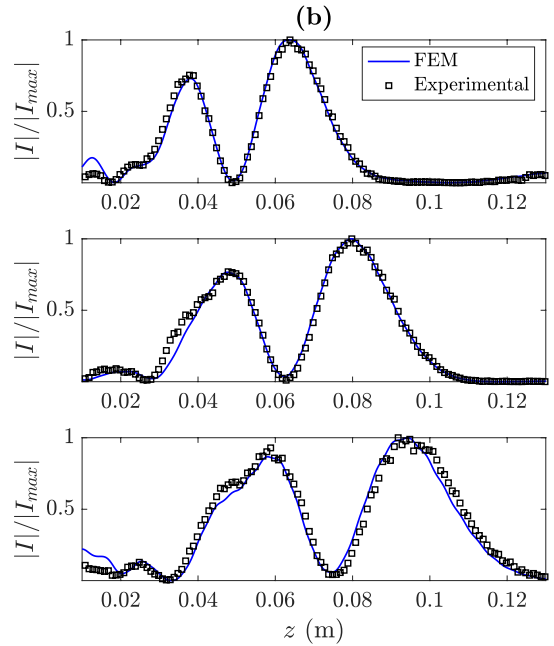

Figure 4.8: (a) BiFZP focal distance for $F_{1}^{\prime}$ (blue) and $F_{2}^{\prime}$ (red) as a function of the operating frequency and (b) BiFZP focusing profiles at $220 \mathrm{kHZ}$ (top), 250 $\mathrm{kHz}$ (middle) and $280 \mathrm{kHz}$ (bottom).

parameter to dynamically tune the focal lengths once the lens is manufactured.

\section{References}

[1] M. Fink, "Time reversal of ultrasonic fields. I. Basic principles," IEEE Transactions on Ultrasonics, Ferroelectrics and Frequency Control, vol. 39, pp. 555566, sep 1992.

[2] M. Fink, D. Cassereau, A. Derode, C. Prada, P. Roux, M. Tanter, J.-L. Thomas, and F. Wu, "Time-reversed acoustics," Reports on Progress in Physics, vol. 63, pp. 1933-1995, dec 2000.

[3] Y. Jing, F. C. Meral, and G. T. Clement, "Time-reversal transcranial ultrasound beam focusing using a k-space method," Physics in Medicine and Biology, vol. 57, pp. 901-917, feb 2012.

[4] J. L. B. Robertson, B. T. Cox, J. Jaros, and B. E. Treeby, “Accurate simulation of transcranial ultrasound propagation for ultrasonic neuromodulation and stimulation," The Journal of the Acoustical Society of America, vol. 141, pp. 1726-1738, mar 2017. 
[5] Y. Li, B. Liang, X. Tao, X.-f. Zhu, X.-y. Zou, and J.-c. Cheng, "Acoustic focusing by coiling up space," Applied Physics Letters, vol. 101, p. 233508, dec 2012.

[6] Y. Xie, W. Wang, H. Chen, A. Konneker, B.-I. Popa, and S. A. Cummer, "Wavefront modulation and subwavelength diffractive acoustics with an acoustic metasurface," Nature Communications, vol. 5, p. 5553, dec 2014.

[7] B. Assouar, B. Liang, Y. Wu, Y. Li, J. C. Cheng, and Y. Jing, "Acoustic metasurfaces," Nature Reviews Materials, vol. 3, pp. 460-472, dec 2018.

[8] J. Chen, J. Xiao, D. Lisevych, A. Shakouri, and Z. Fan, "Deep-subwavelength control of acoustic waves in an ultra-compact metasurface lens," Nature Communications, vol. 9, p. 4920, dec 2018.

[9] R. Lalonde, A. Worthington, and J. Hunt, "Field conjugate acoustic lenses for ultrasound hyperthermia," IEEE Transactions on Ultrasonics, Ferroelectrics and Frequency Control, vol. 40, pp. 592-602, sep 1993.

[10] K. Melde, A. G. Mark, T. Qiu, and P. Fischer, "Holograms for acoustics," Nature, vol. 537, pp. 518-522, sep 2016.

[11] S. Jiménez-Gambín, N. Jiménez, J. M. Benlloch, and F. Camarena, "Holograms to Focus Arbitrary Ultrasonic Fields through the Skull," Physical Review Applied, vol. 12, p. 014016, jul 2019.

[12] M. D. Brown, "Phase and amplitude modulation with acoustic holograms," Applied Physics Letters, vol. 115, p. 053701, jul 2019.

[13] J. Kirz, "Phase zone plates for $x$ rays and the extreme uv," Journal of the Optical Society of America, vol. 64, p. 301, mar 1974.

[14] K. Jefimovs, O. Bunk, F. Pfeiffer, D. Grolimund, J. van der Veen, and C. David, "Fabrication of Fresnel zone plates for hard X-rays," Microelectronic Engineering, vol. 84, pp. 1467-1470, may 2007.

[15] P. Srisungsitthisunti, O. K. Ersoy, and X. Xu, "Laser direct writing of volume modified Fresnel zone plates," Journal of the Optical Society of America B, vol. 24, p. 2090, sep 2007.

[16] R. S. Rodrigues Ribeiro, P. Dahal, A. Guerreiro, P. A. S. Jorge, and J. Viegas, "Fabrication of Fresnel plates on optical fibres by FIB milling for optical trapping, manipulation and detection of single cells," Scientific Reports, vol. 7, p. 4485, dec 2017. 
[17] H. Kim, J. Kim, H. An, Y. Lee, G.-y. Lee, J. Na, K. Park, S. Lee, S.-Y. Lee, B. Lee, and $Y$. Jeong, "Metallic Fresnel zone plate implemented on an optical fiber facet for super-variable focusing of light," Optics Express, vol. 25, p. 30290, nov 2017.

[18] H. Hristov and M. Herben, "Millimeter-wave Fresnel-zone plate lens and antenna," IEEE Transactions on Microwave Theory and Techniques, vol. 43, no. 12, pp. 2779-2785, 1995.

[19] H. D. Hristov and J. M. Rodriguez, "Design Equation for Multidielectric Fresnel Zone Plate Lens," IEEE Microwave and Wireless Components Letters, vol. 22, pp. 574-576, nov 2012.

[20] S. A. Farnow and B. A. Auld, "An Acoustic Phase Plate Imaging Device," in Acoustical Holography, pp. 259-273, Boston, MA: Springer US, 1975.

[21] M. Z. Sleva, W. D. Hunt, and R. D. Briggs, "Focusing performance of epoxyand air-backed polyvinylidene fluoride Fresnel zone plates," The Journal of the Acoustical Society of America, vol. 96, pp. 1627-1633, sep 1994.

[22] D. C. Calvo, A. L. Thangawng, M. Nicholas, and C. N. Layman, "Thin Fresnel zone plate lenses for focusing underwater sound," Applied Physics Letters, vol. 107, p. 014103, jul 2015.

[23] J. Kim, H. Kim, G.-Y. Lee, J. Kim, B. Lee, and Y. Jeong, "Numerical and Experimental Study on Multi-Focal Metallic Fresnel Zone Plates Designed by the Phase Selection Rule via Virtual Point Sources," Applied Sciences, vol. 8, p. 449, mar 2018.

[24] G. Saavedra, W. D. Furlan, and J. A. Monsoriu, "Fractal zone plates," Optics Letters, vol. 28, p. 971, jun 2003.

[25] W. D. Furlan, G. Saavedra, and J. A. Monsoriu, "White-light imaging with fractal zone plates," Optics Letters, vol. 32, no. 15, p. 2109, 2007.

[26] J. A. Monsoriu, A. Calatayud, L. Remon, W. D. Furlan, G. Saavedra, and P. Andres, "Bifocal Fibonacci Diffractive Lenses," IEEE Photonics Journal, vol. 5, pp. 3400106-3400106, jun 2013.

[27] F. Machado, V. Ferrando, W. D. Furlan, and J. A. Monsoriu, "Diffractive m-bonacci lenses," Optics Express, vol. 25, p. 8267, apr 2017.

[28] J. Fuster, S. Pérez-López, P. Candelas, and C. Rubio, "Design of BinarySequence Zone Plates in High Wavelength Domains," Sensors, vol. 18, p. 2604, aug 2018. 
[29] S. Pérez-López, J. M. Fuster, P. Candelas, and C. Rubio, "Fractal lenses based on Cantor binary sequences for ultrasound focusing applications," Ultrasonics, vol. 99, p. 105967, nov 2019.

[30] S. Pérez-López, J. M. Fuster, and P. Candelas, "M-Bonacci Zone Plates for Ultrasound Focusing," Sensors, vol. 19, p. 4313, oct 2019.

[31] S. Castiñeira-Ibáñez, D. Tarrazó-Serrano, O. V. Minin, C. Rubio, and I. V. Minin, "Tunable depth of focus of acoustical pupil masked Soret Zone Plate," Sensors and Actuators A: Physical, vol. 286, pp. 183-187, feb 2019.

[32] S. Pérez-López, J. M. Fuster, P. Candelas, C. Rubio, and F. Belmar, "On the use of phase correction rings on Fresnel zone plates with ultrasound piston emitters," Applied Physics Letters, vol. 112, p. 264102, jun 2018.

[33] J. M. Fuster, P. Candelas, S. Castiñeira-lbáñez, S. Pérez-López, and C. Rubio, "Analysis of fresnel zone plates focusing dependence on operating frequency," Sensors (Switzerland), vol. 17, p. 2809, dec 2017. 


\title{
Chapter 5
}

\section{Transient Analysis of Fresnel Zone Plates for Ultrasound Focusing Applications}

\author{
S. Pérez-López, D. Tarrazó-Serrano, D. O. Dolmatov, C. Rubio, \\ and P. Candelas, "Transient Analysis of Fresnel Zone Plates for \\ Ultrasound Focusing Applications," Sensors, vol. 20, no. 23, p. \\ 6824, Nov. 2020. \\ https://doi.org/10.3390/s20236824
}

\section{Abstract}

Fresnel Zone Plates are planar lenses that can be used to focus ultrasound beams. This kind of acoustic lenses can play a key role in the resolution of ultrasonic NDT systems. In this type of pulse-echo applications, the pulse duration is an important parameter that specifies the axial resolution, and thus, shorter ultrasound pulses provide higher resolutions. However, acoustic lenses exhibit a transient response that should be considered when setting the pulse duration, as pulses shorter than the transient state duration result in degradation in the response of acoustic lenses in terms of focal intensity, focal displacement, and lateral and axial resolutions. In this work, a thorough analysis of the transient response of Fresnel Zone Plates is discussed, demonstrating that the transient state should be considered in order 
to achieve optimal focusing performance. Theoretical and numerical results are presented, showing very good agreement.

\subsection{Introduction}

The generation of focused acoustic fields is a hot topic in different scientific and engineering fields, including pharmaceutical, sonochemistry, and non-destructive testing [1-3]. In the past years, different novel approaches to focus acoustic beams were proposed, including the application of metasurfaces [4], time-reversal mirrors [5], holographic lenses [6], Polyadic Cantor Fractal lenses [7], Pinhole and Fresnel Zone Plates [8, 9]. Fresnel Zone Plates (FZPs) are a very appealing option to focus acoustic waves in cases where the size, weight, and production simplicity of the acoustic lens are key constraints. FZPs are planar lenses with an easy design and manufacturing process, and can be effectively employed in both air and underwater applications [10, 11]. FZPs consist of a series of concentric Fresnel regions. The phase difference from the lens to the focus between two consecutive regions is $\pi$, which means that the pressure contributions between consecutive regions interfere destructively at the focus. Based on this phenomenon, FZP lenses can be classified in two types, depending on how this phase change is treated: Soret FZPs and Phase-Reversal FZPs [12].

Flaw detection and sizing is one of the main applications of pulse-echo ultrasonic testing, which makes the task of focusing acoustic fields relevant. With the aim of improving the resolution of these techniques, J. Salazar et al. carried out various studies. They proposed the application of two pulses to excite the transducer (the so-called pulse cancellation technique [13]). Moreover, the same authors discussed an air-coupled ultrasonic testing system based on the pulse cancellation technique [14]. In general, acoustic focusing is obtained using transducers with concave surfaces and testing objects immersed in water. The application of FZPs has the potential to increase the versatility of ultrasonic inspections. This is related to the fact that the parameters of the focal spot (depth, length, and width) can be modified via the appropriate design of the FZP according to the objectives and conditions of the testing.

One of the basic features of pulse-echo testing is the application of pulsed ultrasonic signals. In this sense, the signal duration should be short enough to provide results with the required axial resolution. Previous studies showed that pulse compression techniques can be used to decrease the ultrasound pulse length in order to improve the axial resolution [15]. Short impulses allow to effectively resolve the targets of pulse-echo ultrasonic non-destructive testing. It is possible 
to reduce the size of the dead zone, increase the resolution and precision in determining the distance to the reflector for testing objects [16]. On the other hand, using acoustic lenses requires employing excitation signals with shapes and durations that result in an effective focusing performance. In this regard, introducing FZPs to the pulse-echo ultrasonic testing field requires a comprehensive study on how the shape and duration of the pulsed signals affects the focusing efficiency of FZP lenses.

In this work, a thorough analysis of the transient state of FZP lenses is presented. Three waveform types have been considered, including Continuous Wave (CW), Modulated Rectangular Pulse (MRP), and Modulated Gaussian Pulse (MGP). The pulse duration influence on the main lens parameters is studied, demonstrating that if the pulse length is shorter than the transient state duration of the lens, optimal resolution parameters are not achieved.

\subsection{Results}

\subsubsection{FZP Transient Duration}

FZP lenses are widely used in many application fields due to their simple design, planar fabrication, and good focusing performance. They are made of circular concentric rings with decreasing width, known as Fresnel regions. Each Fresnel region is in phase-opposition with the previous one, meaning that it generates a destructive wave interference at the focal distance of the lens. Thus, FZPs can be divided into two types, depending on how they handle the phase-opposition regions. Soret FZPs block phase-opposition regions with pressure opaque rings so the resulting lens is an alternating sequence of transparent and pressure blocking rings. On the other hand, Rayleigh-Wood FZPs, also known in literature as Phase Zone Plates or Phase-Reversal FZPs, replace the blocking regions with phasereversal rings, which introduce a $\pi$-phase shift and therefore all the Fresnel regions of the lens can contribute constructively at the focus.

The design condition of the lens is that the propagation path difference from the radius of each contiguous region to the focus has to be $\lambda / 2$, which provides the phase-opposition condition between consecutive Fresnel regions. Thus, if plane wave incidence is considered, the radius of each Fresnel region, $r_{n}$, is given by

$$
r_{n}=\sqrt{n \lambda F+\left(\frac{n \lambda}{2}\right)^{2}}
$$


where $\lambda=c / f$ is the wavelength, being $c$ the sound speed in the medium and $f$ the working frequency, $F$ is the focal distance, and $n=1,2, \ldots, N$, where $N$ is the total number of Fresnel regions.

When a plane wavefront hits the lens, the time of flight from each region to the focus is different, as propagation paths of outer rings are longer than those of inner rings. This means that the lens will have a transient response, starting with the arrival of the wavefront generated from the diffraction at the first Fresnel region, and ending with the arrival of the wavefront diffracted from the last region of the lens. After this transient response, a steady state is reached, as the focus includes the diffracted contributions from all Fresnel regions. Therefore, when FZPs are used in pulsed ultrasound systems, the pulse duration should be long enough to ensure that the steady state is reached, or degradation on the lens resolution should be expected, as the focus will never receive simultaneously the contributions of all the rings of the lens.

In this sense, if the transient state is defined from the propagation delay point of view and the first region is a pressure blocking ring, the transient state duration, $\Delta t$, can be calculated as the difference between the time of arrival at the focus of wavefront generated at the first radius and the wavefront generated at the last radius of the lens, that is,

$$
\Delta t=\frac{\sqrt{r_{N}^{2}+F^{2}}-\sqrt{r_{1}^{2}+F^{2}}}{c}=\frac{(N-1) \lambda}{2 c} .
$$

Figure 5.1 shows the transient state duration as a function of the wavelength for three different lens sizes: $N=11, N=21$, and $N=31$. As can be observed from the figure, the transient duration increases linearly with the wavelength, and thus higher frequency lenses will exhibit shorter transient times. Moreover, as the size of the lens increases, the transient duration increases too. It is worth noting that, as shown in Equation 5.2, the transient duration does not depend on the focal distance. This is a consequence of the design condition of the lens, that is, the path difference between consecutive regions is always $\lambda / 2$, independently on the focal distance of the lens.

\subsubsection{FZP Transient Response}

In order to analyze the transient state influence on the focusing parameters of the lens, i.e. axial and lateral resolutions, focal distance and focal intensity, a Soret lens with $N=21$ Fresnel regions, $\lambda=6 \mathrm{~mm}$, and a focal distance of $F=50$ 


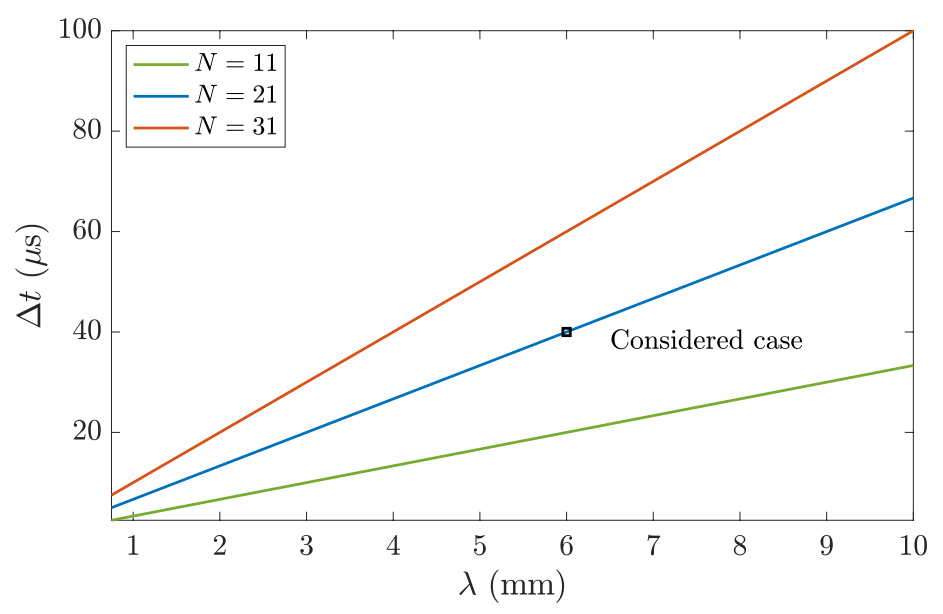

Figure 5.1: Transient state duration $\Delta t$ as a function of the wavelength: $N=11$ (green), $N=21$ (blue), and $N=31$ (red).

$\mathrm{mm}$ has been selected. A sound speed propagation in water of $c=1500 \mathrm{~m} / \mathrm{s}$ is considered, so $\lambda=6 \mathrm{~mm}$ is achieved for a frequency of $f=250 \mathrm{kHz}$. As shown in Figure 5.1, this lens provides a transient duration of $\Delta t=40 \mu \mathrm{s}$. Three different waveform shapes have been analyzed: an ideal Continuous Wave (CW) sinusoidal signal, a Modulated Rectangular Pulse (MRP), and a Modulated Gaussian Pulse (MGP). These waveform types can be described as

$$
\mathrm{CW}: x(t)=x_{0} \sin \left(2 \pi f_{0} t\right)
$$

$$
\begin{gathered}
\text { MRP: } x(t)=x_{0} \sin \left(2 \pi f_{0} t\right) \cdot \operatorname{rect}\left(\frac{t-T_{0} / 2}{T_{0}}\right) \\
\text { MGP: } x(t)=x_{0} \sin \left(2 \pi f_{0} t\right) \cdot e^{-\frac{\left(t-t_{0}\right)^{2}}{2 \sigma_{t}^{2}}}
\end{gathered}
$$

where $x_{0}$ is the signal amplitude, $f_{0}$ is the central frequency of the waveform, $\operatorname{rect}(\cdot)$ is the rectangular function, $T_{0}$ is the duration of the rectangular pulse, $\sigma_{t}$ is the standard deviation of the gaussian pulse, and $t_{0}$ is its offset starting time. The spectra of the three waveforms is given by the Fourier transform of the time-domain signal, which results in 


$$
\begin{gathered}
\mathrm{CW}: X(f)=\frac{x_{0}}{2 j}\left[\delta\left(f-f_{0}\right)-\delta\left(f+f_{0}\right)\right] \\
\begin{array}{r}
\text { MRP: } X(f)=\frac{x_{0} T_{0}}{2 j}\left[\operatorname{sinc}\left(T_{0}\left(f-f_{0}\right)\right) e^{-j \pi T_{0}\left(f-f_{0}\right)}\right. \\
\left.-\operatorname{sinc}\left(T_{0}\left(f+f_{0}\right)\right) e^{-j \pi T_{0}\left(f+f_{0}\right)}\right] \\
\operatorname{MGP:~} X(f)=\frac{x_{0} \sigma_{t} \sqrt{2 \pi}}{2 j}\left[e^{-2 \pi^{2} \sigma_{t}^{2}\left(f-f_{0}\right)^{2}} e^{-j 2 \pi t_{0}\left(f-f_{0}\right)}\right. \\
\left.-e^{-2 \pi^{2} \sigma_{t}^{2}\left(f+f_{0}\right)^{2}} e^{-j 2 \pi t_{0}\left(f+f_{0}\right)}\right]
\end{array}
\end{gathered}
$$

Figure 5.2 depicts the three considered waveforms in both time (left) and frequency (right) domains. The MRP duration is set to $T_{0}=60 \mu \mathrm{s}$, which ensures that the steady state will be achieved as $T_{0}>\Delta t$. On the other hand, the standard deviation of the MGP is set to $\sigma_{t}=30 \mu \mathrm{s}$. For this waveform case, the gaussian pulse duration is considered to be, approximately, $T_{0} \approx 2 \sigma_{t}$, which means that the MGP duration should also be enough to achieve the steady state.

The transient state of the lens is analyzed using the transversal and the longitudinal focusing profiles as a function of time. In cylindrical coordinates, the transient pressure distribution generated by the lens can be described by the pressure map $|P(r, z, t)|$, where $r$ represents the radial axis parallel to the lens and $z$ represents the longitudinal axis perpendicular to the lens. In this sense, the longitudinal focusing profile is defined as the pressure distribution along the central axis of the lens, that is, $|P(z, t)|=|P(r=0, z, t)|$, while the transversal focusing profile represents the pressure distribution at the focal distance, $|P(r, t)|=|P(r, z=F, t)|$. The transient pressure distribution, $|P(r, z, t)|$, has been calculated both theoretically and numerically. The theoretical approach is based on calculating the Rayleigh-Sommerfeld diffraction spectrum of the lens. In this method, the longitudinal and transversal focusing profiles are obtained in the frequency domain, $|P(z, f)|$ and $|P(r, f)|$, using as input information the lens radii and the excitation waveform spectra depicted in Figure 5.2, and then the transient profiles $|P(z, t)|$ and $|P(r, t)|$ are calculated as their inverse Fourier transform. The numerical results are obtained by solving a Finite Element Method (FEM) model with a transient solver (see more details at the Methods section).

Figure 5.3 depicts the longitudinal focusing profile spectra $|P(z, f)|$ for the three different waveforms of Figure 5.2 and their corresponding time responses 
(a)

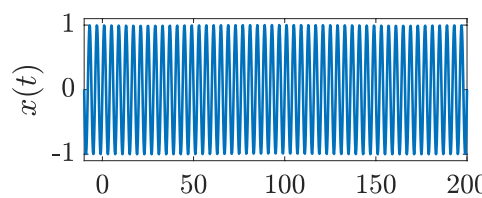

(b)

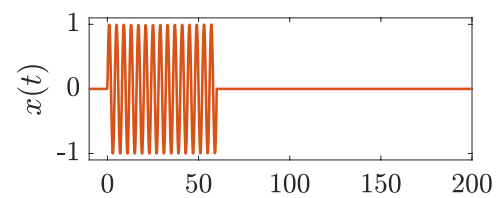

(c)

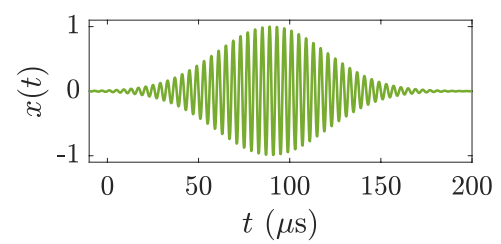

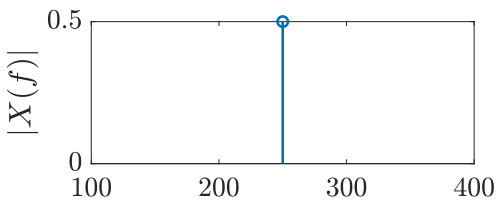
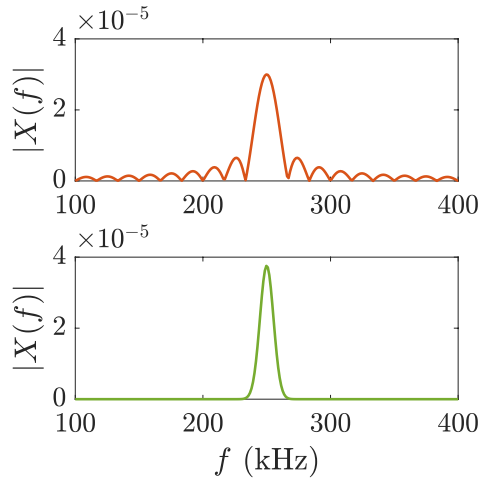

Figure 5.2: Waveforms (left) and their corresponding spectra (right): (a) Continuous Wave, (b) Modulated Rectangular Pulse of $T_{0}=60 \mu \mathrm{s}$, and (c) Modulated Gaussian Pulse of $\sigma_{t}=30 \mu \mathrm{s}$.

$|P(z, t)|$. As shown in Figure 5.3(a), the longitudinal spectrum of the lens with $\mathrm{CW}$ excitation has only values for the frequency of the continuous wave $f_{0}=250$ $\mathrm{kHz}$, as expected, because the frequency response of the $\mathrm{CW}$ is an ideal delta function centered at $f_{0}$. The time response $|P(z, t)|$ shows no transient response, as it represents the steady state of the lens. Figure 5.3(b) depicts the longitudinal spectrum for the MRP and its temporal response. In contrast to the CW case, the longitudinal profile of the MRP exhibits a clear transient response, then reaches a steady state, and finally another transient response until the MRP propagates away from the focus. The transient states of the MRP $|P(z, t)|$ profile show that the pressure is first focused in the focal area, and then spread out over the $z$-axis. Finally, Figure 5.3(c) depicts the longitudinal spectrum of the MGP case and its time response. In this MGP case, it is worth noting that during the transient state, the pressure is more focused on the focus than in the MRP case. This means that, for the MGP case the pressure is less dispersed along the axial distance and therefore, more spatially concentrated around the focus than for the MRP case during the transient states delimited between the first and the second, and the third and the fourth white lines of Figures 5.3(b) and 5.3(c).

In Figure 5.3, four white lines have been superimposed over the temporal $|P(z, t)|$ maps of the MRP and MGP cases, in order to highlight the three stage 
(a)
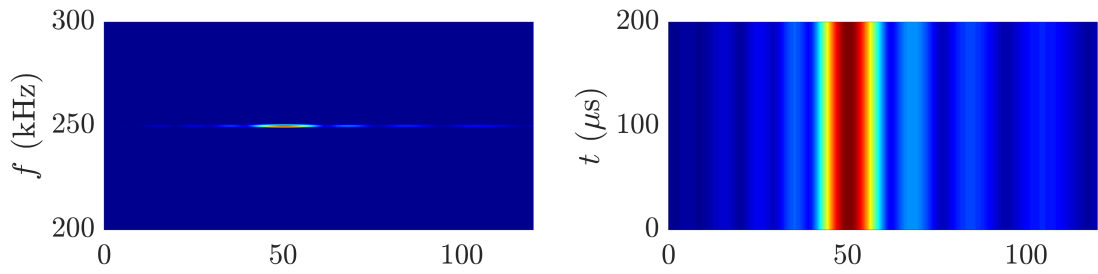

(b)
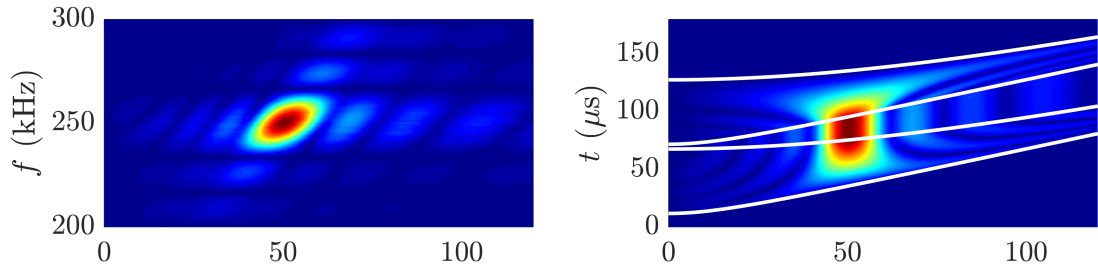

(c)
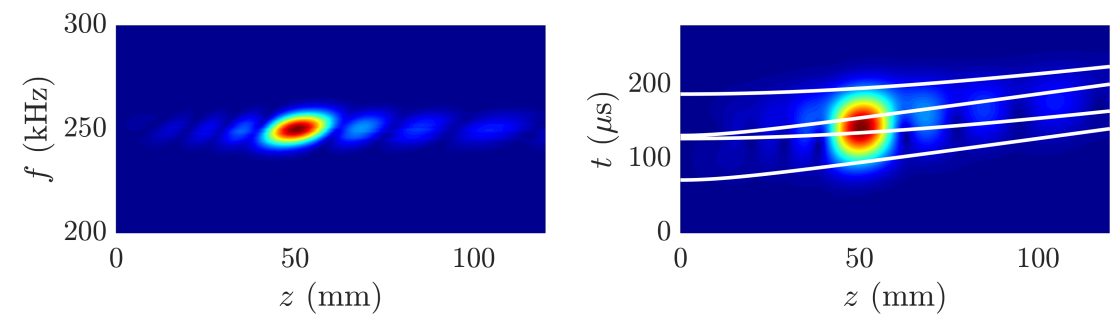

Figure 5.3: Longitudinal focusing profile spectra $|P(z, f)|$ (left) and transient focusing profile $|P(z, t)|$ (right): (a) Continuous Wave, (b) Modulated Rectangular Pulse, and (c) Modulated Gaussian Pulse.

response (transient-steady-transient). From bottom to top, the first line represents the incident wavefront diffracted from the first Fresnel region as a function of time, which sets the starting point of the transient response; and the second line represents the wavefront diffracted from the last Fresnel radius of the lens, and thus sets the ending point of the transient state and the starting point of the steady state. The position of the first line is therefore given by $t_{1}=\sqrt{r_{1}^{2}+z^{2}} / c$, while the second line is given by $t_{2}=\sqrt{r_{N}^{2}+z^{2}} / c$. Thus, for $z=F$ the difference between the second and the first line corresponds to the transient state duration as described by Equation 5.2. From that moment on, the wavefronts from all the lens regions are overlapped and contribute constructively to the focus. The third line represents the last wavefront from the first Fresnel region, due to the limited duration of the pulse, and its position is calculated as the first white line plus the duration of the pulse, that is, $t_{3}=t_{1}+T_{0}$. This third line sets the ending point of the steady state and the new starting point of the transient state. Thus, the steady state duration can be calculated as the difference between the third and the 
second line, which for the focal distance results in $t_{3}-t_{2}=T_{0}-\Delta t$. Finally, the fourth line represents the last wavefront from the last radius of the lens, located at $t_{4}=t_{2}+T_{0}$, and sets the end of the second transient stage.

Figure 5.4 shows the transversal focusing spectra $|P(r, f)|$ and time responses $|P(r, t)|$ for the same three cases of Figure 5.2. Similarly to Figure 5.3(a), Figure 5.4(a) shows that for the $\mathrm{CW}$ case the transversal spectrum has only content at the central frequency $f_{0}=250 \mathrm{kHz}$, and therefore the time response exhibits no transient states. On the other hand, Figures 5.4(b) and 5.4(c) show clear transient responses for the MRP and MGP cases, respectively. In these $|P(r, t)|$ pressure maps, the four white lines have the same meaning that in the $|P(z, t)|$ maps. In both Figures 5.4(b) and 5.4(c), the three stage response can be observed, with an initial transient state, followed by a steady response, and then another transient state.

(a)

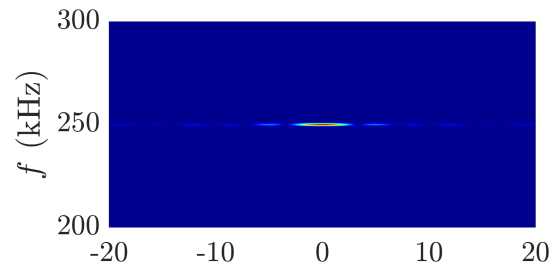

(b)

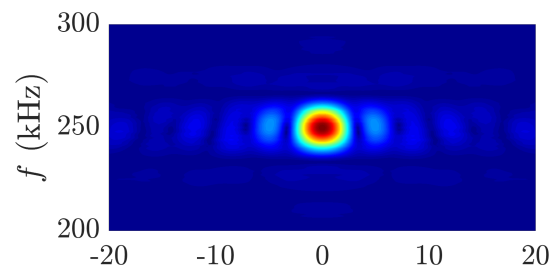

(c)

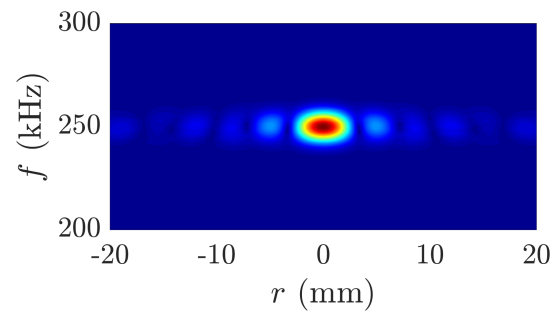

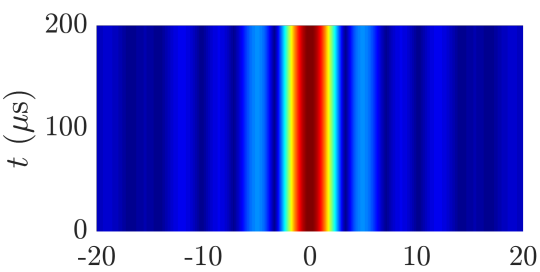
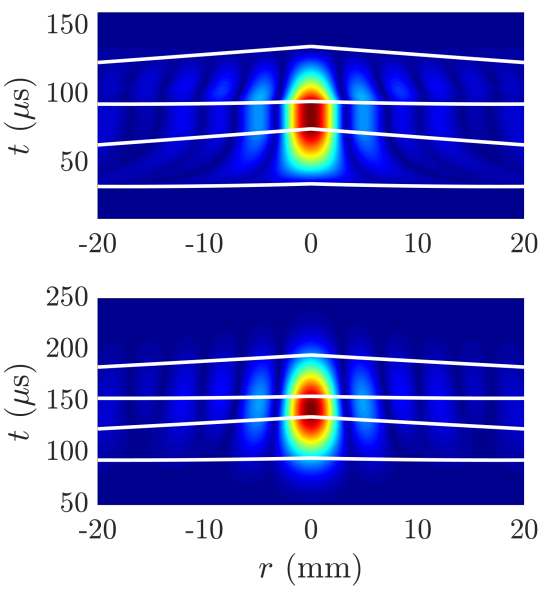

Figure 5.4: Transversal focusing profile spectra $|P(r, f)|$ (left) and transient focusing profile $|P(r, t)|$ (right): (a) Continuous Wave, (b) Modulated Rectangular Pulse, and (c) Modulated Gaussian Pulse.

Finally, the main focusing parameters have been analyzed. These parameters include: the pressure at the focal distance, the focal distance, the Full Length 
(a)
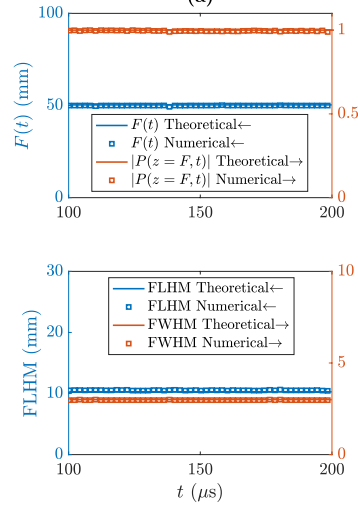

(b)
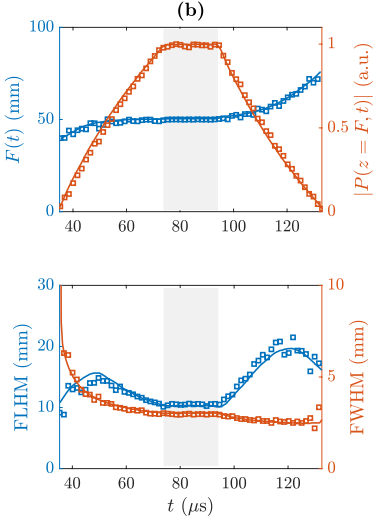

(c)
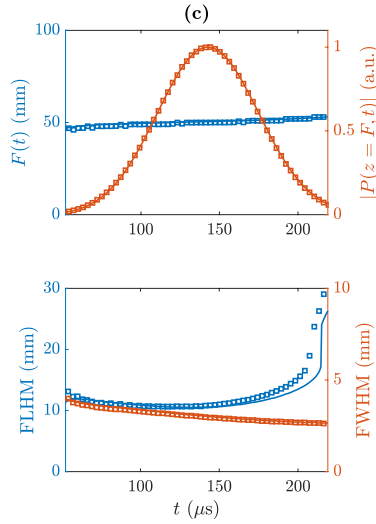

Figure 5.5: Main focusing parameters for the three different waveforms: (a) Continuous Wave, (b) Modulated Rectangular Pulse, and (c) Modulated Gaussian Pulse. Top plots depict the focal distance and focal intensity as a function of time, while bottom plots show FLHM and FWHM resolutions. Gray areas in (b) represent the transient state.

at Half Maximum (FLHM), and the Full Width at Half Maximum (FWHM). The FLHM specifies the lens resolution along the $z$-axis, while the FWHM specifies the resolution along the $r$-axis. Thus, Figure 5.5 depicts these four parameters as a function of time for the three considered waveforms. Solid lines represent theoretical results, whereas squares represent numerical results obtained using the FEM model. As can be observed from the figure, numerical and theoretical results are in very good agreement. The steady state parameters, obtained using the $\mathrm{CW}$ results depicted in Figure 5.5(a), result in a focal distance of $F=50 \mathrm{~mm}$, an axial resolution of $F L H M=10.3 \mathrm{~mm}$, and a lateral resolution of $\mathrm{FWHM}=3.0$ $\mathrm{mm}$. In the MRP case, depicted in Figure 5.5(b), the focal distance increases with the focal pressure, then reaches the steady state at its theoretical focal distance $F=50 \mathrm{~mm}$ with a sustained focal pressure, and finally increases again in the second transient state as the focal pressure decreases. The resolutions also show a first transient stage, then reach their steady resolutions (same as in the CW case), and finally another transient state. It is worth noting that, in the case of the lateral resolution (FWHM), the resolution increases at the first transient state (lower FWHM values), and also increases at the second transient state beyond its steady value of FWHM=3 $\mathrm{mm}$. This happens because the first transient is caused by the wavefronts generated at the inner Fresnel regions, which contain the lowest spatial frequencies (widest regions) and therefore, low lateral resolution information, while the second transient is generated by the outer Fresnel regions, which carry the highest spatial frequencies (narrowest regions) and therefore, high 
lateral resolution information. The steady state duration, which can be directly calculated either from the FLHM plot or the focal pressure plot in the MRP case, is approximately $20 \mu \mathrm{s}$, which agrees with the theoretical calculation of the pulse duration minus the transient state duration, $T_{0}-\Delta t=60-40=20 \mu \mathrm{s}$. Finally, the MGP waveform FZP response is shown at Figure 5.5(c). In this case, the transient state is not clearly visible. This phenomenon is a consequence of the gaussian shape of the waveform, which means that the pulses diffracted at the different Fresnel regions will not overlap exactly with the same amplitude, as each pulse has a different time of arrival to the focus, and therefore no steady focal pressure level can be achieved. This fact is also the reason why the steady FLHM is never achieved (CW case), and the MGP provides a maximum axial resolution of $\mathrm{FLHM}=10.5 \mathrm{~mm}$. However, as the MGP duration is long enough to cover the transient response state $\left(2 \sigma_{t}=60 \mu \mathrm{s}>\Delta t\right)$, the axial resolution is only reduced by $0.2 \mathrm{~mm}(\approx 0.03 \lambda)$, which is an acceptable degradation. As can be observed from the figure, the focal pressure has a gaussian shape and the focal distance increases linearly as a function of time, reaching the theoretical focal distance of $F=50 \mathrm{~mm}$ around the maximum focal pressure levels. The steady lateral resolution $\mathrm{FWHM}=3 \mathrm{~mm}$ is achieved around the maximum pressure value too and, as in the MRP case, the lateral resolution increases with time due to outer Fresnel regions carrying higher spatial frequencies than inner regions. Therefore, in both MRP and MGP waveform cases, if the duration of the pulse had been shorter than the transient duration $\Delta t$, the main focusing parameters of the lens would have been distorted: shifted focal distance, reduced focal pressure, and degraded axial and lateral resolutions.

\subsection{Methods}

\subsubsection{Rayleigh-Sommerfeld Spectrum}

The theoretical analysis of the FZP transient response is based on a two step process. First, the FZP response is calculated in the frequency domain using the Rayleigh-Sommerfeld integral, and then the transient response is calculated as an inverse Fourier transform. The Rayleigh-Sommerfeld diffraction integral is given by

$$
P(r, z, f)=\frac{X(f)}{j \lambda} \int_{0}^{2 \pi} \int_{0}^{r_{N}} t(\rho) \frac{e^{-j k R}}{R} \rho \cos \theta d \rho d \varphi
$$

where $X(f)$ is the waveform spectrum, $k=2 \pi f / c$ is the wavenumber, $(\rho, \varphi)$ 
are the radial and angular coordinates over the lens surface, $R$ is the distance between the lens and the $(r, z)$ point $\left(R=\sqrt{z^{2}+r^{2}+\rho^{2}-2 r \rho \cos \varphi}\right)$, and $\cos \theta=z / R$. The function $t(\rho)$ represents the transmission function of the lens, which for a Soret FZP type is 1 at the transparent Fresnel regions and 0 at the pressure blocking regions. Once the focusing spectrum is calculated, the transient response can be finally calculated as an inverse Fourier transform:

$$
P(r, z, t)=\int_{-\infty}^{+\infty} P(r, z, f) e^{j 2 \pi f t} d f .
$$

Equations 5.9 and 5.10 are both computed in MATLAB.

\subsubsection{Numerical Model}

The numerical model is based on a Finite Element Method (FEM) simulation using COMSOL Multiphysics 5.5. The model is solved in the time domain using a transient solver. The FZP lens is modelled as an axisymmetric model, with pressure conditions in the transparent Fresnel regions and sound hard boundary conditions at the pressure blocking regions. The pressure condition is set to the corresponding time-dependent waveform function under study. The water domain, modelled with density $\rho_{0}=1000 \mathrm{~kg} / \mathrm{m}^{3}$ and sound speed $c_{0}=1500 \mathrm{~m} / \mathrm{s}$, is surrounded by Perfectly Matched Layers (PMLs) in order to avoid reflections. The time-step of the solver is set to $1 /\left(60 f_{\max }\right)$, being $f_{\max }=f_{0}=250 \mathrm{kHz}$ the maximum frequency present in the model, to ensure convergence of the solution. The maximum mesh size is fixed to $\lambda_{0} / 6=1 \mathrm{~mm}$. After the solution is computed, the results are exported as a text file and then processed in MATLAB to obtain the main focusing parameters variation as a function of time.

\subsection{Conclusion}

Pulsed ultrasonic systems are widely used in NDT applications. In this kind of scenarios, the pulse duration is an important parameter that is used to control the axial resolution. This resolution can be further increased with passive ultrasonic lenses, such as FZPs. In this work, a transient analysis of FZP lenses is presented using both theoretical and numerical results, demonstrating that if the pulse duration is shorter than the transient state duration, degradation in both lateral and axial resolutions is observed. It is worth noting that, although this work is focused on FZPs, the same study could be carried out for other kind of ultrasound lenses, 
and a similar three stage transient-steady-transient response should be expected. Particularly, when planar lenses or 3D printed lenses attached to flat transducers are used, the propagation delay from the lens to the focal distance depends on the position along the lens surface, which will produce a transient response.

\section{References}

[1] S. Albu, "Potential for the use of ultrasound in the extraction of antioxidants from Rosmarinus officinalis for the food and pharmaceutical industry," Ultrasonics Sonochemistry, vol. 11, pp. 261-265, may 2004.

[2] J.-T. Li, J.-F. Han, J.-H. Yang, and T.-S. Li, "An efficient synthesis of 3,4dihydropyrimidin-2-ones catalyzed by $\mathrm{NH} 2 \mathrm{SO} 3 \mathrm{H}$ under ultrasound irradiation," Ultrasonics Sonochemistry, vol. 10, pp. 119-122, may 2003.

[3] D. McCann and M. Forde, "Review of NDT methods in the assessment of concrete and masonry structures," NDT \& E International, vol. 34, pp. 7184, mar 2001.

[4] J. Chen, J. Xiao, D. Lisevych, A. Shakouri, and Z. Fan, “Deep-subwavelength control of acoustic waves in an ultra-compact metasurface lens," Nature Communications, vol. 9, p. 4920, dec 2018.

[5] J.-L. Thomas and M. Fink, "Ultrasonic beam focusing through tissue inhomogeneities with a time reversal mirror: application to transskull therapy," IEEE Transactions on Ultrasonics, Ferroelectrics and Frequency Control, vol. 43, pp. 1122-1129, nov 1996.

[6] K. Melde, A. G. Mark, T. Qiu, and P. Fischer, "Holograms for acoustics," Nature, vol. 537, pp. 518-522, sep 2016.

[7] S. Castiñeira-lbáñez, D. Tarrazó-Serrano, J. Fuster, P. Candelas, and C. Rubio, "Polyadic Cantor Fractal Ultrasonic Lenses: Design and Characterization," Applied Sciences, vol. 8, p. 1389, aug 2018.

[8] C. Rubio, J. Fuster, S. Castiñeira-lbáñez, A. Uris, F. Belmar, and P. Candelas, "Pinhole Zone Plate Lens for Ultrasound Focusing," Sensors, vol. 17, p. 1690, jul 2017.

[9] Q. Zhou, Z. Xu, and X. Liu, "High efficiency acoustic Fresnel lens," Journal of Physics D: Applied Physics, vol. 53, p. 065302, feb 2020.

[10] D. Schindel, A. Bashford, and D. Hutchins, "Focussing of ultrasonic waves in air using a micromachined Fresnel zone-plate," Ultrasonics, vol. 35, pp. 275285, jun 1997. 
[11] D. C. Calvo, A. L. Thangawng, M. Nicholas, and C. N. Layman, "Thin Fresnel zone plate lenses for focusing underwater sound," Applied Physics Letters, vol. 107, p. 014103, jul 2015.

[12] D. Tarrazó-Serrano, S. Pérez-López, P. Candelas, A. Uris, and C. Rubio, "Acoustic Focusing Enhancement In Fresnel Zone Plate Lenses," Scientific Reports, vol. 9, p. 7067, dec 2019.

[13] J. Salazar, A. Turó, J. A. Chávez, J. A. Ortega, and M. J. García, "Transducer resolution enhancement by combining different excitation pulses," Ultrasonics, vol. 38, pp. 145-150, mar 2000.

[14] J. Salazar, A. Turo, J. Chavez, J. Ortega, and M. Garcia, "High-power highresolution pulser for air-coupled ultrasonic nde applications," IEEE Transactions on Instrumentation and Measurement, vol. 52, pp. 1792-1798, dec 2003.

[15] M. Oelze, "Bandwidth and resolution enhancement through pulse compression," IEEE Transactions on Ultrasonics, Ferroelectrics and Frequency Control, vol. 54, pp. 768-781, apr 2007.

[16] S. I. Konovalov and A. G. Kuz'menko, "On the optimization of the shapes of short-duration acoustic pulses for solving probing problems in immersion tests," Russian Journal of Nondestructive Testing, vol. 51, pp. 101-107, feb 2015 . 


\title{
Chapter 6
}

\section{Spatio-temporal Ultrasound Beam Modulation to Sequentially achieve Multiple Foci with a Single Planar Monofocal Lens}

\author{
S. Pérez-López, J. M. Fuster, and P. Candelas, "Spatio-temporal \\ ultrasound beam modulation to sequentially achieve multiple foci \\ with a single planar monofocal lens," Scientific Reports, vol. 11, \\ no. 1, p. 13458, Jun. 2021. \\ https://doi.org/10.1038/s41598-021-92849-x
}

\section{Abstract}

Ultrasound focusing is a hot topic due to its multiple applications in many fields, including biomedical imaging, thermal ablation of cancerous tissues, and non destructive testing in industrial environments. In such applications, the ability to control the focal distance of the ultrasound device in real-time is a key advantage over conventional devices with fixed focal parameters. Here, we present a method to achieve multiple time-modulated ultrasound foci using a single planar 
monofocal Fresnel Zone Plate. The method takes advantage of the focal distance linear dependence on the operating frequency of this kind of lenses to design a sequence of contiguous modulated rectangular pulses that achieve different focal distances and intensities as a function of time. Both numerical simulations and experimental results are presented, demonstrating the feasibility and potential of this technique.

\subsection{Introduction}

Focusing ultrasonic waves has multiple applications in various fields, such as nondestructive testing in industrial scenarios [1], biomedical imaging of different kind of tissues [2], or thermal ablation of tumours through High Intensity Focused Ultrasound (HIFU) [3-5]. Different techniques have been devised in the literature to achieve multiple acoustic foci. Among all the possible options, one of the main and most used methods is employing phased-arrays, which allows to generate different acoustic foci by adjusting the time delay of each one of its different transducer elements [6-8]. A more recent approach to achieve arbitrary pressure fields consists of using acoustic holograms directly coupled to a single ultrasound transducer, which provides a simpler and cheaper, yet very powerful and versatile method to provide complex 3D pressure distributions [9-14]. However, in contrast to phased-arrays, acoustic holograms do not allow to dynamically control the ultrasound beam once the lens is manufactured.

In this work, we present a spatio-temporal beam modulation technique to achieve multiple foci using a conventional Fresnel Zone Plate (FZP). With this method, an arbitrary number of foci and relative acoustic intensities can be multiplexed in the time domain, achieving, therefore, an ultrasonic focal beam that can be controlled in both space and time.

FZPs are widely used monofocal planar lenses made of a series of concentric rings, known as Fresnel regions, with decreasing width. Due to their easy design, manufacturing process, and focusing capabilities, this kind of devices are employed in a wide range of fields ranging from optics [15-17] to X-ray [18, 19], microwaves [20, 21], and acoustics [22-26]. Moreover, in the past years novel designs based on the FZP structure have been presented, which allow the formation of acoustic vortex [27], and bifocal [28, 29] and fractal [30,31] intensity distributions, increasing the versatility and interest of this type of lenses.

For a FZP lens, the diffracted pressure waves at two consecutive Fresnel regions reach the focus with a complex phase difference of $\pi$ (in phase opposition), meaning that those pressure waves interfere destructively. This $\pi$ phase change 
results in a propagation path difference of $\lambda / 2$ between consecutive radii, which directly yields to the design equation of the lens:

$$
d+F+\frac{n \lambda}{2}=\sqrt{d^{2}+r_{n}^{2}}+\sqrt{F^{2}+r_{n}^{2}},
$$

where $d$ is the distance between transducer and lens, $F$ is the focal distance, $\lambda$ is the working wavelength, $r_{n}$ is the $n$-th radius of the lens, and $n=1,2, \cdots, N$, being $N$ the total number of Fresnel regions. Two kinds of FZPs can be distinguished depending on the physical implementation of the phase-opposition regions. Thus, Soret FZPs alternate pressure blocking with transparent regions [23, 22, 26], while Phase-Reversal FZPs replace blocking areas with phase inverting regions [24, 32].

\subsection{Results}

One intrinsic property of FZPs is that they are designed to operate at a single frequency, as the different radii of the lens depend on the central design wavelength. When the frequency is reduced or increased from the central design frequency, there is a phase error among the pressure contributions of the different regions that yields to a focal shift from its theoretical focal distance. This focal shift results in undesired chromatic aberration in optical imaging scenarios [15], but in acoustics it can actually be used as a dynamic focal control method [33]. In fact, recently, researchers have introduced focal tunability to this kind of devices by shifting the operating frequency to control the focal distance of an airborne acoustic vortex generated by an active-spiral FZP [34], or by designing a stretchable silicon FZP [35].

Operating the FZP at frequencies around the central design frequency of the lens yields to a linear shift in the focus position with low distortion in terms of resolution and focal shape. One important parameter that can be used to characterize the frequency response of the lens is its axial spectrum, which represents the absolute value of the pressure profile (i.e. the pressure distribution along the central axis of the lens) as a function of the frequency. In this sense, Figure 6.1(a) represents the measured axial spectrum of a Soret FZP made of brass immersed in a distilled water tank, designed for $d=350 \mathrm{~mm}, F=50 \mathrm{~mm}, f=250 \mathrm{kHz}$ (which results in $\lambda=6 \mathrm{~mm}$ considering a speed of sound $c_{0}=1500 \mathrm{~m} / \mathrm{s}$ in water), and $N=27$. The lens was manufactured in brass due to its high impedance mismatch with water $\left(Z_{\text {brass }}=40\right.$ MRayls compared to $Z_{\text {water }}=1.5$ MRayls $)$, which ensures a good pressure blocking performance. However, despite this high impedance mismatch, a brass plate immersed in water presents a resonant be- 

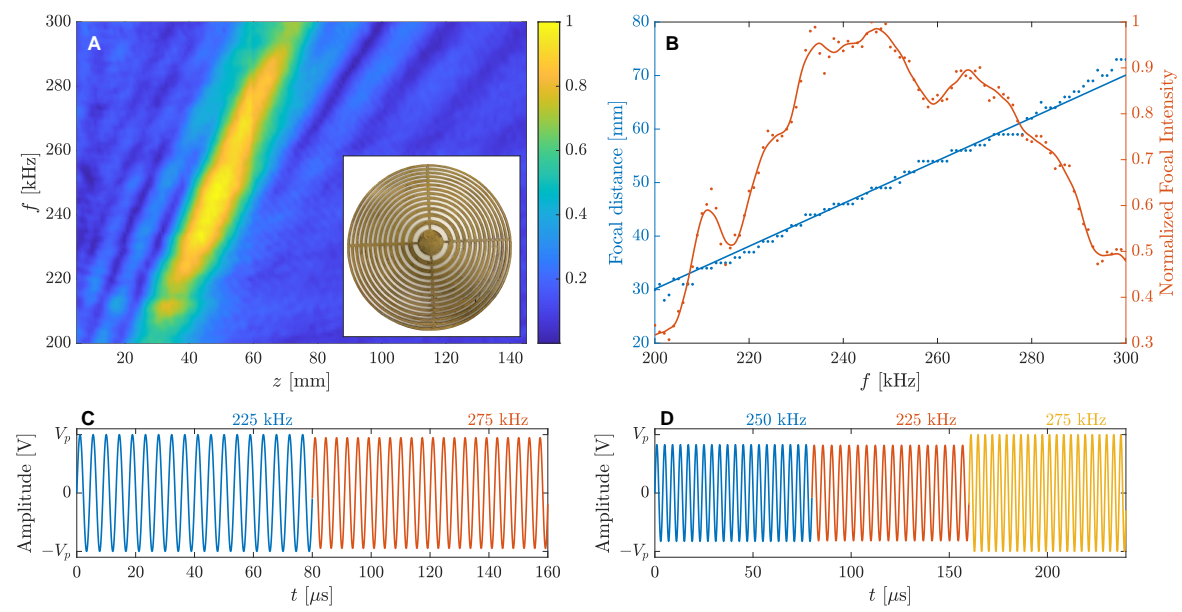

Figure 6.1: Time-modulation design technique: (A) measured axial spectrum of the Fresnel Zone Plate lens and (B) focal distance (left) and normalized focal intensity (right) as a function of the operating frequency. (C) Waveform designed to achieve two equal intensity foci at 40 and $60 \mathrm{~mm}$ and (D) waveform designed to achieve three foci at 50,40, and $60 \mathrm{~mm}$ with normalized intensities $0.8,0.6$, and 1 , respectively. In panel (B), solid dots correspond to experimental measurements while solid lines correspond to a linear fit to the data (blue) and a Savitzky-Golay interpolation filter (red).

haviour with full transmission coefficient at frequencies where the brass thickness is an integer multiple of $\lambda_{\text {brass }} / 2$, which means that the thickness of the lens has to be selected so that the resonant frequencies are far away from the operating frequency range. In this case, the manufactured lens has a maximum radius of $104.4 \mathrm{~mm}$ and a thickness of $t_{h}=1 \mathrm{~mm}$, which provides a first resonant mode at 2.35 MHz. The measurements were carried out using an automated 3D scanning system with a $1 \mathrm{~mm}$ needle hydrophone from Precision Acoustics Ltd. The signal was generated using an Arbitrary Waveform Generator (AWG) connected to a 75 W power amplifier and an Imasonic $250 \mathrm{kHz}$ piston transducer with an aperture of $30 \mathrm{~mm}$ (see Supplementary Information for more details on the axial spectrum measurement process). It is worth noting that the axial spectrum is influenced by the frequency response of the transducer, which affects the amplitude of the transmitted pressure depending on the frequency of the input waveform.

Due to the planar geometry of the FZP, the system will present a transient response [36], as the contributions of the different Fresnel regions will reach the focus at different times due to the distance difference in their propagation 
paths. Thus, the steady state is reached when the pressure waves diffracted at the different regions of the lens overlap at the focus simultaneously. In this case, the transient state duration $(\Delta t)$ can be calculated as the difference between the time of arrival of the pressure wave diffracted at the first Fresnel region (shortest propagation path) and the time of arrival of the pressure wave diffracted at the last Fresnel region (longest propagation path), that is,

$$
\Delta t=\frac{\left(\sqrt{d^{2}+r_{27}^{2}}+\sqrt{F^{2}+r_{27}^{2}}\right)-\left(\sqrt{d^{2}+r_{1}^{2}}+\sqrt{F^{2}+r_{1}^{2}}\right)}{c_{0}} .
$$

This transient state duration represents the maximum response speed of the system. It is worth noting that the transient state duration only depends on the geometric properties of the system, and not on the operating frequency. The transient state duration for the 40,50 , and $60 \mathrm{~mm}$ foci are 55.62, 52.00, and $48.72 \mu \mathrm{s}$, respectively.

As can be observed from Figure 6.1(a), the lens presents a single main pressure focus located at its theoretical design focal distance of $50 \mathrm{~mm}$ when the operating frequency corresponds to the design frequency of $250 \mathrm{kHz}$, which is shifted towards the lens when the frequency is reduced and away from the lens when the frequency is increased. Moreover, as can be seen in Figure 6.1(a), the maximum pressure value is achieved around the central frequency. Figure 6.1(b) depicts the focal distance and the normalized focal intensity as a function of the operating frequency. From Figure 6.1(b), the modulation bandwidth (BW) of the system can be calculated as the frequency span where the focal intensity is equal or higher than half of the maximum focal intensity value. Therefore, the system provides an experimental modulation BW of $85 \mathrm{kHz}$ (34\% respect to the central design frequency) ranging from 208 to $293 \mathrm{kHz}$, which results in an achievable focal distance range that goes from 33 to $68 \mathrm{~mm}$ (70\% respect to the central FZP design focal distance). By using these results, it is possible to create an input waveform of contiguous rectangular pulses that can modulate the spatial response of the lens in the time domain, achieving an arbitrary number of foci and relative acoustic intensities. For this, first, the focal distances, normalized intensities, and time sequence of the foci are selected. Then, the pulse width has to be configured such that its duration is longer than the transient state duration and the steady response of the lens can be achieved [36]. Next, Figure 6.1(b) is used to select the frequencies and amplitudes that provide the desired focal distances and normalized intensities. The pulse amplitude is calculated as $V_{i}=1 / \sqrt{I_{F}\left(f_{i}\right)}$, being $I_{F}\left(f_{i}\right)$ the normalized focal intensity and $f_{i}$ the frequency that provides the design focal distance $F_{i}$. Finally, the waveform is formed by concatenating rectangular pulses with the calculated frequencies and amplitudes in the desired 


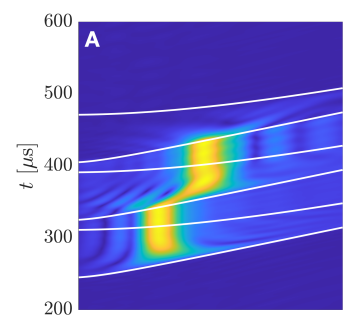

$\begin{array}{lllll}20 & 40 & 60 & 80 & 100\end{array}$

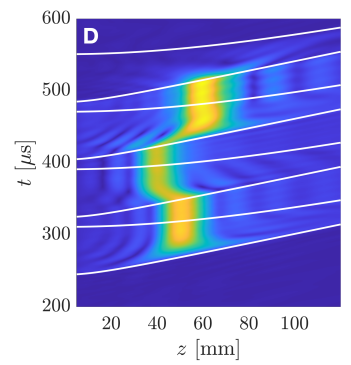

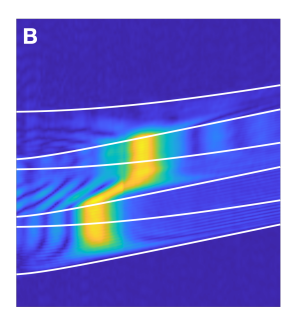
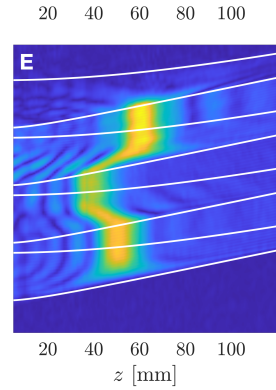
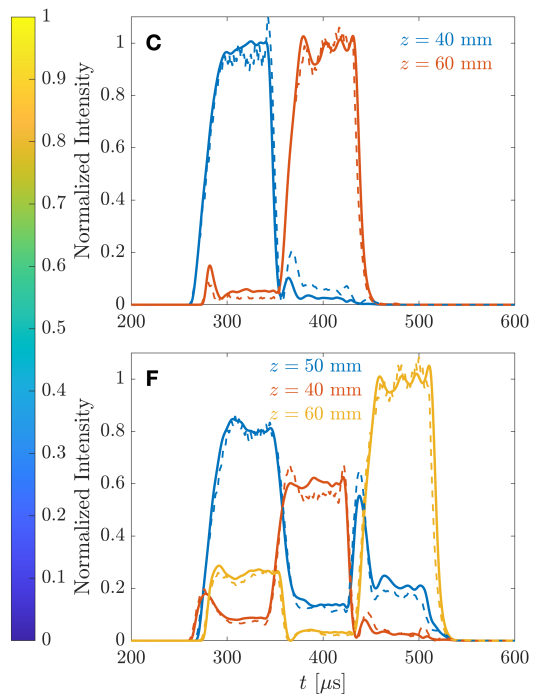

Figure 6.2: Simulated and measured results for the two waveforms depicted in panels 6.1(C) and 6.1(D): (A) simulated and (B) measured pressure profile as a function of time using the two foci waveform, and (C) focal intensities as a function of time. (D) simulated and (E) measured pressure profile as a function of time using the three foci waveform, and (F) focal intensities as a function of time. White lines in panels (A), (B), (D), and (E) represent the beginning and ending of transient states. In panels $(C)$ and $(F)$, solid lines represent simulation results, while dashed lines are for measurements.

time order, and normalized so its maximum amplitude corresponds to the desired peak voltage $\left(V_{p}\right)$. In this sense, Figure $6.1(\mathrm{c})$ represents an example of a waveform designed to achieve focal distances of $F=\{40,60\} \mathrm{mm}$ with normalized focal intensities of $I_{F}=\{1,1\}$, whereas Figure 6.1(d) depicts a waveform calculated for $F=\{50,40,60\} \mathrm{mm}$ and $I_{F}=\{0.8,0.6,1\}$. The pulse duration in Figures $6.1(\mathrm{c})$ and $6.1(\mathrm{~d})$ is set to $80 \mu \mathrm{s}$, which ensures reaching a steady lens response. These waveforms would allow focusing an ultrasound beam at two and three focal distances within a single scan, respectively. However, it is worth noting that one drawback of this method is that it does not allow to achieve all the foci at the same time using a single continuous wave with all the required frequency components added simultaneously, due to the phase interference pattern among the different complex pressure profiles of each frequency (see Supplementary Information for more details).

One important parameter of every acoustic focusing system is its spatial 
resolution in both axial (perpendicular to the lens) and lateral (parallel to the lens) directions. In this sense, the proposed spatio-temporal modulation technique does not allow to control the resolution of the system by shifting the operating frequency, in contrast to the focal distance and focal intensity. Therefore, once the FZP is manufactured, the resolution of the system is constrained, meaning that, for a given focal distance, the resolution is fixed and cannot be controlled independently from the focal distance. However, due to the diffractive nature of FZPs, increasing the size of the lens, and therefore the number of Fresnel regions, will provide a focus with higher resolution. Thus, the number of Fresnel regions has to be selected according to the spatial resolution specifications required for each particular application. In this case, the system achieves an experimental lateral resolution of approximately $3.5 \mathrm{~mm}$, for the full frequency range, while the axial resolution ranges from 12 to $18 \mathrm{~mm}$ (see Supplementary Information for more details).

Figure 6.2 depicts the experimental results measured using the waveforms represented in panels 6.1 (c) and $6.1(\mathrm{~d})$, compared to ideal numerical simulations computed using Equation 6.3, showing very good agreement between simulations and experiments. The measured pressure profiles have been calculated as the absolute value of the complex Hilbert transform of the measured signal, for each one of the scanning positions along the central axis of the lens. Figures 6.2(a) and 6.2(b) represent the simulated and measured profiles as a function of time for the two foci waveform case, respectively, while Figure 6.2(c) depicts the focal intensities as a function of time for each focus. Time spans in Figure 6.2 start at $200 \mu \mathrm{s}$ because the propagation delay from the transducer to the shortest focal distance $(F=40 \mathrm{~mm})$ is $262.41 \mu \mathrm{s}$. As can be observed in panels $6.2(\mathrm{a})$ and $6.2(\mathrm{~b})$, the pressure profile presents an initial transient state, depicted between the first two white lines (in time order), and then a steady response with a focus located at the first design focal distance $(F=40 \mathrm{~mm})$. As mentioned before, this transient response is a consequence of the planar shape of the lens, which results in diffracted pressure pulses with longer propagation paths for the outer Fresnel regions than for the inner regions, and therefore the lens only achieves its steady response once the pressure contributions from all regions overlap at the focus. Next, once the first rectangular pulse ends after $80 \mu$ s and the second pulse designed to focus at $F=60 \mathrm{~mm}$ starts, the pressure profile presents another transient state, depicted between the third and fourth white lines, where the pressure focus shifts from the first to the second focal distance. Then, the lens achieves a steady focus at its designed second focal distance $(F=60 \mathrm{~mm})$, followed by one last transient state depicted between the fifth and sixth white lines. Therefore, the transition state duration from one focus to the next is directly given by the corresponding transient state duration. As shown in panel 6.2(c), both foci reach approximately the same intensity level, which agrees with the design goal. Analogously, Figures 6.2(d), 6.2(e), and 6.2(f) show the results for the three foci waveform case. As expected, 

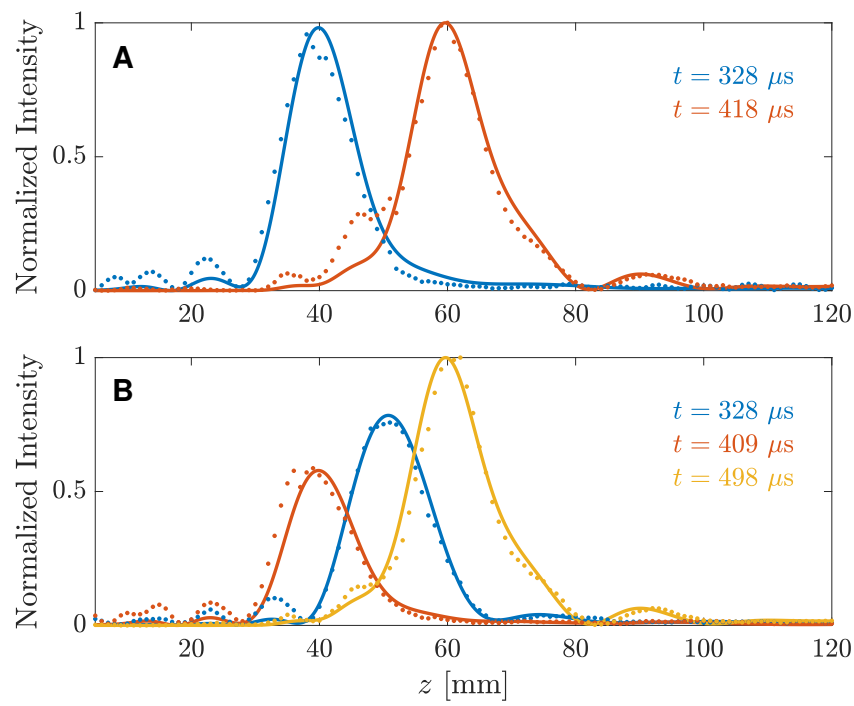

Figure 6.3: Steady focusing profiles: (A) two foci waveform and (B) three foci waveform. Solid lines represent simulation results, while dots represent experimental measurements.

the lens first focuses the ultrasound beam at $F=50 \mathrm{~mm}$ with a normalized intensity level of $I_{F} \approx 0.8$, then shifts to $F=40 \mathrm{~mm}$ with $I_{F} \approx 0.6$, and finally focuses at $F=60 \mathrm{~mm}$ with $I_{F} \approx 1$. However, some cross-talk among the different foci can be observed in Figure 6.2(f), due to the limited axial resolution. This means that, for instance, the $F=50 \mathrm{~mm}$ focus presents an axial spread wide enough to generate intensity signal at both $F=40$ and $F=60 \mathrm{~mm}$ foci.

Figure 6.3 depicts the steady intensity profiles compared to their ideal simulations, showing again good agreement between experiments and numerical calculations. As can be seen from the results, both waveforms depicted in Figures 6.1(c) and 6.1(d) achieve their corresponding design goal in terms of both focal distance and normalized intensity. The measured axial resolutions for the 40, 50, and $60 \mathrm{~mm}$ foci are 12.81, 14.11, and $13.02 \mathrm{~mm}$, respectively. 


\subsection{Discussion}

In summary, the proposed design method allows to modulate in space and time the response of a conventional monofocal FZP, achieving multiple ultrasound foci with arbitrary focal intensities. Experimental measurements and numerical simulations have been presented for two different case examples, one with two equal intensity foci and other with three foci with different acoustic intensities, demonstrating the potential of the proposed technique.

As mentioned earlier, due to the diffractive nature of the lens, once the lens is manufactured, the resolution is fixed and cannot be dynamically tuned for each focal distance. Therefore, if a higher axial or lateral resolution is required for a particular application, the number of Fresnel regions of the FZP should be increased. Alternatively, to increase the spatial resolution of the system while reducing its size, a higher frequency range could be employed, as the spatial properties of the system (size and resolution) are proportional to the central design wavelength. The drawbacks of increasing the frequency are mainly associated to the fact that RF electronics become more complicated and to the fact that higher accuracy and spatial resolution are required to manufacture the lens. In addition, ultrasound attenuation increases when using higher frequencies, which can be limiting in some biomedical scenarios. Another limitation of the proposed spatiotemporal modulation technique is the achievable focal distance range, which is limited by the modulation BW. In this sense, the modulation BW is limited by the combination of two factors: the BW of the transducer and the distortion introduced by the FZP itself, as a consequence of operating the lens at a frequency different from its original design frequency. Therefore, to increase the modulation BW, a transducer with a higher BW could be employed, or an FZP with a lower number of Fresnel regions (at the expense of reducing the spatial resolution of the system).

The method can be implemented in current ultrasound focusing systems where FZP lenses are employed, at a low cost, as only an AWG is required to generate the input waveforms. Although the proposed method is not able to focus simultaneously at all the focal distances, it is worth keeping in mind that the duration and amplitudes of each focus can be controlled individually, and even the delay between pulses could be tuned to provide specific target pressure profiles. This has potential applications in non-destructive testing or medical imaging where multiple focal planes could be scanned within one single pulse. In addition, the method could be used in medical ultrasound scenarios, creating ablation patterns with different tissue depths, allowing to specify individual ablation times and intensities for each target area. 


\subsection{Materials and Methods}

\subsubsection{Experimental Set-up}

The experimental set-up consists of a $1 \mathrm{~mm}$ needle hydrophone from Precision Acoustics Ltd. attached to a robotic arm that can move along the 3 spatial axis with a resolution of $1 \times 1 \times 1 \mathrm{~mm}^{3}$. The robotic arm is connected to an automated positioning system controlled by a PC with a National Instruments NI7330 controller card and a custom-made LabView software that automatically controls the measurement and scanning process. The signal is digitized using a PicoScope 3224 USB digital oscilloscope. The transmitted signal is generated using an Agilent 33220A Arbitrary Waveform Generator (AWG) controlled by a PC with MATLAB R2018a (MathWorks Inc.). The output of the AWG is connected to a $75 \mathrm{~W}$ power amplifier 75A250A from Amplifier Research, and then to a 30 $\mathrm{mm}$ aperture immersion transducer from Imasonic, with a central frequency of $250 \mathrm{kHz}$. Figure 6.4 shows a picture of the experimental set-up. Before starting the measurements, a cross-shape self-leveling laser is used to spatially align the hydrophone with the lens and the transducer, as depicted in the right side of Figure 6.4 .

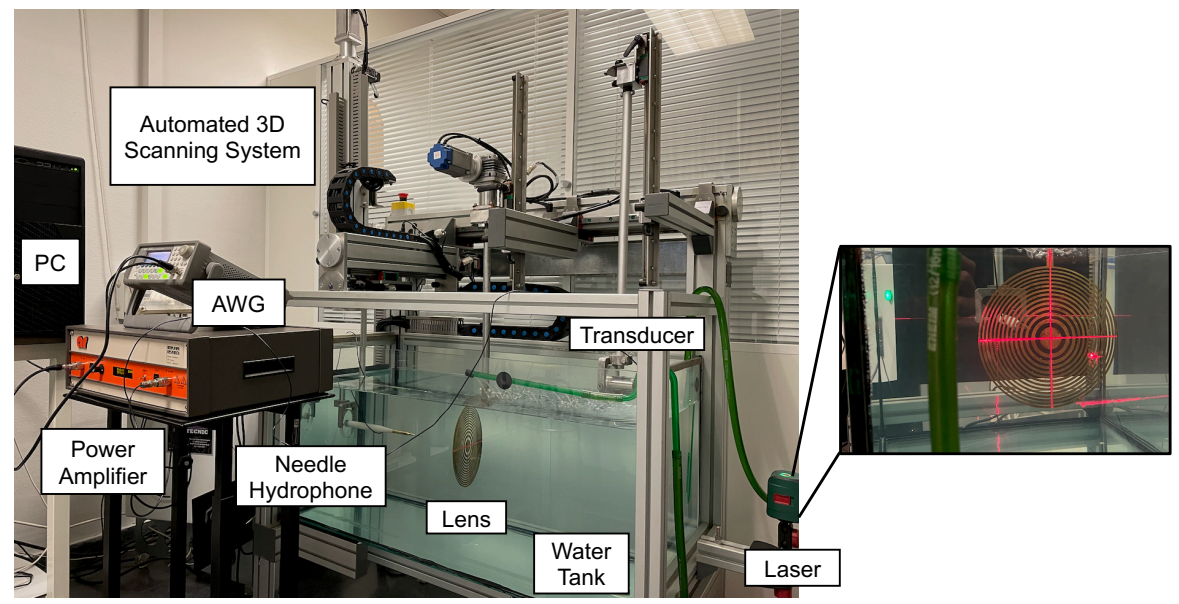

Figure 6.4: Picture of the experimental set-up (left side) and inset with a picture of the system position and level calibration using an self-leveling laser device (right side). 


\subsubsection{Axial Spectrum Simulation}

The axial spectrum of a FZP, given by $\left|p_{z}(\omega, z)\right|$, can be calculated using the Rayleigh-Sommerfeld diffraction integral [37] evaluated at the central axis of the lens, which eliminates the azimuthal dependence of the 2D integral over the lens surface due to its rotational symmetry and yields to the simplified expression

$$
p_{z}(\omega, z)=-j k X(\omega) z \int_{0}^{+\infty} p_{i}(\rho) t(\rho) \frac{e^{j k \sqrt{\rho^{2}+z^{2}}}}{\rho^{2}+z^{2}} \rho d \rho
$$

where $X(\omega)$ is the input waveform spectrum, $k=\omega / c_{0}$ is the wavenumber, $p_{i}(\rho)$ is the incident pressure at the lens, $t(\rho)$ is the transmittance or pupil function of the lens (which is 1 at the transparent regions and 0 at the pressure blocking regions), and $\rho$ is the radial axis along the surface of the lens. Once the axial spectrum is calculated for each input waveform, the transient response of the lens is calculated as the inverse Fourier transform of the spectrum, $p_{z}(t, z)=\left|\mathcal{F}_{\omega}^{-1}\left\{p_{z}(\omega, z)\right\}\right|$.

\section{References}

[1] L. W. Schmerr, Fundamentals of Ultrasonic Nondestructive Evaluation. Springer Series in Measurement Science and Technology, Cham: Springer International Publishing, 2016.

[2] H. Azhari, Basics of Biomedical Ultrasound for Engineers. Hoboken, NJ, USA: John Wiley \& Sons, Inc., feb 2010.

[3] X. Fan and K. Hynynen, "Ultrasound surgery using multiple sonications-Treatment time considerations," Ultrasound in Medicine \& Biology, vol. 22, pp. 471-482, jan 1996.

[4] G. ter Haar and C. Coussios, "High intensity focused ultrasound: Physical principles and devices," International Journal of Hyperthermia, vol. 23, pp. 89-104, jan 2007.

[5] Sijia Guo, Yun Jing, and Xiaoning Jiang, "Temperature rise in tissue ablation using multi-frequency ultrasound," IEEE Transactions on Ultrasonics, Ferroelectrics, and Frequency Control, vol. 60, pp. 1699-1707, aug 2013.

[6] E. Ebbini and C. Cain, "Multiple-focus ultrasound phased-array pattern synthesis: optimal driving-signal distributions for hyperthermia," IEEE Transactions on Ultrasonics, Ferroelectrics and Frequency Control, vol. 36, pp. 540548, sep 1989. 
[7] A. Casper, Dalong Liu, and E. S. Ebbini, "Realtime Control of Multiple-focus Phased Array Heating Patterns Based on Noninvasive Ultrasound Thermography," IEEE Transactions on Biomedical Engineering, vol. 59, pp. 95-105, jan 2012 .

[8] A. llovitsh, T. llovitsh, J. Foiret, D. N. Stephens, and K. W. Ferrara, "Simultaneous Axial Multifocal Imaging Using a Single Acoustical Transmission: A Practical Implementation," IEEE Transactions on Ultrasonics, Ferroelectrics, and Frequency Control, vol. 66, pp. 273-284, feb 2019.

[9] R. Lalonde, A. Worthington, and J. Hunt, "Field conjugate acoustic lenses for ultrasound hyperthermia," IEEE Transactions on Ultrasonics, Ferroelectrics and Frequency Control, vol. 40, pp. 592-602, sep 1993.

[10] R. Lalonde and J. Hunt, "Variable frequency field conjugate lenses for ultrasound hyperthermia," IEEE Transactions on Ultrasonics, Ferroelectrics and Frequency Control, vol. 42, pp. 825-831, sep 1995.

[11] M. D. Brown, T. J. Allen, B. T. Cox, and B. E. Treeby, "Control of optically generated ultrasound fields using binary amplitude holograms," in IEEE International Ultrasonics Symposium, IUS, pp. 1037-1040, IEEE, sep 2014.

[12] K. Melde, A. G. Mark, T. Qiu, and P. Fischer, "Holograms for acoustics," Nature, vol. 537, pp. 518-522, sep 2016.

[13] M. D. Brown, B. T. Cox, and B. E. Treeby, "Design of multi-frequency acoustic kinoforms," Applied Physics Letters, vol. 111, p. 244101, dec 2017.

[14] S. Jiménez-Gambín, N. Jiménez, J. M. Benlloch, and F. Camarena, "Holograms to Focus Arbitrary Ultrasonic Fields through the Skull," Physical Review Applied, vol. 12, p. 014016, jul 2019.

[15] M. Young, "Zone Plates and Their Aberrations," Journal of the Optical Society of America, vol. 62, p. 972, aug 1972.

[16] R. S. Rodrigues Ribeiro, P. Dahal, A. Guerreiro, P. A. S. Jorge, and J. Viegas, "Fabrication of Fresnel plates on optical fibres by FIB milling for optical trapping, manipulation and detection of single cells," Scientific Reports, vol. 7, p. 4485 , dec 2017 .

[17] H. Kim, J. Kim, H. An, Y. Lee, G.-y. Lee, J. Na, K. Park, S. Lee, S.-Y. Lee, B. Lee, and Y. Jeong, "Metallic Fresnel zone plate implemented on an optical fiber facet for super-variable focusing of light," Optics Express, vol. 25, p. 30290, nov 2017.

[18] J. Kirz, "Phase Zone Plates for X Rays and the Extreme Uv.," J Opt Soc Am, vol. 64, pp. 301-309, mar 1974. 
[19] W. Yashiro, Y. Takeda, A. Takeuchi, Y. Suzuki, and A. Momose, "Hard-XRay Phase-Difference Microscopy Using a Fresnel Zone Plate and a Transmission Grating," Physical Review Letters, vol. 103, p. 180801, oct 2009.

[20] H. D. Hristov and M. H. Herben, "Millimeter-Wave Fresnel-Zone Plate Lens and Antenna," IEEE Transactions on Microwave Theory and Techniques, vol. 43, no. 12, pp. 2779-2785, 1995.

[21] H. D. Hristov and J. M. Rodriguez, "Design Equation for Multidielectric Fresnel Zone Plate Lens," IEEE Microwave and Wireless Components Letters, vol. 22, pp. 574-576, nov 2012.

[22] G. Chao, B. A. Auld, and D. K. Winslow, "Focusing and Scanning of Acoustic Beams With Fresnel Zone Plates.," in 1972 Ultrasonics Symposium, pp. 140143, IEEE, 1972.

[23] S. A. Farnow and B. A. Auld, "Acoustic Fresnel zone plate transducers," Applied Physics Letters, vol. 25, pp. 681-682, dec 1974.

[24] S. A. Farnow and B. A. Auld, "An Acoustic Phase Plate Imaging Device," in Acoustical Holography, vol. 6, pp. 259-273, Boston, MA: Springer US, 1975.

[25] K. Yamada and H. Shimizu, "Planar-Structure Focusing Lens for Acoustic Microscope.," in Ultrasonics Symposium Proceedings, pp. 755-758, IEEE, 1985.

[26] D. C. Calvo, A. L. Thangawng, M. Nicholas, and C. N. Layman, "Thin Fresnel zone plate lenses for focusing underwater sound," Applied Physics Letters, vol. 107, p. 014103, jul 2015.

[27] N. Jiménez, V. Romero-García, L. M. García-Raffi, F. Camarena, and K. Staliunas, "Sharp acoustic vortex focusing by Fresnel-spiral zone plates," Applied Physics Letters, vol. 112, p. 204101, may 2018.

[28] J. A. Monsoriu, A. Calatayud, L. Remon, W. D. Furlan, G. Saavedra, and P. Andres, "Bifocal Fibonacci Diffractive Lenses," IEEE Photonics Journal, vol. 5, pp. 3400106-3400106, jun 2013.

[29] S. Pérez-López, J. M. Fuster, and P. Candelas, “M-Bonacci Zone Plates for Ultrasound Focusing," Sensors, vol. 19, p. 4313, oct 2019.

[30] G. Saavedra, W. D. Furlan, and J. A. Monsoriu, "Fractal zone plates," Optics Letters, vol. 28, p. 971, jun 2003.

[31] S. Pérez-López, J. M. Fuster, P. Candelas, and C. Rubio, "Fractal lenses based on Cantor binary sequences for ultrasound focusing applications," Ultrasonics, vol. 99, p. 105967, nov 2019. 
[32] D. Tarrazó-Serrano, S. Pérez-López, P. Candelas, A. Uris, and C. Rubio, "Acoustic Focusing Enhancement In Fresnel Zone Plate Lenses," Scientific Reports, vol. 9, p. 7067, dec 2019.

[33] J. M. Fuster, P. Candelas, S. Castiñeira-lbáñez, S. Pérez-López, and C. Rubio, "Analysis of fresnel zone plates focusing dependence on operating frequency," Sensors (Switzerland), vol. 17, p. 2809, dec 2017.

[34] R. D. Muelas-Hurtado, J. L. Ealo, and K. Volke-Sepúlveda, "Active-spiral Fresnel zone plate with tunable focal length for airborne generation of focused acoustic vortices," Applied Physics Letters, vol. 116, p. 114101, mar 2020.

[35] X. Xia, Y. Li, F. Cai, H. Zhou, T. Ma, and H. Zheng, "Ultrasonic tunable focusing by a stretchable phase-reversal Fresnel zone plate," Applied Physics Letters, vol. 117, p. 021904, jul 2020.

[36] S. Pérez-López, D. Tarrazó-Serrano, D. O. Dolmatov, C. Rubio, and P. Candelas, "Transient Analysis of Fresnel Zone Plates for Ultrasound Focusing Applications," Sensors, vol. 20, p. 6824, nov 2020.

[37] Dong-Lai Liu and R. Waag, "Propagation and backpropagation for ultrasonic wavefront design," IEEE Transactions on Ultrasonics, Ferroelectrics and Frequency Control, vol. 44, pp. 1-13, jan 1997.

\section{Supplementary Information}

\section{Simultaneous multifocal simulations}

One of the main drawbacks of the proposed method is that, although it can provide multiple foci, it cannot provide them simultaneously. This is due to the intrinsic nature of the complex frequency response of the lens. One could think that, for instance, the resulting focusing profile of two simultaneous signals with different frequencies added together would result in the combination of the two corresponding intensity focusing profiles for each frequency. But this is not possible, as the combination of both focusing profiles is not calculated using the intensity profiles but the complex pressure profiles, and therefore the phase response of both profiles interfere with each other resulting in a time-varying intensity distribution. Thus, in the steady state each profile can be described as $p_{n}(z, t)=p_{n}(z) e^{j \omega_{n} t}$, being $p_{n}(z)$ the absolute axial pressure distribution and $e^{j \omega_{n} t}$ the harmonic time dependent term. Let's consider the case of a $p(z, t)$ complex pressure distribution 
made from the combination of two profiles with different frequencies. The norm of such pressure profile can be expressed as

$$
\begin{aligned}
|p(z, t)| & =\left|p_{1}(z, t)+p_{2}(z, t)\right|=\left|p_{1}(z) e^{j \omega_{1} t}+p_{2}(z) e^{j \omega_{2} t}\right| \\
& =\sqrt{p_{1}(z)^{2}+p_{2}(z)^{2}+2 p_{1}(z) p_{2}(z) \cos \left(\left(\omega_{2}-\omega_{1}\right) t\right)},
\end{aligned}
$$

which presents a time dependent term with frequency $\omega_{2}-\omega_{1}$ that will result in a time varying pressure profile, therefore highly distorting the target pressure distribution. To showcase this phenomenon, Figure 6.5 shows simulations of two waveforms using the same frequencies and amplitudes as those calculated in the main manuscript to achieve two equal intensity foci $\left(F=\{40,60\} \mathrm{mm}\right.$ and $I_{F}=$ $\{1,1\})$ and three foci with different amplitudes $\left(F=\{40,50,60\} \mathrm{mm}\right.$ and $I_{F}=$ $\{0.6,0.8,1\})$, but using a waveform that simultaneously presents all the frequency and amplitude components. For this simulation, continuous wave excitation is considered, so only steady state responses are shown. As can be observed from the results, the focusing profiles are highly distorted and time dependent, and they do not provide the desired two and three foci configuration, due to the interference among the different frequency components. In contrast, the proposed method in the main manuscript, which employs contiguous rectangular pulses with the different frequencies and amplitudes, is able to achieve the desired multifocal pattern very accurately. For instance, as can be shown in Figures 6.5(a) and 6.5(c), the pressure profile presents a periodic response with frequency $f_{d}=f_{2}-f_{1}=$ $275-225=50 \mathrm{kHz}$ that results in a periodic focusing pattern with $T_{d}=1 / f_{d}=20$ $\mu \mathrm{s}$. Moreover, as can be observed, although the pressure profile presents two foci, the response is highly distorted and the foci do not achieve their target acoustic intensities. Analogously, Figures 6.5(b) and 6.5(d) represent the results of the three foci waveform, showing an even more distorted profile as a result of the more complex interference pattern among the three harmonic responses.

\section{Spatial resolution of the system}

Spatial resolution is an important parameter of every acoustic focusing system. In this case, the spatial resolution of the system is given by the length and width of the acoustic intensity focus, specified by the FLHM (Full-Length Half Maximum) and FWHM (Full-Width Half Maximum), respectively. The FLHM is measured along the perpendicular axis of the lens ( $z$-axis), while the FWHM is measured along the parallel axis to the lens ( $r$-axis). Both values are calculated from the measured steady 2D intensity map, $|p(f, r, z)|^{2}$, for each operating frequency. As discussed in the main manuscript, once the lens is manufactured, the resolution 

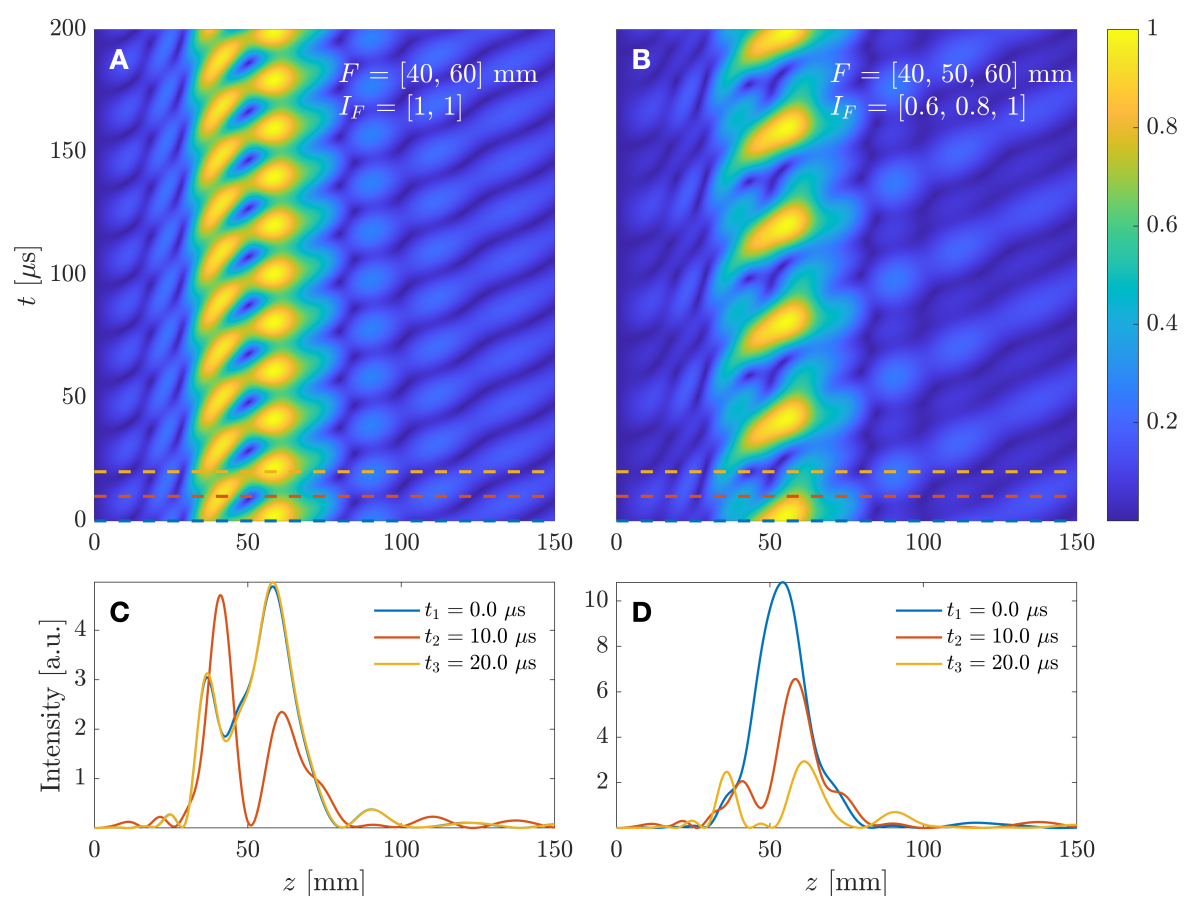

Figure 6.5: Continuous wave simulation results: $(\mathbf{A})$ pressure profile as a function of time for two equal intensity foci at 40 and $60 \mathrm{~mm}$, and (B) pressure profile for three foci at 50, 40, and $60 \mathrm{~mm}$ with normalized intensities $0.8,0.6$, and 1 , respectively. Panels (C) and (D) represent axial cuts at three different times for the two foci and three foci cases, respectively.

of the system at a given focal distance cannot be controlled individually with the operating frequency. If a higher resolution is required for a particular application, the size of the lens or the central frequency of the system should be increased. As can be observed from Figure 6.6, the measured lateral resolution remains almost constant at $3.5 \mathrm{~mm}$ in all the frequency range, while the axial resolution ranges from 12 to $18 \mathrm{~mm}$. However, in terms of the operating wavelength, the resolution decreases when the frequency increases. For the three considered focal distances in the main manuscript, $F=\{40,50,60\} \mathrm{mm}$, the measured lateral resolutions are $\mathrm{FWHM}=\{3.38,3.49,3.53\} \mathrm{mm}$ while the measured axial resolutions are $\mathrm{FLHM}=\{12.81,14.11,13.02\} \mathrm{mm}$, respectively. 

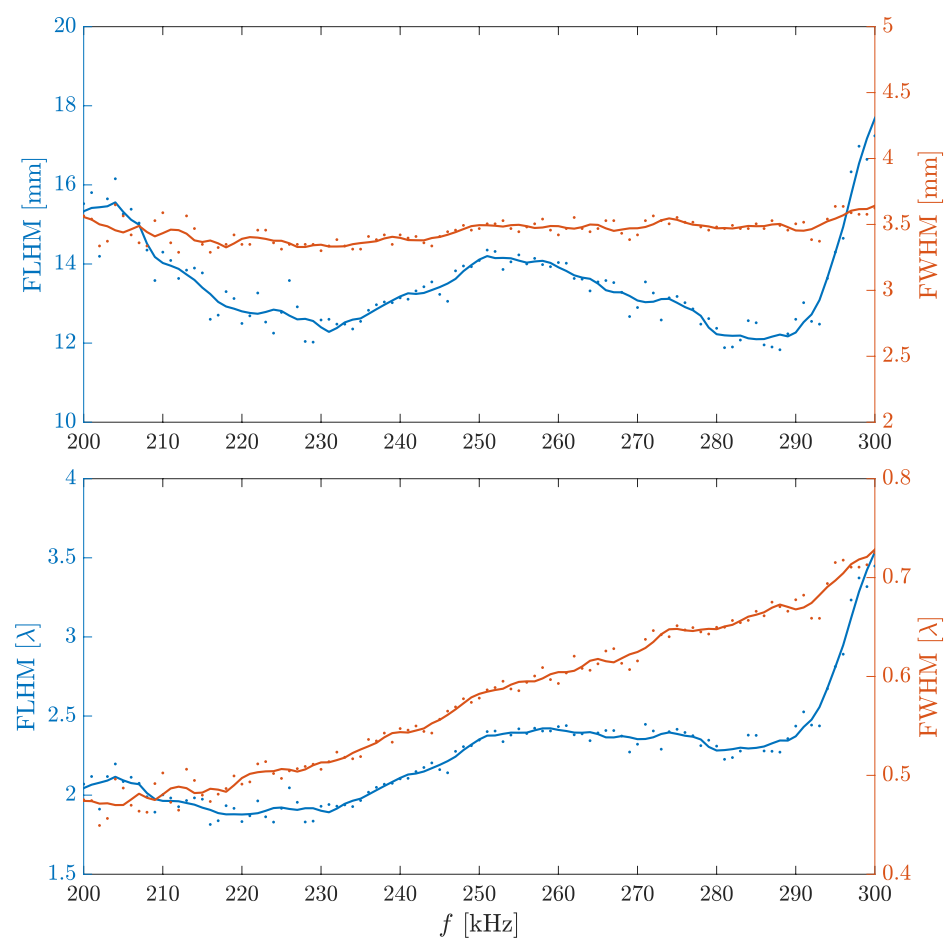

Figure 6.6: Measured axial (FLHM, left side) and lateral (FWHM, right side) resolutions as a function of the operating frequency. Top panel corresponds to absolute resolution values, while bottom panel corresponds to resolution values normalized to the operating wavelength.

\section{Experimental axial spectrum characterization}

The axial spectrum of the lens is measured using a three step process. First, the input waveform is designed and uploaded to the AWG directly using its associated MATLAB interface. Then, the axial profile is measured using the $3 \mathrm{D}$ automated positioning system described in the Materials and Methods section of the main manuscript, which provides the axial profile as a function of time. Finally, the axial spectrum is calculated as the Fourier transform of the measured time domain pressure profile, and then equalized using the spectrum of the input waveform to compensate its effect on the measured response, that is 


$$
p_{z}^{e q}(\omega, z)=\frac{p_{z}(\omega, z)}{|X(f)|}
$$

being $X(f)$ the spectrum of the input waveform.
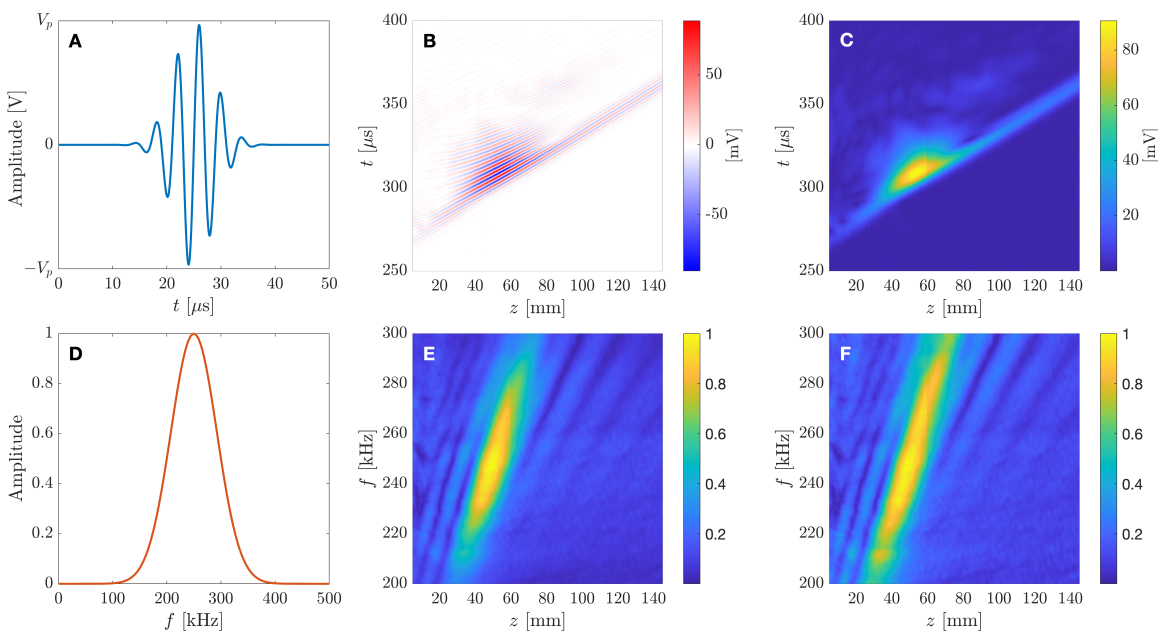

Figure 6.7: Axial spectrum characterization: (A) input modulated gaussian pulse used for the characterization and (D) its corresponding spectrum. (B) Measured bipolar pressure profile as a function of time and (C) absolute value of its complex Hilbert transform. (E) Absolute value of the measured pressure profile Fourier transform and (F) equalized axial spectrum of the lens.

The input pulse is designed to provide a gaussian spectrum centered at the central design frequency of the lens $\left(f_{0}=250 \mathrm{kHz}\right)$ with a bandwidth $(\Delta f)$ wide enough to characterize the lens response at the design frequencies. In this case the bandwidth is set to $\Delta f=100 \mathrm{kHz}$, which allows to measure the axial spectrum from 200 to $300 \mathrm{kHz}$. The pulse is calculated as

$$
x(t)=V_{p} \cdot \exp \left(-\frac{\left(t-t_{0}\right)^{2}}{2 \sigma_{t}^{2}}\right) \cdot \sin \left(2 \pi f_{0}\left(t-t_{0}\right)\right),
$$

where $V_{p}$ is the peak voltage of the waveform, $t_{0}$ is the time point when the pulse envelope reaches its maximum value, and $\sigma_{t}$ is the standard deviation of the pulse, given by 


$$
\sigma_{t}=\frac{\sqrt{\ln 4}}{\pi \Delta f}
$$

In this case, the pulse duration is set to $50 \mu \mathrm{s}$ and $t_{0}$ is set to $25 \mu \mathrm{s}$. Figure 6.7(a) shows the input waveform and Figure 6.7(d) depicts its corresponding spectrum. Figure 6.7 (b) represents the measured time domain bipolar pressure profile, while Figure 6.7 (c) depicts the absolute value of its complex Hilbert transform along the time axis. Finally, Figure 6.7(e) represents the absolute value of the Fourier transform of Figure 6.7(b), and 6.7(f) depicts the axial spectrum after equalizing Figure 6.7(e) using the spectrum shown in Figure 6.7(d). 
Chapter 6. Spatio-temporal Ultrasound Beam Modulation to Sequentially achieve Multiple Foci with a Single Planar Monofocal Lens 


\title{
Chapter 7
}

\section{Fractal Lenses based on Cantor Binary Sequences for Ultrasound Focusing Applications}

\author{
S. Pérez-López, J. M. Fuster, P. Candelas, and C. Rubio, "Fractal \\ lenses based on Cantor binary sequences for ultrasound focusing \\ applications, " Ultrasonics, vol. 99, p. 105967, Nov. 2019. (๑2019 \\ Elsevier B.V. \\ https://doi.org/10.1016/j.ultras.2019.105967
}

\section{Abstract}

In this work, we demonstrate the application of Cantor fractal lenses in acoustics. The Cantor Zone Plate (CZP), previously introduced in optics, is designed from a conventional Fresnel Zone Plate (FZP) using a binary sequence governed by the distribution of a generalized Cantor set. The CZP maintains its main focus at the same focal distance than its associated FZP, providing a softer multi-foci focusing profile which is very useful in certain ultrasound therapeutic applications. Experimental measurements are in good agreement with the theoretical model, demonstrating that CZPs are suitable for the ultrasound field. 


\subsection{Introduction}

Wave focusing is a hot research topic in many areas of physics, such as optics [1], microwaves [2], X-rays [3] or acoustics [4, 5]. Among the different types of lenses with focusing capabilities, Fresnel Zone Plates (FZPs) stand out for their easier fabrication process compared with traditional curved lenses. FZPs consist on a set of concentric rings, being each one of them a Fresnel region. The width of each ring depends on parameters such as the focal distance and the wavelength. There are two different types of zone plates (ZPs) depending on how the Fresnel regions are implemented. Soret ZPs [6] alternate transparent with opaque regions, while Rayleigh-Wood ZPs [7, 8] replace opaque areas with phase-reversal regions.

In the last years, new types of lenses based on the use of binary sequences have been proposed in optics, such as Fibonacci ZPs [9, 10], Cantor ZPs [11-13] and Thue-Morse ZPs [14, 15]. All these novel ZPs are variations over conventional FZPs, where transparent and opaque/phase-reversal Fresnel regions are distributed according to a specific binary sequence. Cantor ZPs (CZPs) are based on binary sequences that are governed by a fractal structure known as the generalized Cantor set, and they present a multifocal focusing profile with interesting self-similarity properties. They become appealing alternatives in therapeutic applications [16-19] where a softer tissue heating profile is desired.

Although alternative approaches in Cantor planar lenses design have been previously reported in ultrasounds [20,21], these designs are not based on FZPs and do not allow to control the zone plate focusing profile features. As an example, the location of the lens main focus is an unknown at the design stage and cannot be determined until these type of lenses are fully characterized after fabrication. However, with the design method provided in this work, we can accurately establish the location of the main focus at the design stage as in the conventional FZP case.

As it has been previously addressed in [22], the decision of whether selecting a transparent or an opaque central region in a FZP is a design parameter that becomes critical when directional transducers are employed. In this work, we show that this parameter also plays a significant role in the focusing enhancement of CZPs. 


\subsection{Theory}

As stated previously, CZPs are based on conventional FZPs, although a CZP uses a different criterion based on its governing binary sequence to determine whether a certain Fresnel region is either transparent or opaque/phase-reversal. In our setup, the distance between the transducer and the lens is not long enough to consider plane wave incidence, and therefore, the radii of the different Fresnel regions are calculated considering the spherical wave incidence equation:

$$
d+F+\frac{n \lambda}{2}=\sqrt{r_{n}^{2}+F^{2}}+\sqrt{r_{n}^{2}+d^{2}},
$$

where $F$ represents the focal distance, $d$ is the separation between the lens and the transducer, $\lambda$ is the wavelength, $r_{n}$ is the $n$-th Fresnel radius and $n=1,2, \ldots, N$, being $N$ the total number of Fresnel regions.

Once the different Fresnel radii have been calculated, the Fresnel regions are established. Then, different types of ZPs can be generated by stating which of the Fresnel regions become transparent areas or opaque/phase-reversal areas, using a binary sequence to provide this information. The binary sequence length is equal to the total number of Fresnel regions, and each position in the binary sequence maps to its corresponding Fresnel region. In Soret ZPs, type-1 and type- 0 elements of the binary sequence represent opaque and transparent regions, respectively, or vice versa. We define a O-ZP ("O-" stands for opaque) when type1 elements represent opaque sections, while a T-ZP ("T-" stands for transparent) relates type- 1 elements with transparent regions. Hence, O-ZPs present an opaque central region, while their T-ZPs counterparts present a transparent central region.

For instance, a periodic binary sequence of $N$ elements of alternating " 1 "s and " 0 "s produces a conventional FZP of $N$ Fresnel regions, as shown in Figures 7.1(a), 7.1(b) and 7.1(c). In this case, an O-FZP has been implemented, as type-1 elements represent opaque regions.

In order to generate a Cantor binary sequence, an initial sequence of $2 M-1$ elements, known as the generator, is created at the first stage $(S=1)$, with $M$ type- 1 elements and $M-1$ type- 0 elements, sorted with an alternating distribution. On the next stages, type-1 elements are replaced with replicas of the generator sequence, while each type- 0 element is replaced with a sequence of " 0 "s of the same length as the generator. The number of elements in the Cantor binary sequence at any stage can be calculated as $N=(2 M-1)^{S}$. Table 7.1 shows several examples of Cantor binary sequences for different values of $M$ and $S$, while table 7.2 shows a comparison between the binary sequences of three CZPs with $N=9, N=25$ and $N=27$ elements and their associated binary sequences 
(a)

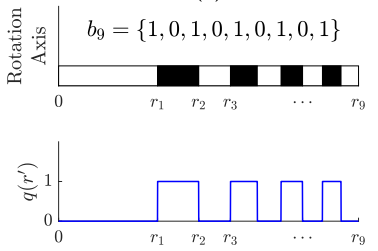

(d)

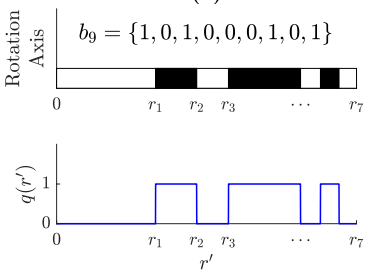

(b)

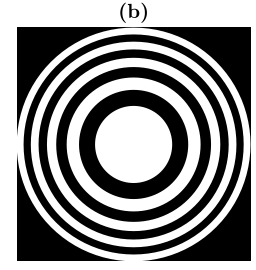

(e)

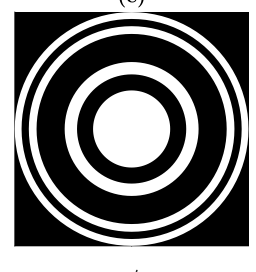

(c)

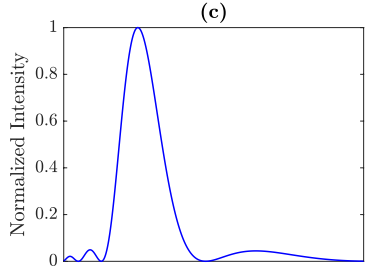

(f)

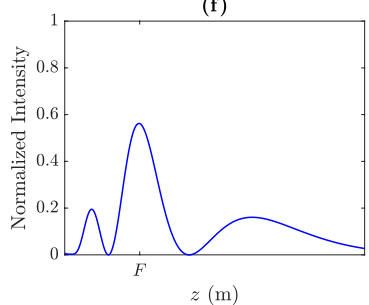

Figure 7.1: FZP design example with $N=9$ : (a) Periodic binary sequence and its corresponding pupil function, (b) FZP layout and (c) FZP focusing profile. CZP design example with $N=9$ : (d) Generalized Cantor sequence with $M=2$ and $S=2$ and its corresponding pupil function, (e) CZP layout, (f) CZP focusing profile. [ [`2019 Elsevier B.V.]

of conventional FZPs with the same number of regions. The associated FZP is always a periodic binary sequence of the same length than the original Cantor binary sequence.

\begin{tabular}{cclc}
\hline$M$ & $S$ & Cantor binary sequence & $N$ \\
\hline \hline & 1 & $\{101\}$ & 3 \\
2 & 2 & $\{101000101\}$ & 9 \\
& 3 & $\{1010001010000000000101000101\}$ & 27 \\
\hline \multirow{2}{*}{3} & 1 & $\{10101\}$ & 5 \\
& 2 & $\{1010100000101010000010101\}$ & 25 \\
\hline 4 & 1 & $\{1010101\}$ & 7 \\
\hline
\end{tabular}

Table 7.1: Cantor binary sequences for different combinations of $M$ and $S$.

Hence, once the different Fresnel regions of the conventional FZP are generated using equation 7.1, the Cantor binary sequence defines the lens pupil function which is used to generate the CZP, as can be observed in Figures 7.1(d), 7.1(e) and $7.1(\mathrm{f})$. The pupil function, $q\left(r^{\prime}\right)$, describes the geometry of the lens, indicating whether a specific Fresnel region is transparent or opaque to the ultrasound emission. In O-ZPs, type- 1 elements correspond to $q\left(r^{\prime}\right)=0$ (opaque regions), while type- 0 elements determine $q\left(r^{\prime}\right)=1$ (transparent regions). On the contrary, 


\begin{tabular}{ccl}
\hline$N$ & Zone Plate & Binary sequence \\
\hline \hline \multirow{2}{*}{9} & CZP & $\{101000101\}$ \\
& FZP & $\{101010101\}$ \\
\hline \multirow{2}{*}{25} & CZP & $\{1010100000101010000010101\}$ \\
& FZP & $\{1010101010101010101010101\}$ \\
\hline \multirow{2}{*}{27} & CZP & $\{101000101000000000101000101\}$ \\
& FZP & $\{101010101010101010101010101\}$ \\
\hline
\end{tabular}

Table 7.2: Binary sequences for different CZPs and their associated FZPs.

in T-ZPs, type- 1 and type- 0 elements correspond to $q\left(r^{\prime}\right)=1$ (transparent) and $q\left(r^{\prime}\right)=0$ (opaque), respectively. Thus, both the FZP and the CZP shown in Figure 1 correspond to O-ZPs. As can be observed from Figures 7.1(c) and 7.1(f), the distribution of the acoustic intensity along the focusing profile is different for FZPs and CZPs. While a FZP concentrates the acoustic intensity at a single focal point, the CZP distributes its focusing profile among three different foci, a main focus at the same location than that of the associated FZP, and two additional foci with lower intensities that soften the overall focusing profile and can be very appealing in certain therapeutic applications. Thus, the acoustic intensity is distributed differently in both focusing profiles.

To analyze the focusing properties of a CZP, or in general of any $\mathrm{ZP}$, the acoustic intensity generated by the lens can be numerically calculated using the Rayleigh-Sommerfeld diffraction integral [23]:

$$
I(r, z)=\frac{1}{2 \rho_{0} c_{0} \lambda^{2}}\left|\int_{0}^{2 \pi} \int_{0}^{+\infty} q\left(r^{\prime}\right) p_{i}\left(r^{\prime}\right) \frac{r^{\prime}}{R} \cos (n, R) e^{-j k R} d r^{\prime} d \varphi\right|^{2},
$$

where $k=2 \pi / \lambda$ is the wavenumber, $r$ is the radial axis, $z$ is the longitudinal axis, $r^{\prime}$ is the radial axis at the surface of the lens, $\varphi$ is the rotation angle at the surface of the lens, $p_{i}\left(r^{\prime}\right)$ is the incident pressure at the lens, $q\left(r^{\prime}\right)$ is the pupil function, $R=\sqrt{z^{2}+r^{2}+\left(r^{\prime}\right)^{2}-2 r r^{\prime} \cos \varphi}$ and $\cos (n, R)=z / R$, being $n$ the normal direction to the lens surface. $\rho_{0}$ and $c_{0}$ are the density and sound speed of the host medium, respectively, which in this case is water.

In the plane wave incidence case, $p_{i}\left(r^{\prime}\right)$ is constant and does not affect the integral result. However, in the ultrasound case, a piston transducer is employed as an emitter, affecting the lens focusing profile [22]. In the far field, the piston transducer can be modelled as a point source emitter with a given directivity 
pattern:

$$
p_{i}\left(r^{\prime}\right)=\frac{j k p_{0} a^{2}}{2 \sqrt{\left(r^{\prime}\right)^{2}+d^{2}}} D\left(r^{\prime}\right) e^{-j k \sqrt{\left(r^{\prime}\right)^{2}+d^{2}}},
$$

with $a$ being the piston active radius, $p_{0}$ the pressure at the surface of the piston and $D\left(r^{\prime}\right)$ the piston directivity pattern, which is given by

$$
D\left(r^{\prime}\right)=\frac{2 J_{1}(k a \sin \theta)}{k a \sin \theta},
$$

where $J_{1}$ is the first kind and first order Bessel function and $\theta=\arctan \left(r^{\prime} / d\right)$ is the angle referred to the normal direction of the surface of the piston.

(a)

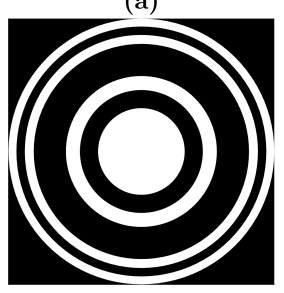

(c)

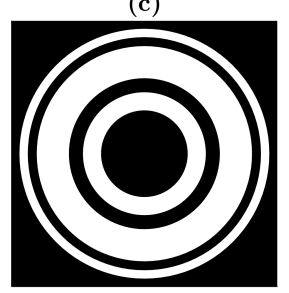

(b)

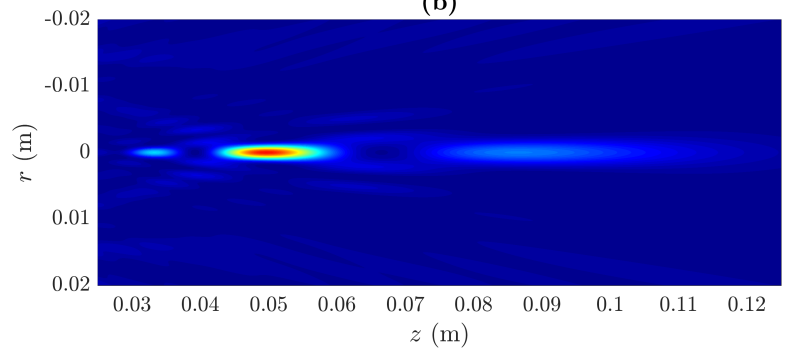

(d)

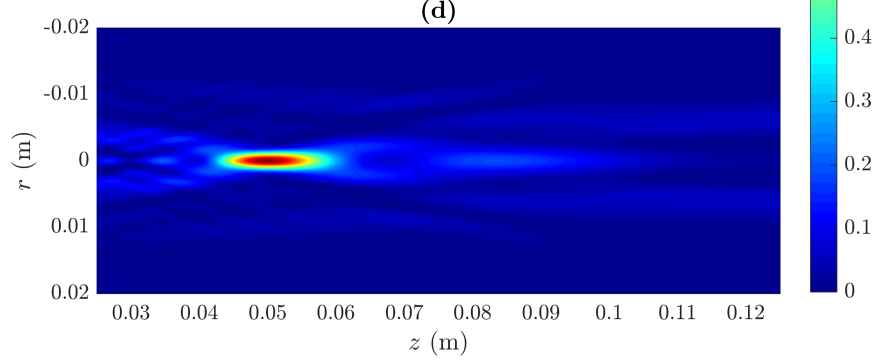

Figure 7.2: Numerical results: (a) O-CZP and (b) its computed intensity map, (c) T-CZP and (d) its computed intensity map. [`2019 Elsevier B.V.]

Figure 7.2 shows simulated normalized intensity maps for two different CZPs. Intensity maps have been computed using equation 7.2 , considering a piston transducer with $12.7 \mathrm{~mm}$ of active diameter. Both CZPs are designed with $M=2$ and $S=2$, a focal distance of $F=50 \mathrm{~mm}$, a working frequency of $1 \mathrm{MHz}$ and a transducer separation distance of $d=340 \mathrm{~mm}$. The difference between both CZPs is that the first one has been designed considering a central pressure blocking region (O-CZP) while the second one has been designed considering a central transparent region (T-CZP). As can be observed from Figures 7.2(b) and 7.2(d), the T-CZP (Figure 7.2(c)) achieves a higher intensity level at the 
focal distance compared to the O-CZP (Figure 7.2(a)). This phenomenon is a consequence of the directional behaviour of the piston transducer, as its main energy contribution is generated at the perpendicular direction of the transducer surface. Thus, in the O-CZP case, the main energy contribution is reflected back at the central blocking region, whereas in the T-CZP case, it fully contributes to the focal intensity, enhancing the CZP focusing capability. Both CZP intensity maps have been normalized to the maximum value for comparison purposes, which corresponds to the T-CZP case.

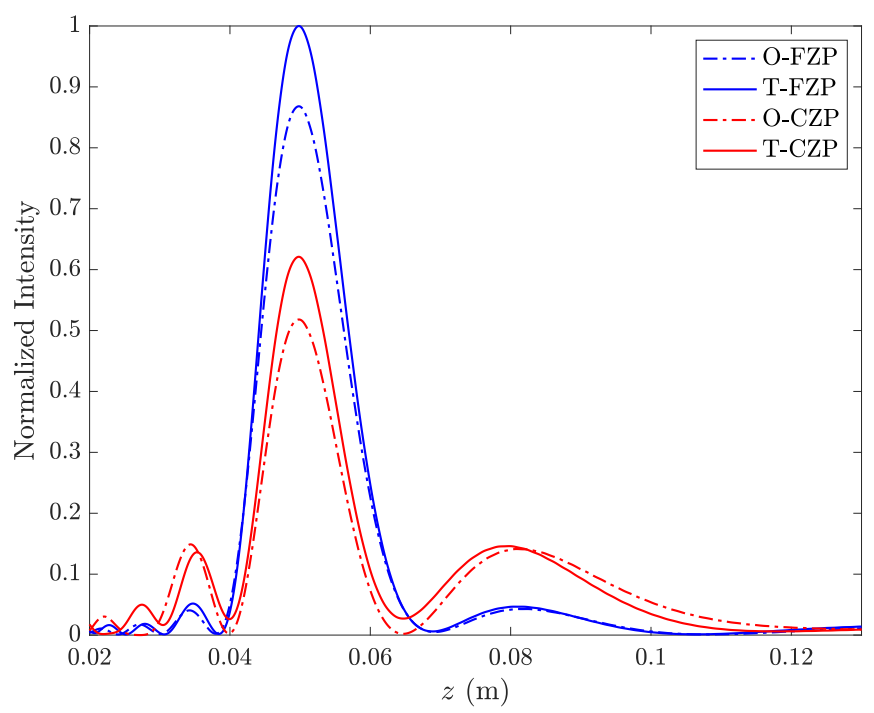

Figure 7.3: Focusing profiles for O-CZP (red dashed line), T-CZP (red solid line) and their associated O-FZP (blue dashed line) and T-FZP (blue solid line). In all cases, $F=50 \mathrm{~mm}, d=340 \mathrm{~mm}$ and $\lambda=1.5 \mathrm{~mm}$. [ [`2019 Elsevier B.V.]

Figure 7.3 depicts the focusing profiles of the acoustic intensity maps shown in Figure 7.2 compared to the focusing profiles of their associated FZPs with the same focal distance and for both transparent ( $\mathrm{T}_{-}$) and opaque ( $\left.\mathrm{O}_{-}\right)$central regions. All focusing profiles have been normalized to the maximum intensity value, which corresponds to the T-FZP case. As can be observed from Figure 7.3, both FZP lenses achieve higher focal intensities than their corresponding CZPs, because CZPs distribute the incident energy among their secondary foci. Moreover, Figure 7.3 also shows that lenses with transparent central regions (T-FZP and T-CZP) achieve a higher focal intensity than their associated counterparts with opaque central regions (O-FZP and O-CZP), as a consequence of the directional ultrasound emission of the transducer. However, it is worth noting that although the T-CZP 
reaches a higher intensity value than the O-CZP, the T-CZP presents a more distorted focusing profile. This distortion translates into a small focal displacement and intensity reduction of the secondary foci, which are slightly shifted towards the main focus and provide lower relative intensity levels when compared to the $\mathrm{O}-\mathrm{CZP}$ focusing profile. Although this distortion is very low, there is a trade-off between focal intensity level and focusing profile distortion when selecting T-CZPs over O-CZPs.

\subsection{Experimental Results}

Experimental measurements have been carried out to demonstrate that CZPs are suitable for ultrasound focusing applications. Figure 7.4 shows the experimental set-up, which consists of a 3D underwater automated positioning system with a spatial resolution of $1 \times 1 \times 1 \mathrm{~mm}^{3}$ and three degrees of freedom. The signal is generated using a Panametrics 5077PR pulser and then, it is transmitted using a piston transducer from Imasonic with a $12.7 \mathrm{~mm}$ active diameter and a central frequency of $1 \mathrm{MHz}$. A needle hydrophone from Precision Acoustics with a diameter of $1.5 \mathrm{~mm}$ is used as receiver, and its output signal is amplified using a low noise preamplifier and then digitized, using a digital oscilloscope from Pico Technology.

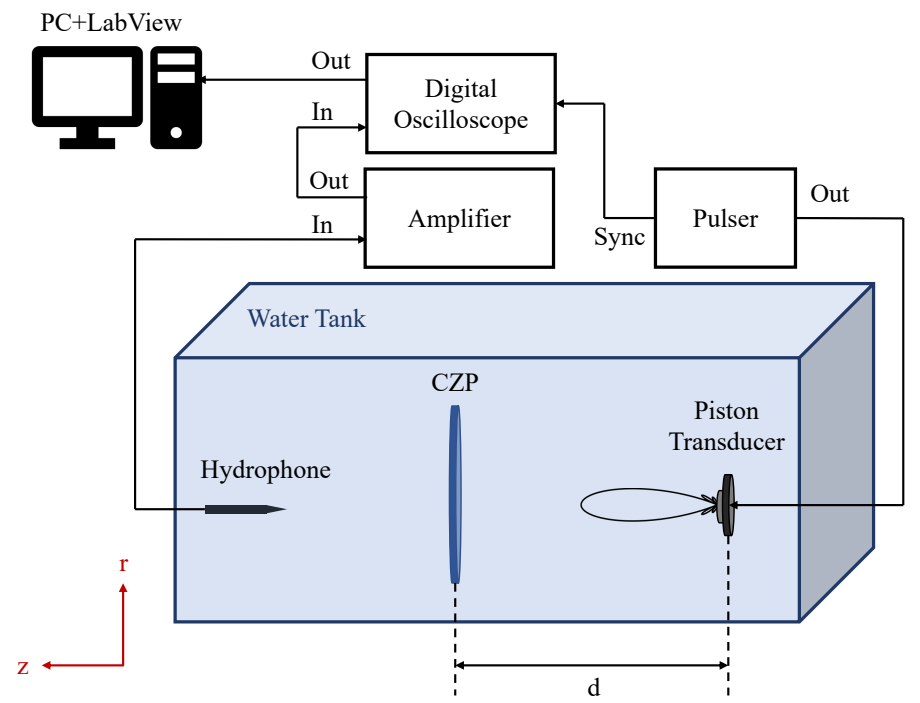

Figure 7.4: Schematic view of the experimental set-up. [`2019 Elsevier B.V.] 
Two Soret CZPs have been manufactured using brass for implementing the opaque Fresnel regions, because this material presents a high acoustic impedance contrast with water. Both CZPs have been designed with $M=2$ and $S=2$ for an operating frequency of $1 \mathrm{MHz}(\lambda=1.5 \mathrm{~mm})$, a focal distance $F=50 \mathrm{~mm}$ and a separation between the lens and the transducer of $d=340 \mathrm{~mm}$. Figure 7.5(a) shows a O-CZP (blocking central region), whereas Figure 7.5(b) shows a T-CZP (transparent central region). The outermost radius of the O-CZP is $r_{N}=24.87$ $\mathrm{mm}$, whereas for the T-CZP is $r_{N}=23.38 \mathrm{~mm}$. Thus, the T-CZP presents a smaller size compared to the O-CZP because the O-CZP implements one additional Fresnel opaque region. Figures 7.5(c) and 7.5(d) show the corresponding measured intensity maps. Both measured intensity maps are normalized to the higher value, which corresponds to the T-CZP case, following the same procedure as previously in the simulation. As it can be observed from Figure 7.5, T-CZP achieves a higher focal intensity level than O-CZP, which agrees with numerical results shown in Figure 7.2.

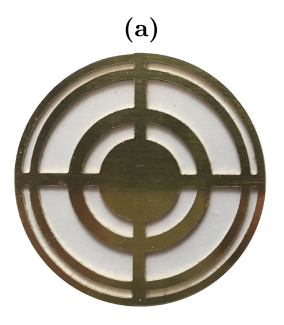

(b)

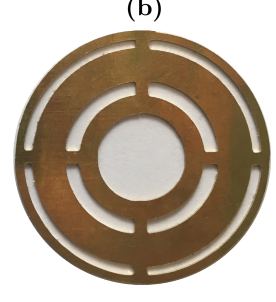

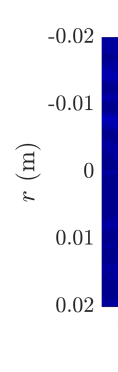

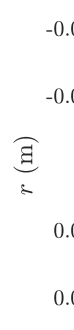

(c)

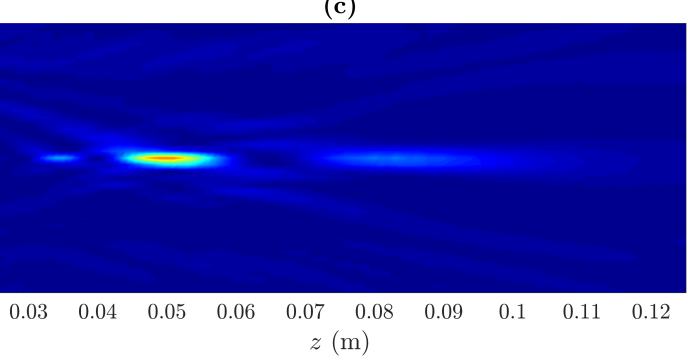

(d)

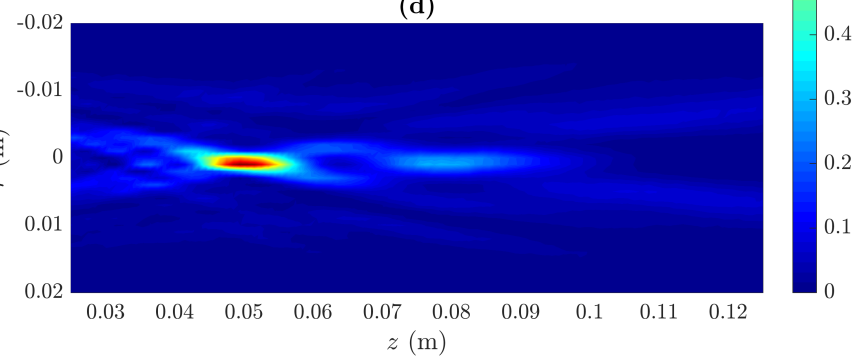

Figure 7.5: Experimental results: (a) O-CZP and (c) its measured intensity map, (b) T-CZP and (d) its measured intensity map. [`2019 Elsevier B.V.]

Figure 7.6 shows the longitudinal and transversal cuts corresponding to both O-CZP and T-CZP. Blue solid lines represent experimental results, while red dashed lines correspond to numerical results. Figures 7.6(a) and 7.6(b) depict axial longitudinal cuts, also known as focusing profiles, against the axial coordinate. The results show good agreement between simulations and experimental 
(a)

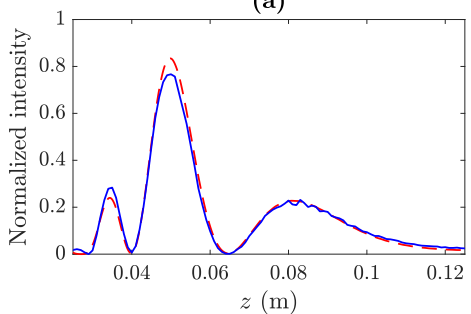

(c)

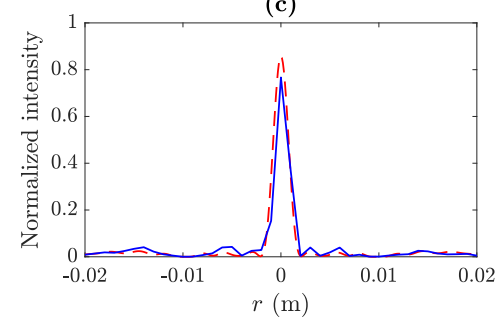

(b)

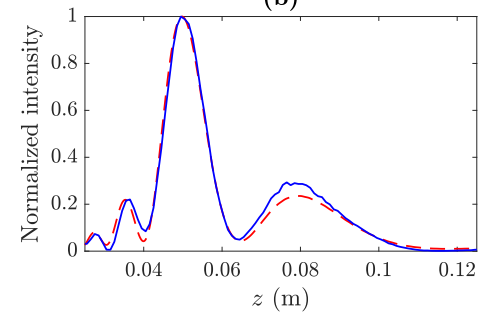

(d)

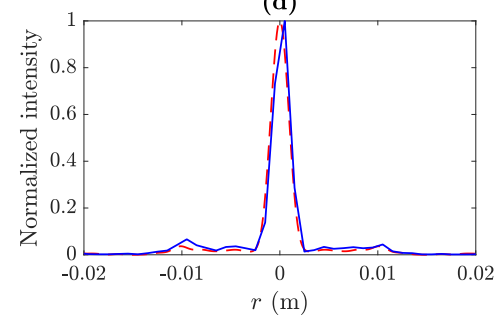

Figure 7.6: O-CZP: (a) focusing profile and (c) transversal cut at the focal plane. T-CZP: (b) focusing profile and (d) transversal cut at the focal plane. Blue solid lines represent experimental measurements and red dashed lines correspond to numerical results. [`2019 Elsevier B.V.]

measurements. The triple-foci focusing profile, which corresponds to $M=2$ and $S=2$, has potential applications in therapeutic treatments where a softer progressive heating profile is required. Experimental measurements and simulation results match very well at the main lobe of both lenses, which present Full Length Half Maximum (FLHM) values of $11.48 \mathrm{~mm}$ for O-CZP and $11.39 \mathrm{~mm}$ for T-CZP (Table 7.3), being both foci located at the designed focal length of $50 \mathrm{~mm}$. The secondary foci of the O-CZP are located at 35 and $81 \mathrm{~mm}$, while the secondary foci of the T-CZP are located closer to the main focus at 36 and $77 \mathrm{~mm}$, which agrees with the results depicted in Figure 7.3. However, slight differences between simulations and experiments can be appreciated at the focusing profile secondary lobes. These small differences are mainly due to the cross-shaped mechanical support of the manufactured CZPs that can be observed in Figures 7.5(a) and 7.5(c), which maintain the FZP brass rings fixed in place. This cross-shaped structure has not been considered in the simulation. Figures 7.6(c) and 7.6(d) depict the transversal cuts at the focal distance against the radial coordinate for O-CZP and $\mathrm{T}-\mathrm{CZP}$, respectively. Both simulation and experimental results agree again very well, showing transversal profiles with negligible side lobes and with a narrow main lobe which presents Full Width Half Maximum (FWHM) values of $1.59 \mathrm{~mm}$ in the O-CZP case and $2.09 \mathrm{~mm}$ in the T-CZP case, as shown in Table 7.3. This absence 
of secondary lobes in the transversal cuts is due to the piston transducer influence, as the amplitude of the incident pressure at the lens in this case has a similar effect to FZP apodization through decreasing diffraction efficiency regions [24].

Table 7.3 shows, as stated before, the measured FLHM and FWHM for both CZP lenses, but also provides the measured normalized intensity at the focus, which becomes a very significant parameter, and demonstrates that when operating in underwater ultrasound with piston emitters, the choice of whether the central Fresnel region is transparent or opaque is critical [22]. The T-CZP option (transparent central Fresnel region) results in a $30.43 \%$ enhancement at the focal point intensity over the O-CZP option (opaque central Fresnel region). This significant improvement in the focusing capabilities of the CZP lens is due to the use of directional piston transducers, which favors the use of central transparent regions.

\begin{tabular}{lcccc}
\hline & $F(\mathrm{~mm})$ & FLHM $(\mathrm{mm})$ & FWHM $(\mathrm{mm})$ & $I_{F}$ \\
\hline \hline O-CZP & 50 & 11.48 & 1.59 & $76.67 \%$ \\
T-CZP & 50 & 11.39 & 2.09 & $100 \%$ \\
\hline
\end{tabular}

Table 7.3: Measured F, FLHM, FWHM and normalized focal intensity $\left(I_{F}\right)$ values for O-CZP and T-CZP.

Figure 7.7 depicts the focusing profiles of the manufactured CZPs at three different frequencies: $f=0.9 \mathrm{MHz}$ (blue lines), $f=1.0 \mathrm{MHz}$ (black lines), which corresponds to the design frequency, and $f=1.1 \mathrm{MHz}$ (red lines). Figures 7.7(a) and 7.7(b) correspond to the O-CZP and T-CZP cases, respectively. Numerical results are depicted with dashed lines, whereas experimental measurements are depicted with solid lines. As can be observed from Figure 7.7, the modification of the operating frequency shifts the lens focal distance. Both O-CZP and TCZP shift their focal distance to $F=56.35 \mathrm{~mm}$ when the operating frequency is increased to $f=1.1 \mathrm{MHz}$. In contrast, when the frequency is reduced to $f=0.9$ $\mathrm{MHz}$, the focal distance is shifted to $F=43.46 \mathrm{~mm}$. Moreover, the secondary foci of the CZP profile are also shifted similarly with the operating frequency. Figure 7.7 illustrates that the magnitude of the shift is related to the current focal length. Thus, the secondary focus that is farther from the lens is the focus that presents a higher shift when the operating frequency is modified. Hence, the operating frequency becomes an additional parameter very appealing to dynamically control the focal distance of CZP lenses. 
(a)

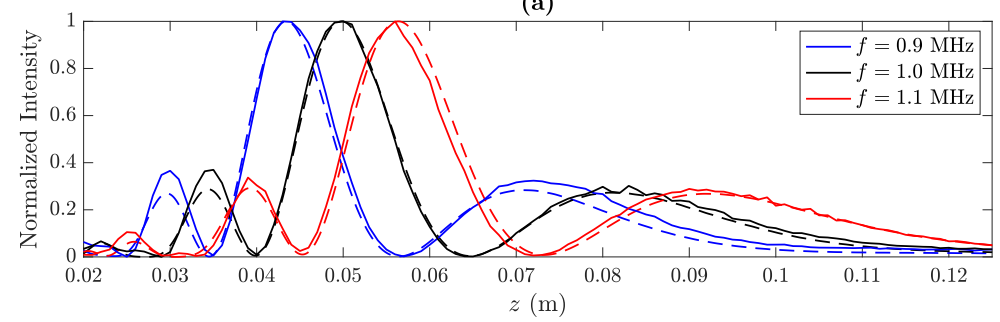

(b)

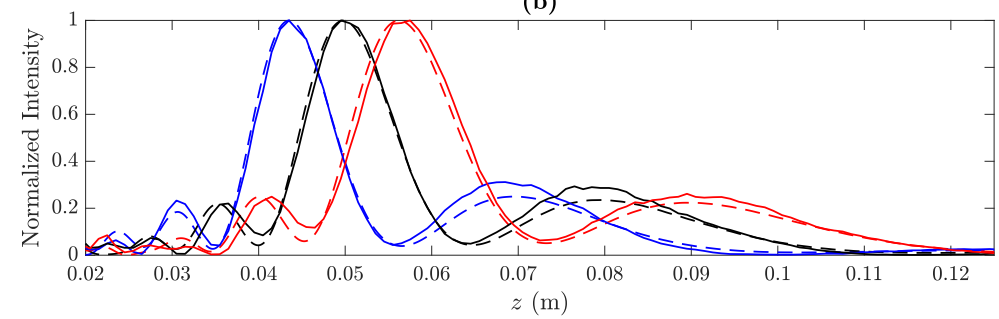

Figure 7.7: Focusing profile for manufactured CZPs at different frequencies: (a) O-CZP and (b) T-CZP. All profiles are normalized to their maximum values. Solid lines represent experimental measurements, while dashed lines represent numerical results. [` 2019 Elsevier B.V.]

\subsection{Conclusion}

In this work, a theoretical analysis on the design and experimental characterization of Cantor Zone Plates have been carried out. These lenses produce focusing profiles with their main focus at the same location than their associated FZPs and multiple secondary lobes. Experimental results for two manufactured CZPs have been presented, showing good agreement with the theoretical model. Results show that when using directional piston emitters in underwater transmission, CZPs designed with central transparent regions (T-CZPs) achieve higher focal intensity levels than those designed with central pressure blocking regions (O-CZPs). It has been experimentally demonstrated that the measured focal intensity of the T-CZP case provides a $30.43 \%$ enhancement of the intensity level compared to the O-CZP case. Thus, this work shows that CZPs become appealing alternatives in ultrasound focusing applications, such as therapeutic treatments which require a softer heating profile. 


\section{References}

[1] R. S. Rodrigues Ribeiro, P. Dahal, A. Guerreiro, P. A. S. Jorge, and J. Viegas, "Fabrication of Fresnel plates on optical fibres by FIB milling for optical trapping, manipulation and detection of single cells," Scientific Reports, vol. 7, p. 4485 , dec 2017.

[2] H. Hristov and M. Herben, "Millimeter-wave Fresnel-zone plate lens and antenna," IEEE Transactions on Microwave Theory and Techniques, vol. 43, no. 12, pp. 2779-2785, 1995.

[3] K. Jefimovs, O. Bunk, F. Pfeiffer, D. Grolimund, J. van der Veen, and C. David, "Fabrication of Fresnel zone plates for hard X-rays," Microelectronic Engineering, vol. 84, pp. 1467-1470, may 2007.

[4] D. C. Calvo, A. L. Thangawng, M. Nicholas, and C. N. Layman, "Thin Fresnel zone plate lenses for focusing underwater sound," Applied Physics Letters, vol. 107, p. 014103, jul 2015.

[5] J. M. Fuster, P. Candelas, S. Castiñeira-lbáñez, S. Pérez-López, and C. Rubio, "Analysis of fresnel zone plates focusing dependence on operating frequency," Sensors (Switzerland), vol. 17, p. 2809, dec 2017.

[6] J. L. Soret, "Ueber die durch Kreisgitter erzeugten Diffractionsphänomene," Annalen der Physik und Chemie, vol. 232, no. 9, pp. 99-113, 1875.

[7] L. Rayleigh, Wave Theory, vol. 24. 1888.

[8] J. Kirz, "Phase zone plates for $x$ rays and the extreme uv," Journal of the Optical Society of America, vol. 64, p. 301, mar 1974.

[9] J. A. Monsoriu, A. Calatayud, L. Remon, W. D. Furlan, G. Saavedra, and P. Andres, "Bifocal Fibonacci Diffractive Lenses," IEEE Photonics Journal, vol. 5, pp. 3400106-3400106, jun 2013.

[10] F. Machado, V. Ferrando, W. D. Furlan, and J. A. Monsoriu, "Diffractive m-bonacci lenses," Optics Express, vol. 25, p. 8267, apr 2017.

[11] G. Saavedra, W. D. Furlan, and J. A. Monsoriu, "Fractal zone plates," Optics Letters, vol. 28, p. 971, jun 2003.

[12] W. D. Furlan, G. Saavedra, and J. A. Monsoriu, "White-light imaging with fractal zone plates," Optics Letters, vol. 32, no. 15, p. 2109, 2007.

[13] O. Mendoza-Yero, M. Fernández-Alonso, G. Mínguez-Vega, J. Lancis, V. Climent, and J. A. Monsoriu, "Fractal generalized zone plates," Journal of the Optical Society of America A, vol. 26, p. 1161, may 2009. 
[14] V. Ferrando, F. Giménez, W. D. Furlan, and J. A. Monsoriu, "Bifractal focusing and imaging properties of Thue-Morse Zone Plates," Optics Express, vol. 23, p. 19846, jul 2015.

[15] T. Xia, S. Cheng, and S. Tao, "Generation of three equal-intensity foci based on a modified composite zone plate," Optik, vol. 159, pp. 150-156, apr 2018.

[16] G. ter Haar and C. Coussios, "High intensity focused ultrasound: Physical principles and devices," International Journal of Hyperthermia, vol. 23, pp. 89-104, jan 2007.

[17] D. Suo, Z. Jin, X. Jiang, P. A. Dayton, and Y. Jing, "Microbubble mediated dual-frequency high intensity focused ultrasound thrombolysis: An In vitro study," Applied Physics Letters, vol. 110, p. 023703, jan 2017.

[18] S. GyP, L. DY, and H. G, "What is on the Horizon for Hyperthermic Cancer Therapy?," Journal of Traditional Medicine \& Clinical Naturopathy, vol. 06, no. $02,2017$.

[19] J. C. Simon, O. A. Sapozhnikov, V. A. Khokhlova, Y.-N. Wang, L. A. Crum, and M. R. Bailey, "Ultrasonic atomization of tissue and its role in tissue fractionation by high intensity focused ultrasound," Physics in Medicine and Biology, vol. 57, pp. 8061-8078, dec 2012.

[20] S. Castiñeira-Ibáñez, D. Tarrazó-Serrano, J. Fuster, P. Candelas, and C. Rubio, "Polyadic Cantor Fractal Ultrasonic Lenses: Design and Characterization," Applied Sciences, vol. 8, p. 1389, aug 2018.

[21] D. Tarrazó-Serrano, S. Castiñeira-lbáñez, P. Candelas, J. M. Fuster, and C. Rubio, "Numerical simulation and laboratory measurements on the influence of fractal dimension on the acoustic beam modulation of a Polyadic Cantor Fractal lenses," Applied Acoustics, vol. 148, pp. 119-122, may 2019.

[22] S. Pérez-López, J. M. Fuster, P. Candelas, and C. Rubio, "On the focusing enhancement of Soret zone plates with ultrasound directional transducers," Applied Physics Letters, vol. 114, p. 224101, jun 2019.

[23] J. W. Goodman, Introduction to Fourier optics. Roberts and Company Publishers, 2005.

[24] A. Takeuchi, K. Uesugi, and Y. Suzuki, "Improvement of quantitative performance of imaging $\mathrm{x}$-ray microscope by reduction of edge-enhancement effect," Journal of Physics: Conference Series, vol. 849, p. 012055, jun 2017. 


\section{Chapter 8}

\section{M-bonacci Zone Plates for Ultrasound Focusing}

S. Pérez-López, J. M. Fuster, and P. Candelas, "M-Bonacci Zone Plates for Ultrasound Focusing," Sensors, vol. 19, no. 19, p. 4313, Oct. 2019.

https://doi.org/10.3390/s19194313

\section{Abstract}

In this work, we present a thorough analysis on M-bonacci zone plates for ultrasound focusing applications. These planar lenses are capable of providing bifocal focusing profiles with equal intensity in both foci, and become very appealing for a wide range of applications including medical and industrial focusing. We show that in long wavelength domains, such as acoustics or microwaves, the separation between both foci can be finely adjusted at the expense of slightly increasing the distortion of the focusing profile, and we introduce a design parameter to deal with this issue and simplify the design process of these lenses. Experimental measurements are in good agreement with numerical simulations and demonstrate the potential of M-bonacci lenses in ultrasound focusing applications. 


\subsection{Introduction}

Acoustic lenses are devices capable of focusing incident sound waves into specific focal areas. Due to their wide range of applications, including both industrial and medical, acoustic lenses are a hot topic among the scientific community. Nowadays, approaches to focus acoustic waves are mainly based on metasurfaces, which can be implemented with subwavelength slits [1], coiling-up space structures [2-4] or Helmholtz resonators [5]. Holographic structures have also been proposed as a flexible solution for synthesis of focusing profiles [6]. However, these kind of devices are usually difficult to design because they require a complete 3D design of each their unit cells. Other focusing alternatives are based on either spherical [7] or cylindrical [8] containers filled with liquids, where the acoustic properties of the inner liquid determines the lens focusing profile. Although these type of lenses can be very interesting in certain applications, and they have shown focal tunability [7], they can only focus in very close-range applications, which limits their potential. In this sense, a simpler device capable of focusing acoustic waves with higher flexibility is the Fresnel Zone Plate (FZP), which can be implemented with either alternating blocking or absorbing regions with transparent regions [9, $10]$, or alternating transparent with phase-reversal regions [11].

FZPs have been widely used in many areas of physics due to their advantageous planar fabrication compared to conventional curved lenses. A FZP focuses waves through constructive interference of the diffracted field in its apertures. These devices can be found in all sorts of applications, such as optical trapping [12], planar antenna design [13] or ultrasound focusing applications $[9,14,10]$. In optics, the distance between the transducer and the lens is usually large enough to consider plane wave incidence. However, in many ultrasound and microwave applications, a directional emitter is placed at a distance where the plane wave approximation is not valid. Therefore, in order to properly obtain the focusing profile of an acoustic lens, the influence of the transducer on the lens energy distribution has to be considered. $[10,15]$

In the past years, several lenses with interesting focusing properties have been introduced in the optical domain, such as Fibonacci ZPs [16, 17], Cantor ZPs [18-20] and Thue-Morse ZPs [21, 22]. All these novel ZPs are variations over conventional FZPs, where transparent and opaque/phase-reversal Fresnel regions are distributed according to a specific binary sequence [23]. M-bonacci Zone Plates (MbZPs) [17] are based on M-bonacci sequences, a generalization of the well known Fibonacci sequence. They produce bifocal focusing profiles with equal intensity focus at distances related to the M-bonacci sequence ratio. The application of ultrasound focusing techniques is a topic of great interest in both photo-acoustic imaging [24, 25] and therapeutic applications [26-29]. In 
the latter, dual focusing profiles can be used to either treat two isolated regions simultaneously or target extensive areas with higher efficiency [30, 31].

Although M-bonacci sequences have been previously proposed for ultrasound focusing applications [23], in this work we experimentally demonstrate the feasibility of MbZPs in the ultrasound domain for the first time. Moreover, we analyze the distortion introduced in the focusing profile by these type of lenses in long wavelength domains, such as acoustics or microwaves, when the Fresnel approximation is not fulfilled, introducing a new parameter which becomes very helpful at the design stage and allows a fine adjustment of the separation between both foci at the expense of slightly distorting the MbZP focusing profile.

\subsection{M-bonacci Zone Plates}

\subsubsection{Design and Focusing Properties}

MbZPs are formed by aperiodic binary sequences based on M-bonacci series. To build a M-bonacci serie of order $m$ and stage $j$, two initiators of the sequence are defined as in the Fibonacci case, $F_{m, 1}=0$ and $F_{m, 2}=1$. The next numbers of the series are calculated as

$$
F_{m, j+1}=\left\{\begin{array}{cl}
\sum_{i=1}^{j} F_{m, i} & , j \leq m \\
\sum_{i=j-m+1}^{j} F_{m, i} & , j>m
\end{array},\right.
$$

which means that the next numbers of the series are calculated as the sum of the $m$-previous numbers.

Table 8.1 shows the first ten numbers of the M-bonacci series calculated for $m=2$ (Fibonacci case), $m=3$ and $m=4$.

\begin{tabular}{c|l}
\hline$m$ & $F_{m, j}$ \\
\hline 2 & $\{0,1,1,2,3,5,8,13,21,34\}$ \\
3 & $\{0,1,1,2,4,7,13,24,44,81\}$ \\
4 & $\{0,1,1,2,4,8,15,29,56,108\}$ \\
\hline
\end{tabular}

Table 8.1: M-bonacci sequences for different $m$ values.

One interesting parameter that can be derived from the M-bonacci series is 
the sequence ratio, $\varphi_{m}$, defined as the limit of the ratio between two consecutive numbers:

$$
\varphi_{m}=\lim _{j \rightarrow+\infty}\left(\frac{F_{m, j+1}}{F_{m, j}}\right) .
$$

When $m=2$, the ratio corresponds to the golden mean $\varphi_{2}=1.618$, while for $m=3$ and $m=4$ the sequence ratios are $\varphi_{3}=1.839$ and $\varphi_{4}=1.928$, respectively.

Analogously to the M-bonacci series, a binary M-bonacci sequence can be formed using the initiators $f_{m, 1}=0$ and $f_{m, 2}=1$. The next instance of the Mbonacci sequence is obtained as the concatenation of the $m$-previous M-bonacci instances, using the following equation,

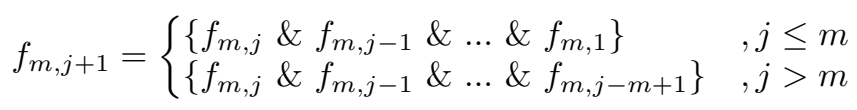

where "\&" represents the concatenation operator.

\begin{tabular}{l|lll}
\hline$j$ & $m=2$ & $m=3$ & $m=4$ \\
\hline 1 & $\{0\}$ & $\{0\}$ & $\{0\}$ \\
2 & $\{1\}$ & $\{1\}$ & $\{1\}$ \\
3 & $\{10\}$ & $\{10\}$ & $\{10\}$ \\
4 & $\{101\}$ & $\{1010\}$ & $\{1010\}$ \\
5 & $\{10110\}$ & $\{1010101\}$ & $\{10101010\}$ \\
6 & $\{10110101\}$ & $\{1010101101010\}$ & $\{101010101010101\}$ \\
\hline
\end{tabular}

Table 8.2: Examples of M-bonacci binary sequences.

Table 8.2 shows the first six iterations of M-bonacci binary sequences for $m=2, m=3$ and $m=4$. In each sequence, there are $F_{m, j+1}$ binary elements, being $F_{m, j}$ type- 1 and $F_{m, j+1}-F_{m, j}$ type- 0 . The notation regarding type- 1 and type- 0 elements is used to indicate whether the binary element is either a 1 or a 0 , and thus, determines how the corresponding Fresnel region is implemented. In Soret ZPs, type- 1 elements are implemented as opaque regions, whereas type- 0 elements correspond to transparent regions. As an example, the binary sequence $f_{2,6}=\{10110101\}$, corresponding to $m=2$ and $j=6$, indicates that the first, third, fourth, sixth and eight Fresnel regions of the lens are opaque regions (implemented in brass), while the rest (second, fifth and seventh regions) are transparent. The number of opaque (type-1), transparent (type-0) and total Fresnel regions can also be verified using the numbers of the M-bonacci series shown in Table 8.1, resulting for this particular example in a total of $F_{m, j+1}=F_{2,7}=8$ regions, with 
$F_{2,6}=5$ type- 1 opaque regions and $F_{2,7}-F_{2,6}=3$ type- 0 transparent regions. The limit of the ratio between the number of type- 1 and type- 0 elements is given by

$$
\tau_{m}=\lim _{j \rightarrow+\infty}\left(\frac{F_{m, j}}{F_{m, j+1}-F_{m, j}}\right)=\frac{1}{\varphi_{m}-1} .
$$

As stated before, M-bonacci ZPs are obtained by applying a binary sequence to a conventional FZP. Once the M-bonacci binary sequence has been calculated, the MbZP is obtained assigning type- 1 and type- 0 elements to the corresponding Fresnel regions of its associated conventional FZP. When plane wave incidence is considered, the radii of the different Fresnel regions are given by

$$
r_{n}=\sqrt{n \lambda z_{0}+\left(\frac{n \lambda}{2}\right)^{2}}
$$

where $\lambda$ represents the wavelength, $z_{0}$ the focal distance, and $n=1,2, \ldots, N$, being $N$ the total number of Fresnel regions.

(a)

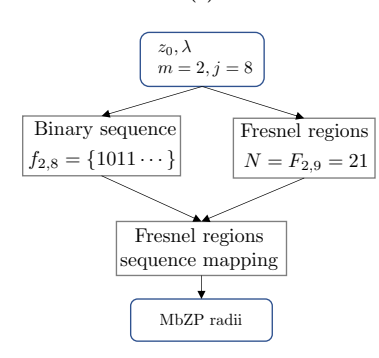

(b)

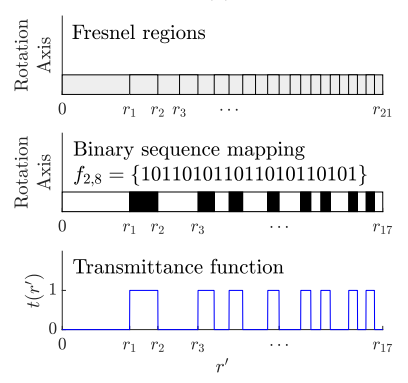

(c)

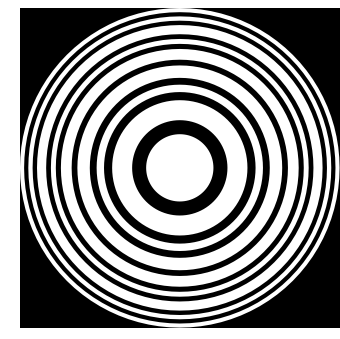

Figure 8.1: Lens design procedure with $m=2$ and $j=8$ : (a) design steps; (b) Fresnel regions (top), clasification of MbZP regions after the binary sequence mapping (middle) and final transmittance function of the lens (bottom); (c) resulting MbZP layout.

Figure 8.1 shows a MbZP design example with $m=2$ and $j=8$. The flow diagram of the lens design procedure is depicted in Figure 8.1(a). Figure 8.1(b) shows the distribution of the Fresnel regions for the current example and their mapping using the M-bonacci sequence distribution. Finally, Figure 8.1(c) depicts the final MbZP layout resulting from the lens design.

Once the M-bonacci $Z P$ radii are obtained, the focusing profile of the lens can be calculated by numerically computing the Rayleigh-Sommerfeld diffraction 
integral,

$$
I(z)=\frac{4 \pi^{2}}{\lambda^{2}}\left|\int_{0}^{a} p_{i}\left(r^{\prime}\right) t\left(r^{\prime}\right) \frac{e^{-j k r}}{r} \cos (n, r) r^{\prime} d r^{\prime}\right|^{2},
$$

where $a$ is the maximum radius of the lens, $r^{\prime}$ is the radial coordinate of the lens, $k=2 \pi / \lambda$ is the wavenumber, $p_{i}\left(r^{\prime}\right)$ is the incident pressure distribution, $t\left(r^{\prime}\right)$ is the ZP transmittance function and $r=\sqrt{\left(r^{\prime}\right)^{2}+z^{2}}, z$ is the axial coordinate and $\cos (n, r)=z / r$, being $n$ the normal direction to the lens surface. For a Soret $\mathrm{ZP}$, the transmittance function, also known as pupil function, is 0 at the pressure blocking regions and 1 at the transparent regions.

In equation 8.6, the distance $r$ can be expressed as

$$
r=z \sqrt{1+\left(\frac{r^{\prime}}{z}\right)^{2}}
$$

Using the Taylor expansion $\sqrt{1+x} \cong 1+x / 2-x^{2} / 8, r$ can be approximated as

$$
r \cong z+\frac{\left(r^{\prime}\right)^{2}}{2 z}-\frac{\left(r^{\prime}\right)^{4}}{8 z^{3}}
$$

The Fresnel approximation assumes that the third term of the Taylor expansion of $r$ does not affect the exponential result, which means that its contribution to the exponent has to be much lower than $2 \pi$, that is,

$$
k \frac{\left(r^{\prime}\right)^{4}}{8 z^{3}}<<2 \pi \text {. }
$$

The worst case is obtained when $r^{\prime}$ reaches its maximum value at $r^{\prime}=a$, which means that the Fresnel approximation stands for axial coordinates greater than

$$
z^{3}>>\frac{a^{4}}{8 \lambda}
$$

If the above condition is met, $r \cong z+\frac{\left(r^{\prime}\right)^{2}}{2 z}$, and equation 8.6 can be approximated as

$$
I(z) \cong \frac{4 \pi^{2}}{\lambda^{2}}\left|\int_{0}^{a} p_{i}\left(r^{\prime}\right) t\left(r^{\prime}\right) \frac{e^{-j k z} e^{-j k \frac{\left(r^{\prime}\right)^{2}}{2 z}}}{z+\frac{\left(r^{\prime}\right)^{2}}{2 z}} \cos (n, r) r^{\prime} d r^{\prime}\right|^{2} .
$$

Further approximations can be done, as the paraxial approximation is valid $(z>>$ $\left.r^{\prime}\right)$ and therefore $\cos (n, r) \cong 1$ and the denominator at the integral becomes $z+\frac{\left(r^{\prime}\right)^{2}}{2 z} \cong z$, which results in

$$
I(z) \cong \frac{4 \pi^{2}}{\lambda^{2} z^{2}}\left|\int_{0}^{a} p_{i}\left(r^{\prime}\right) t\left(r^{\prime}\right) e^{-j k \frac{\left(r^{\prime}\right)^{2}}{2 z}} r^{\prime} d r^{\prime}\right|^{2}
$$


It is convenient to implement a change of variable in both axial and radial directions. Thus, a variable $u=\frac{a^{2}}{2 \lambda}$ is defined as the normalized axial coordinate, whereas a variable $\xi=\left(\frac{r^{\prime}}{a}\right)^{2}$ is defined as the normalized radial coordinate. Keeping in mind that $d r^{\prime}=\frac{a}{2 \sqrt{\xi}} d \xi$, equation 8.12 becomes

$$
I(u) \cong 4 \pi^{2} u^{2}\left|\int_{0}^{1} p_{i}(\xi) t(\xi) e^{-j 2 \pi \xi u} d \xi\right|^{2},
$$

which means that if the Fresnel approximation condition is fulfilled (equation 8.10 is verified) the focusing profile of the lens can be obtained as a Fourier transform of the pressure distribution at the ZP aperture. When the Fresnel approximation is not applicable and equation 8.13 cannot be used, the focusing profile of the MbZP is computed using equation 8.6 and presents a certain distortion compared to the ideal case.

For a standard FZP, the focal distance in the normalized axial coordinate can be calculated using equation 8.5 as

$$
u_{0}=\frac{a^{2}}{2 \lambda z_{0}}=\frac{N \lambda z_{0}+\left(\frac{N \lambda}{2}\right)^{2}}{2 \lambda z_{0}}=\frac{N}{2}+\frac{N^{2} \lambda}{8 z_{0}}=\frac{N}{2}(1+\varepsilon),
$$

being $a=r_{N}$ the maximum radius of the lens and $\varepsilon=\frac{N \lambda}{4 z_{0}}$.

For low wavelength domains, such as optics, $\varepsilon<<1$ and therefore equation 8.14 can be approximated as

$$
u_{0} \cong \frac{N}{2} .
$$

Interestingly, as reported by Monsoriu et al. [16, 17], the focusing profile of MbZPs shows two symmetric foci respect to the normalized focal distance of its associated FZP $\left(u_{0}=N / 2=F_{m, j+1} / 2\right)$. The first focus is located at $u_{1} \cong F_{m, j}$, which corresponds to the number of type- 1 elements in the binary sequence, whereas the second focus is located at $u_{2} \cong F_{m, j+1}-F_{m, j}$, which corresponds to the number of type- 0 elements in the sequence. Therefore, the ratio between the two foci tends to $u_{1} / u_{2} \cong \tau_{m}$, as stated in equation 8.4. The focal positions in the denormalized axial coordinate can be calculated as

$$
z_{1}=\frac{F_{m, j+1}}{F_{m, j}} \cdot \frac{z_{0}}{2} \cong \varphi_{m} \frac{z_{0}}{2}
$$

and

$$
z_{2}=\frac{F_{m, j+1}}{F_{m, j+1}-F_{m, j}} \cdot \frac{z_{0}}{2} \cong \varphi_{m} \tau_{m} \frac{z_{0}}{2}
$$


(a)

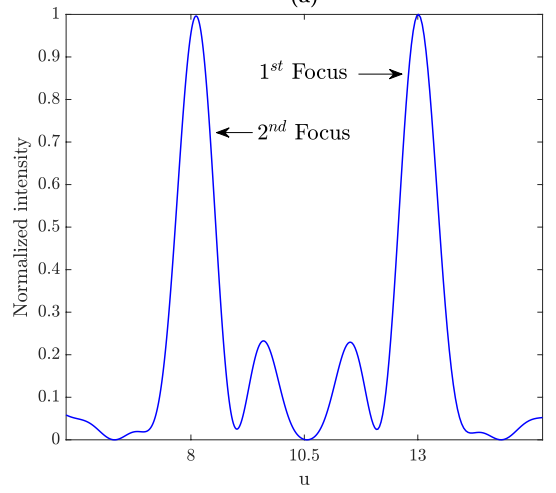

(b)

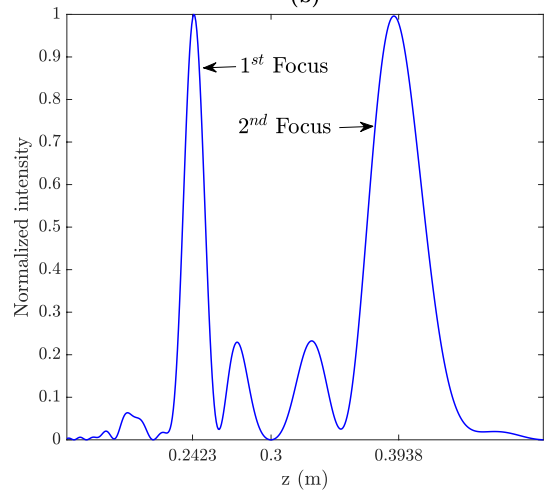

Figure 8.2: Focusing profile of a lens with $m=2, j=8, z_{0}=0.3 \mathrm{~m}$ and $\lambda=0.3$ $\mathrm{mm}$ as a function of: (a) the normalized axial coordinate and (b) the denormalized axial coordinate.

being $z_{0}$ the focal distance of the associated FZP.

Figures 8.2(a) and 8.2(b) show the focusing profile of a MbZP lens against the normalized $(u)$ and denormalized $(z)$ axial coordinate, respectively. As can be observed from Figure 8.2(a), when using the normalized axial coordinate, the MbZP is completely symmetrical as it was previously shown in optics [16]. The normalized focal distances are $u_{1} \cong F_{2,8}=13$ and $u_{2} \cong F_{2,9}-F_{2,8}=8$, which agrees with their theoretical values. On the other hand, when the axial coordinate is denormalized and the real focusing profile is depicted (Figure 8.2(b)), the foci are not symmetrical anymore, due to the coordinate transformation that relates $u$ with $z$. The main effect over the focusing profile is that the focus further from the lens becomes wider than the focus closer to the lens.

Figure 8.3 depicts the computed focusing profiles along the axial coordinate for three different MbZPs. Figure 8.3(a) corresponds to $m=2$, while 8.3(b) and 8.3(c) correspond to $m=3$ and $m=4$, respectively. As can be observed from Figure $8.3(\mathrm{a}), z_{1} \cong 81 \mathrm{~mm}$ and $z_{2} \cong 131 \mathrm{~mm}$, which agree with their theoretical focal distances obtained from equations 8.16 and 8.17. Figure 8.3(b) shows two foci at $z_{1} \cong 92 \mathrm{~mm}$ and $z_{2} \cong 110 \mathrm{~mm}$, while 8.3 (c) shows two foci at $z_{1} \cong 96$ $\mathrm{mm}$ and $z_{2} \cong 104 \mathrm{~mm}$, which also agrees with the theory. 
(a)

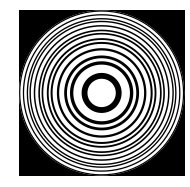

(b)

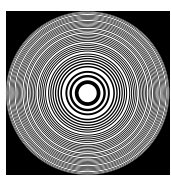

(c)

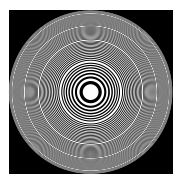

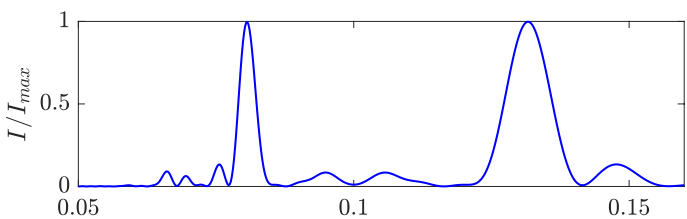
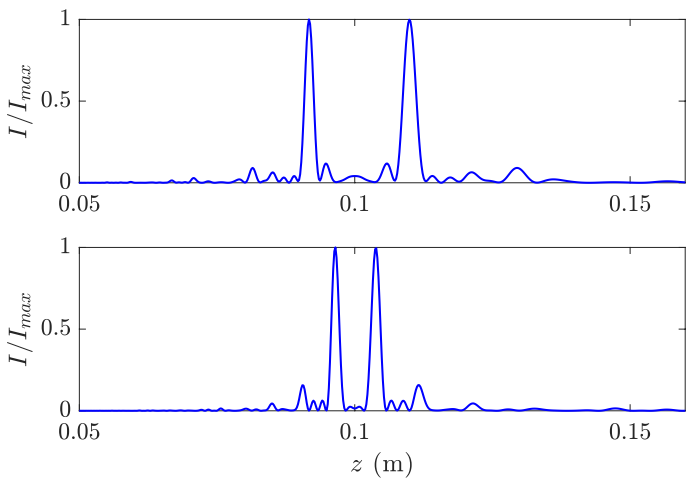

Figure 8.3: MbZP layouts (left) and their normalized focusing profiles (right): (a) $m=2$, (b) $m=3$ and (c) $m=4$. For all MbZPs, $j=9$ and $z_{0}=0.1 \mathrm{~m}$.

\subsection{2 $\gamma$-Parameter}

In long wavelength domains, such as ultrasounds, the Fresnel approximation can not always be assumed in typical focusing applications. Therefore, it is useful to define a new $\gamma$ parameter as the ratio between the associated FZP focal distance, $z_{0}$, and the Fresnel approximation distance, $z_{f}$, given by

$$
\gamma=\frac{z_{0}}{z_{f}}
$$

where $z_{f}=\sqrt[3]{\frac{a^{4}}{8 \lambda}}$

Thus, the Fresnel approximation is valid when $\gamma>>1$. In long wavelength domains, fulfilling this condition requires very long focal distances, which is not the usual case in most near-field focusing applications. However, simulations show that MbZPs present low distortion for $\gamma>2$ values, which is a more reasonable condition to achieve. In fact, MbZPs can be designed in the $1<\gamma<2$ range with low distortion in the focusing profile. Alternatively, if $\gamma<1 \mathrm{MbZPs}$ present a highly distorted focusing profile. This distortion results in focal displacement and focal intensity reduction compared to the $\gamma>>1$ case. Figure 8.4 depicts the numerically computed axial intensities using equation 8.6 for MbZPs (blue 

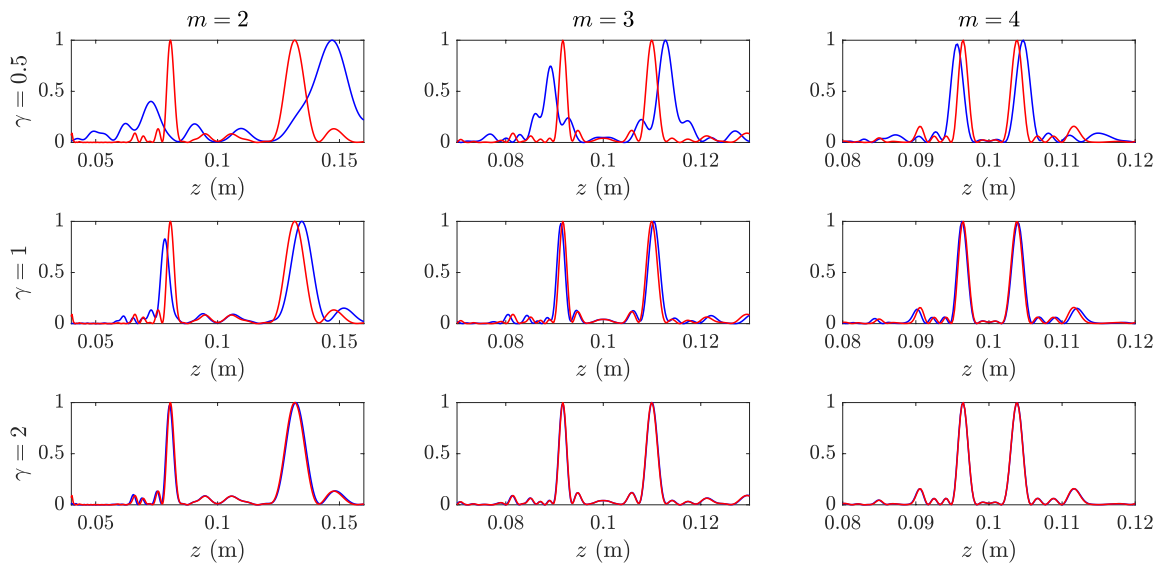

Figure 8.4: Focusing profiles (blue lines) against axial distance for different values of $\gamma$ (rows) and $m$ (columns), compared to their ideal counterparts $(\gamma>>1)$ in red.

lines) with different $\gamma$ and $m$ values, compared to the case when $\gamma>>1$ and the Fresnel approximation is valid (red lines). As can be observed from Figure 8.4 , when $\gamma=0.5$ (first row) the distortion is very severe, and the focal distances in all three cases do not correspond to the theoretical values. When $\gamma=1$, the distortion is significantly reduced in comparison with the $\gamma=0.5$ case, and the focusing profiles are closer to the theoretical value. Finally, when $\gamma=2$ (third row) minimum distortion is observed.

Thus, the $\gamma$ parameter should be considered in the design process of MbZPs. Combining equation 8.5 with the Fresnel approximation distance condition results in

$$
z_{f}^{3}=\frac{\left(F_{m, j+1} z_{0}\right)^{2}}{8} \lambda(1+\varepsilon)^{2}
$$

Using equations 8.18 and 8.19 and substituting $\varepsilon=\frac{N \lambda}{4 z_{0}}$ yields to the following third order equation:

$$
\frac{1}{16} \gamma^{3} F_{m, j+1}^{4} \psi^{3}+\frac{1}{2} \gamma^{3} F_{m, j+1}^{3} \psi^{2}+\gamma^{3} F_{m, j+1}^{2} \psi-8=0,
$$

where $\psi=\frac{\lambda}{z_{0}}$ is an additional design variable.

Hence, once $\psi$ has been numerically calculated solving equation 8.20 in order to achieve a specific $\gamma$ parameter value, it is possible to obtain the focal 
distance for a given wavelength, or the required wavelength for a specific focal distance.

(a)

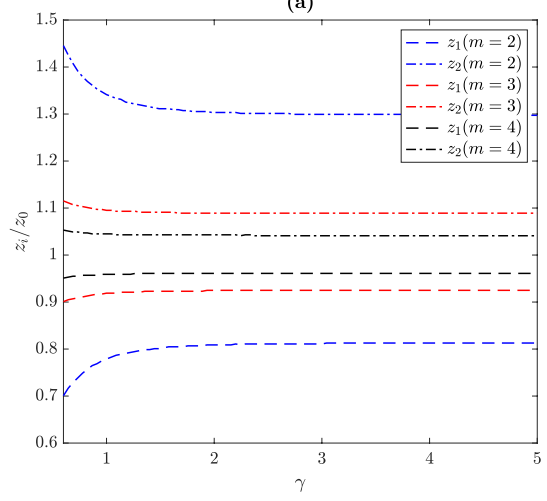

(b)

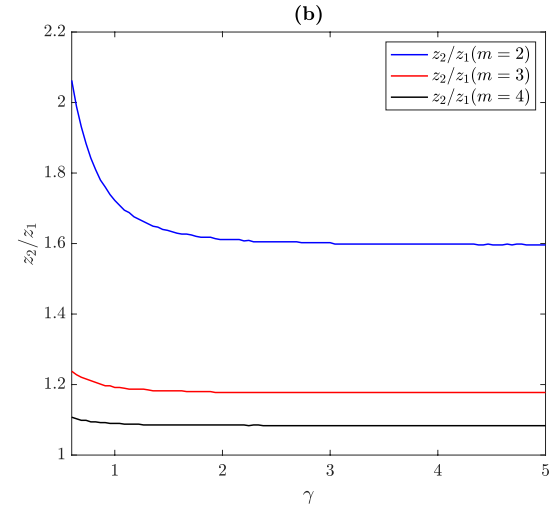

Figure 8.5: (a) Normalized focal distances and (b) focal distance ratio as a function of the $\gamma$ parameter.

Figure 8.5 (a) depicts the normalized focal distances for the first and second focus against the $\gamma$ parameter for three different cases: $m=2$ (red lines), $m=3$ (blue lines) and $m=4$ (black lines), as well as the focal distance ratio (Figure 8.5 (b)), defined as $z_{2} / z_{1}$, for the same three cases. The results have been obtained using equation 8.6. All MbZPs have been designed for a fixed $z_{0}$, while the wavelength has been adjusted using equation 8.20 in each simulation in order to provide the desired $\gamma$ factor. As can be observed from Figure 8.5, for $\gamma>2$ the focal distances of both foci tend to their theoretical positions $(\gamma \rightarrow+\infty$ case), whereas $\gamma<2$ results in both foci being shifted away in opposite directions. This phenomenon can be easily understood by observing the focal distance ratio, which theoretically tends to $z_{2} / z_{1} \cong \tau_{m}$, according to equations 8.16 and 8.17 . Thus, as the $\gamma$ parameter augments, $z_{2} / z_{1}$ tends to $1.60,1.18$ and 1.08 for $m=2, m=3$ and $m=4$, respectively, which are very close to the theoretical values given by $\tau_{2}=1.618, \tau_{3}=1.192$ and $\tau_{4}=1.078$. Moreover, when $\gamma<2$, the displacement on the focal distances is more noticeable in the $m=2$ case, which agrees with the results depicted in Figure 8.4.

Figure 8.6 illustrates a MbZP design example using the tools developed in this work. Figure 8.6(a) shows the concept diagram with the different steps required to accurately design the MbZP. Initially, the input parameters are the desired location of both foci, $z_{1}$ and $z_{2}$, and the resolution level of the lens, that is related with the MbZP size, and thus, with the $j$ parameter. In this particular example, these three parameters have been selected as $z_{1}=50 \mathrm{~mm}$, $z_{2}=90 \mathrm{~mm}$, and $j=8$. In the next design step, both the order of the $\mathrm{M}$ - 
(a)

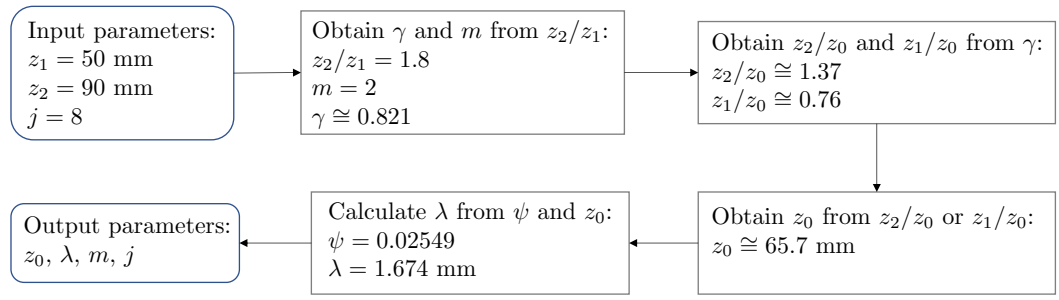

(b)

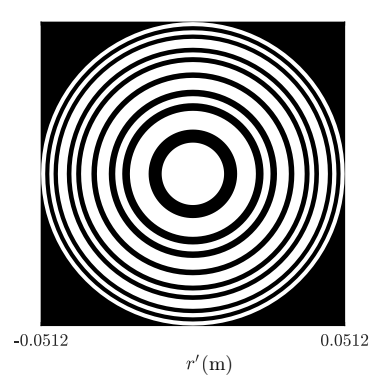

(c)

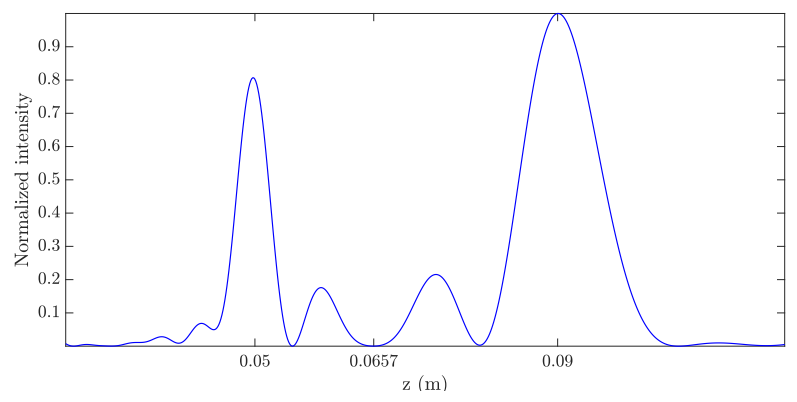

Figure 8.6: Lens design example with $z_{1}=50 \mathrm{~mm}$ and $z_{2}=90 \mathrm{~mm}$ : (a) Design procedure flow diagram, (b) resulting MbZP lens and (c) numerically computed focusing profile.

bonacci sequence $m$ and the required $\gamma$ parameter are found by computing the focal distance ratio, $z_{2} / z_{1}$, and then using Figure $8.5(\mathrm{~b})$ to retrieve them. With $m$ and $\gamma$, the normalized focal distances, $z_{1} / z_{0}$ and $z_{2} / z_{0}$, can be obtained using Figure 8.5(a), and therefore, $z_{0}$ is found. Using equation 8.20, the $\psi$ parameter can be found, and $\lambda$ is finally obtained as the product $z_{0} \psi$ to determine the design frequency for the MbZP lens.

Once the design parameters $m, j, \lambda$ and $z_{0}$ have been determined, the $\mathrm{MbZP}$ radii can be calculated as it was previously shown in Figure 8.1(a), and the MbZP lens can be built. Figure 8.6(b) shows the resulting MbZP layout for this particular design example in which $m=2, j=8, \lambda=1.67 \mathrm{~mm}$ and $z_{0}=65.7$ $\mathrm{mm}$, and Figure 8.6(c) depicts the MbZP focusing profile with the first and second foci located at positions $z_{1}=50 \mathrm{~mm}$ and $z_{2}=90 \mathrm{~mm}$ as required, demonstrating the feasibility and utility of this design method. 


\subsection{Experimental Results and Discussion}

(a)

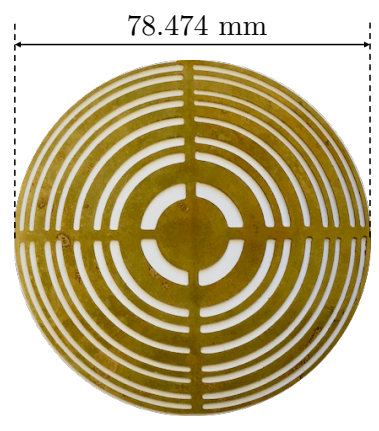

(b)

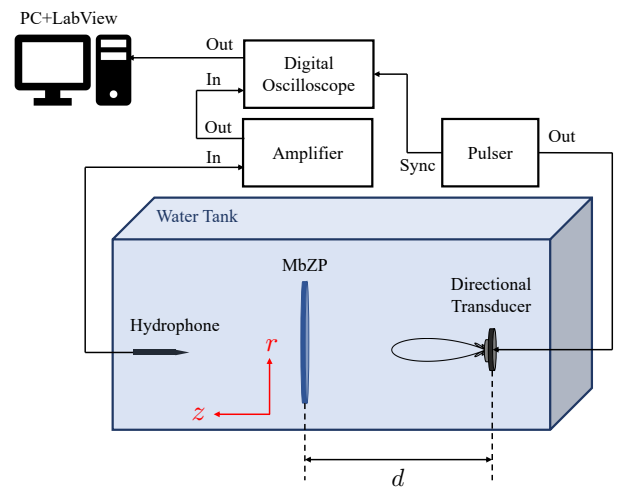

Figure 8.7: (a) Manufactured MbZP and (b) scheme of the experimental set-up.

Experimental measurements have been carried out in order to validate the theoretical analysis. The experimental set-up consists of an underwater 3D automated positioning system with a spatial resolution of $1 \times 1 \times 1 \mathrm{~mm}^{3}$. An Imasonic piston transducer with $12.7 \mathrm{~mm}$ of active diameter and a central working frequency of $1 \mathrm{MHz}$ is used as emitter. A needle hydrophone form Precision Acoustics Ltd. with $1.5 \mathrm{~mm}$ of diameter and a $-4 \mathrm{~dB}$ bandwidth ranging from $200 \mathrm{kHz}$ to 25 $\mathrm{MHz}$ is used as receiver. The transmitted signal is generated using a Panametrics 5077PR pulser and sampled using a digital oscilloscope from Pico Technology with a resolution of 12-bit. Figure 8.7 (b) depicts a scheme of the experimental set-up.

Since plane wave incidence can not be assumed due to the reduced physical dimensions of the water tank, the Fresnel radii were calculated using the spherical wave incidence equation, given by

$$
d+z_{0}+\frac{n \lambda}{2}=\sqrt{d^{2}+r_{n}^{2}}+\sqrt{z_{0}^{2}+r_{n}^{2}},
$$

where $d=350 \mathrm{~mm}$ is the transducer separation from the lens. In order to obtain the $\gamma$ parameter under spherical wave incidence, an iterative process is used. In this process, the $\psi$ value for plane wave incidence is used to obtain an initial value of $\lambda$. Subsequently, the wavelength is iteratively increased until the desired $\gamma$ value is achieved.

A Soret MbZP, made of brass and designed with $m=2, j=8, z_{0}=50$ 
$\mathrm{mm}$ and $\lambda=1.5 \mathrm{~mm}$, has been manufactured (Figure 8.7(a)). In this lens, the Fresnel approximation distance is $z_{f}=58.24 \mathrm{~mm}$ and therefore, $\gamma=0.859$, meaning that the focusing profile of the lens is going to be significantly distorted. Figure 8.8(a) depicts the measured acoustic intensity map, whereas 8.8(b) shows the measured focusing profile (squares) along the axial distance compared to the FEM simulation (blue line) and the $\gamma>>1$ case (red line).
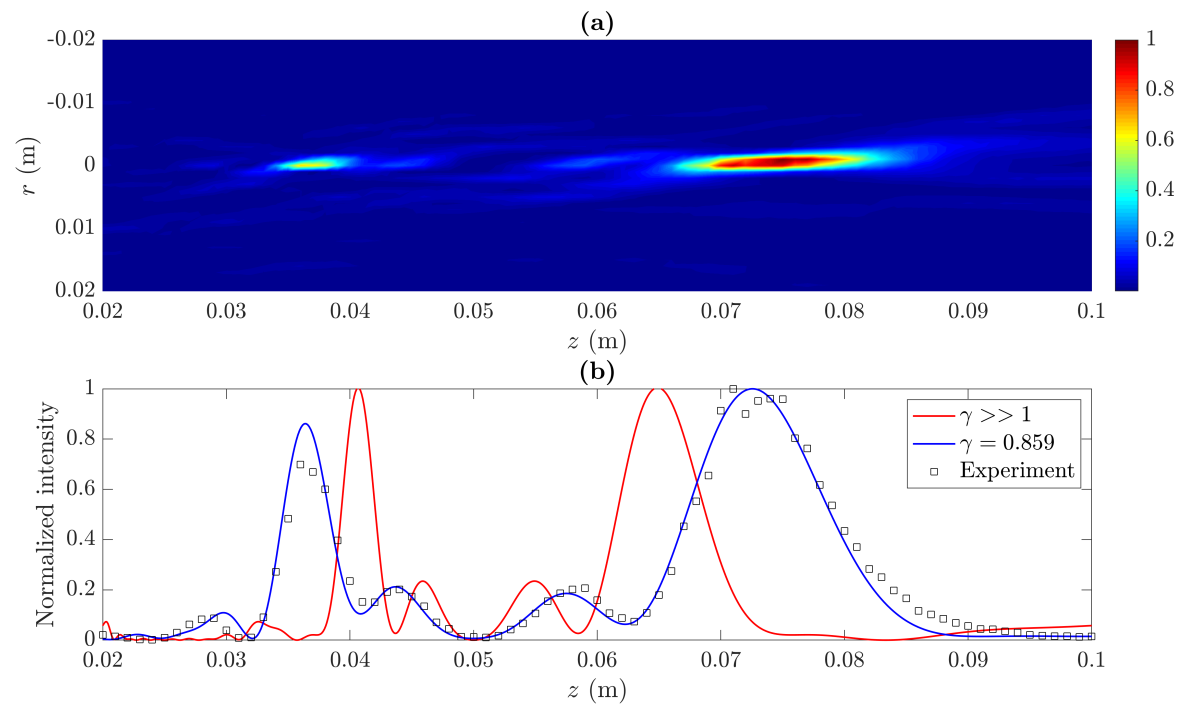

Figure 8.8: Experimental results: (a) Measured intensity map ;(b) Measured (black squares) and simulated (blue line) focusing profiles for the MbZP built lens compared to the $\gamma>>1$ case (red line).

As can be observed, the manufactured lens with $\gamma=0.859$ provides focal distances of $z_{1}=36.4 \mathrm{~mm}$ and $z_{2}=72.6 \mathrm{~mm}$, which differ from those corresponding to the $\gamma>>1$ case $\left(z_{1}=40.6 \mathrm{~mm}\right.$ and $\left.z_{2}=64.9 \mathrm{~mm}\right)$. Therefore, the experimental results demonstrate the focal shift predicted in the theoretical analysis and shown in Figures 8.4 and 8.5. However, there is also a noticeable distortion in the focusing profile and both foci are not even anymore. Thus, there is a trade off between the focal shifting range and the maximum tolerable distortion in the MbZP focusing profile.

Figure 8.9 shows the effect that the adjustment of the operating frequency has on the performance of the MbZP lens. Figure 8.9(a) depicts the location of both foci against the operating frequency for the built MbZP shown in Figure 8.7(a). This MbZP lens has been designed to operate at a design frequency of $f=1 \mathrm{MHz}$ and the results shown in Figure 8.9(a) have been numerically computed 

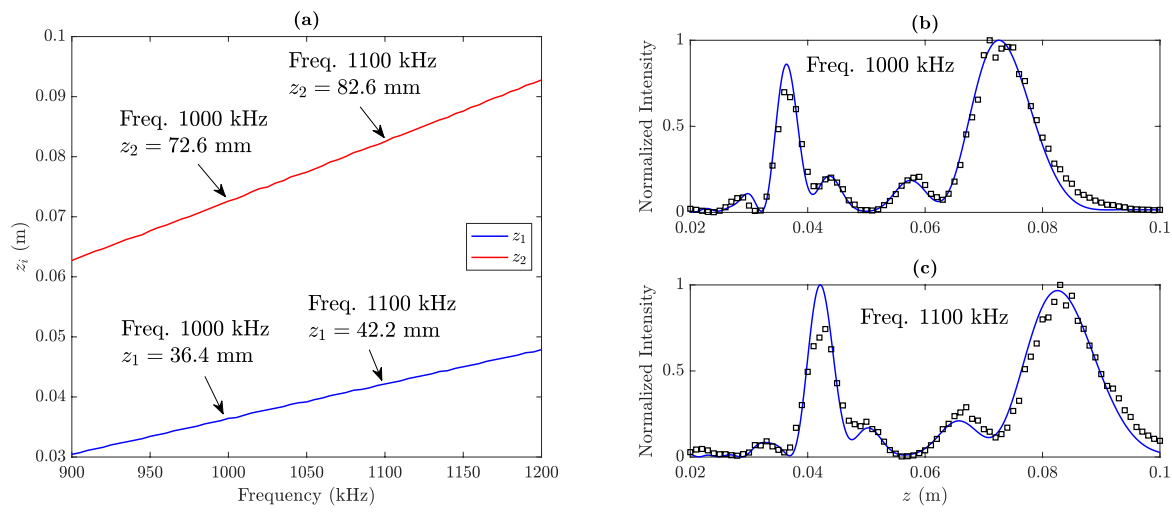

Figure 8.9: (a) Numerically computed focal distances as a function of the working frequency; experimental (black squares) and simulated (blue line) focusing profiles at: (b) $1 \mathrm{MHz}$ and (c) $1.1 \mathrm{MHz}$.

using equation 8.6. As can be observed from the figure, both foci shift linearly with the operating frequency, although at different rates. Thus, the separation between both foci augments with the operating frequency. Figures 8.9(b) and 8.9(c) show the experimental MbZP focusing profiles measured for comparison purposes at $f=1 \mathrm{MHz}$ (design frequency) and $f=1.1 \mathrm{MHz}$, respectively. The separations between both foci are around the numerically computed values, which correspond to $36.2 \mathrm{~mm}$ in the $f=1 \mathrm{MHz}$ case and $40.4 \mathrm{~mm}$ in the $1.1 \mathrm{MHz}$ case. Therefore, Figures 8.9 (b) and 8.9 (c) experimentally demonstrate the effect that the operating frequency has on the MbZP focusing profile.

\subsection{Conclusion}

In this work, we present a method for designing bifocal ZPs using M-bonacci binary sequences in the ultrasound domain. The distance between both foci is related with the $\mathrm{M}$-bonacci sequence ratio. In addition, a new $\gamma$ design parameter is presented, which can be used to finely adjust the foci separation, at the expense of increasing the focusing profile distortion. Experimental results agree with numerical simulations, demonstrating the viability of these type of lenses in high wavelength domains, such as microwaves or ultrasound transmission. 


\section{References}

[1] J. Chen, J. Xiao, D. Lisevych, A. Shakouri, and Z. Fan, "Deep-subwavelength control of acoustic waves in an ultra-compact metasurface lens," Nature Communications, vol. 9, p. 4920, dec 2018.

[2] Y. Li, B. Liang, X. Tao, X.-f. Zhu, X.-y. Zou, and J.-c. Cheng, "Acoustic focusing by coiling up space," Applied Physics Letters, vol. 101, p. 233508, dec 2012.

[3] M. Molerón, M. Serra-Garcia, and C. Daraio, "Acoustic Fresnel lenses with extraordinary transmission," Applied Physics Letters, vol. 105, p. 114109, sep 2014.

[4] Y. Li, G. Yu, B. Liang, X. Zou, G. Li, S. Cheng, and J. Cheng, "Threedimensional Ultrathin Planar Lenses by Acoustic Metamaterials," Scientific Reports, vol. 4, p. 6830, may 2015.

[5] J. Lan, Y. Li, Y. Xu, and X. Liu, "Manipulation of acoustic wavefront by gradient metasurface based on Helmholtz Resonators," Scientific Reports, vol. 7, p. 10587, dec 2017.

[6] S. Jiménez-Gambín, N. Jiménez, J. M. Benlloch, and F. Camarena, "Holograms to Focus Arbitrary Ultrasonic Fields through the Skull," Physical Review Applied, vol. 12, p. 014016, jul 2019.

[7] S. Pérez-López, J. M. Fuster, I. V. Minin, O. V. Minin, and P. Candelas, "Tunable subwavelength ultrasound focusing in mesoscale spherical lenses using liquid mixtures," Scientific Reports, vol. 9, p. 13363, dec 2019.

[8] D. Veira Canle, T. Kekkonen, J. Mäkinen, T. Puranen, H. J. Nieminen, A. Kuronen, S. Franssila, T. Kotiaho, A. Salmi, and E. Hæggström, "Practical realization of a sub- $\lambda / 2$ acoustic jet," Scientific Reports, vol. 9, p. 5189, dec 2019 .

[9] D. C. Calvo, A. L. Thangawng, M. Nicholas, and C. N. Layman, "Thin Fresnel zone plate lenses for focusing underwater sound," Applied Physics Letters, vol. 107, p. 014103, jul 2015.

[10] S. Pérez-López, J. M. Fuster, P. Candelas, C. Rubio, and F. Belmar, "On the use of phase correction rings on Fresnel zone plates with ultrasound piston emitters," Applied Physics Letters, vol. 112, p. 264102, jun 2018.

[11] D. Tarrazó-Serrano, S. Pérez-López, P. Candelas, A. Uris, and C. Rubio, "Acoustic Focusing Enhancement In Fresnel Zone Plate Lenses," Scientific Reports, vol. 9, p. 7067, dec 2019. 
[12] R. S. Rodrigues Ribeiro, P. Dahal, A. Guerreiro, P. A. S. Jorge, and J. Viegas, "Fabrication of Fresnel plates on optical fibres by FIB milling for optical trapping, manipulation and detection of single cells," Scientific Reports, vol. 7, p. 4485, dec 2017.

[13] H. D. Hristov and J. M. Rodriguez, "Design Equation for Multidielectric Fresnel Zone Plate Lens," IEEE Microwave and Wireless Components Letters, vol. 22, pp. 574-576, nov 2012.

[14] G. Clement, H. Nomura, and T. Kamakura, "Ultrasound field measurement using a binary lens," IEEE Transactions on Ultrasonics, Ferroelectrics, and Frequency Control, vol. 62, pp. 350-359, feb 2015.

[15] S. Pérez-López, J. M. Fuster, P. Candelas, and C. Rubio, "On the focusing enhancement of Soret zone plates with ultrasound directional transducers," Applied Physics Letters, vol. 114, p. 224101, jun 2019.

[16] J. A. Monsoriu, A. Calatayud, L. Remon, W. D. Furlan, G. Saavedra, and P. Andres, "Bifocal Fibonacci Diffractive Lenses," IEEE Photonics Journal, vol. 5, pp. 3400106-3400106, jun 2013.

[17] F. Machado, V. Ferrando, W. D. Furlan, and J. A. Monsoriu, "Diffractive m-bonacci lenses," Optics Express, vol. 25, p. 8267, apr 2017.

[18] G. Saavedra, W. D. Furlan, and J. A. Monsoriu, "Fractal zone plates," Optics Letters, vol. 28, p. 971, jun 2003.

[19] W. D. Furlan, G. Saavedra, and J. A. Monsoriu, "White-light imaging with fractal zone plates," Optics Letters, vol. 32, p. 2109, aug 2007.

[20] O. Mendoza-Yero, M. Fernández-Alonso, G. Mínguez-Vega, J. Lancis, V. Climent, and J. A. Monsoriu, "Fractal generalized zone plates," Journal of the Optical Society of America A, vol. 26, p. 1161, may 2009.

[21] V. Ferrando, F. Giménez, W. D. Furlan, and J. A. Monsoriu, "Bifractal focusing and imaging properties of Thue-Morse Zone Plates," Optics Express, vol. 23, p. 19846, jul 2015.

[22] T. Xia, S. Cheng, and S. Tao, "Generation of three equal-intensity foci based on a modified composite zone plate," Optik, vol. 159, pp. 150-156, apr 2018.

[23] J. Fuster, S. Pérez-López, P. Candelas, and C. Rubio, "Design of BinarySequence Zone Plates in High Wavelength Domains," Sensors, vol. 18, p. 2604, aug 2018. 
[24] L. Nie, X. Cai, K. Maslov, A. Garcia-Uribe, M. A. Anastasio, and L. V. Wang, "Photoacoustic tomography through a whole adult human skull with a photon recycler," Journal of Biomedical Optics, vol. 17, p. 110506, nov 2012.

[25] M. Chen, H. J. Knox, Y. Tang, W. Liu, L. Nie, J. Chan, and J. Yao, "Simultaneous photoacoustic imaging of intravascular and tissue oxygenation," Optics Letters, vol. 44, p. 3773, aug 2019.

[26] G. ter Haar and C. Coussios, "High intensity focused ultrasound: Physical principles and devices," International Journal of Hyperthermia, vol. 23, pp. 89-104, jan 2007.

[27] D. Suo, Z. Jin, X. Jiang, P. A. Dayton, and Y. Jing, "Microbubble mediated dual-frequency high intensity focused ultrasound thrombolysis: An In vitro study," Applied Physics Letters, vol. 110, p. 023703, jan 2017.

[28] S. GyP, L. DY, and H. G, "What is on the Horizon for Hyperthermic Cancer Therapy?," Journal of Traditional Medicine \& Clinical Naturopathy, vol. 06, no. 02, 2017.

[29] J. C. Simon, O. A. Sapozhnikov, V. A. Khokhlova, Y.-N. Wang, L. A. Crum, and M. R. Bailey, "Ultrasonic atomization of tissue and its role in tissue fractionation by high intensity focused ultrasound," Physics in Medicine and Biology, vol. 57, pp. 8061-8078, dec 2012.

[30] J. S. Jeong, J. M. Cannata, and K. K. Shung, "Dual-Focus Therapeutic Ultrasound Transducer for Production of Broad Tissue Lesions," Ultrasound in Medicine \& Biology, vol. 36, pp. 1836-1848, nov 2010.

[31] Jong Seob Jeong, "Dual concentric-sectored HIFU transducer with phaseshifted ultrasound excitation for expanded necrotic region: a simulation study," IEEE Transactions on Ultrasonics, Ferroelectrics, and Frequency Control, vol. 60, pp. 924-931, may 2013. 


\title{
Chapter 9
}

\section{Liquid-liquid Core-shell Configurable Mesoscale Spherical Acoustic Lens with Subwavelength Focusing}

\author{
S. Pérez-López, P. Candelas, J. M. Fuster, C. Rubio, O. V. Minin, \\ and I. V. Minin, "Liquid-liquid core-shell configurable mesoscale \\ spherical acoustic lens with subwavelength focusing," Applied \\ Physics Express, vol. 12, no. 8, p. 087001, Aug. 2019. ( 2019 \\ The Japan Society of Applied Physics. \\ https://doi.org/10.7567/1882-0786/ab2c7a
}

\section{Abstract}

In this work, we present a lens based on a thin hollow ABS spherical container structure, which can be filled up with different compatible liquids. The acoustic jet can be dynamically shaped by either shifting the operating frequency or modifying the geometry of the lens. We show for the first time that a spherical Ethanol ABS core-shell acoustical lens immersed in water with low diameter-to-wavelength ratio equal to 6.67 and refractive index of 1.24 achieves a focusing spot narrower than $0.85 \lambda$. Experimental measurements validate simulation results and demonstrate 
the viability of these configurable spherical lenses in underwater acoustic focusing applications.

\subsection{Introduction}

Wave focusing is a hot topic in several areas of physics, including optics [1, 2], microwaves [3, 4] and acoustics [5-7]. In this sense, spherical lenses have attracted close attention since the invention of the optical microscope [8]. In acoustics, spherical refractive gas-filled [9] and liquid-filled [10] lenses with high diameterto-wavelength ratio $(D / \lambda)$, where the geometrical optics approach is valid, were previously investigated. A liquid-filled acoustic lens with an acoustically transparent spherical shell made of $\mathrm{ABS}$ plastic with a relative diameter of $D / \lambda=17$ at $100 \mathrm{kHz}$ was studied [11]. A liquid-filled spherical acoustic lens with the focal point inside the lens [12] was also investigated. Nevertheless, subwavelength acoustic focusing was never experimentally demonstrated in these works.

Some alternative methods to achieve subwavelength focusing $[13,14]$ include non-linear harmonic generation [15] or time-reversal mirrors [16, 17]. However, these alternative methods are low-efficiency processes due to different drawbacks such as ultrasound absorption or low energy translation between frequencies, and thus, spherical lenses are more appealing. Theoretically, based on an analogy to optical photonic nanojet research [18-23], it was recently shown $[24,25]$ that acoustic focusing can be achieved using mesoscale solid or/and metamaterial lenses that produce jet-like focus, known as acustojets, when the size of the lens is less than $10 \lambda$ and the geometrical optics approach is not valid. Experimentally, it has been shown that it is possible to obtain a diffraction limited focal spot with mesoscale solid spherical [26] and cylindrical [27] acoustic lenses, which can enhance the capabilities of ultrasound imaging in several areas such as biomedical applications or acoustic microscopy. Acoustic jets are scattered non-evanescent beams of acoustic waves that propagate a short distance through the shadow region of the spherical/cylindrical lens, with subwavelength or even beyond the diffraction limit transversal resolution. The physics behind the jet generation can be described using Mie scattering theory $[9,24,26]$.

It should be noted that there is a fundamental difference in the focusing properties between cylindrical (2D) and spherical (3D) lenses and it is not correct to generalize the results of a $2 \mathrm{D}$ lens to a $3 \mathrm{D}$ lens directly [28].

Moreover, solid materials with good acoustic properties are extremely rare, and liquid lenses have an advantage over solid lenses because of the lack of transverse sound velocity (shear waves). However, until now, experimental realization 
of liquid core-shell mesoscale spherical acoustic lenses with resolution beyond the wavelength has not been achieved. In this work, we propose a configurable liquid core-shell mesoscale acoustic spherical lens which can modify its acoustic jet-like subwavelength focusing profile.

\subsection{Results}

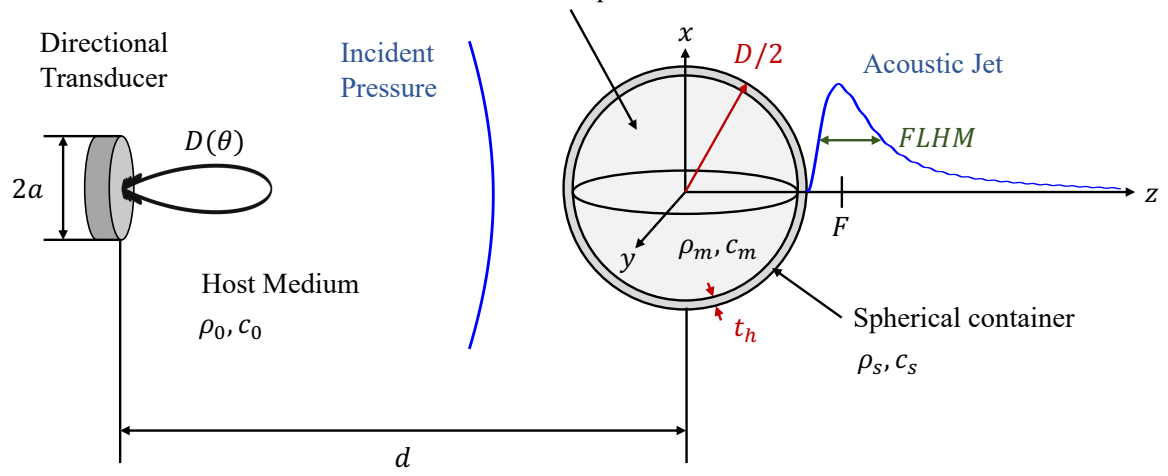

Figure 9.1: Schematic of the configurable spherical lens. [( ${ }^{\circledR} 2019$ The Japan Society of Applied Physics]

Figure 9.1 depicts a hollow spherical lens of thickness $t_{h}<<\lambda$, where $\lambda$ is the wavelength in the host medium, and diameter $D$ filled with an inner liquid characterized by its density $\rho_{m}$ and sound speed $c_{m}$. The spherical lens is placed at a distance $d$ from a directional transducer [29] and immersed in a host medium with density $\rho_{0}$ and sound speed $c_{0}$. The refractive index of the inner liquid is then defined as $n=c_{0} / c_{m}$, and provides helpful information about the sound speed contrast between both media. The acoustic jet focusing profile on the near-field region can be shaped by modifying the inner liquid inside the spherical lens, and can be characterized by the focal distance $(F)$, the focal intensity $\left(I_{F}\right)$, the full length half maximum (FLHM) and the full width half maximum (FWHM). These parameters provide information about the jet capability to focus the incident energy into a certain location.

The acoustic pressure generated by the lens has been calculated using a Finite Element Method (FEM) model implemented in the commercial software COMSOL Multiphysics. In all simulations, the host medium is water and the spherical lens container material is the thermoplastic polymer ABS. The hollow 
spherical container has a thickness $t_{h}=0.25 \mathrm{~mm}$ and an outer diameter $D=40$ $\mathrm{mm}$. In all simulations, the transducer is modelled as a constant pressure condition of $p_{0}=1 \mathrm{~Pa}$ placed at a distance $d=350 \mathrm{~mm}$ from the center of the lens.

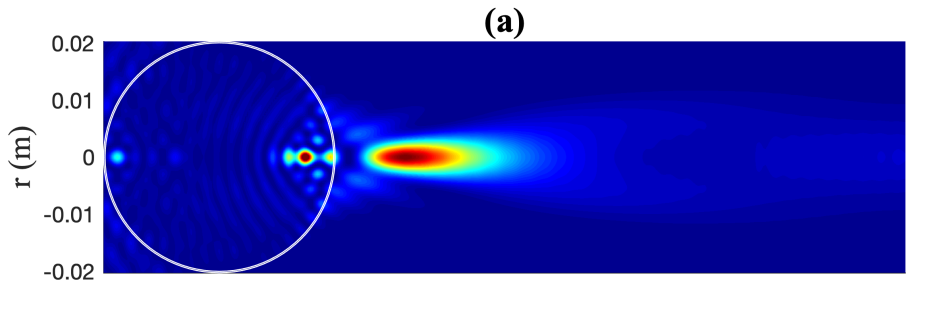

(b)

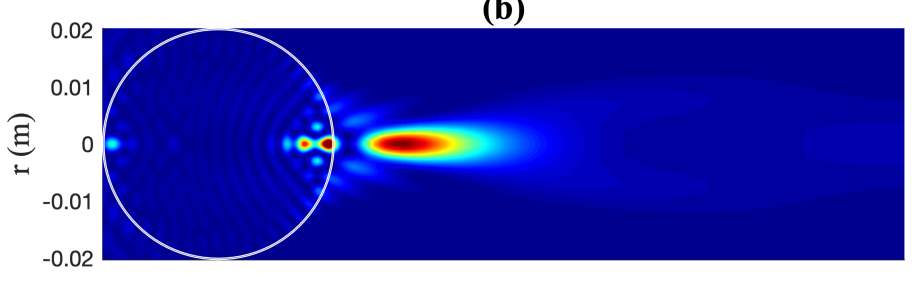

(c)

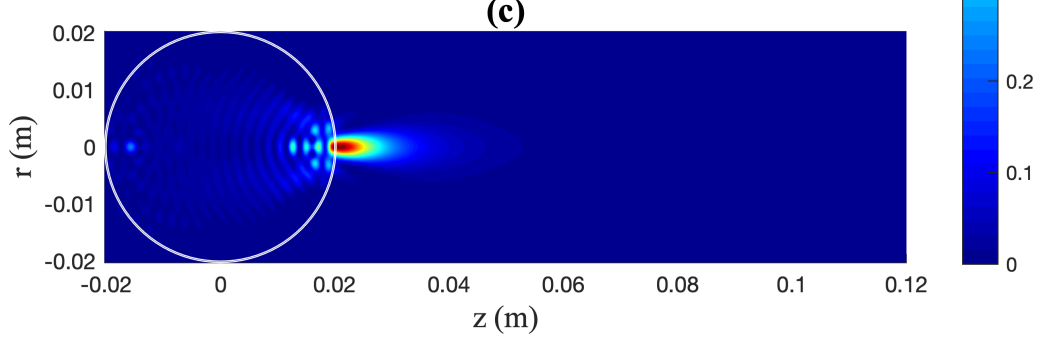

Figure 9.2: Normalized intensity maps for different materials: (a) Hexane, (b) Ethanol and (c) Methyl lodide. [`2019 The Japan Society of Applied Physics]

In analogy to optical photonic nanojet research, the surrounding medium should have a lower sound speed than the inner liquid of the lens [24]. Figure 9.2 shows the numerically computed intensity maps for different inner liquids. Their acoustic properties, as well as those of the host medium and the container material, are shown in Table 9.1 for a temperature of $T=20^{\circ} \mathrm{C}$. In all three cases the operating frequency is set to $f=250 \mathrm{kHz}$, which corresponds to $\lambda=6$ $\mathrm{mm}$. In this case, $D=6.67 \lambda$ and $t_{h}=0.042 \lambda$. As can be observed from Figure 9.2 , the acoustic properties of the inner material have a significant impact on the acoustic jet focusing profile. Figures 9.2(a), 9.2(b) and 9.2(c) show the acoustic 
jet generated by a hollow spherical lens when the inner liquid is Hexane, Ethanol and Methyl lodide, respectively. Table 9.2 shows the values of all four acoustic jet parameters depicted in Figure 9.2. Hexane (Figure 9.2(a)) provides the most extended focal area with the lowest focal intensity. Moreover, it corresponds to the widest focal length of $32.5 \mathrm{~mm}$. Ethanol (Figure $9.2(\mathrm{~b})$ ) has a very low refractive index, close to Hexane, and thus it provides a similar focal length of $32 \mathrm{~mm}$. Methyl lodide (Figure 9.2(c)) has a higher refractive index than the other two materials, which results in a smaller focal length of $21.05 \mathrm{~mm}$. This material also provides the highest focal intensity and the narrowest lateral resolution $(0.472 \lambda)$ beyond the diffraction limit [19]. To further increase the resolution of this jet, the diameter of the sphere could be augmented. However, Methyl lodide is a very toxic material and the experimental measurements have been carried out using Ethanol because of health concerns.

\begin{tabular}{lrcc}
\hline Material & $\rho\left(\mathrm{kg} / \mathrm{m}^{3}\right)$ & $c(\mathrm{~m} / \mathrm{s})$ & $n$ \\
\hline Water & 1000 & 1500 & 1.00 \\
ABS & 1050 & 2250 & 0.67 \\
Hexane & 655 & 1203 & 1.25 \\
Ethanol & 789 & 1207 & 1.24 \\
Merthyl lodide & 2280 & 978 & 1.53 \\
\hline
\end{tabular}

Table 9.1: Material acoustic properties.

It is worth noting that despite Hexane having a slightly higher refractive index than Ethanol, it achieves a longer focal distance compared to Ethanol. In optics, a higher refractive index usually results in a shorter focal distance and a higher focal intensity $[18,19]$. However, mechanical waves behave differently than electromagnetic waves, and as shown in Table 9.1, there are several parameters that influence the focusing properties of the acoustic jet. The difference between the refractive indexes of both Ethanol and Hexane is very low, and both lenses are expected to achieve similar focal distances. Nevertheless, Ethanol has a higher density compared to Hexane, which makes its acoustic impedance higher too. This difference in impedance is the real cause behind the shorter focal distance and the lower intensity level of the Hexane lens compared to the Ethanol lens. Therefore, the density of the inner liquid must also be considered, as it modifies the acoustic impedance of the lens resulting in variations on both focal distance and intensity level.

Once the inner liquid has been selected, an interesting parameter which could be used to achieve a finer adjustment onto the focusing profile is the operating frequency. Figure 9.3 shows the variation of all acoustic jet parameters against the operating frequency when the spherical lens is filled with Ethanol. In the simulations, the incident pressure at the lens is not constant as a directional 
Chapter 9. Liquid-liquid Core-shell Configurable Mesoscale Spherical Acoustic Lens with Subwavelength Focusing

\begin{tabular}{lcccc}
\hline Material & $F(\lambda)$ & $I_{F}$ & FLHM $(\lambda)$ & FWHM $(\lambda)$ \\
\hline Hexane & 5.42 & 0.248 & 3.085 & 0.757 \\
Ethanol & 5.33 & 0.339 & 2.893 & 0.715 \\
Methyl lodide & 3.51 & 1.000 & - & 0.472 \\
\hline
\end{tabular}

Table 9.2: Acoustic jet parameters of the different materials shown in Figure 9.2 $(f=250 \mathrm{kHz})$.

(a)

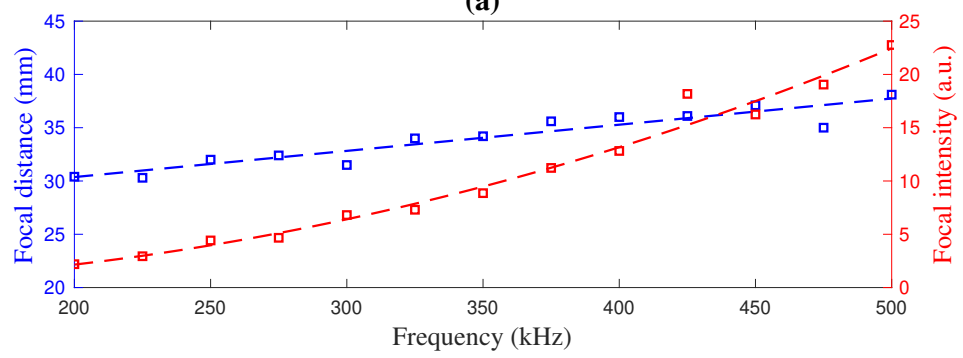

(b)

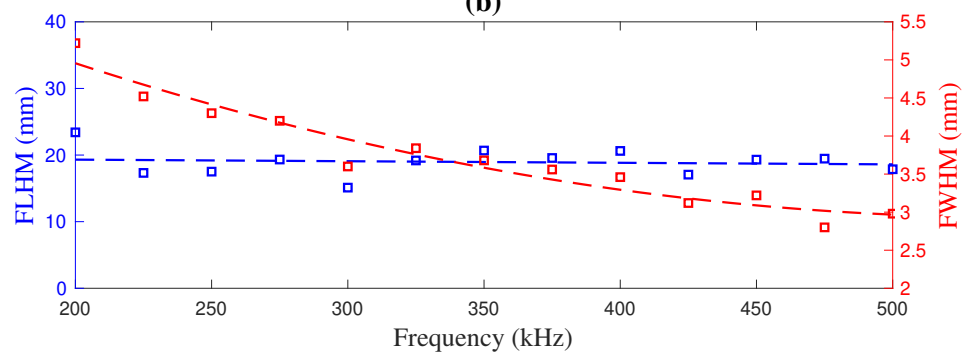

Figure 9.3: Acoustic jet parameters as a function of the operating frequency. (a) Focal distance (blue) and focal intensity (red), (b) FLHM (blue) and FWHM (red). [` 2019 The Japan Society of Applied Physics]

transducer is being considered. The transducer becomes more directional when the operating frequency is increased, due to its bigger size in terms of the wavelength. As can be observed from Figure 9.3, the operating frequency induces a linear shift on the focal distance and has almost no effect over the longitudinal resolution, which is determined by the FLHM parameter. The focal intensity increases quadratically with the operating frequency, while the FWHM parameter decreases also with a quadratic tendency, which translates into a higher lateral resolution. Thus, the operating frequency could be used as a dynamic control parameter to linearly shift the focal length. 

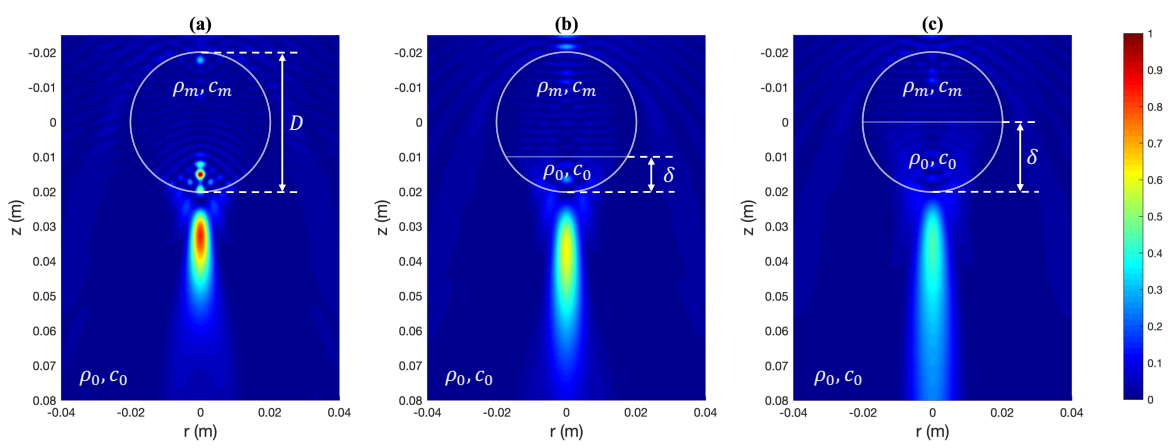

Figure 9.4: Simulated intensity maps for different $\delta$ values: (a) $\delta=0$, (b) $\delta=D / 4$ and (c) $\delta=D / 2$. All values are normalized to the maximum intensity, which in this case is achieved when $\delta=0$. The frequency is $f=250 \mathrm{kHz}$. [ ${ }^{\odot} 2019$ The Japan Society of Applied Physics]

In order to provide additional control on the acoustic jet focusing profile, the effect of the lens geometry has also been considered [30-32]. When the lens inner liquid is immiscible with the host medium, the geometry of the acoustic lens can be dynamically modified by introducing a certain amount of the host medium liquid inside the lens. Neglecting the thickness of the sphere, the resulting structure behaves as a truncated spherical lens, where the size of the truncated section depends on the amount of host medium introduced inside the lens. The variation on the lens geometry can be implemented by attaching an input and output connection to the lens container. The input connection allows the introduction of the desired amount of host medium liquid, while the exceeding inner liquid is evacuated through the output connection. This mechanism provides a dynamical and fast procedure to modify the focusing profile of the acoustic jet.

Figure 9.4 shows simulation results for three configurations in which different amounts of host medium liquid have been introduced inside the lens. The host medium is water and the inner liquid is Hexane, an hydrocarbon immiscible with water. As water is denser than Hexane, it occupies the bottom of the spherical lens, while Hexane is isolated on top of the water layer. The directional transducer is located in this case above the lens pointing downwards and the acoustic jet is oriented vertically as shown in Figure 9.4. A design parameter, $\delta$, depicted in Figures 9.4(b) and 9.4(c), has been defined as the axial length corresponding to the volume of host medium liquid inside the lens. As can be observed from Figure 9.4, when the spherical lens is truncated, the focal area is outspread, decreasing the focal intensity while both the FWHM and the FLHM parameters increase their values. 
(a)

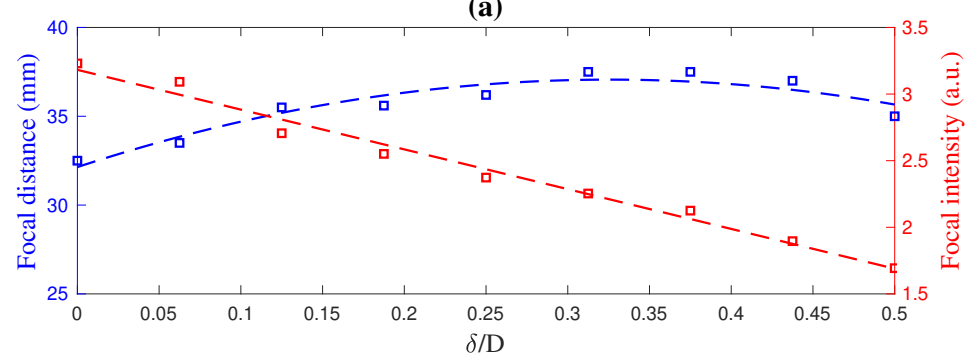

(b)

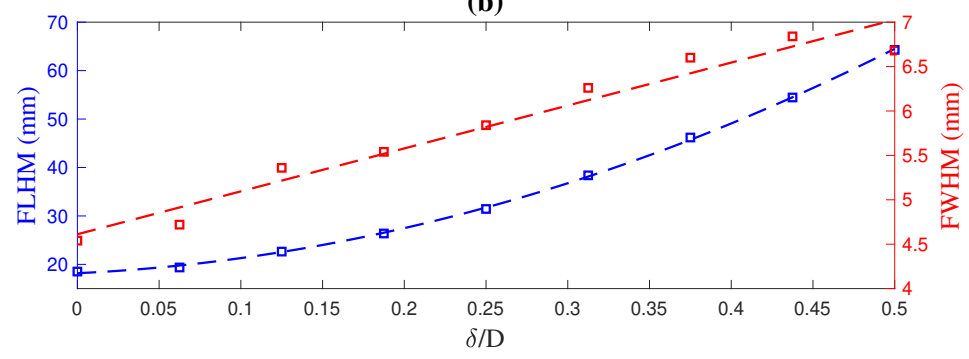

Figure 9.5: Acoustic jet parameters as a function of $\delta$. (a) Focal distance (blue) and focal intensity (red), (b) FLHM (blue) and FWHM (red). $f=250 \mathrm{kHz}$. [` 2019 The Japan Society of Applied Physics]

Figure 9.5 shows the variation of all acoustic jet parameters against $\delta$ when the inner liquid is Hexane and the host medium is water. The focal length varies quadratically with $\delta$ with a local maximum around $\delta=0.3$. Besides, when the $\delta$ parameter augments its value, that is when the truncated size becomes larger, both FLHM and FWHM parameters increase while the focal intensity diminishes almost linearly. Therefore, the variation of the lens geometry can be used to dynamically soften and spread out the focal area of the acoustic jet.

An alternative control parameter would be the volume fraction, $V_{F}$, that is the ratio between the host medium volume inside the lens and the total liquid volume (host medium and inner liquid). The $V_{F}$ parameter can be easily computed and is related to the $\delta / D$ parameter through the following expression

$$
V_{F}=\frac{V_{h m}}{V_{\text {total }}}=3\left(\frac{\delta}{D}\right)^{2}-2\left(\frac{\delta}{D}\right)^{3},
$$

with $V_{\text {total }}=V_{i l}+V_{h m}$, being $V_{i l}$ the inner liquid volume and $V_{h m}$ the host medium volume inside the lens. 


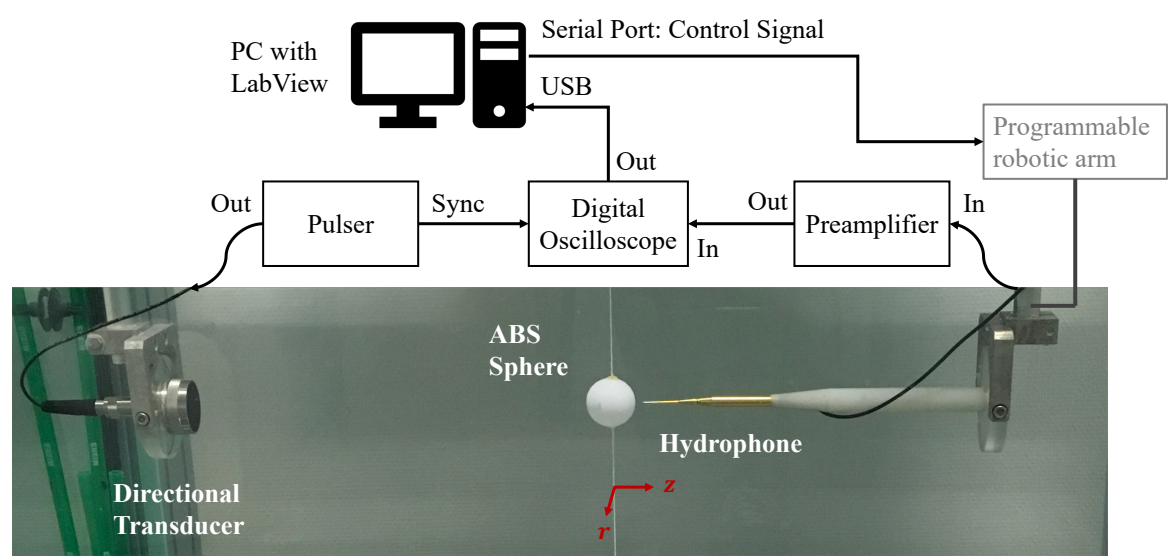

Figure 9.6: Experimental Set-up. [`2019 The Japan Society of Applied Physics]

Experimental measurements have been carried out in order to validate the configurable spherical lens. Figure 9.6 shows the experimental set-up, where an underwater 3D automated positioning system with a spatial resolution of $1 \times 1 \times 1$ $\mathrm{mm}^{3}$ is used inside a water tank. An Imasonic $250 \mathrm{kHz}$ piston transducer with a $30 \mathrm{~mm}$ active diameter is used as emitter. A needle hydrophone from Precision Acoustics Ltd., with a diameter of $1.5 \mathrm{~mm}$, is used as receiver. The transmitted signal is generated using a Panametrics 5077PR Pulser, whereas the received signal is acquired and sampled using a digital oscilloscope from Pico Technology. The measurement process is automated using a LabView program, which controls both the robotic arm and the digital oscilloscope.

Figures 9.7(a) and 9.7(b) show simulated and measured intensity maps, respectively, for a frequency of $f=250 \mathrm{kHz}$. Figures 9.8(a) and 9.8(b) depict the longitudinal and radial cuts of the intensity maps shown in Figure 9.7. Simulations are depicted in red, while the blue lines correspond to experimental measurements. As can be observed from Figures 9.7 and 9.8, simulations and experimental measurements agree very well, validating the use of these configurable spherical lenses for underwater ultrasound focusing. As can be observed from Figure 9.8(a), the measured focal distance $(F=32.00 \mathrm{~mm})$ is identical to that simulated with the FEM software. However, the focal area spreads out slightly in the experimental measurements in comparison to the simulation results. Thus, the measured FLHM and FWHM are $3.330 \lambda$ and $0.847 \lambda$, respectively, which result in an increase of $13.07 \%$ and $18.69 \%$ compared to their simulated counterparts. 


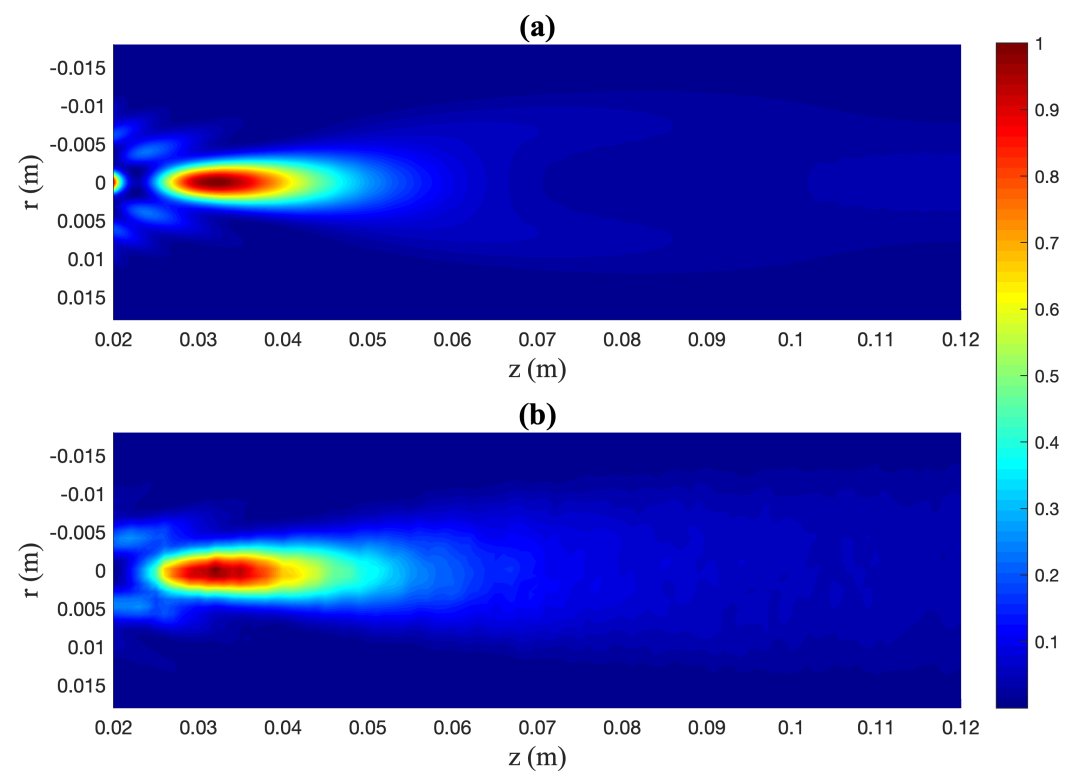

Figure 9.7: Normalized intensity maps: (a) simulation results and (b) experimental measurements. [ ${ }^{\circledR} 2019$ The Japan Society of Applied Physics]

\subsection{Discussion}

In this paper, a configurable liquid-liquid core-shell mesoscale spherical acoustic lens with a diameter of $6.67 \lambda$ and a refractive index of 1.24 capable of providing subwavelength resolution has been proposed. The choice of the inner liquid allows a coarse control of the acoustic jet properties. Once the inner liquid is selected, finer tunability can be achieved by shifting the operating frequency, which has a significant effect over the focal intensity and the FWHM parameter. Moreover, if the inner liquid is immiscible with the host medium, additional control on the jet focusing profile can be provided by introducing a certain amount of the host medium liquid inside the hollow sphere and thus, modifying the geometry of the lens. An Ethanol-filled lens with an ABS shell has been experimentally characterized, showing good agreement with numerical simulations and providing a simple method to achieve subwavelength focusing $(0.847 \lambda)$. It has also been shown that the use of an inner liquid with a lower sound speed leads to an increase in lateral resolution beyond the diffraction limit. Thus, this work opens new possibilities to implement and design compact focusing/imaging systems with high resolution and controllable focal distances, that can be appealing to numerous researchers 
(a)
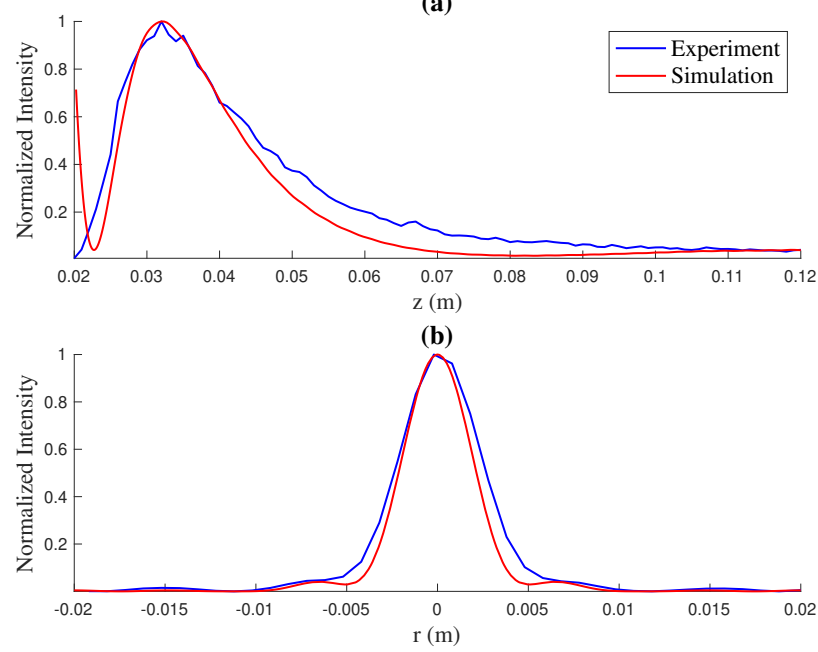

Figure 9.8: (a) Longitudinal cut $(r=0)$ and (b) transversal cut $(z=F)$. Experimental measurements (blue lines) and simulation results (red lines). [( 2019 The Japan Society of Applied Physics]

working in acoustics.

\section{References}

[1] Q. Meng, X. Zhang, L. Cheng, P. Cao, Y. Li, H. Zhang, and G. Wang, "Deep subwavelength focusing of light by a trumpet hyperlens," Journal of Optics, vol. 13, p. 075102, jul 2011.

[2] M. Papaioannou, E. Plum, E. T. Rogers, and N. I. Zheludev, "All-optical dynamic focusing of light via coherent absorption in a plasmonic metasurface," Light: Science \& Applications, vol. 7, pp. 17157-17157, mar 2018.

[3] H. D. Hristov and J. M. Rodriguez, "Design Equation for Multidielectric Fresnel Zone Plate Lens," IEEE Microwave and Wireless Components Letters, vol. 22, pp. 574-576, nov 2012.

[4] T. Ding, J. Yi, H. Li, H. Zhang, and S. N. Burokur, "3D field-shaping lens using all-dielectric gradient refractive index materials," Scientific Reports, vol. 7, p. 782, dec 2017. 
[5] N. Jiménez, V. Romero-García, R. Picó, L. M. Garcia-Raffi, and K. Staliunas, "Nonlinear focusing of ultrasonic waves by an axisymmetric diffraction grating embedded in water," Applied Physics Letters, vol. 107, p. 204103, nov 2015.

[6] Y. Ge, H.-x. Sun, C. Liu, J. Qian, S.-q. Yuan, J.-p. Xia, Y.-j. Guan, and S.-y. Zhang, "Acoustic focusing by an array of heat sources in air," Applied Physics Express, vol. 9, p. 066701, jun 2016.

[7] J.-p. Xia, H.-x. Sun, Q. Cheng, Z. Xu, H. Chen, S.-q. Yuan, S.-y. Zhang, Y. Ge, and Y.-j. Guan, "Theoretical and experimental verification of acoustic focusing in metal cylinder structure," Applied Physics Express, vol. 9, p. 057301, may 2016.

[8] R. Hooke, Micrographia, or some Physiological Descriptions of Minute Bodies, made by Magnifying Glasses, with Observations and Inquiries thereupon. printed by J. Martyn and J. Allestry, Royal Society, London, 1665.

[9] D. C. Thomas, K. L. Gee, and R. S. Turley, "A balloon lens: Acoustic scattering from a penetrable sphere," American Journal of Physics, vol. 77, pp. 197203, mar 2009.

[10] G. C. Knollman, J. L. S. Bellin, and J. L. Weaver, "Variable-Focus LiquidFilled Hydroacoustic Lens," The Journal of the Acoustical Society of America, vol. 49, pp. 253-261, jan 1971.

[11] E. Belcher, "A multibeam, diver-held sonar using a liquid-filled, spherical acoustic lens," in Proceedings of OCEANS '93, pp. III398-III402, IEEE, 2002.

[12] S. M. Hasheminejad and M. Azarpeyvand, "Sound radiation from a liquidfilled underwater spherical acoustic lens with an internal eccentric baffled spherical piston," Ocean Engineering, vol. 31, pp. 1129-1146, jun 2004.

[13] T. Liu, F. Chen, S. Liang, H. Gao, and J. Zhu, "Subwavelength Sound Focusing and Imaging Via Gradient Metasurface-Enabled Spoof Surface Acoustic Wave Modulation," Physical Review Applied, vol. 11, p. 034061, mar 2019.

[14] F. Ma, J. Chen, and J. H. Wu, "Three-dimensional acoustic sub-diffraction focusing by coiled metamaterials with strong absorption," Journal of Materials Chemistry C, vol. 7, no. 17, pp. 5131-5138, 2019.

[15] D. Rugar, "Resolution beyond the diffraction limit in the acoustic microscope: A nonlinear effect," Journal of Applied Physics, vol. 56, pp. 1338-1346, sep 1984.

[16] J. de Rosny and M. Fink, "Overcoming the Diffraction Limit in Wave Physics Using a Time-Reversal Mirror and a Novel Acoustic Sink," Physical Review Letters, vol. 89, p. 124301, aug 2002. 
[17] A. Sarvazyan, L. Fillinger, and L. R. Gavrilov, "Time-reversal acoustic focusing system as a virtual random phased array," IEEE Transactions on UItrasonics, Ferroelectrics and Frequency Control, vol. 57, pp. 812-817, apr 2010.

[18] A. Heifetz, S.-C. Kong, A. V. Sahakian, A. Taflove, and V. Backman, "Photonic Nanojets," Journal of Computational and Theoretical Nanoscience, vol. 6, pp. 1979-1992, sep 2009.

[19] B. S. Luk'yanchuk, R. Paniagua-Domínguez, I. Minin, O. Minin, and Z. Wang, "Refractive index less than two: photonic nanojets yesterday, today and tomorrow [Invited]," Optical Materials Express, vol. 7, p. 1820, jun 2017.

[20] P. Wu, J. Li, K. Wei, and W. Yue, "Tunable and ultra-elongated photonic nanojet generated by a liquid-immersed core-shell dielectric microsphere," Applied Physics Express, vol. 8, p. 112001, nov 2015.

[21] L. Yue, B. Yan, J. N. Monks, Z. Wang, N. T. Tung, V. D. Lam, O. Minin, and I. Minin, "Production of photonic nanojets by using pupil-masked 3D dielectric cuboid," Journal of Physics D: Applied Physics, vol. 50, p. 175102, may 2017.

[22] L. Yue, B. Yan, J. N. Monks, R. Dhama, Z. Wang, O. V. Minin, and I. V. Minin, "Intensity-Enhanced Apodization Effect on an Axially Illuminated Circular-Column Particle-Lens," Annalen der Physik, vol. 530, p. 1700384, feb 2018.

[23] L. Yue, O. V. Minin, Z. Wang, J. N. Monks, A. S. Shalin, and I. V. Minin, "Photonic hook: a new curved light beam," Optics Letters, vol. 43, p. 771, feb 2018.

[24] O. V. Minin and I. V. Minin, "Acoustojet: acoustic analogue of photonic jet phenomenon based on penetrable 3D particle," Optical and Quantum Electronics, vol. 49, p. 54, feb 2017.

[25] J. H. Lopes, J. P. Leão-Neto, I. V. Minin, O. V. Minin, and G. T. Silva, "A theoretical analysis of jets," in 22nd International Congress on Acoustics, (Buenos Aires, Argentina), 2016.

[26] J. H. Lopes, M. A. B. Andrade, J. P. Leão-Neto, J. C. Adamowski, I. V. Minin, and G. T. Silva, "Focusing Acoustic Beams with a Ball-Shaped Lens beyond the Diffraction Limit," Physical Review Applied, vol. 8, p. 024013, aug 2017.

[27] I. Minin and O. Minin, "Mesoscale Acoustical Cylindrical Superlens," MATEC Web of Conferences, vol. 155, p. 01029, feb 2018. 
[28] Y. E. Geints, A. A. Zemlyanov, O. V. Minin, and I. V. Minin, "Systematic study and comparison of photonic nanojets produced by dielectric microparticles in 2D- and 3D-spatial configurations," Journal of Optics, vol. 20, p. 065606, jun 2018 .

[29] S. Pérez-López, J. M. Fuster, P. Candelas, C. Rubio, and F. Belmar, "On the use of phase correction rings on Fresnel zone plates with ultrasound piston emitters," Applied Physics Letters, vol. 112, p. 264102, jun 2018.

[30] C.-Y. Liu, "Photonic nanojet shaping of dielectric non-spherical microparticles," Physica E: Low-dimensional Systems and Nanostructures, vol. 64, pp. 23-28, nov 2014.

[31] D. Li, X. Wang, J. Ling, and H. Yuan, "Super-narrow focusing and ultralong working distance by different shapes of dielectric microlenses," Optik, vol. 160, pp. 138-145, may 2018.

[32] Y. Zhou, Y. Tang, Y. He, X. Liu, and S. Hu, "Effects of immersion depth on super-resolution properties of index-different microsphere-assisted nanoimaging," Applied Physics Express, vol. 11, p. 032501, mar 2018. 


\title{
Chapter 10
}

\section{Tunable Subwavelength Ultrasound Focusing in Mesoscale Spherical Lenses using Liquid Mixtures}

\author{
S. Pérez-López, J. M. Fuster, I. V. Minin, O. V. Minin, and P. \\ Candelas, "Tunable subwavelength ultrasound focusing in mesoscale \\ spherical lenses using liquid mixtures, " Scientific Reports, vol. 9, \\ no. 1, p. 13363, Sep. 2019. \\ https://doi.org/10.1038/s41598-019-50019-0
}

\section{Abstract}

In this work, we present a configurable spherical lens for underwater focusing applications, which consists on a hollow ABS container filled with a liquid mixture. Two miscible liquids with different sound speeds are required to implement this novel configurable lens. We show that by adjusting the mixing ratio between the volumes of both liquids, the sound speed of the liquid mixture can be accurately selected. This results in a modification of the acoustic jet properties and a continuous tuning on the lens focal length. This procedure can be fully automatized providing a dynamic control mechanism that can shift the lens focal length to 
any desired value inside a continuous range in both directions. Depending on the acoustic properties of the selected liquids, subwavelength resolution or even beyond the diffraction limit resolution can be achieved. We provide experimental measurements for ethanol-water mixtures achieving subwavelength resolution for a certain focal length ranging between 34.6 and $42.8 \mathrm{~mm}$.

\subsection{Introduction}

Photonic nanojets have been a hot topic among the scientific community in the past years due to its high focusing capabilities [1-3]. Nanojets can achieve subwavelength or even beyond the diffraction limit resolution, also known as superfocusing. In acoustics, analogous studies have been presented [4-6] and acoustic jets have been experimentally demonstrated using a solid Rexolite sphere immersed in water [7] and a liquid cylinder shell filled with perfluorinated oil and surrounded by ethanol [8]. Some approaches to achieve acoustic subwavelength focusing include acoustic metasurfaces with coiling-up space structures, Helmholtz resonators or membrane-type structures [9-12]. Other alternatives based on Super Oscillatory Acoustic Lenses (SOAL) [13] or ultra-compact planar metasurfaces have been implemented with subwavelength slits $[14,15]$. However, the manufacturing stage of these kind of devices demands a complete 3D design and optimization of the structure when $3 \mathrm{D}$ focusing is required. In this sense, spherical acoustic lenses are simpler alternatives that can provide 3D superfocusing in the near field for a fixed focal distance.

Having accurate control of the focal length and achieving subwavelength resolution are important requirements in many acoustic focusing applications. In this sense, acoustic systems with dynamic control of the focus are advantageous over devices with a fixed focal length, since they can be adapted to operate in various application scenarios. Nowadays, conventional methods to control the focal length are mainly based on either changing the geometric profile of the lens [16], or modifying the properties of liquid crystals by applying an electric field [17]. On the other hand, the devices capable of providing subwavelength resolution usually have a significant diameter of at least several tens of wavelengths, which results in bulky acoustic systems. With the introduction of acoustic jets, subwavelength focusing is now feasible even with lenses with mesoscale dimensions (diameter to wavelength ratio lower than ten). Mesoscale lenses involve the interaction of acoustic waves with structures of intermediate scale, which are too small to be described by traditional continuum methods. However, there are still no available systems which can provide at the same time both narrow focusing and dynamic control of the focal length while keeping compact dimensions in terms of the wavelength. 
Note that in reference [8] the diameter of the 2D lens was significant and did not meet the mesoscale requirement. In this work, we present a spherical lens with a diameter of $6.7 \lambda$ capable of achieving subwavelength focusing with continuous control of the focal distance and both longitudinal and lateral resolutions.

The spherical lens consists of a hollow container filled with an inner liquid with certain acoustic properties. The sound speed contrast between the inner liquid and the host medium provides the focusing profile of the spherical lens. Thus, the lens focal length can be shifted by replacing the lens inner liquid with another one with different acoustic properties. However, this procedure requires to stop the focusing application during a certain amount of time while the lens is being emptied, cleaned and refilled with the new inner liquid. Alternatively, a more feasible solution would be to have a set of spherical containers with different inner liquids that comprise a certain focal length range and could be faster exchanged. In any case, this procedure would only allow a discrete set of focal lengths and would not be a real configurable lens because it would still require the interruption of the current application in order to modify the focal length.

Most liquid lenses employed for varifocal imaging are able to control their focal distance by modifying their shape [18-20]. Here, we propose a different approach based on filling the spherical lens ABS container with two miscible liquids of different sound speeds. Depending on the mixing ratio between the volumes of both liquids, the sound speed of the liquid mixture can be accurately adjusted to any desired value inside a continuous range, resulting in a shift on the lens focal distance. Moreover, the procedure used to adjust the volume ratio between both liquids can be fully automatized by connecting an input and an output port to the hollow container and determining the type and amount of liquid that has to be introduced to either increase or reduce the sound speed of the liquid mixture, thus achieving an easy-to-implement and dynamic control mechanism to shift the focal distance. Our approach presents two main advantages over other implementations based on spherical lenses. First, it allows a continuous shifting of the lens focal distance and moreover, it can be implemented as a dynamic configurable procedure which does not require the interruption of the focusing application to modify the properties of the acoustic jet. Thus, this method provides an additional degree of flexibility for spherical lenses that has not been previously reported in literature to the best of our knowledge. Experimental measurements for ethanol-water liquid mixtures are presented, showing good agreement with numerical results and demonstrating the viability of this type of lenses. 


\subsection{Results}

An acoustic jet can be characterized by its focal distance $(F)$ and its resolution in both the longitudinal and lateral directions, parameters which specify the location and shape of the jet [6]. In this paper, the focal distance is defined as the distance between the center of the spherical lens and the location of the maximum inside the focus. Longitudinal and lateral resolutions are determined by the full length half maximum (FLHM) and the full width half maximum (FWHM), respectively. Both FLHM and FWHM parameters measure the interval in which the acoustic intensity is reduced to half of its maximum value in their respective axes. All these three parameters depend on the refraction index of the inner liquid, which is defined as $n=c_{0} / c_{m}$, being $c_{0}$ the sound speed of the host medium and $c_{m}$ the sound speed of the inner liquid. As stated above, when two miscible liquids are used, the refraction index of the liquid mixture can be precisely and continuously controlled by varying the mixture volume ratio between both liquids.

The spherical shell of the lens has a thickness $t_{h}<<\lambda$ and a diameter $D$. This container is filled with an inner liquid mixture with density $\rho_{m}$ and sound speed $c_{m}$. The lens is placed inside a water tank with density $\rho_{0}$ and sound speed $c_{0}$ at a distance $d$ from a directional ultrasound transducer. The inner liquid used in this work is a mixture of ethanol and water. The mixture can be characterized by the volume fraction, $\varphi$, defined as

$$
\varphi=\frac{V_{w}}{V_{w}+V_{e}}
$$

where $V_{w}$ and $V_{e}$ are the water an ethanol volumes used to produce the mixture, respectively.

Figure 10.1(a) depicts a schematic concept of the fully automated spherical lens. As stated before, the mixing ratio between both liquids can be controlled by introducing the correct amount of one of the substances through the input connection and extracting the correct volume of the previous mixture through the output connection. Figure 10.1(b) shows the acoustic properties of the ethanolwater liquid mixture as a function of the volume fraction. This measures have been used to obtain second order polynomial fits depicted as dashed lines, which provide continuous characterization of the liquid mixture in the $0<\varphi<0.5$ range. As can be observed from Figure 10.1(b), the sound speed of the liquid mixture is higher than that of both water and ethanol for certain $\varphi$ values, ranging from 1174 to $1547 \mathrm{~m} / \mathrm{s}$ for volume fractions between 0 and $50 \%$.

In this work, the spherical lens has a diameter $D=40 \mathrm{~mm}$ and a shell thickness of $t_{h}=0.25 \mathrm{~mm}$. The thickness of the spherical container is a parameter 
(a)

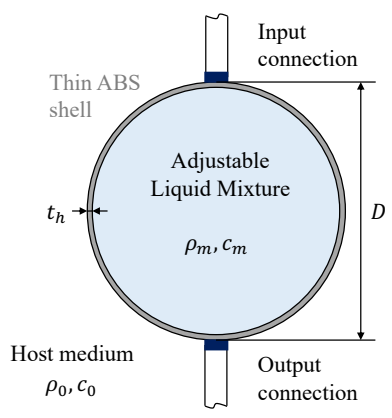

(b)

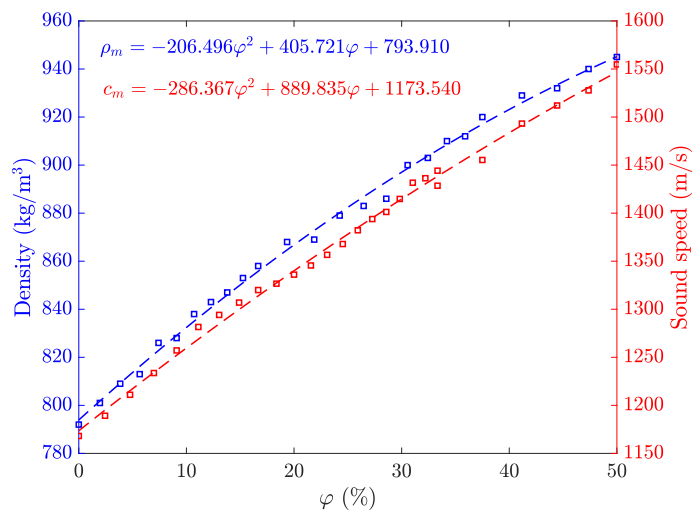

Figure 10.1: (a) Schematic concept of the fully automated lens and (b) acoustic properties (density in blue and sound speed in red) of the liquid mixture against the volume fraction $(\varphi)$. Experiments (squares) and polynomial fits (dashed lines).

that affects the generation of the acoustic jet and can be optimized for a certain inner liquid using FEM simulations. However, when dealing with liquid mixtures, the optimum container thickness is different at distinct volume fractions, and therefore, the whole range of volume fractions should be considered to determine the overall optimum thickness. The working frequency is fixed to $f_{0}=250 \mathrm{kHz}$. Acoustic jets are generated using spherical lenses with diameters between 3 and 30 wavelengths. Under this assumption, the working bandwidth of the proposed lens would be ranging from $110 \mathrm{kHz}$ to $1.12 \mathrm{MHz}$, approximately. This operating bandwidth can be further shrunk due to additional limitations such as the emitter transducer bandwidth. Figure 10.2 shows the acoustic jet parameters as a function of the volume fraction computed using the FEM model described at the Methods section. The properties of the liquid mixture of the FEM model have been obtained from the polynomial fits depicted in Figure 10.1(b). The volume fraction ranges from $0 \%$, which corresponds to pure ethanol with a refraction index of $n_{m}=1.269$, to $20 \%\left(n_{m}=1.1\right)$. In Figure 10.2, squares represent numerical results, whereas dashed lines represent the corresponding polynomial fits. Figure 10.2(a) depicts the focal distance for the spherical lens as a function of the filling fraction. As can be observed, the focal distance follows a smooth quadratic tendency that increases with the $\varphi$ parameter and allows a fine and continuous control of the focus location. Figure 10.2(b) depicts the longitudinal (blue) and lateral (red) resolutions against $\varphi$. As can be observed from the figure, both parameters present a similar quadratic dependence on the $\varphi$ parameter than that of the focal distance. This means that as the volume fraction increases, the resolution of the lens is reduced in both 
the longitudinal and the lateral directions. Moreover, Figure 10.2(b) shows that if subwavelength resolution is required $(\mathrm{FWHM}<5.96 \mathrm{~mm})$, the volume fraction has to be lower than $9 \%$ approximately.
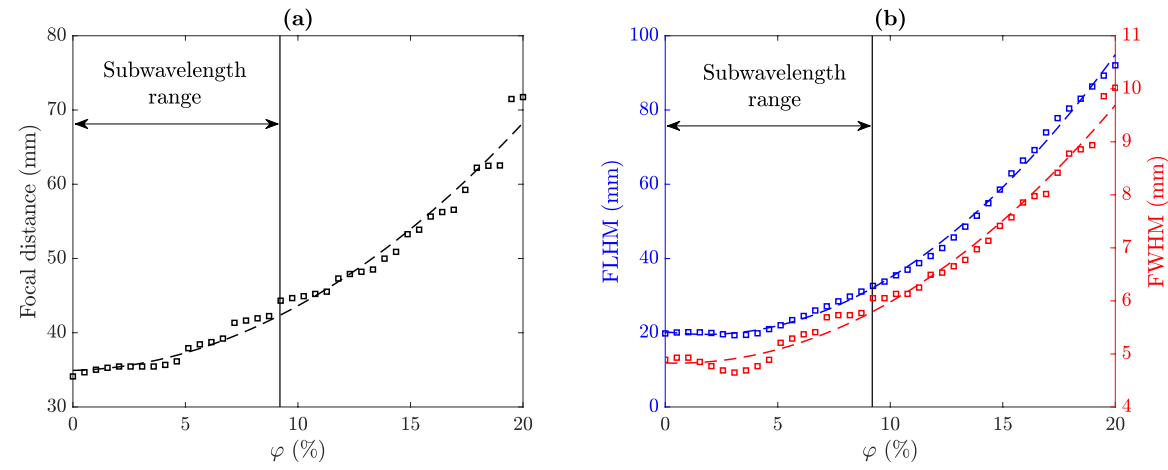

Figure 10.2: Simulated acoustic jet parameters vs. $\varphi$ : (a) focal distance, (b) FLHM (blue) and FWHM (red).

Therefore, the simulation results depicted in Figure 10.2 can be used to obtain the required volume fraction in order to achieve a specific focal distance and resolution. With this information, an automated mechanism could be implemented to adjust the volume fraction of the mixture as shown in Figure 10.1(a).
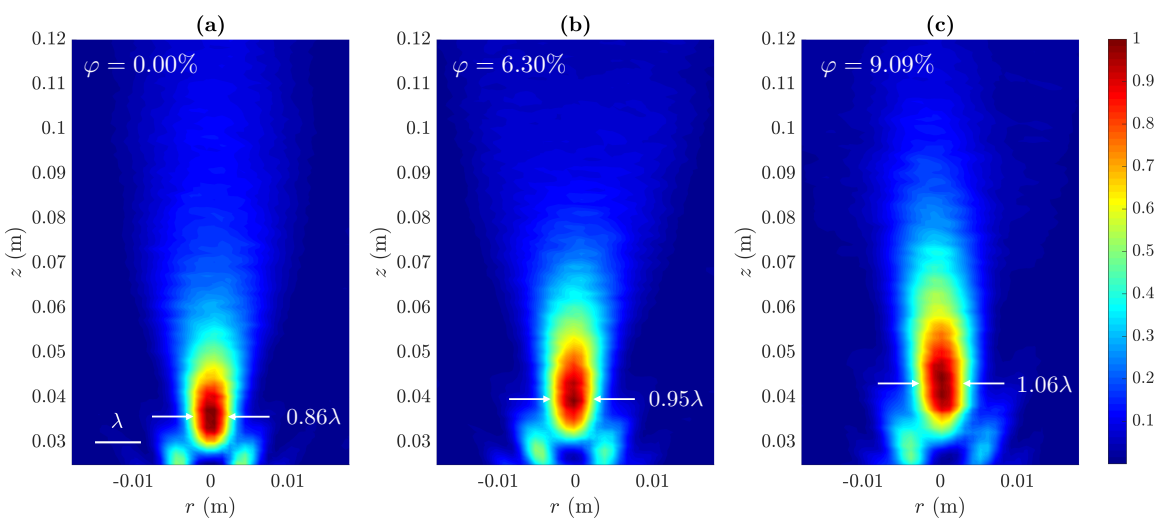

Figure 10.3: Measured normalized intensity maps: (a) $\varphi=0.00 \%$, (b) $\varphi=6.30 \%$ and (c) $\varphi=9.09 \%$.

Experimental results have been carried out in order to validate the proposed method. Figure 10.3 depicts the measured intensity maps for three different vol- 
ume fractions. Figure 10.3(a) corresponds to pure ethanol $(\varphi=0.00 \%)$, while 10.3 (b) and 10.3 (c) correspond to $\varphi=6.30 \%$ and $\varphi=9.09 \%$, respectively. Sound speed and density values for these volume fractions have been obtained from the polynomial fits shown in Figure 10.1. Each map is normalized to its maximum intensity value. As can be observed, there is a focal displacement in the acoustic jet, and the focal distance increases with the $\varphi$ parameter. Moreover, the focal area spreads out in both longitudinal and lateral directions, as expected from the simulation results shown in Figure 10.2.

Figure 10.4 depicts the focusing profiles for the three volume fractions that have been previously considered. Figure 10.4(a) shows the three computed focusing profiles for comparison purposes, while Figure 10.4(b) shows three separated plots with the experimental measurements (solid lines) and the simulations results (dashed lines) at each volume fraction. As can be observed from Figure 10.4, the pure Ethanol lens with $\varphi=0 \%$ provides the shortest focal distance with the highest longitudinal resolution, while the ethanol-water mixture with $\varphi=9.09 \%$ achieves the longest focal distance with the lowest resolution. The $\varphi=6.30 \%$ configuration provides intermediate values for both focal distance and longitudinal resolution.
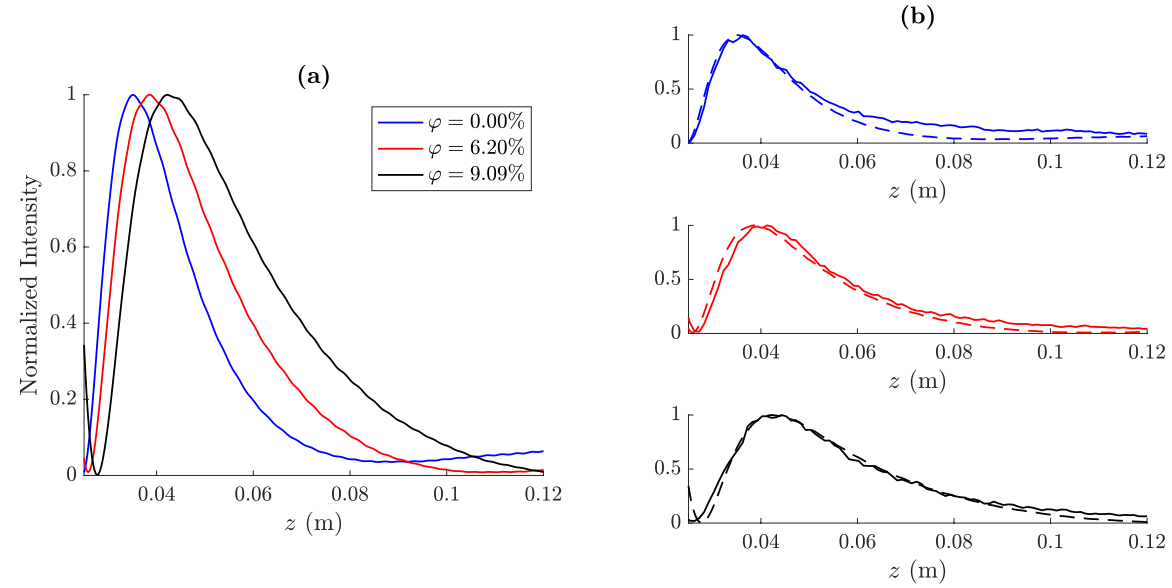

Figure 10.4: Focusing profiles at $r=0$ : (a) simulation results and (b) experimental measurements (solid lines) compared to their simulated counterparts (dashed lines).

Figure 10.5 depicts the transversal intensity cuts at $z=F$ for the three volume fractions that have been previously considered. Figure 10.5(a) shows the three computed transversal cuts for comparison purposes, while Figure 10.5(b) shows three separated plots with the experimental measurements (solid lines) and 
the simulations results (dashed lines) at each volume fraction. As can be observed from Figure 10.5, the pure ethanol lens $(\varphi=0 \%)$ achieves the highest lateral resolution providing the narrowest focal spot with a measured FWHM of $5.1 \mathrm{~mm}$, which corresponds to $0.856 \lambda$. The $\varphi=6.30 \%$ lens provides a measured lateral resolution of $5.638 \mathrm{~mm}(0.946 \lambda)$. Finally, the $\varphi=9.09 \%$ configuration shows the lowest resolution, with a FWHM of $6.293 \mathrm{~mm}(1.056 \lambda)$. These experimental results agree with the numerical simulations, and demonstrate that spherical lenses filled with ethanol-water mixtures can provide subwavelength resolution for a water volume ratio lower than $9 \%$, achieving a continuously tunable focal distance ranging from 35 to $43 \mathrm{~mm}$.
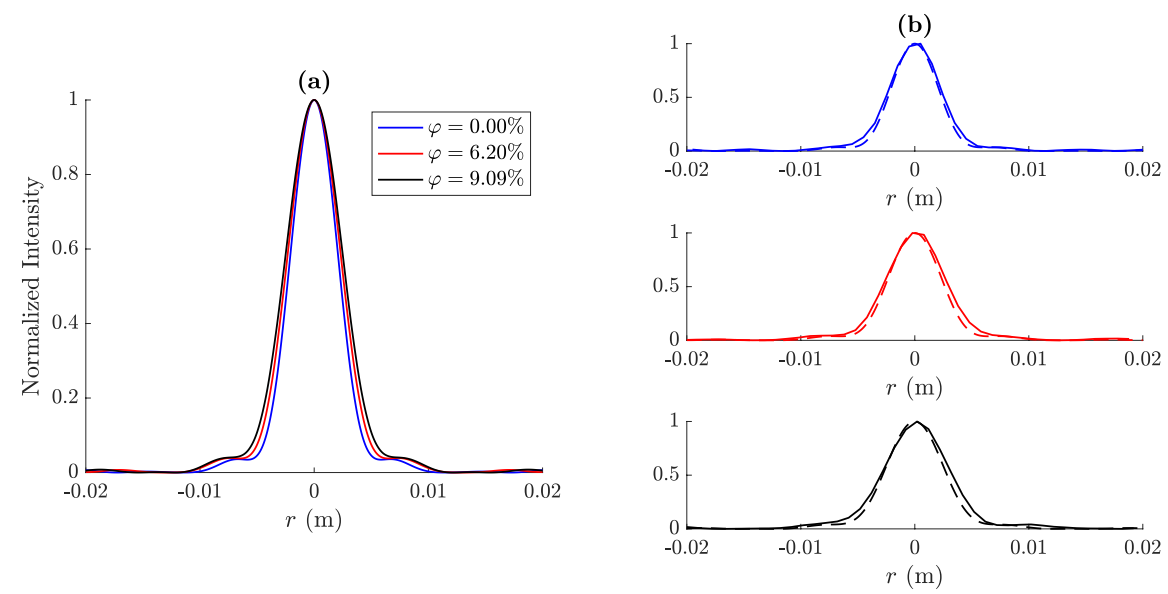

Figure 10.5: Transversal intensity cuts at $z=F$ : (a) simulation results and (b) experimental measurements (solid lines) compared to their simulated counterparts (dashed lines).

Table 10.1 shows the acoustic jet parameter simulation and experimental values for the three volume fractions previously considered. As can be observed from Figure 10.5 and Table 10.1, FEM results are in good agreement with experimental measurements. The lowest focal distance is achieved when the spherical lens is filled with pure ethanol $(\varphi=0 \%)$, and corresponds to a measured value of $F=35.25 \mathrm{~mm}$ or $F=5.91 \lambda$. The focal distance is shifted to 40.25 and $44.25 \mathrm{~mm}$, when the volume fraction is modified to $\varphi=6.30 \%$ and $\varphi=9.09 \%$, respectively. Lateral and longitudinal resolutions also exhibit similar tendencies with $\varphi$, increasing their value quadratically as the volume fraction augments and showing a linear dependence with the focal distance. Lateral resolution is below the wavelenght for both $\varphi=0 \%$ and $\varphi=6.30 \%$ achieving FWHM values of $0.86 \lambda$ and $0.95 \lambda$, respectively. These results demonstrate that the dynamic control of 
the liquid mixture sound speed allows a continuous shift of the focal length, while the lateral resolution can be kept below the wavelength for a certain focal length range.

\begin{tabular}{llcccccc}
\hline$\varphi(\%)$ & & $F(\mathrm{~mm})$ & $F(\lambda)$ & FLHM $(\mathrm{mm})$ & FLHM $(\lambda)$ & FWHM $(\mathrm{mm})$ & FWHM $(\lambda)$ \\
\hline \hline \multirow{2}{*}{0.00} & EXP & 35.25 & 5.91 & 20.61 & 3.46 & 5.10 & 0.86 \\
& FEM & 34.59 & 5.80 & 20.03 & 3.36 & 4.91 & 0.82 \\
\hline \multirow{2}{*}{6.30} & EXP & 40.25 & 6.75 & 26.03 & 4.37 & 5.64 & 0.95 \\
& FEM & 38.83 & 6.52 & 24.91 & 4.18 & 5.39 & 0.90 \\
\hline \multirow{2}{*}{9.09} & EXP & 44.25 & 7.42 & 31.45 & 5.28 & 6.29 & 1.06 \\
& FEM & 42.80 & 7.18 & 32.00 & 5.37 & 5.95 & 1.00 \\
\hline
\end{tabular}

Table 10.1: Measured (EXP) and simulated (FEM) acoustic jet parameters for different $\varphi$ values.

\subsection{Discussion}

Spherical lenses filled with liquid mixtures provide a continuous and dynamic control mechanism to adjust the lens focal distance, while maintaining a good lateral resolution. By shifting the volume ratio between both liquids, all acoustic jet parameters can be accurately tuned. A configurable spherical lens filled with ethanol and water has been experimentally characterized, measuring its acoustic intensity map for three different volume fractions. Simulation and experimental results show good agreement, demonstrating the performance of these lenses, which present a significance advantage over conventional single liquid spherical lenses, and are very appealing for many acoustic applications, such as acoustic microscopy. The lateral resolution is kept below the wavelength for a certain volume fraction range between $0 \%$ and $9 \%$. This lateral resolution could be further increased, going beyond the diffraction limit, by selecting two different miscible inner liquids, such as ethyl iodide and ethanol, with the requirement that one of these liquids (ethyl iodide) presents a higher sound speed contrast with the host medium. Numerical simulations show that a pure ethyl iodide lens can achieve a lateral resolution of $0.42 \lambda$, which is beyond the diffraction limit. Thus, this work opens new possibilities to implement higher resolution compact acoustic systems with continuous tunability of the main focusing parameters of the jet. 


\subsection{Methods}

\subsubsection{Numerical Model}

The acoustic pressure generated by the spherical lens has been numerically calculated using a Finite Element Method (FEM) model and the commercial software COMSOL Multiphysics. The numerical model is implemented as a $2 \mathrm{D}$ axisymmetric problem, because both transducer and spherical lens present rotation symmetry. The spherical container is made of ABS plastic with thickness $t_{h}=0.25 \mathrm{~mm}$ and diameter $D=40 \mathrm{~mm}$. The directional transducer has been implemented as a pressure condition at a distance $d=340 \mathrm{~mm}$ from the ABS sphere with $a=15$ $\mathrm{mm}$ being half the active radius of the transducer. The working frequency is fixed to $f_{0}=250 \mathrm{kHz}$, which provides a diameter to wavelength ratio of $D / \lambda=6.7$. A radiation condition is established at the boundaries in order to emulate the Sommerfeld radiation condition and avoid reflections. In order to consider the elastic properties of the solid ABS shell, the FEM model implements the acousticstructure multiphysics interface. ABS has been modelled as a linear elastic material characterized by its Young's modulus $\left(E_{s}\right)$, Poisson's ratio $\left(\nu_{s}\right)$ and density $\left(\rho_{s}\right)$. Figure 10.6 depicts a schematic diagram of the FEM model and Table 10.2 shows the properties of the different materials used in the simulation.

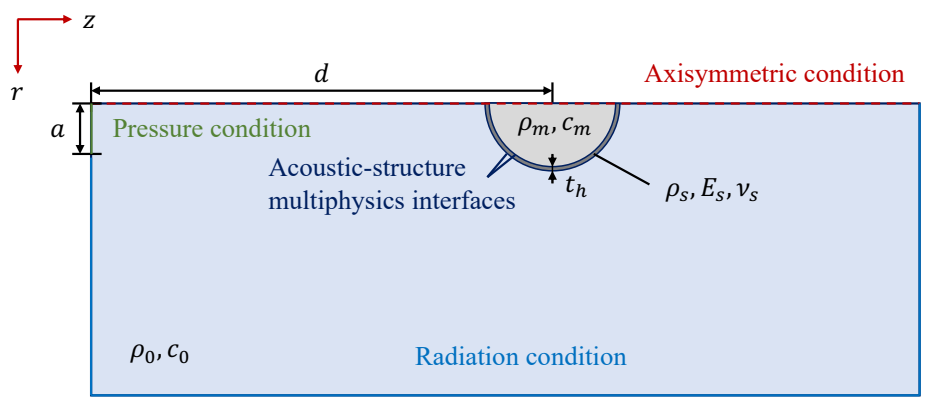

Figure 10.6: Schematic of the FEM model geometry and boundary conditions.

\begin{tabular}{ll}
\hline Material & Properties \\
\hline \hline Water & $\rho_{0}=1000 \mathrm{~kg} / \mathrm{m}^{3}, c_{0}=1490 \mathrm{~m} / \mathrm{s}$ \\
Ethanol & $\rho_{m}=793 \mathrm{~kg} / \mathrm{m}^{3}, c_{m}=1174 \mathrm{~m} / \mathrm{s}$ \\
ABS & $\rho_{s}=1050 \mathrm{~kg} / \mathrm{m}^{3}, E_{s}=3 \mathrm{GPa}, \nu_{s}=0.35$ \\
\hline
\end{tabular}

Table 10.2: Properties of the spherical lens materials. 


\subsubsection{Experimental Set-up}

Experimental measurements have been carried out in order to validate the numerical results. The experimental set-up consists of a 3D automated positioning system immersed in a $1 \times 0.5 \times 0.5 \mathrm{~m}^{3}$ tank filled with distilled water. A needle hydrophone with a diameter of $1.5 \mathrm{~mm}$ and $-4 \mathrm{~dB}$ bandwidth ranging from $200 \mathrm{kHz}$ to $25 \mathrm{MHz}$ is employed as receiver. The hydrophone is fixed to a programmable robotic arm, which can move in any of the three spatial directions with a resolution of $1 \times 1 \times 1 \mathrm{~mm}^{3}$. An Imasonic directional transducer with $30 \mathrm{~mm}$ of active diameter and a working frequency of $250 \mathrm{kHz}$ is used as emitter. Figure 10.7 shows a picture of the experimental set-up. The spherical lens is attached to a filament to keep it in place.

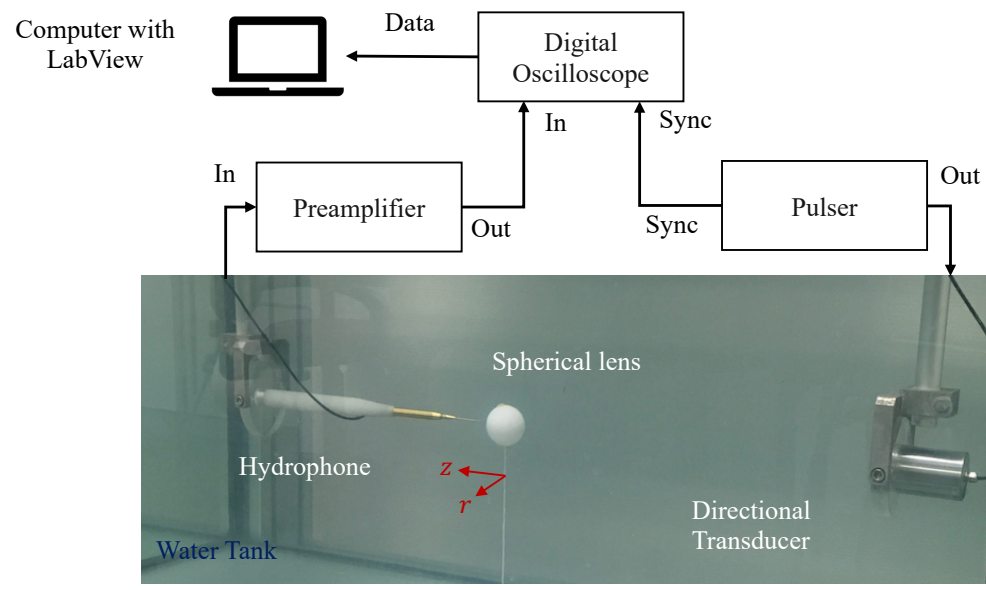

Figure 10.7: Experimental set-up.

\section{References}

[1] Y. F. Lu, L. Zhang, W. D. Song, Y. W. Zheng, and B. S. Luk'yanchuk, "Laser writing of a subwavelength structure on silicon (100) surfaces with particle-enhanced optical irradiation," Journal of Experimental and Theoretical Physics Letters, vol. 72, pp. 457-459, nov 2000.

[2] Z. Chen, A. Taflove, and V. Backman, "Photonic nanojet enhancement of backscattering of light by nanoparticles: a potential novel visible-light ultramicroscopy technique," Optics Express, vol. 12, no. 7, p. 1214, 2004. 
[3] A. Heifetz, S.-C. Kong, A. V. Sahakian, A. Taflove, and V. Backman, "Photonic Nanojets," Journal of Computational and Theoretical Nanoscience, vol. 6, pp. 1979-1992, sep 2009.

[4] D. C. Thomas, K. L. Gee, and R. S. Turley, "A balloon lens: Acoustic scattering from a penetrable sphere," American Journal of Physics, vol. 77, pp. 197203, mar 2009.

[5] M. A. Parrales Borrero, M. Pérez-Saborid, and J. M. Fernández García, "Acoustic scattering from a spherical lens irradiated by a finite transducer: Focusing effect and refraction," American Journal of Physics, vol. 79, pp. 401-408, apr 2011.

[6] O. V. Minin and I. V. Minin, "Acoustojet: acoustic analogue of photonic jet phenomenon based on penetrable 3D particle," Optical and Quantum Electronics, vol. 49, p. 54, feb 2017.

[7] J. H. Lopes, M. A. B. Andrade, J. P. Leão-Neto, J. C. Adamowski, I. V. Minin, and G. T. Silva, "Focusing Acoustic Beams with a Ball-Shaped Lens beyond the Diffraction Limit," Physical Review Applied, vol. 8, p. 024013, aug 2017.

[8] D. Veira Canle, T. Kekkonen, J. Mäkinen, T. Puranen, H. J. Nieminen, A. Kuronen, S. Franssila, T. Kotiaho, A. Salmi, and E. Hæggström, "Practical realization of a sub- $\lambda / 2$ acoustic jet," Scientific Reports, vol. 9, p. 5189, dec 2019.

[9] J. Chen, J. Xiao, D. Lisevych, A. Shakouri, and Z. Fan, "Deep-subwavelength control of acoustic waves in an ultra-compact metasurface lens," Nature Communications, vol. 9, p. 4920, dec 2018.

[10] B. Assouar, B. Liang, Y. Wu, Y. Li, J. C. Cheng, and Y. Jing, "Acoustic metasurfaces," Nature Reviews Materials, vol. 3, pp. 460-472, dec 2018.

[11] R. Al Jahdali and Y. Wu, "High transmission acoustic focusing by impedancematched acoustic meta-surfaces," Applied Physics Letters, vol. 108, p. 031902, jan 2016.

[12] S. Jiménez-Gambín, N. Jiménez, J. M. Benlloch, and F. Camarena, "Holograms to focus arbitrary ultrasonic fields through the skull," feb 2019.

[13] J. Hyun, Y. T. Kim, I. Doh, B. Ahn, K. Baik, and S.-H. Kim, "Realization of an ultrathin acoustic lens for subwavelength focusing in the megasonic range," Scientific Reports, vol. 8, p. 9131, dec 2018. 
[14] J. Chen, J. Rao, D. Lisevych, and Z. Fan, "Broadband ultrasonic focusing in water with an ultra-compact metasurface lens," Applied Physics Letters, vol. 114, p. 104101, mar 2019.

[15] J. Chen, Z. Sun, and Z. Fan, "Groove-structured meta-surface for patterned sub-diffraction sound focusing," Applied Physics Letters, vol. 114, p. 254102, jun 2019.

[16] H. Oku, K. Hashimoto, and M. Ishikawa, "Variable-focus lens with 1-kHz bandwidth," Optics Express, vol. 12, no. 10, p. 2138, 2004.

[17] M. Honma, T. Nose, S. Yanase, R. Yamaguchi, and S. Sato, "Liquid-crystal variable-focus lenses with a spatially-distributed tilt angles," Optics Express, vol. 17, p. 10998, jun 2009.

[18] C. B. Gorman, H. A. Biebuyck, and G. M. Whitesides, "Control of the Shape of Liquid Lenses on a Modified Gold Surface Using an Applied Electrical Potential across a Self-Assembled Monolayer," Langmuir, vol. 11, pp. 22422246, jun 1995.

[19] B. Berge and J. Peseux, "Variable focal lens controlled by an external voltage: An application of electrowetting," The European Physical Journal E, vol. 3, pp. 159-163, oct 2000.

[20] C. A. López and A. H. Hirsa, "Fast focusing using a pinned-contact oscillating liquid lens," Nature Photonics, vol. 2, pp. 610-613, oct 2008. 
Chapter 10. Tunable Subwavelength Ultrasound Focusing in Mesoscale Spherical Lenses using Liquid Mixtures 


\section{Chapter 11}

\section{Conclusions}

The aim of this thesis, as stated in Chapter 1, was to further develop the application of FZPs in the ultrasound domain and to investigate spherical liquid-filled lenses to generate tunable ultrasonic jets, expanding the use of this kind of acoustic lenses in FUS applications.

\subsection{Summary}

First of all, Chapter 1 presented a brief introduction to the main applications of FUS in both industrial and biomedical fields, and a discussion of the main methods employed to achieve acoustic focusing. Although phased-arrays are a very flexible approach to generate reconfigurable focusing patterns, they can be very expensive and bulky, which can be limiting in certain FUS applications. In this sense, acoustic metasurfaces and 3D printed holographic lenses appeared as a disruptive technique to design cheap and easy to manufacture acoustic lenses, capable of achieving complex pressure fields. However, metasurfaces and holographic lenses can be difficult to design, and often require performing complex time-consuming $3 \mathrm{D}$ wave propagation simulations and using optimization algorithms. Therefore, in some scenarios, simpler structures are preferred over more complex approaches. In this sense, FZPs are a very appealing choice, as they provide good focusing performance while keeping an easy design process and planar geometry. Moreover, spherical lenses capable of generating ultrasonic jets are emerging as one of the most appealing options to provide high-resolution beams for near-field FUS applications. Chapter 2 described the main theoretical equations and numerical 
methods used throughout this thesis to calculate the pressure fields generated by FZPs and spherical liquid-filled lenses. The Rayleigh-Sommerfeld technique is used to calculate the pressure field generated by diffraction at FZP lenses, while the ASA method is employed for propagating $2 \mathrm{D}$ pressure maps. For more complex simulations with multiphysics couplings, such as those required to analyze ultrasonic jets, FEM simulations are performed using COMSOL Multiphysics. Then, Chapters 3 to 6 extended the use of FZPs, adapting their design to the ultrasound domain and presenting a new beam modulation technique to control the ultrasound focus. These Chapters are related to objectives 1 and 2 of this thesis, established in section 1.3. Then, Chapters 7 and 8 demonstrated for the first time the application of binary sequences to modify the focusing profiles of FZPs in the ultrasound domain, which is related to objective 3 of this thesis. On the other hand, Chapters 9 and 10 presented a new design of spherical liquid-filled lenses capable of controlling the main focal properties of ultrasonic jets, demonstrating their use in near-field ultrasound focusing scenarios, which is related to objective 4 of this thesis.

Chapter 3 introduced a novel design technique to compensate the distortion introduced by ultrasound piston transducers, widely employed in ultrasound applications. In the far field, the pressure field generated by a piston can be described as a point source that generates spherical waves with a specific directivity pattern. This directivity pattern presents a main lobe and several secondary lobes. These secondary lobes introduce a $\pi$-phase shift to the generated pressure wavefront. If this $\pi$-phase shift is not compensated, the focusing profile of the lens is severely distorted, as the outer Fresnel regions of the lens illuminated by the secondary lobes introduce a destructive interference at the main focus of the FZP. In this sense, we introduced a new design technique, based on phase correction rings (PCRs). A PCR consists of skipping a Fresnel radius at the region where lens is illuminated by the secondary directivity lobe instead of the main lobe. This skip introduces an additional $\pi$-phase shift that compensates that introduced by the secondary lobe of the directivity pattern, therefore avoiding the destructive interference at the FZP focus. Experimental results were presented, showing a $150 \%$ increase at the focal intensity when a PCR is employed.

Chapter 4 presented a design technique capable of achieving a bifocal focusing profile using a combination of two conventional FZPs. The method is based on designing the inner Fresnel regions of the lens to achieve a focal distance $F_{1}$, while the outer regions of the lens are designed for a distance $F_{2}>F_{1}$. The combination of $F_{1}, F_{2}$, and the design frequency has to be optimized such that the phase of the focusing profile of the inner and outer regions do not interfere destructively neither at $F_{1}$ nor at $F_{2}$. Experimental and numerical results were presented, showing very good agreement, demonstrating the use of this design method and expanding the capabilities of FZPs in FUS scenarios. 
Chapter 5 developed a theoretical analysis of the transient response of FZP lenses. Due to their planar geometry, FZPs present a transient state, as the time of flight from the lens to the focus is longer for the outer regions (longer propagation paths) than for the inner regions (shorter propagation paths). Therefore, the lens only achieves a steady response after a transient state, when all the regions contribute to the main focus simultaneously. If the pulse duration is shorter than the transient state duration, simulations showed that the lens would exhibit degraded focusing performance in terms of focal intensity and axial and lateral resolutions. Thus, the transient state should be considered to achieve optimum focusing parameters when using short ultrasound pulses.

Chapter 6 introduced a novel technique capable of modulating the ultrasound beam of a conventional FZP in both space and time. The technique is based on the linear focal distance dependence on the operating frequency, meaning that, for a given FZP, the focal distance is shifted closer to the lens when the operating frequency is reduced from its theoretical design frequency, and shifted away from the lens when the operating frequency is increased. Therefore, it is possible to create a custom waveform of contiguous rectangular pulses with frequencies and amplitudes calculated to provide focal spots with the desired acoustic intensities and focal distances, at specific times and in the designed sequence, therefore modulating the response of the lens in space and time. Experimental results of a two foci and a three foci configuration were presented, showing excellent agreement with numerical results and demonstrating the feasibility of the modulation technique.

Chapter 7 analyzed the use of Cantor fractal binary sequences applied to a conventional FZP to modulate its focusing profile. These binary sequences are used to decide whether a Fresnel region is activated or not. It is shown that, if the governing binary sequence is a fractal Cantor set, the lens presents a multifocal focusing profile with interesting self-similarity properties. Two Cantor lenses were manufactured, one with a central transparent region and other with a central pressure blocking area. Due to the directional behaviour of the piston transducer, the Cantor lens with the transparent central region achieved a higher focal intensity level compared to the central pressure blocking design. It was numerically and experimentally demonstrated, for the first time in the ultrasound domain, that Cantor binary sequences are an excellent approach to extend the capabilities of conventional FZPs.

Chapter 8 showed a new acoustic lens design based on applying a $\mathrm{M}$ bonacci binary sequence to the different regions of a FZP lens. This kind of $M$-bonacci lenses provides bifocal focusing profiles with two equal intensity foci. The ratio between the two focal distances is fixed, and it depends on the type of $\mathrm{M}$ bonacci sequence. When $m=2$ (which corresponds to a conventional Fibonacci 
sequence), the focal ratio is 1.618 , which is directly related to the golden mean, while when $m=3$ and $m=4$ the ratios are 1.192 and 1.078 , respectively. In addition, a new distortion parameter is introduced, $\gamma$, which allows to shift the focal distances of M-bonacci lenses from their constrained theoretical distances. Experimental results were presented, demonstrating the feasibility of the approach for ultrasound focusing applications.

Chapter 9 introduced a new acoustic lens design based on a liquid-filled spherical container that can generate subwavelength ultrasonic jets. Depending on the inner liquid, the main focusing parameters of the ultrasonic jet can be controlled, such as the focal distance and the axial and lateral resolutions. Experimental results for an Ethanol-filled ABS container with a diameter of $6.67 \lambda$ were presented, achieving a lateral resolution of $0.85 \lambda$ and demonstrating the potential of this kind of devices for near-field ultrasound imaging applications. Numerical simulations also showed that liquids with a higher speed of sound contrast with water are capable of generating ultrasonic jets with lateral resolutions beyond the diffraction limit.

Finally, Chapter 10 developed a novel design technique based on liquid mixtures capable of continuously tuning the focusing parameters of the ultrasonic jet. The spherical lens consists of a thin ABS container, filled with a mixture of ethanol and deionized water. Depending on the mixing ratio, the acoustic parameters of the mixture can be accurately tuned (i.e. its speed of sound and density), and therefore the main properties of the ultrasonic jet can be controlled. Numerical and experimental results were shown, achieving a focal distance range between 34.6 and $42.8 \mathrm{~mm}$ while keeping lateral subwavelength resolution.

\subsection{Main Conclusions}

The main conclusions of this work can be summarized in the following main points:

- The influence of the transducer has to be considered during the design process of acoustic lenses. In particular, when using FZPs, the directivity pattern of the transducer produces severe distortion on the focusing profile of the lens, resulting in reduction of the focal intensity and resolution. To compensate this distortion, PCRs should be employed to compensate the phase shift introduced by of the secondary lobes of the directivity pattern. This is related to objective 1 of this thesis.

- It has been experimentally demonstrated that multifocal focusing profiles can be achieved using monofocal FZP lenses, either by combining two con- 
ventional FZPs or by applying binary sequences that control the activation of the different Fresnel regions of the lens. When combining two FZPs with different focal distances to generate a bifocal lens, the axial phase profile of each FZP has to be considered in order to achieve an optimum bifocal profile. Using binary sequences provides a new degree of flexibility to traditional FZP lenses. In particular, it has been shown that Cantor binary sequences provide interesting multifocal profiles, and $\mathrm{M}$-bonacci sequences are able to generate bifocal profiles in the ultrasound domain. This is related to objectives 2 and 3 of this thesis.

- The linear dependence of the focal distance on the operating frequency can be used to increase the versatility of FZP lenses. A new spatio-temporal beam modulation method that takes advantage of this linear dependence has been presented, demonstrating that the position of the ultrasonic focus generated by a conventional FZP lens can be controlled in both space and time. The intensity level of each focus can be individually tuned, as well as the duration of the pulse and its order in the multifocal sequence. This further expands the potential applications of FZPs in both NDT and biomedical scenarios. This is related to objective 3 of this thesis.

- Liquid-filled spherical lenses can be used as a very flexible tool to generate ultrasonic jets. Changing the inner liquid of the spherical container modifies the acoustic properties of the lens, and therefore allows to control the main properties of the jet. In addition, if the inner liquid is a mixture of two substances, the acoustic parameters of the jet can be continuously tuned by changing the mixing ratio between both liquids. This is related to objective 4 of this thesis.

Future work could include using multilevel 3D printed FZP designs instead of conventional Soret FZPs made of brass or other acoustically opaque materials, which would increase the power efficiency of this kind of devices while reducing their manufacturing cost and time. The influence of other types of binary sequences on the focusing profiles of FZP lenses could also be explored, as applying binary sequences to the different regions of an FZP has proven to be a very powerful tool to modify the focal properties of the lens. In addition, designing 3D printed acoustic holograms with focal parameters that present linear dependence on the operating frequency could provide a new degree of flexibility to this type of lenses, as spatio-temporal beam modulation techniques, such as that presented in this work, could be applied to dynamically modulate the ultrasonic beam.

In summary, this thesis devised new design methods and applications of FZP lenses in the ultrasound domain, compensating the distortion introduced by ultrasound transducers, extending their focusing capabilities with new spatio- 
temporal beam modulation techniques, and applying binary sequences to shape their focusing profile. In addition, spherical liquid-filled lenses were presented as a fast, simple, and tunable method to generate ultrasonic jets, which are becoming a very appealing tool for near-field imaging applications. These results, I hope, can pave the way for simpler yet versatile and powerful ultrasound focusing systems. 


\section{Funding Acknowledgement}

Finally, this work would not have been possible without the following funding sources:

- PAID-01-18 personal FPI grant from Universitat Politècnica de València.

- Spanish government MINECO TEC2015-70939-R project.

- Spanish government MICINN RTI2018-100792-B-I00 project.

- Generalitat Valenciana AICO/2020/139 project. 